Universidad deValladolid

PROGRAMA DE DOCTORADO EN FÍSICA

TESIS DOCTORAL:

\title{
ELECTRONIC TONGUE TECHNOLOGY APPLIED TO THE ANALYSIS OF GRAPES AND WINES
}

Presentada por Celia García Hernández para optar al grado de

Doctor/a por la Universidad de Valladolid

Dirigida por:

Prof. Dra. María Luz Rodríguez Méndez

Prof. Dra. Ana Cristina García Cabezón 



\section{CQSDUVa \\ Escuela de Doctorado Universidad de Valladolid}

Universidad deValladolid

\section{AUTORIZACIÓN DEL DIRECTOR/A DE TESIS}

Impreso 1T

(Art. 7.2 de la Normativa para la presentación y defensa de la Tesis Doctoral en la UVa)

Da María Luz Rodríguez Méndez, con D.N.I. 50305983-S, y Da Ana Cristina García Cabezón, con D.N.I. 16804269-D,

Profesoras del departamento de Química Física y Química Inorgánica y del departamento de Ciencia de los Materiales e Ingeniería Metalúrgica, respectivamente.

Centro Escuela de Ingenierías Industriales.

Dirección a efecto de notificaciones:

Escuela de Ingenierías Industriales

Universidad de Valladolid

Paseo del Cauce, 59

47011 Valladolid

Phone:+34983423540 / +34983423389

e-mail: mluz@eii.uva.es / crigar@eii.uva.es

como Directoras de la Tesis Doctoral titulada Electronic tongue technology applied to the analysis of grapes and wines.

realizada por Da Celia García Hernández.

alumna del Programa de Doctorado en Física

autorizan su presentación, considerando que cumple los requisitos exigidos para ello.

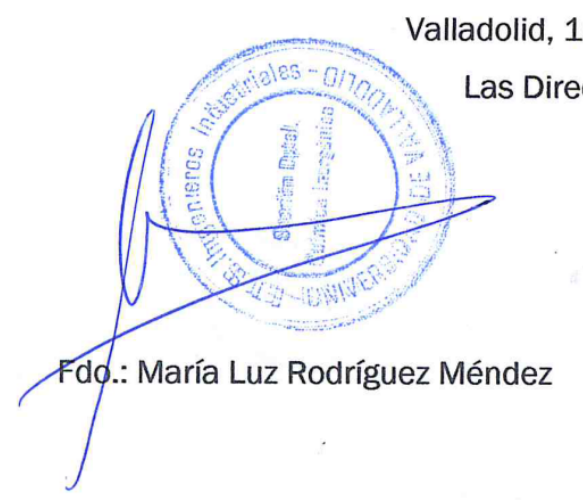

Las Directoras de la Tesis,

SR/SRA. PRESIDENTE/A DE LA COMISIÓN DE DOCTORADO 

The dissertation of Celia García Hernández was reviewed and approved* by the following External Evaluators:

Ph.D. Carlos José Leopoldo Constantino

Full professor

University of São Paulo State (UNESP), Brazil

Ph.D. Luis G. Dias

Assistant Professor

Polytechnic Institute of Bragança, Portugal

*Assessment reports are annexed to this dissertation. 



\section{Acknowledgements}

En primer lugar, mi agradecimiento más sincero a las Profesoras María Luz Rodríguez Méndez y Ana Cristina García Cabezón, por darme la oportunidad de formar parte de su equipo de investigación, por todo el esfuerzo y dedicación que han puesto en mí durante el desarrollo de esta Tesis Doctoral y por transmitirme tanto conocimiento.

Al Profesor Fernando Martín Pedrosa, por su ayuda en el tratamiento estadístico de los datos, su plena disposicion, su amabilidad y buen trato.

A mis compañeros y colegas de laboratorio, que han sido muchos durante estos años, por su compañía y amistad, que han hecho más agradable el trabajo. En especial a la Profesora Raquel Muñoz, por su siempre cariñoso trato, buenos consejos y ayuda.

En estos momentos también quiero tener un especial recuerdo al Profesor José Antonio de Saja, fallecido en 2017, por su ayuda y buen trato durante el inicio de mi carrera investigadora.

A las bodegas y laboratorios enológicos que han hecho posible el desarrollo de esta Tesis, agradezco mucho su disposición y buen trato, en especial a José Antonio Fernández Escudero, Germán Medrano y Enrique Barajas.

Al Profesor Adam Wanekaya, de la Universidad Estatal de Misuri, por acogerme en su laboratorio y enseñarme durante mi estancia de investigación en el extranjero.

A Jhon, por su apoyo, abundante paciencia y por no dejarme flaquear en los momentos más difíciles.

Y mi mayor agradecimiento es para mi familia, mis padres, hermanos y sobrinos. En especial a mis padres, por su gran ayuda y apoyo constante, por infundirme ánimo en los momentos más duros del camino. Muchas gracias por la dedicación y el amor que siempre habéis puesto en vuestros hijos. 

A mis padres,

por su amor,

por dármelo todo. 

Doctoral Thesis: Electronic tongue technology applied to the analysis of grapes and wines

\section{TABLE OF CONTENTS}

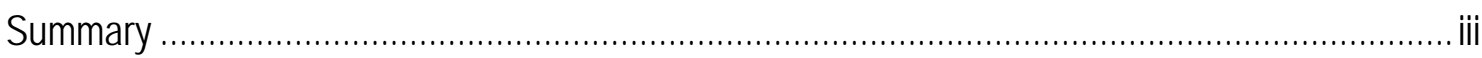

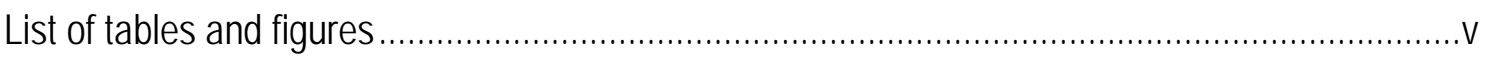

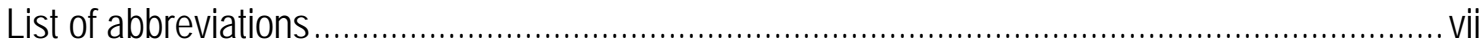

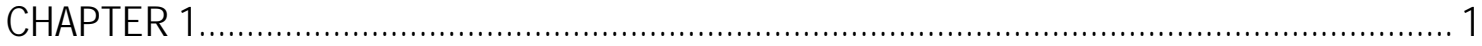

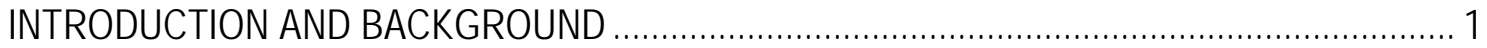

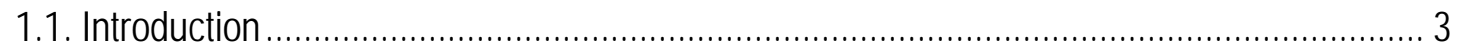

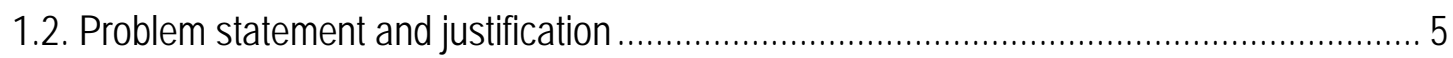

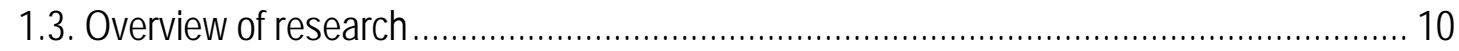

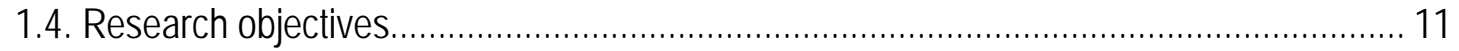

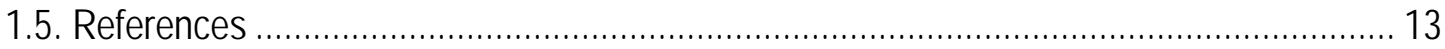

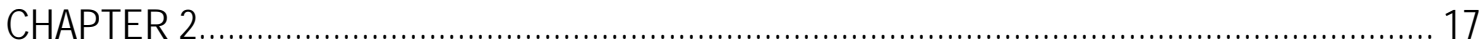

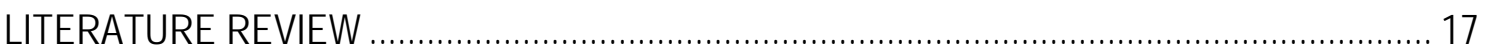

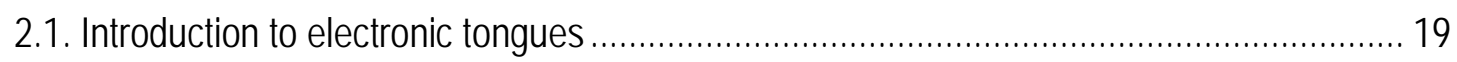

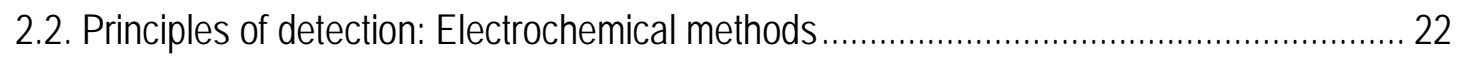

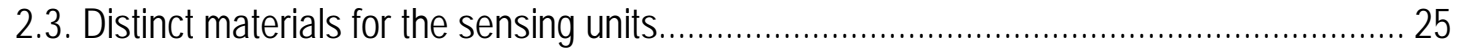

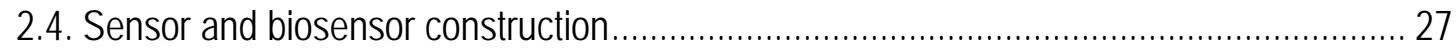

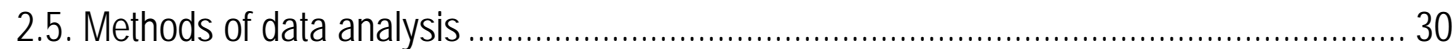

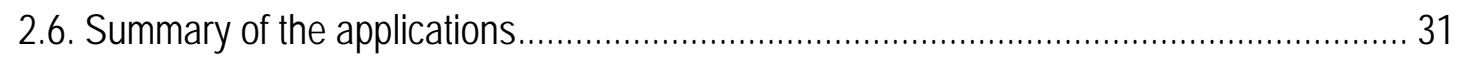

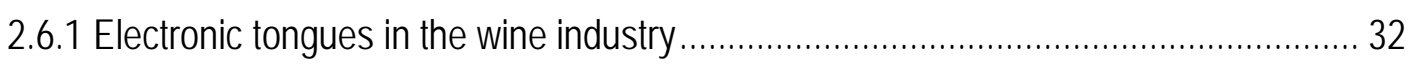

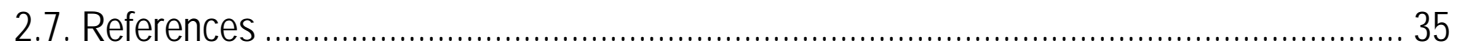

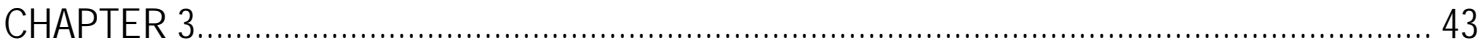

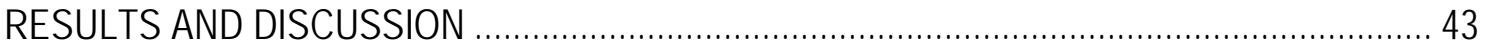

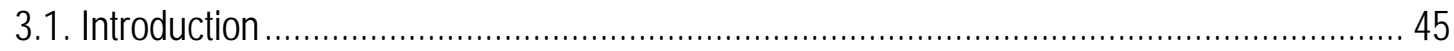

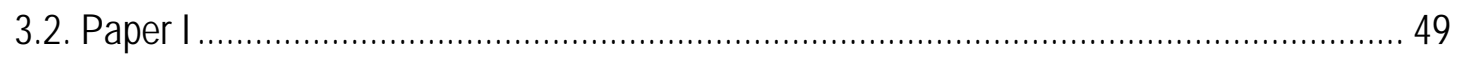

Multisensor systems based on phthalocyanines for monitoring the quality of grapes.............. 49

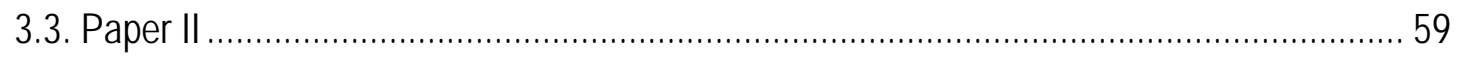

Monitoring the phenolic ripening of red grapes using a multisensor system based on metal-

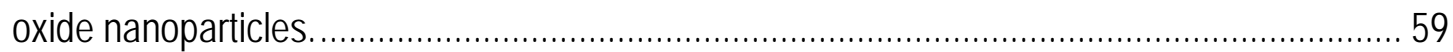

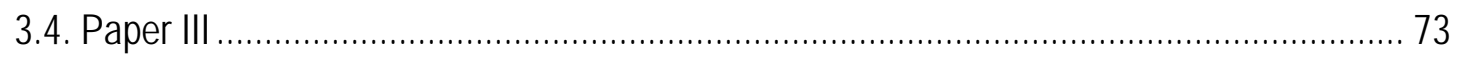

A different approach for the analysis of grapes: Using the skin as sensing element. ............. 73 
Doctoral Thesis: Electronic tongue technology applied to the analysis of grapes and wines

3.5. Paper IV

Electrochemical behavior of polypyrrol/AuNP composites deposited by different electrochemical methods: sensing properties towards catechol

3.6. Paper V

Analysis of musts and wines by means of a bio-electronic tongue based on tyrosinase and glucose oxidase using polypyrrole/gold nanoparticles as the electron mediator. 103

3.7. Paper VI

Layered composites of PEDOT/PSS/nanoparticles and PEDOT/PSS/phthalocyanines as electron mediators for sensors and biosensors

3.8. Paper VII

Impedimetric electronic tongue based on nanocomposites for the analysis of red wines. Improving the variable selection method

3.9. Paper VIII

An electrochemical quartz crystal microbalance multisensor system based on phthalocyanine nanostructured films: Discrimination of musts. 145

3.10. Paper IX 169

In situ synthesis, stabilization and activity of protein-modified gold nanoparticles for biological applications.

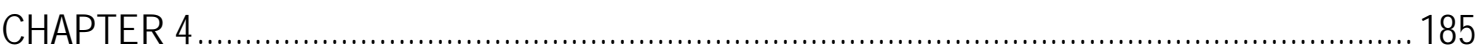

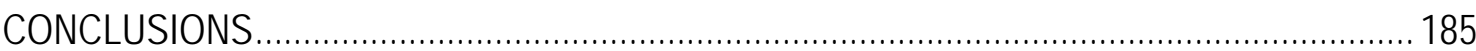

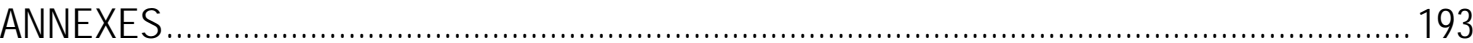

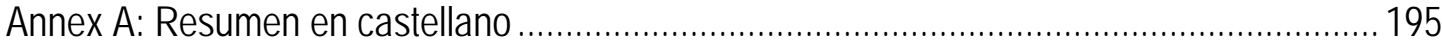

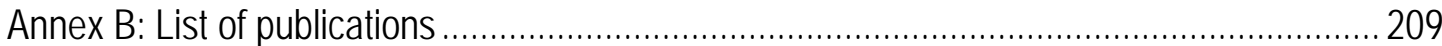

Annex C: International research stay attestation letter......................................................... 217

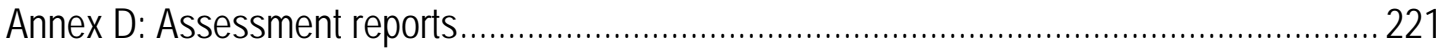


The Doctoral Thesis entitled "Electronic tongue technology applied to the analysis of grapes and wines" has been developed in the group of sensors UVaSens under the supervision of Prof. PhD. María Luz Rodríguez Méndez, from the Inorganic Chemistry and Physical Chemistry Department at the Engineers School, and Prof. PhD. Ana Cristina García Cabezón, from the Materials Science and Metallurgical Engineering Department at the Engineers School, both from the University of Valladolid (Spain). The author of this Thesis has carried out a research stay at the Missouri State University (Springfield, EE.UU.) under the supervision of Prof. PhD. Adam Wanekaya for 4 months to be able to obtain an international Ph.D. mention.

This dissertation contains research that studies the use of sensors and biosensors implemented as arrays of electrodes in electronic and bioelectronic tongues to analyze quality characteristics of wines and grapes of interest in the agrofood industry such as sugar and/or polyphenolic content. Different and novel electrocatalytic materials have been used and combined to construct the arrays of sensors and biosensors. The samples of wine and grape used in this study were provided by the wineries "Cooperativa de Cigales" and "Instituto Tecnológico Agrario de Castilla y León (ITACyL)", both located in the Castilla y León region (Valladolid, Spain). The chemical parameters of the samples were analyzed in the UVaSens laboratory, and also by ITACyL, following international regulations (OIV, The International Organisation of Vine and Wine).

The following provides an overview of the structure of this dissertation: Chapter 1 provides an introduction and background to this research. A literature review is presented in Chapter 2 including the state of the art, the applications of these devices in the food industry, the type of sensors used to develop electronic tongues, the electrochemical techniques applied, the electrocatalytic materials that help to improve the responses of the electronic tongues, the techniques used to fabricate nanostructured films to modify sensors, as well as the most common statistical tools implemented to analyze data obtained from electronic tongues. Chapter 3 looks at 9 research papers with the results obtained from the studies carried out and published in international journals with impact factor. An overview of the conclusions from the entire dissertation is presented in Chapter 4.

Finally, Annexes A, B, C and D contain a summary written in Spanish in accordance with the regulations of the University of Valladolid, the co-authored list of papers published during the Doctoral studies, the attestation letter of the international research stay carried out in the 
Chemistry Department of the Missouri State University (Springfield, USA) signed by the supervisor Prof. Wanekaya and the assessment reports provided by the External Evaluators. 


\section{Tables}

Table 1. Overview of classical chemical analysis and modern analytical instrument techniques

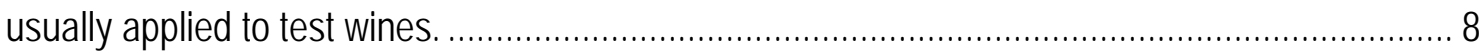

Table 2. Advantages of electronic tongues over other methodologies...................................... 10

Table 3. Examples of electrochemical electronic tongues applied to food analysis. .................... 24

\section{Figures}

Figure 1. Schematic presentation of the electronic tongue................................................... 20 

$a^{*}$

AAS

AFM

ANNS

ATR

AuNPs

$b^{*}$

B\%

bioET

BKG

$C^{*}$

$\mathrm{CA}$

CD

$\mathrm{Cl}$

CNT

$\mathrm{CP}$

CPS

CPE

$d A \%$

DLS

D.O.

DSA

EDP

EIS

EM

EQCM

$E T$, e-tongue

FIA

FTIR, FT-IR

FT-NIR

$h^{*}$

$\mathrm{H}$

GC

GC-MS

GC-MS/MS

GC-O

\section{Redness}

Atomic absorption spectroscopy

Atomic force microscopy

Artificial neural networks

Attenuated total reflection

Gold nanoparticles

Yellowness

Proportion of blue color

Bioelectronic tongue

Background

Chroma or saturation

Chronoamperometry

Color density

Color intensity

Carbon nanotube

Chronopotentiometry

Conducting polymers

Carbon paste electrode

Proportion of red color produced by flavylium cations

Dynamic light scattering

Denomination of Origin

1-decanesulfonic acid sodium salt

Electrodeposition

Electrochemical impedance spectroscopy

Electrocatalytic material

Electrochemical quartz crystal microbalance

Electronic tongue

Flow injection analysis

Fourier transform infrared spectroscopy

Fourier transform near-infrared spectroscopy

Hue angle

Hue

Gas chromatography

Gas chromatography combined with mass spectrometry

Gas chromatography combined with tandem mass spectrometry

Gas chromatography combined with olfactometry 


\begin{tabular}{|c|c|}
\hline GOx & Glucose oxidase enzyme \\
\hline HPLC & High-performance liquid comatography \\
\hline $\mathrm{HQ}$ & Hydroquinone \\
\hline IC & Ion chromatography \\
\hline ICP-MS & Inductively coupled plasma mass spectrometry \\
\hline IRMS & Isotope ratio mass spectrometry \\
\hline ISFETS & Ion-selective field effect transistors \\
\hline ITO & Indium tin oxide \\
\hline$L^{*}$ & Lightness \\
\hline Lac & Laccase \\
\hline LB & Langmuir-Blodgett \\
\hline LbL & Layer by Layer \\
\hline LC & Liquid chromatography \\
\hline LC-MS/MS & Liquid chromatography combined with tandem mass spectrometry \\
\hline LDA & Linear discriminant analysis \\
\hline $\mathrm{LnPC}_{2}$ & Lanthanides bis-phthalocyanine \\
\hline LOD, LD & Limit of detection \\
\hline LV & Latent variables \\
\hline Lys & Lysozyme \\
\hline MIP & Molecular imprinted polymer \\
\hline MOFs & Metal-organic frameworks \\
\hline MONPS & Metal oxide nanoparticle \\
\hline MPcs & Metallophthalocyanines \\
\hline MPs & Metalloporphyrins \\
\hline MS & Mass spectrometry \\
\hline MUA & 11-mercapto-undecanoic acid \\
\hline MWCNT & Multiwall carbon nanotube \\
\hline NIR & Near-infrared spectroscopy \\
\hline NMR & Nuclear magnetic resonance spectroscopy \\
\hline N-PLS & $\mathrm{N}$-way partial least squares \\
\hline PA & Polyacetylene \\
\hline $\mathrm{PAH}$ & Poly(allylamine hydrochloride) \\
\hline PANI & Polyaniline \\
\hline PARC & Pattern recognition \\
\hline PARAFAC & Parallel factor analysis \\
\hline Pc & Phthalocyanine \\
\hline PC & Principal component \\
\hline
\end{tabular}




\begin{tabular}{|c|c|}
\hline PCA & Principal component analysis \\
\hline PCR & Principal component regression \\
\hline PEDOT & Poly (3,4-ethylenedioxythiophene) \\
\hline PEI & Poly(ethyleneimine) \\
\hline pl & Isoelectric point \\
\hline Ppy, PPy & Polypyrrole \\
\hline PSS & Polystyrene sulfonate \\
\hline PLS & Partial least squares \\
\hline PLS-DA & Partial least squares discriminant analysis \\
\hline PT & Polythiophene \\
\hline PVC & Poly(vinyl chloride) \\
\hline QCM & Quartz crystal microbalance \\
\hline $\mathrm{R} \%$ & Proportion of red color \\
\hline $\mathrm{RI}$ & Refractive index \\
\hline RMSE & Root mean square error \\
\hline RSD & Relative standard deviation \\
\hline SAM & Self-assembled monolayer \\
\hline SEM & Scanning electron microscopy \\
\hline S-CPE & Skin carbon paste electrode \\
\hline SIMCA & Soft independent modeling of class analogy \\
\hline SIRA & Stable isotope ratio analysis \\
\hline SPE & Screen printed electrode \\
\hline SVM & Support vector machines \\
\hline SVMR & Support vector machines regression \\
\hline SS & Stainless steel \\
\hline TA & Total acidity \\
\hline TEM & Transmission electron microscopy \\
\hline TPI & Total polyphenol index, phenolic content \\
\hline Tyr & Tyrosinase \\
\hline UHPLC/Q-TOF-MS & $\begin{array}{l}\text { Ultra-high performance liquid chromatography-quadrupole time-of- } \\
\text { flight mass spectrometry }\end{array}$ \\
\hline UV-Vis & Ultraviolet and visible spectroscopy \\
\hline VOCs & Volatile organic compounds \\
\hline WE & Working electrode \\
\hline Y\% & Proportion of yellow color \\
\hline ZP & Zeta potential \\
\hline
\end{tabular}



Doctoral Thesis: Electronic tongue technology applied to the analysis of grapes and wines

\section{INTRODUCTION AND BACKGROUND}





\subsection{Introduction}

The concept of electronic tongues (ETs or e-tongues) or taste sensors has grown rapidly during recent years due to their large potential. They are based on electrochemical sensors combined with multivariate data analysis. The development of new methods of analysis to characterize food is of vital importance for improving the current quality control systems of food products. According to the IUPAC (International Union of Pure and Applied Chemistry), an electronic tongue is a multisensor system, which consists of a number of low selective sensors and uses advanced mathematical procedures for signal processing based on the pattern recognition (PARC) and/or multivariate analysis [artificial neural networks (ANNs), principal component analysis (PCA), etc.] [1]. Therefore, ETs are holistic systems that provide global and qualitative information about the sample instead of quantitative data about specific compounds. However, if the data matrix obtained by such multisensor systems is analyzed with adequate chemometric processing tools, descriptive or predictive information of particular parameters could be extracted $[2,3]$. There is a most recent term in the field of electronic tongues widely named bioelectronics tongue (bioET), which includes the use of one or several biosensors implemented in the ETs $[4,5]$.

This research examines how ETs and bioETs can be applied to study red grapes and wines in order to better predict the harvesting time of grapes and quality parameters of interest to produce high quality wines. Routine analytical tests from the harvesting of grapes to the final bottled end-product include a number of varying chromatographic techniques, as well as more traditional ones (spectroscopy, photometry, enzymatic assays, etc.). These analytical techniques will be combined to provide an in-depth study of the composition of wines leading to analyses which are very expensive, time consuming and demand highly qualified personnel. It is therefore necessary to develop new technologies that allow us to obtain information concerning the spectrum of compounds, including global information about the sample instead of information about specific components [6]. ETs can become an alternative or complement to the traditional methods of wine and grape analysis that could be used, not only in the analytical laboratories, but also in situ as a quality control tool for the farmers and wine growers in the vineyards.

In addition, nowadays, we are immersed in the fourth revolution of the manufacturing industry, also called Industry 4.0 (or smart factory, cloud-based manufacturing, factory of the future, smart manufacturing and digital manufacturing). This next big manufacturing revolution will demand benefits from joining multidisciplinary competences related to new sensing technologies, 
big data, cloud computing, artificial intelligence, adaptive robots, smart valves and autonomous smart control applications to bring maximum added value in future smart processes. Existing technologies exploited by measurement, control and automation systems are mainly based on measuring physical parameters. The next big improvement will be to deploy real-time chemical information from the measurement objects to an automation database and use it in automated control decisions. Spectroscopic technologies are well-established in off-line analysis in chemical laboratories, but it is obvious that future smart factories will gain huge benefits when qualitative and quantitative material information can be generated with affordable and reliable sensors. Realtime chemical sensors will open up a new era of process control in several industries. Industrial processes of food and beverages will benefit more and more in the future from next generation smart sensor platforms $[7,8]$.

On the other hand, there are not many places in the world as appropriate as Spain for studying high quality wines. Spain has a millenary tradition and history in the wine industry. Moreover, Spain has 69 wine Denominations of Origin, Rioja, Ribera del Duero and Rueda being some of the most relevant. Denominations of Origin are not solely an administrative status, but also the way used in Spain to recognize and protect a product with a high quality and unique characteristics, origin and tradition. According to the OIV Statistical Report 2018 [9], Spain was one of the largest producers of wine around the world, first in the ranking of planted surface area, third in production of wine excluding juice and musts (after Italy and France), fifth in grape production (after China, Italy, USA and France) and the largest exporter around the world in terms of volume (followed by Italy and France), third in terms of value. Additionally, Spain is not an importer of wine (neither in terms of volume nor value) as other important wine producer countries, are such as Italy or France.

However, besides being of great importance for the Spanish food industry, why has wine become so essential in the Mediterranean diet? This is due to the antioxidant properties of polyphenols which provide health benefits. It has been demonstrated that the consumption of antioxidants decreases the risk of cardiovascular diseases [10-12]. For this reason, international organisms recommend the daily ingestion of foods rich in antioxidants [13].

The primary goal of this research is to develop new electrochemical sensors to find appropriate electroactive and/or sensing materials able to interact with antioxidants and polyphenols contained in grape musts and wines to be implemented in electronic and bioelectronics tongues. Some candidates for this task will be conducting polymers, metal 
nanoparticles, metal oxide nanoparticles, phthalocyanines and/or enzymes. In this aspect, the use of nanotechnology can play an important role in manufacturing nanostructured sensors by using different surface modification techniques that will provide chemically modified electrodes. Also, further improvements in sensors responses when combining some of the mentioned materials will be discussed. Then, the sensors will be combined to form an array of electrodes constituting an ET and/or bioET that will be used to analyze real samples of wines and grape musts. Finally, statistical analyses will be carried out to discriminate between samples, to establish correlations between the data obtained with the ET/bioET and the chemical parameters obtained by traditional analysis and, finally, to predict quality parameters of interest by using the data obtained with the ET/bioET.

\subsection{Problem statement and justification}

Wine, one of the oldest alimentary products known for more than 8000 years, is an alcoholic beverage obtained through the fermentation of grapes. The origin of viticulture dates back to $6000-5000$ BC in Georgia and Iran. Winemaking culture still remains unchanged: modern wine is very similar to those our ancestors knew, while modern viticulture and oenology practices still refer to those of the ancient Greeks, preserved and later evolved by the Roman Empire [14].

The wine matrix has one of the most complex compositions, with more than 800-1000 volatile compounds ranging from alcohols, esters, acids, ketones, ethers, aldehydes, terpenes, lactones, phenolic compounds, etc., that arise from the grape and/or are formed during the fermentation process, with concentrations from a few parts per billion to a few percent in weight [14-17]. The abundance of these compounds depends on many factors: grape variety and origin, viticulture practices (blending, aging, yeast used during fermentation), production treatment, storage and oxygen exposure [17-19].

From the analytical point of view, wine analysis is a challenging task due to the complexity of the mixture and, moreover, minor differences in the concentration of certain compounds lead to wines with completely different organoleptic characteristics. Furthermore, the synergy between groups of compounds often has a stronger influence on the organoleptic characteristics than individual compounds [17].

Over the last few years, food control has become more appreciated by consumers. They have a right to expect that the foods they consume will be safe and of high quality. The term food 
control includes a large number of factors such as: safety, setting standards for toxicological and microbiological hazards, implementing procedures and practices to ensure those standards; nutrition, ensuring the necessary nutrient levels and formulation in foods that contribute to a healthy diet; quality, which provides the expected sensory characteristics in foods, such as taste, aroma and/or appearance; and finally value, giving the consumers utility and economic advantages. Some of these factors, such as value, are exclusively in the domain of industry and consumers; whereas others, such as safety, are shared interests of government, industry and consumers [20].

Although there are several methods to determine quality parameters of interest in the wine industry, they are usually long, tedious and require very expensive laboratory equipment and qualified personnel. Moreover, the quality control that evaluates the organoleptic properties of wines, such as flavor, taste and color, is usually tested by a trained, expert human panel (winetasters) turning out a strong subjective element in the results that are influenced by their skills, mood and/or physical state [14]. Moreover, the number of tests that panelists can perform is limited to a few assessments per day, due to the saturation of tongue receptors. As time is a critical factor in the food industry, it is necessary to develop new, simple and fast analytic tools able to assess antioxidant activity and organoleptic properties, as well as the authentication of wines and grapes and geographic origin verification. These factors are becoming very important due to wine regulations, which include rules concerning the marketing, production, designations of origin and geographical indications, among others, becoming more restrictive than in the past with the final objective of protecting consumers in order to supply beverages and food that are safe, of good quality and exempt from fraud.

On the other hand, analytical tools for determining grape and wine characteristics are critical for oenologists. The standard analytical methods of alcoholic beverages, including wine, are based on traditional chemical analysis and on wet chemistry procedures or instrumental methods, involving such separation techniques as high-performance liquid chromatography (HPLC) or gas chromatography (GC) combined with mass spectrometry (MS), and such optical techniques as Fourier transform infrared spectroscopy (FTIR) [17,21-23].

A variety of new analytical tools are increasingly used to profile the volatile, non-volatile and elemental composition of grapes and wines in order to analyze aroma, taste, color, mouthfeel and even for authentication. Methods range from classical chemical analysis through to the use of modern analytical instrument techniques. For example, some improvements in standard 
analytical tools that have been implemented are: gas chromatography combined with tandem mass spectrometry (GC-MS/MS), which offers significant advantages for trace quantification of aroma-active volatiles and contaminant compounds [24,25]; the ultra-high performance liquid chromatography-quadrupole time-of-flight mass spectrometry (UHPLC/Q-TOF-MS) technique is a new approach in the chromatographic separations used for profiling non-volatiles and for varietal, geographic and vintage authentication purposes [26,27]; inductively coupled plasma mass spectrometry (ICP-MS) has been used to analyze metals that affect the chemical stability and oxidative reactions in wines [28]; nuclear magnetic resonance spectroscopy (NMR) has become popular for characterizing and profiling primary and secondary metabolites $[29,30]$; isotope ratio mass spectrometry (IRMS) is applied to detect the authentication of wines [31]; while, in connection with methods using IRMS, stable isotope ratio analysis (SIRA), based on the measurement of the ratios of the stable isotopes of carbon $\left({ }^{13} \mathrm{C} / 12 \mathrm{C}\right)$, hydrogen $\left({ }^{2} \mathrm{H} /{ }^{1} \mathrm{H}\right)$, and oxygen $\left({ }^{18} \mathrm{O} / 16 \mathrm{O}\right)$ within molecules and between different molecules, is one of the most powerful analytical tools for the authentication of wine [32,33]; and finally, gas chromatography combined with olfactometry (GC-0) for the characterization of aroma qualities of complex mixtures [34].

Table 1 collects the analytical methods, including botho classical and modern, traditionally used in the analytical chemistry of wine [24-37]. Although these techniques are sophisticated and provide accurate results, they only give information on individual wine components, but they cannot give a global assessment of the flavor or quality of wines that is produced by the synergistic interaction between several chemical compounds present in the complex chemical wine matrix $[15,17]$. Moreover, the equipment used is costly, large, requires calibration procedures, sample preparation, and the process is time-consuming and needs skilled and qualified personnel. 
Table 1. Overview of classical chemical analysis and modern analytical instrument techniques usually applied to test wines.

\begin{tabular}{|c|c|c|}
\hline Analysis & Compounds/feature & Analytical method \\
\hline \multirow{11}{*}{$\begin{array}{l}\text { Characterization } \\
\text { of wines }\end{array}$} & pH levels & pH meter \\
\hline & Acid levels or tritatable acidity & $\begin{array}{l}\text { - Classical titration (with an indicator or } \\
\text { potentiometric titration) } \\
\text { - Photometry }\end{array}$ \\
\hline & Sugar levels & $\begin{array}{l}\text { - Refractive index (RI) } \\
\text { - Ion chromatography (IC) } \\
\text { - FT-NIR, FT-IR }\end{array}$ \\
\hline & Nitrogen & $\begin{array}{l}\text { - Ammonia ion selective electrode } \\
\text { - Enzymatic assay }\end{array}$ \\
\hline & Ethanol & $\begin{array}{l}\text { - Enzymatic assay } \\
\text { - Gas chromatography (GC) } \\
\text { - FT-NIR, FT-IR }\end{array}$ \\
\hline & Glycerol & $\begin{array}{l}\text { - Enzymatic assay } \\
\text { - FT-NIR, FT-IR }\end{array}$ \\
\hline & Acetaldehyde & $\begin{array}{l}\text { - Enzymatic assay } \\
\text { - Liquid chromatography (LC) }\end{array}$ \\
\hline & Sulphite & $\begin{array}{l}\text { - Distillation } \\
\text { - Classical titration (iodometric or } \\
\text { potentiometric) } \\
\text { - Photometry } \\
\text { - Flow Injection Analysis (FIA) } \\
\text { - IC }\end{array}$ \\
\hline & Organic acids & - Ion chromatography (IC) \\
\hline & Metal contaminants (Cu, Zn, Fe) & $\begin{array}{l}\text { - Atomic absorption spectroscopy (AAS) } \\
\text { - ICP-MS }\end{array}$ \\
\hline & Pesticides & $\begin{array}{l}\text { - GC-MS } \\
\text { - LC-MS/MS }\end{array}$ \\
\hline \multirow[t]{8}{*}{$\begin{array}{l}\text { Authentication } \\
\text { of wine and } \\
\text { fraud detection }\end{array}$} & $\begin{array}{l}\text { Chaptalization (adding sugar to increase } \\
\text { ethanol content) }\end{array}$ & $\begin{array}{l}\text { SIRA }\left({ }^{2} \mathrm{H} /{ }^{1} \mathrm{H}-\text { methyl and methylene in }\right. \\
\text { ethanol) } \\
\left({ }^{13} \mathrm{C} \text { in sugars/ethanol) }\right.\end{array}$ \\
\hline & Sugars (beet, cane and mixtures) & $\begin{array}{l}\text { SIRA }\left({ }^{2} \mathrm{H} /{ }^{1} \mathrm{H} \text {-methyl and methylene in }\right. \\
\text { ethanol } \\
\left({ }^{13} \mathrm{C} \text { in sugars/ethanol) }\right.\end{array}$ \\
\hline & Water (addition) & $\begin{array}{l}\text { SIRA }\left({ }^{18} \mathrm{O} \text { water }{ }^{2} \mathrm{H} /{ }^{1} \mathrm{H} \text {-methylene in }\right. \\
\text { ethanol) }\end{array}$ \\
\hline & Glycerol (synthetic or animal) & SIRA $\left({ }^{13} \mathrm{C}\right.$ glycerol) \\
\hline & Carbon dioxide (synthetic or fossil) & SIRA ( ${ }^{13} \mathrm{C}$ carbon dioxide) \\
\hline & Tartaric acid (synthetic) & SIRA $\left({ }^{13} \mathrm{C}\right.$ tartaric acid $)$ \\
\hline & Geographical origin mislabeling & $\begin{array}{l}\text { - SIRA }\left({ }^{2} \mathrm{H} /{ }^{1} \mathrm{H} \text {-methyl and methylene in }\right. \\
\text { ethanol } \\
\left({ }^{13} \mathrm{C} \text { ethanol }{ }^{18} \mathrm{O} \text { water }\right) \\
-{ }^{1} \mathrm{H}-\mathrm{NMR}\end{array}$ \\
\hline & Vintage mislabeling & $\begin{array}{l}\text { - SIRA }\left({ }^{2} \mathrm{H} /{ }^{1} \mathrm{H} \text {-methyl and methylene in }\right. \\
\text { ethanol } \\
\text { - SIRA }\left({ }^{13} \mathrm{C} \text { ethanol }{ }^{18} \mathrm{O} \text { water }\right)\end{array}$ \\
\hline
\end{tabular}


In order to reduce the cost and time-consumption of the previously described analytical procedures, electronic tongues (e-tongues) have emerged as a new analytical tool to assess a global, rapid and easy response with information concerning parameters related to the quality and organoleptic properties of grapes and wines, such as the phenolic content. E-tongues based on electrochemical sensors (potentiometric, amperometric, voltammetric or impedimetric) have been developed and used in food quality control [6,39-40]. In this sense, electrochemical techniques can lead to advantages in analytical chemistry due to their higher sensitivity and relatively low cost in comparison with the chromatography and spectroscopic methods, and they can be used as an alternative and/or complementary tool to the standard methods of analysis $[41,42]$.

In addition, compared to conventional tasting panels, electronic tongues have the clear advantage that they can be applied in continuous-flow for quality control in industrial processes; whereas an expert taster cannot evaluate samples on a continuous basis. Table 2 shows the main advantages of electronic tongues over the use of tasting panels, traditional chemical analysis (gas or liquid chromatography, spectroscopy techniques, etc.) and modern analysis with high generation equipment (GC-MS/MS, UHPLC/Q-TOF-MS, ICP-MS, SIRA).

The development of new analytical tools includes the manufacture of portable devices conceived for use by wine farmers in situ in the vineyards. In this way, wine farmers could test their grapes with a simple, rapid experiment in the vineyard in order to decide the optimal point of maturity for harvesting grapes with no need to test samples in the oenological laboratory.

Finally, e-tongue technology has already been successfully applied to analyze such beverages as wines, beers and strong alcoholic beverages (spirits and liqueurs) as well as to evaluate the quality of non-alcoholic beverages [43-46]. 
Table 2. Advantages of electronic tongues over other methodologies.

\begin{tabular}{lcccc}
\hline \hline & $\begin{array}{c}\text { Electronic } \\
\text { tongue }\end{array}$ & $\begin{array}{c}\text { Classical } \\
\text { chemical } \\
\text { analysis }\end{array}$ & $\begin{array}{c}\text { Modern } \\
\text { analytical } \\
\text { instrument } \\
\text { techniques }\end{array}$ & $\begin{array}{c}\text { Tasting } \\
\text { panels }\end{array}$ \\
\hline Simplicity & Yes & No & No & Yes \\
\hline Design or acquisition costs & Low & Medium & High & High \\
\hline Time consumption in measurements & Low & High & High & Medium \\
\hline Need for highly qualified personnel & No & No & Yes & Yes \\
\hline $\begin{array}{l}\text { Contamination/ } \\
\text { Destruction of the sample }\end{array}$ & No & Yes & Yes & Yes \\
\hline Generation of waste & Low & High & Low & High \\
\hline Possibility of on-site measurement & Yes & Yes & No & No \\
\hline $\begin{array}{l}\text { Possibility of incorporating the } \\
\text { measuring system into production } \\
\text { lines (e.g., food industry) }\end{array}$ & Yes & No & No & No \\
\hline
\end{tabular}

\subsection{Overview of research}

This research investigates the development of a quite new analytical tool, called electronic tongue, to analyze grapes and wines in terms of quality, vintage and/or geographical origin. The manufacture of new electrochemical sensors needs appropriate sensing materials able to interact with antioxidants, as well as making sensors with cross-selectivity and good stability in complex mixtures such as wines and grape musts. Therefore, two lines of research are adopted in this research. The first is basic research leading to the study of different sensing materials with interesting characteristics, such as electrocatalytic properties, to be used in the manufacture of new sensors, mainly based on thin films. The sensors were prepared by means of thin film manufacturing techniques such as spin-coating, electrodeposition and/or Layer by Layer. A range of sensing materials such as phthalocyanines, conducting polymers and nanoparticles are used in the construction of the electrochemical sensors. Enzymes have also been considered in this study to develop biosensors that will provide a certain specificity to the sensors.

In the second line of research, the as-manufactured sensors and biosensors were used to construct electronic and/or bioelectronic tongues to test standard solutions of interest in the food industry and to analyse real samples of wines and grapes. For this purpose, the study has counted on the support of important wineries that have provided wine and grape samples, as well as their chemical parameters analysed by means of standard methods. These collaborations have been of vital importance for this research, since it has been possible to establish correlations between the experimental data obtained with the electronic and/or bioelectronic 
tongues and the chemical parameters provided by the wineries. For this purpose, a variety of multivariate data analyses have been applied. Princial component analysis (PCA) and parallel factor analysis (PARAFAC) have been used to discriminate between grape and wine samples, partial least squares (PLS-1) has been applied to construct linear regression models between the electrochemical data obtained with the electronic and bioelectronics tongues and the chemical parameters of interest of grapes and wines, such as the phenolic content, sugar content, alcohol degree, etc.; and finally, support vector machines (SVM) have been implemented to predict the quality parameters of wines from the data obtained in the grapes analyses.

In Chapter 3, an introduction to each paper is provided, discussing such topics as the advantages of the sensing materials used in this work, the techniques used to prepare the sensors, the sensitivity offered by the sensors and biosensors, the electrochemical technique used for the experimental acquisition of data, the expected results and the applied multivariate statistical analysis.

\subsection{Research objectives}

The research objectives proposed for the dissertation are:

1. To design and construct electronic and bioelectronic tongues specifically dedicated to quality analysis for wines and grapes using chemically modified electrochemical sensors.

2. To improve sensor performance by means of (a) new electrocatalytic materials, (b) sensors based on nanostructured films, (c) combining different electrocatalytic materials to increase the electrochemical capability of the sensors and (c) introducing enzymes to enhance sensor specificity to detect phenolic compounds and/or sugars.

3. To implement different surface modification techniques for sensing materials immobilization (e.g., electrodeposition, spin-coating and/or Layer by Layer) with the purpose of improving the sensitivity, reproducibility and/or life-time of sensors.

4. To apply different electrochemical techniques (e.g., voltammetric, impedimetric, electrochemical quartz crystal microbalance, etc.) to evaluate their versatility.

5. To discover ways to combine enzymes with nanomaterials, such as metal nanoparticles, using strong interactions. 
6. To validate the electronic tongue as a tool for monitoring grape ripening from véraison to the optimal maturity date.

7. To implement the system for varietal authentication purposes in order to discriminate grape and red wine samples.

8. To establish correlations between the results obtained with the electronic and/or bioelectronic tongue in real samples of wines and grapes with the chemical parameters measured with traditional, standard analytical methods.

9. To evaluate the capability of the system to predict quality parameters of wines from the data obtained with the grapes used to elaborate wines. 


\subsection{References}

[1] Y. Vlasov, A. Legin, A. Rudnitskaya, C. Di Natale, A. D'Amico. Nonspecific sensor arrays ("electronic tongue") for chemical analysis of liquids (IUPAC Technical Report). Pure and Applied Chemistry 77 (2005) 1965-1983.

[2] D. Kirsanov, O. Mednova, V. Vietoris, P.A. Kilmartin, A. Legin. Towards reliable estimation of an "electronic tongue" predictive ability from PLS regression models in wine analysis. Talanta 90 (2012) 109-116.

[3] P. Oliveri, M. Chiara Casolino, M. Forina. Chemometric brains for artificial tongues. Advances in Food and Nutrition Research 61 (2010) 57-117.

[4] T.T. Bachmann, R.D. Schmid. A disposable multielectrode biosensor for rapid simultaneous detection of the insecticides paraoxon and carbofuran at high resolution. Analytica Chimica Acta 401 (1999) 95-103.

[5] E. Tonning, S. Sapelnikova, J. Christensen, C. Carlsson, M. Winther-Nielsen, E. Dock, R. Solna, P. Skladal, L. Norgaard, T. Ruzgas, J. Emneus. Chemometric exploration of an amperometric biosensor array for fast determination of wastewater quality. Biosensors and Bioelectronics 21 (2005) 608-617.

[6] M.L. Rodríguez-Méndez. Electronic Noses and Tongues in the Food Science. Elsevier: Amsterdam, 2016.

[7] Spectral Engines. Industry 4.0 and how smart sensors make the difference. March 20, 2018.

https://www.spectralengines.com/articles/industry-4-0-and-how-smart-sensors-make-the-difference.

Accessed July 16, 2019.

[8] J. LePree. Smart sensors enable Industry 4.0. May 1, 2019.

https://www.chemengonline.com/smart-sensors-enable-industry-4-0/

Accessed July 16, 2019.

[9] OIV, International Organisation of Vine and Wine. OIV Statistical Report on World Vitiviniculture. 2018 World Vitiviniculture Situation.

[10] M. Quiñones, M. Miguel, A. Aleixandre. Beneficial effects of polyphenols on cardiovascular disease. Pharmacological Research 68 (2013) 125-131.

[11] K. Chatzianagnostou, S. Del Turco, A. Pingitore, L. Sabatino, C. Vassalle. The Mediterranean lifestyle as a nonpharmacological and natural antioxidant for healthy aging. Antioxidants 4 (2015) 719-736.

[12] K. Griffiths, B.B. Aggarwal, R.B. Singh, H.S. Buttar, D. Wilson, F. De Meester. Food antioxidants and their antiinflammatory properties: a potential role in cardiovascular diseases and cancer prevention. Diseases 4 (2016) 1-15.

[13] B. Halliwell. Antioxidant and anti-inflammatory components of foods in ILSI Europe Concise Monograph Series. P. Calder (Ed.). ILSI Europe: Brussels, 2015. pp. 34-44.

[14] C.G. Branchini, L. Lvova, C. Di Natale, R. Paolesse. Wine and combined electronic nose and tongue in Electronic Noses and Tongues in Food Science. M.L. Rodriguez Mendez (Ed.). Elsevier: Amsterdam, 2016. pp. 301307.

[15] M.L. Rodríguez Méndez, J.A. De Saja, C. Medina-Plaza, C. García-Hernández. Electronic tongues for the organoleptic characterization of wines in Electronic Noses and Tongues in Food Science. M.L. Rodriguez Mendez (Ed.). Elsevier: Amsterdam, 2016. pp. 265-273.

[16] M. Vilanova, Z. Genisheva, M. Graña, J.M. Oliveira. Determination of Odorants in Varietal Wines from International Grape Cultivars (Vitis vinífera) Grown in NW Spain. South African Journal for Enology and Viticulture 34 (2013) 212-222.

[17] R.S. Jackson. Wine science: Principles and applications. Elsevier: Amsterdam, 2014

[18] J. Wirth, C. Morel-Salmi, J.M. Souquet, J.B. Dieval, O. Aagaard, S. Vidal, H. Fulcrand, V. Cheynier. The impact of oxygen exposure before and after bottling on the polyphenolic composition of red wines. Food Chemistry 123 (2010) 107-116.

[19] V. Atasanova, H. Fulcrand, V. Cheynier, M. Moutounet. Effect of oxygenation on polyphenol changes occurring in the course of wine-making. Analytica Chimica Acta 458 (2002) 15-27.

[20] S. Gardner. Consumers and food safety: A food industry perspective in Food, Nutrition and Agriculture. J.L. Albert (Ed.). Food and Agriculture Organization (FAO), 1993. 
[21] A.M.A. Borbalán, L. Zorro, D.A. Guillén, C. García Barroso. Study of the polyphenol content of red and white grape varieties by liquid chromatography-mass spectrometry and its relationship to antioxidant power. Journal of Chromatography A 1012 (2003) 31-38.

[22] A.A. De la Cruz, G. Hilbert, C. Riviere, V. Mengin, N. Ollat, L. Bordenave, S. Decroocq, J.C. Delaunay, S. Delrot, J.M. Merillon, J.P. Monti, E. Gomes, T. Richard. Anthocyanin identification and composition of wild Vitis spp. accessions by using LC-MS and LC-NMR. Analytica Chimica Acta 732 (2012) 145-152.

[23] M. Gishen, R.G. Dambergs, D. Coxxolino. Grape and wine analysis - enhancing the power of spectroscopy with chemometrics. A review of some application in the Australian wine industry. Australian Journal of Grape and Wine Research 11 (2005) 296-305.

[24] I.C.C. Nóbrega, G.E. Pereira, M. Silva, E.V.S. Pereira, M.M. Medeiros, D.L. Telles, E.C. Albuquerque Jr., J.B. Oliveira, D.W. Lachenmeier. Improved sample preparation for GC-MS-SIM analysis of ethyl carbamate in wine. Food Chemistry 177 (2015) 23-28.

[25] C. Thibon, A. Pons, N. Mouakka, P. Redon, R. Méreau, P. Darriet. Comparison of electron and chemical ionization modes for the quantification of thiols and oxidative compounds in white wines by gas chromatographytandem mass spectrometry. Journal of Chromatography A 1415 (2015) 123-133.

[26] A. Vallverdú-Queralt, N. Boix, E. Piqué, J. Gómez- Catalan, A. Medina-Remon, G. Sasot, M. Mercader-Martí, J.M. Llobet, R.M. Lamuela-Raventos. Identification of phenolic compounds in red wine extract samples and zebrafish embryos by HPLC-ESI-LTQ-Orbitrap-MS. Food Chemistry 181 (2015) 146-151.

[27] C. Barnaba, E. Dellacassa, G. Nicolini, T. Nardin, M. Malacarne, R. Larcher. Identification and quantification of 56 targeted phenols in wines, spirits, and vinegars by online solid-phase extraction-ultrahigh-performance liquid chromatography-quadrupole-Orbitrap mass spectrometry. Journal of Chromatography A 1423 (2015) 124-135.

[28] D. Kruzlicova, Ž. Fiket, G. Kniewald. Classification of Croatian wine varieties using multivariate analysis of data obtained by high resolution ICP-MS analysis. Food Research International 54 (2013) 621-626.

[29] K. Skogerson, R. Runnebaum, G. Wohlgemuth, J. de Ropp, H. Heymann, O. Fiehn. Comparison of gas chromatography-coupled time-of-flight mass spectrometry and $1 \mathrm{H}$ nuclear magnetic resonance spectroscopy metabolite identification in white wines from a sensory study investigating wine body. Journal of Agricultural and Food Chemistry 57 (2009) 6899-6907.

[30] C. Fotakis, K. Kokkotou, P. Zoumpoulakis, M. Zervou. NMR metabolite fingerprinting in grape derived products: An overview. Food Research International 54 (2013) 1184-1194.

[31] B. Raco, E. Dotsika, D. Poutoukis, R. Battaglini, P. Chantzi. O-H-C isotope ratio determination in wine in order to be used as a fingerprint of its regional origin. Food Chemistry 168 (2015) 588-594.

[32] G. Versini, F. Camin, M. Ramponi, E. Dellacassa. Stable isotope analysis in grape products: ${ }^{13} \mathrm{C}$-based internal standardization methods to improve the detection of some types of adulterations. Analytica Chimica Acta 563 (2006) $325-330$.

[33] N. Dordevic, F. Camin, R.M. Marianella, G.J. Postma, L.M.C. Buydens, R. Wehrens. Detecting the addition of sugar and water to wine. Australian Journal of Grape and Wine Research 19 (2013) 324-330.

[34] A.J. Johnson, G.D. Hirson, S.E. Ebeler. Perceptual characterization and analysis of aroma mixtures using gas chromatography recomposition-olfactometry. PLOS ONE 7 (2012) 1-7.

[35] V. Di Egidio, N. Sinelli, G. Giovanelli, A. Moles, E. Casiraghi. NIR and MIR spectroscopy as rapid methods to monitor red wine fermentation. European Food Research and Technology 230 (2010) 947-955.

[36] J. Oliva, G. Martinez, S. Cermeno, M. Motas, A. Barba, M.A. Camara. Influence of matrix on the bioavailability of nine fungicides in wine grape and red wine. European Food Research and Technology 244 (2018) 1083-1090.

[37] B. Prusisz, K. Mulica, P. Pohl. Ion exchange and ion exclusion chromatographic characterization of wines using conductivity detection. Journal of Food and Drug Analysis 16 (2008) 95-103.

[38] A. Riul Jr., C.A.R. Dantas, C.M. Miyazaki, O.N. Oliveira Jr. Recent advances in electronic tongues. Analyst 135 (2010) 2481-2495.

[39] H. Jiang, M. Zhang, B. Bhandari, B. Adhikari. Application of electronic tongue for fresh foods quality evaluation: A review. Food Reviews International 34 (2018) 746-769.

[40] M. Peris, L. Escuder-Gilabert. Electronic noses and tongues to assess food authenticity and adulteration. Trends in Food Science and Technology 58 (2016) 40-54.

[41] E. Bakker, Y. Qin. Electrochemical Sensors. Analytical Chemistry 78 (2006) 3965-3983. 
[42] R. Sandulescu, M.Tertis, C. Cristea, E. Bodoki. New materials for the construction of electrochemical biosensors in Biosensors - Micro and Nanoscale Applications. T. Rinken (Ed.). IntechOpen: London, 2015.

[43] A. Rudnitskaya, L.M. Schmidtke, A. Reis, M.R.M. Domingues, I. Delgadillo, B. Debus, D. Kirsanov, A. Legin. Measurements of the effects of wine maceration with oak chips using an electronic tongue. Food Chemistry 229 (2017) 20-27.

[44] M. Ghasemi-Varnamkhasti, M.L. Rodriguez-Mendez, S.S. Mohtasebi, C. Apetrei, J. Lozano, H. Ahmadi, S.H. Razavi, J.A. de Saja. Monitoring the aging of beers using a bioelectronic tongue. Food Control 25 (2012) 216-224.

[45] W. Novakowski, M. Bertotti, T.R.L.C. Paixao. Use of copper and gold electrodes as sensitive elements for fabrication of an electronic tongue: Discrimination of wines and whiskies. Microchemical Journal 99 (2011) 145-151.

[46] F. Winquist, J. Olsson, M. Eriksson. Multicomponent analysis of drinking water by a voltammetric electronic tongue. Analytica Chimica Acta 683 (2011) 192-197. 

Doctoral Thesis: Electronic tongue technology applied to the analysis of grapes and wines

\section{CHAPTER 2}

\section{LITERATURE REVIEW}





\subsection{Introduction to electronic tongues}

The main taste evaluation method in the food industry currently consists of a sensory analysis in which experienced evaluators, called sensory panelists, test the samples for evaluation [1-6]. However, in wine tastings, these analyses have some drawbacks, such as low objectivity and reproducibility, saturation of tongue receptors, as well as the high stress imposed on the panelists. Moreover, cross-mouth contamination between samples can compromise the validity of the sensory results [7-10].

A new sensory technology has been developed to evaluate the taste of food and beverages in an objective way. It is called the electronic tongue and it consists of a multisensor system of low selective sensors and uses advanced mathematical procedures for signal processing based on pattern recognition and/or multivariate analysis [11]. The concept of the electronic tongue was first developed by Toko, Vlasov and Winquist [12-14], although the first works concerning these systems were published mainly by Toko and Hayashi using the term multichannel taste sensors [15-17].

Electronic tongues are systems inspired to detect taste in similar manner to the human gustatory sensation [18]. The human tongue is equipped with different receptors (taste-buds) able to distinguish thousands of substances. The information on taste substances provided by the receptors is transduced into an electric signal and sensory nerves are responsible for transmitting it to the brain and then, our biological computer, trained with knowledge acquired earlier, is able to identify what we eat and also perceive information about the quantity of individual compounds (such as the amount of salt or sucrose). The performance of the taste system is attributed to the capability of taste-buds to respond to different stimuli, called cross-selectivity, and to the ability of analyzing the signal from all the tongue receptors at once. These two qualities (cross-selectivity and multivariate analysis) are the key concepts involving electronic tongue systems. In electronic tongues, sensors play the role of taste receptors and the brain is substituted by a computer equipped with appropriate data-processing techniques (Figure 1) [8].

As stated before, the IUPAC defines an electronic tongue as a multisensor system, which consists of a number of low selective sensors and uses advanced mathematical procedures for signal processing based on the pattern recognition (PARC) and/or multivariate analysis [artificial neural networks (ANNs), principal component analysis (PCA), etc [11]. In addition, many research groups working in this topic have summarized the definition of an electronic tongue as an analytical instrument comprising an array of non-specific, poorly selective chemical sensors with 
partial specificity (cross sensitivity) to different compounds in a solution, coupled to an appropriate chemometric tool for data processing [19-21].

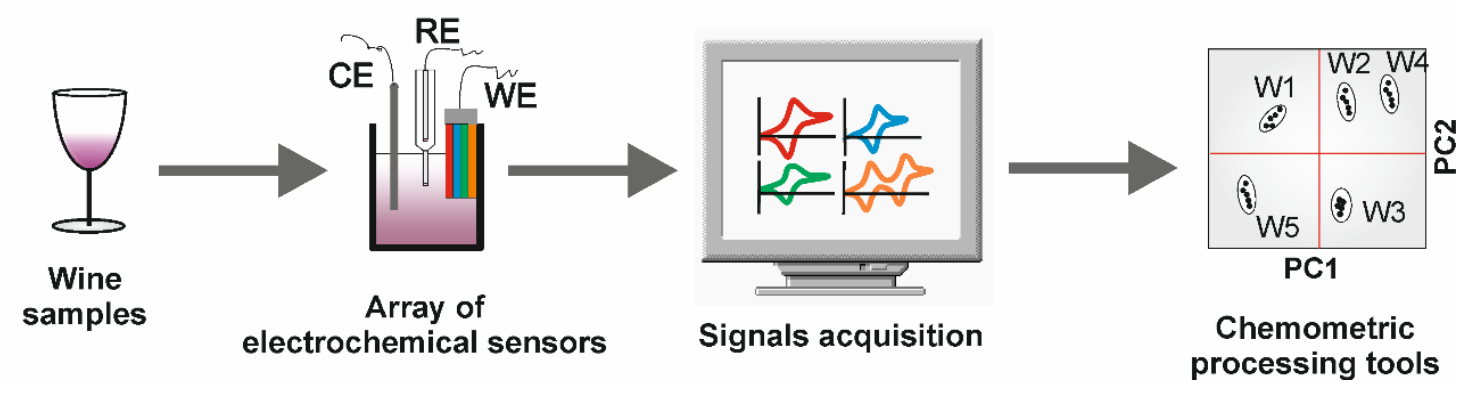

Figure 1. Schematic presentation of the electronic tongue system.

Although the concept of chemical sensor is generally used to detect a chemical substance at high sensitivity, human taste receptors do not necessarily recognize individual chemical substances. Each of the receptors for the five basic tastes simultaneously receives multiple chemical substances, showing a semi-selective response. It is practically impossible to measure the taste of foods containing hundreds of taste substances by chemical analysis methods, such as gas or liquid chromatography; although these techniques can measure the concentration of chemical substances. Therefore, in electronic tongues, sensors do not give the amounts of specific molecules that contribute to a certain taste, but rather the taste quality and intensity, in other words, a global evaluation of the analyzed matrix. Moreover, a taste sensor should be able to measure such interactions between taste substances as the synergistic effect and the suppression effect. The discrimination of each chemical substance is not an important task here, but recognition of the taste itself is, and its quantitative expression is necessary $[8,11,13,18,19,22]$.

Arrays of sensors in electronic tongues combine non-specific chemical sensors with partial sensitivity that provides responses with cross-sensitivity. The difference in the electrical response of different sensors serves as a fingerprint for the analyzed sample. When combining these systems with pattern recognition software, we use the fingerprints of different samples to discriminate or classify them.

In the 1990s, while research was being carried out on the biological and molecular processes involved in taste receptors, sensitive technology was also being developed for objective evaluation in the discrimination and/or quantification of tastes, prior to the discoveries of taste receptors. Thus, in 1989, Toko and co-workers applied for a patent for their potentiometric taste sensor. They developed a taste sensor equipped in a multichannel electrode system using 
an immobilized lipid membrane with a polymer as transducer [17]. This taste sensor is considered to be the first electronic tongue with global selectivity and has been applied to the analysis of numerous beverages such as beer, sake, coffee, tea or milk, among others [19].

After this first electronic tongue, and up to the present time, numerous papers have been published on this technology, mainly applied to the analysis of food and beverages, as it is considered a novel technology that can contribute greatly to quality purposes. Thus, in last few years, the design and application of these new systems has become very popular. One of the most important research groups in this field, which can also be considered as a pioneer in the design of electronic tongues, is Winquist and co-workers. In 1997, Winquist and Lundström developed an electronic tongue based on voltammetric techniques for the analysis of fruit juices, beverages and milk. They were able to detect the aging process of milk and orange juice with the developed system $[14,23]$. Following this work, in 2000, they combined potentiometry, voltammetry and conductivity measurements to produce a hybrid electronic tongue for the analysis of fermented milk [24]. Selective ion electrodes were used to measure $\mathrm{pH}$, carbon dioxide and chlorine ion concentration. The electronic tongue consisted of six working electrodes of different metals. In 2002, Winquist and collaborators developed a measurement system based on the flow injection analysis technique applied to a voltammetric electronic tongue for the analysis of different standard solutions and for the classification of different apple juices [25].

Another research group dedicated to the design of electronic tongues since the 1990s is led by Prof. Vlasov, who together with the group of Prof. Di Natale and Paolesse, have made important advances in the development of potentiometric electronic tongues. In 1997, this group applied solid-state selective ion potentiometric sensors based on chalcogenide glass in an electronic tongue to detect heavy metal ions in solutions [26], in the analysis of beverages (tea, beer, coffee, juices, soft drinks, etc.) [27] and polluted waters [28]. Later, between 2003 and 2007, they published some works in which these electrodes were applied to the analysis of mineral waters, wines and alcoholic beverages $[29,30]$.

One of the first works based on a voltammetric electronic tongue based on chemically modified electrodes was developed in 2003-2004 in the Laboratory of Inorganic Chemistry and Physical Chemistry of the University of Valladolid, directed by Prof. Rodríguez-Méndez. This electronic tongue was based on sensors prepared with graphite powder mixed with phthalocyanine and it was applied to the analysis of wine [31,32]. There are currently several international research groups of experts in electronic tongues that have made important 
advances in the design and application of these systems in the field of food and beverages [3350].

In the case of the wine industry, it is important to monitor changes occurring during grape ripening, wine fermentation and aging to ensure the quality of the wines, to assess the origin and authentication and to avoid falsifications or adulterations. Traditional techniques for analysing food and beverages, as well as human tasting panels, have some drawbacks and need new analytical tools. In this sense, electronic tongues are reliable and reproducible analytical tools, which have demonstrated their utility for the rapid and inexpensive assessment of different chemical matrices, including wines.

\subsection{Principles of detection: Electrochemical methods}

The heart of an electronic tongue is the array of sensors which captures physical or chemical signals from its surroundings and converts them into electrical signals. The sensors used in electronic tongues are mainly optical, electrochemical and gravimetric. In food analysis applications, the most commonly used are the electrochemical sensors (potentiometric, amperometric, voltammetric and impedimetric), due to their simple instrumentation, high sensitivity, fast response and ease of operation [51-53].

In potentiometric sensors, the voltage is measured at null current. A working electrode covered with a membrane is introduced into the sample to be analyzed. The potential is created at the membrane/solution interface, which is needed to retain the balance of the electrochemical process and depends on the nature of the electrode material and the composition of the solution. The advantages of potentiometric electronic tongues are the well-known principle of operation, low cost, ease of commercial production and possibility of obtaining selective sensors. Their disadvantages are the influence of the temperature on the measurements and the adsorption of solution components on the electrode surfaces that can modify the potentials. These sensors can be prepared from different materials, membranes and techniques $[22,53,54]$.

In voltammetric/amperometric sensors, the measurements are based on the electric current established between the working electrode and the reference electrode in an electrochemical cell. In voltammetric sensors, a polarization voltage is applied while the current is measured. When a potential change occurs, the current flows through the working electrode, thus oxidizing or reducing the compound under analysis. The response current is the result of the 
electrochemical reaction that occurs at the electrode/electrolyte interface layer. Voltamograms show peaks associated with the oxidation and reduction of electroactive species from the solution, and the intensity is a function of the analyte concentration. Moreover, voltammograms can show peaks associated to the electroactive material deposited on the electrode surface. In amperometric sensors, voltammetry is applied as a preliminary step to study the oxidation potentials of the analyte, and once established, amperometric measurements are carried out to measure the electrical current between the electrodes as a function of a constant potential applied to the working electrode. The current intensity signal from the oxidation or reduction of the substance analyzed is proportional to its concentration. These sensors are sensitive, very versatile and different materials can be used in the electrodes for sensing. Moreover, using voltammetric sensors, different techniques can be applied (cyclic voltammetry, linear sweep voltammetry, differential pulse voltammetry or square-wave voltammetry) [53,55-57]. The main disadvantage of such sensors is the lack of selectivity.

The impedimetric sensors, which are considered part of the group of electrochemical methods, are based on impedance spectroscopy measurements. The main advantage over the previous sensors is that there is no need for a reference electrode. In this method, the impedance is measured at one constant frequency or for a frequency spectrum. This system can be described by an equivalent electrical circuit, in which the electrostatic double-layer formed at the electrode/electrolyte interface governs the response at low frequencies, the solution conductance and ultrathin films coating the electrodes rule the total impedance at intermediate frequencies and the geometric capacitance is most relevant at high frequencies. The advantage of these sensors is their high sensitivity $[22,53]$. They could be employed in an electronic tongue; however, in practice, the works reporting impedimetric electronic tongues are quite rare compared with potentiometric, amperometric or voltammeric systems.

Table 3 shows some examples of electrochemical electronic tongues based on potentiometric, amperometric, voltammetric and impedimetric sensors applied to food analysis. Hybrid systems have also been developed, mainly combining voltammetric and potentiometric sensors. 
Table 3. Examples of electrochemical electronic tongues applied to food analysis.

\begin{tabular}{|c|c|c|c|}
\hline "Application & Sample & Type of e-tongue & Reference \\
\hline \multirow[t]{7}{*}{ Food process monitoring } & \multirow[t]{2}{*}{ Red wine } & Potentiometric & \multirow{2}{*}{$58-60$} \\
\hline & & Voltammetric & \\
\hline & \multirow[t]{2}{*}{ Beer } & Potentiometric & \multirow{2}{*}{46,61} \\
\hline & & Voltammetric & \\
\hline & \multirow{2}{*}{ Milk } & Potentiometric & \multirow{2}{*}{62,63} \\
\hline & & Voltammetric & \\
\hline & Cheese & Potentiometric & 64 \\
\hline \multirow[t]{9}{*}{ Food quality and freshness } & Meat & Potentiometric & 37 \\
\hline & \multirow{2}{*}{ Fish } & Potentiometric & \multirow{2}{*}{$65,66,67$} \\
\hline & & Voltammetric & \\
\hline & $\begin{array}{l}\text { Non-alcoholic } \\
\text { beverages }\end{array}$ & Potentiometric & 48 \\
\hline & Milk & Voltammetric & 68 \\
\hline & Rice & Potentiometric & 69 \\
\hline & Olive oils & Voltammetric & 33 \\
\hline & Tea & Voltammetric & 34 \\
\hline & Apples & Hybrid & 49 \\
\hline \multirow[t]{20}{*}{ Authentication } & \multirow[t]{3}{*}{ Red wines } & Voltammetric & \multirow{3}{*}{$70-72$} \\
\hline & & Potentiometric & \\
\hline & & Impedimetric & \\
\hline & \multirow[t]{3}{*}{ Beer } & Potentiometric & \multirow{3}{*}{$50,73-75$} \\
\hline & & Voltammetric & \\
\hline & & Hybrid & \\
\hline & \multirow[t]{2}{*}{ Alcoholic beverages } & Potentiometric & \multirow{2}{*}{$30,76,77$} \\
\hline & & Voltammetric & \\
\hline & \multirow[t]{2}{*}{ Milk } & Potentiometric & \multirow{2}{*}{78} \\
\hline & & Voltammetric & \\
\hline & Yogurt & Voltammetric & 80 \\
\hline & Honey & Potentiometric & 81,82 \\
\hline & Drinking water & Potentiometric & 42 \\
\hline & \multirow[t]{3}{*}{ Juices } & Potentiometric & \multirow{3}{*}{$14,38,83$} \\
\hline & & Voltammetric & \\
\hline & & Hybrid & \\
\hline & \multirow[t]{2}{*}{ Tea } & Potentiometric & \multirow{2}{*}{84,85} \\
\hline & & Voltammetric & \\
\hline & Olive oils & Voltammetric & 86 \\
\hline & Apples & Potentiometric & 87 \\
\hline
\end{tabular}




\subsection{Distinct materials for the sensing units}

Sensing materials used for electrochemical sensors can be classified as: 1) materials for the electrode and supporting substrate, 2) materials for improving electroanalytical performances, 3) materials for the immobilization of biological recognition elements and 4) biological elements, the last two being applicable to electrochemical biosensors. Sensing units of traditional electronic tongues are mainly lipid membranes, ion-selective electrodes or noble metals functioning as working electrodes, with the voltage difference measured between the sensing unit and a reference electrode [22]. The choice of materials for the sensing units is crucial to obtain a high performance. Even though e-tongues do not require specific interactions with the analyte, the sensing units need to respond electrically to small changes in the liquid under analysis. Moreover, depending on the method of detection, materials with electrical conductivity and/or electroactivity might be required. Materials used for the electrode and supporting substrate are usually conductive materials exhibiting low currents in an electrolyte solution, free of any electroactive species, over a relatively wide potential window. Although these sensing materials play different roles in different electrochemical sensing systems, depending on their properties, their basic functions are mainly grouped as: 1) enhancement of electron transfer, 2) catalysis of electrochemical reactions, 3) immobilization of biomolecules, 4) labelling biomolecules and 5) acting as reactant.

Lipid membranes were one of the first class of materials used in potentiometric sensors for e-tongues to mimic the materials of the human tongue [13]. In addition, chalcogenide glasses were largely used in electrochemical measurements due to the ease of electrode preparation and cross-sensitivity [28]. However, due to the extensive use of electrochemical methods, research began to explore electroactive materials. Among the most frequently used materials for the electrode and supporting substrate, the following may be mentioned: phthalocyanines, porphyrins, metal nanoparticles, metal oxide nanoparticles, carbon-based materials and conducting polymers.

In recent years, conducting polymers (CPs), which consist of organic m-conjugated systems, have been employed in electrochemical sensors due to their light weight, workability, resistance to corrosion, low cost and excellent electrical, mechanical, optical and conducting properties $[88,89]$. CPs are generally semi-conductive in nature and have low conductivity, but can be made more conductive by doping. This means that the oxidation and reduction of a $\pi$ conjugated system generates $\mathrm{p}$ - and n-doped CPs. The doping process can be controlled by 
chemical and electrochemical methods. Therefore, to overcome their low conductivity, composites of CPs have extensively been studied and applied in electrochemical applications [88]. Conducting polymers such as polypyrrole, polyaniline, PEDOT, or polythiophene have been used as sensing materials to construct electrochemical sensors [90-95].

Other sensing materials for electrochemical sensors are metallophthalocyanines (MPcs) and metalloporphyrins (MPs), which are organometallic compounds, also known as N4macrocyclic metal complexes. They contain N4-ligands that co-ordinate to the metal ions. In their nature, the N4-ligands of phthalocyanine and porphyrin are 18- $\pi$ conjugated ring systems with a highly conjugated structure. Metal ions are from the transition metals, group I-V metals, lanthanides and actinides. These MPc and MP complexes have a very rich electrochemistry owing to their ability to accept and donate electrons, either from central metal ions or 18-тconjugated ring systems $[96,97]$. Phthalocyanines and porphyrins have been extensively used as sensing materials in electrochemical sensors due to their remarkable electrochemical and electrocatalytic properties [98-105].

Important advances have been made recently in the field of sensors thanks to the introduction of new platforms for sensors design, such as nanotechnological materials and nanostructured architectures (i.e., nanoparticles, carbon nanotubes and nanofibres, graphenes, nanostructured surfaces, etc.), which have improved their sensitivity. Many kinds of nanoparticles, including metal nanoparticles, oxide nanoparticles, semiconductor nanoparticles and even composite nanoparticles have been widely used in electrochemical sensors and biosensors. These nanoparticles exhibit unique optical, electrical, thermal and catalytic properties. Nanoparticles with different sizes and compositions are now available for electroanalysis applications. Metal nanoparticles have shown excellent conductivity and catalytic properties, which make them suitable for acting as "electronic wires" to enhance the electron transfer between redox centers in proteins and electrode surfaces and as catalysts to increase electrochemical reactions. Oxide nanoparticles are often used to immobilize biomolecules due to their biocompatibility while semiconductor nanoparticles are often used as labels or tracers for electrochemical analysis [106-110]. The use of nanoparticles applied to electrochemical sensors has been widely reported [106-114].

The combination of more than one sensing material to make composite materials can show excellent sensing and catalytic properties. The advantage of the synergistic interaction between different sensing materials produces a higher performance of electrochemical sensors. 
This effect has been observed in the interaction between gold nanoparticles and nickel phthalocyanine [115]. A synergetic effect between silver-gold nanoparticles and zinc phthalocyanine has also been demonstrated in the electrocatalytic reduction of $\mathrm{O}_{2}$ on vitreous carbon electrodes [116]. Gold nanoparticles-ZnO composite is one of the most widely discussed electrocatalytic materials with high catalytic activity, optical sensitivity, universal biocompatibility and high chemical stability [117]. Pt nanoparticles and PANI aero-gel have demonstrated a synergetic effect that results in the immobilization of a high density of GOx for an efficient oxidation of glucose [118].

Biochemical sensors are currently being successfully introduced into electronic tongue technology (bioelectronic tongues) by adding enzymes as a biorecognition element to the electrode surfaces. The biorecognition elements are classified into biological (enzymes, whole cells, cell organelles, tissue) and artificial (biomimetic) receptors (aptamers, ribosymes and moleculary imprinted polymers (MIPs)) [119]. Biosensors based on enzymes combine the advantages of classical electrochemical sensors, which provide high sensitivity and crossselectivity, with the typical specificity of the biosensors $[120,121]$. In these biosensors, the selectivity for the target analyte is mainly determined by the biorecognition element, whereas the sensitivity of the biosensor is greatly influenced by the transducer [119]. Therefore, the electron transfer between the active sites of the enzyme and the electrode surface can be improved through an electronic mediator, such as the above mentioned sensing materials, acting as an electrical wire. Specific enzymes for the detection of phenols (e.g., tyrosinase, laccase or peroxidase) or sugars (e.g., glucose oxidase or fructose dehydrogenase) among others, have been incorporated into bioelectronic tongues [44,106,107,109,119-122].

\subsection{Sensor and biosensor construction}

Electrochemical sensors can be chemically modified with sensing materials by means of several techniques to improve their electroanalytical performance. E-tongue technology covers different types of sensors and biosensors, from classical sensors prepared by means of simple techniques (carbon paste electrodes, casting, electrodeposition, etc.) [31,32,123-127] to sophisticated and costly nanostructured sensors, including techniques of preparation such as self-assembled monolayer (SAM), Layer by Layer (LbL) or Langmuir-Blodgett (LB) [128-134]. 
Therefore, the major challenge in the field of electrochemical sensors, consisting in the improvement of their electroanalytical performance, as already mentioned, was addressed by using nanotechnology, which includes not only the use of sensing nanomaterials, such as nanoparticles, carbon nanotubes or graphene, providing higher electrocatalytic properties, but also the preparation of nanostructured architectures, where sensing materials can be deposited as nanostructured thin films. Sensors based on thin films at the nanoscale show high surface-tovolume ratio and small dimensions. The high surface ratio facilitates the interaction between the sensing material and the analyte, which leads to improvements in the sensitivity, and the small dimensions enable fast adsorption and desorption kinetics for the analyte in the sensing material and, therefore, providing a rapid response time [135].

\section{Classical methods}

Carbon paste-based methods: sensors are prepared by mixing a carbon-based material (graphite, carbon nanotubes, graphene, carbon nanoparticles) with the sensing material and a mineral oil. The carbon matrix acts as a support to prevent agglomeration and provides a large surface area, high mechanical strength, fast electron transfer rate, excellent thermal and electrical conductivities, which in turn enhance the electrocatalytic activity. These sensors are cheap, easy to prepare and have the great advantage that they can be easily recovered by cleaning the surface with a filter paper [31,32].

Coating-based methods: drop-casting, dip-coating or spin-coating are the most commonly used coating methods for modifying electrodes with nanomaterials. These methods are easy and low-cost in sensor manufacture and do not require complicated instruments. However, only soluble or solution-processible nanomaterials can be deposited onto an electrode surface [136].

Direct deposition-based methods: these methods are diverse, and include electrochemical, electrospinning, electrospray, sputtering and vapor deposition. Electrochemical deposition is one of the most used methods to deposit thin-films of polymers or polymer composites onto conducting electrode surfaces. This technique can be performed at room temperature and is carried out using three electrodes dipped into the solution at a potential (or current, depending on the method used) that is applied to polymerize the monomer and/or reduce metal ions in the simultaneous preparation of polymer composites with metal nanoparticles [136]. 
Printing-based methods: these methods include the well-known screen-printed method as well as newly developed methods, such as inkjet printing, nozzle-jet printing or the laserscribing process. Printing-based methods have been used to deposit materials onto rigid and flexible substrates to make devices on a large-scale at low cost. One advantage of these methods is that they allow nanomaterials to be desposited directly onto predesigned patterns [136].

\section{Methods based on nanostructured architectures}

Self-assembled monolayer (SAM): this technique is used to form monomolecular films by the spontaneous adsorption of amphiphilic adsorbates in an orderly manner onto a solid support. It has been widely used to coat electrode surfaces. The initial driving force for the assembly is the chemical affinity between the adsorbates and the substrate. In the initial stage of SAMs, mainly alkyl compounds, such as alkylated thiols and silanols were used to construct well-organized SAM structures. However, there are various functional groups that can be used to generate SAMs [137].

Langmuir-Blodgett (LB): these films are formed by the deposition of a Langmuir film onto a solid substrate. Langmuir films are composed of an organized monolayer of amphiphilic molecules at a liquid/gas interface. These molecules with their hydrophobic tail and hydrophilic head are spontaneously oriented at a liquid/gas interface. Unlike the SAM technique, the LB method can provide multi-layered film structures with defined thickness and sequence, together with well-ordered molecular arrangement and orientation [137].

Layer by Layer $(L b L)$ : these films are formed by depositing onto a substrate alternating layers of oppositely charged materials. This technique has been given much attention as a versatile method for nanostructured films. Most of the LbL assemblies are based on electrostatic interactions between the layered materials. However, $L b L$ assemblies can be driven by other interactions, such as hydrogen bonding, metal interactions, charge transfer complexation or biospecific recognition, among others. A wide range of materials can be applied in the assembling process, such as polymers, biomaterials, supramolecular assemblies and inorganic substances. This method only requires the use of beakers and tweezers in the procedure [137].

\section{Methods used for biosensor construction}

The immobilization of enzymes to construct biosensors depends on the materials and procedure used and can include: 1) entrapment behind a permeable membrane as a thin film 
covering the electrochemical detector; 2) entrapment within a conducting polymeric matrix or solgel; 3) entrapment within self-assembled monolayers or within LbL layers; 4) immobilization onto an LB structure; 5) covalent bonding on membranes or surfaces activated by means of bifunctional groups or spacers (glutaraldehyde, carbodiimide, silanization, etc.); 6) bulk modification of the electrode material (graphite epoxy resin or modified carbon paste, clays, zeolites, nanomaterials, organic polymers, complex compounds, etc.) $[44,101,112,122,137-$ 141].

\subsection{Methods of data analysis}

The correct utilization of e-tongues requires an appropriate data analysis. The main task of these systems is to classify the samples under study $[8,22,53]$. As the number of samples may be very large and many measurements are needed to distinguish between very similar samples whose variability may also be considerable for complex liquids such as wines, juices, etc.- an enormous and complex amount of data is generated that must be processed using chemometric or pattern recognition methods. Four stages can be distinguished during data analysis: signal preprocessing, dimensionality reduction, prediction and validation [8]. Data pre-processing is carried out mainly to scale the data, discard outliers and extract representative parameters. Subsequently, a dimensionality reduction of variables is needed in many cases, for example when using cyclic voltammetry, since the information obtained is large and complex. Then, one solution is to simplify the high dimensionality, maintaining the meaningful information from the data and suppressing redundant and non-significant information without loss of information by using a feature extraction stage, such as Legendre polynomials, Fourier transform, kernel method, wavelet transformation or genetic algorithms. Depending on the type of application, different methods for dimensionality reduction can be applied. The most used process in the literature for e-tongues is principal component analysis (PCA), a non-supervised method used to discriminate between samples with different characteristics. PCA plots can be obtained in 2 or 3 dimensions (2D or $3 \mathrm{D})$, depending on the need to distinguish the samples. This tool reduces the number of variables to new latent variables (principal components) in a reduced variable space to facilitate identification or classification. In addition, this new space simplifies the interpretation of the variability contained in the raw information. Therefore, PCA is a very powerful non-supervised linear pattern recognition method that reduces the dimensionality of multivariate data and helps to 
visualize the different categories of multivariate profiles with high similarities and differences between sample clusters.

When the number of classes of samples is very large, for instance if one wishes to distinguish between a set of dozens of wines, the amount of points placed leads to overcrowding the $2 \mathrm{D}$ or 3D PCA plot. Hence, other methods to treat the data are required [22]. In this context, methods involving artificial neural networks (ANNs), parallel factor analysis (PARAFAC), soft independent modelling of class analogy (SIMCA), linear discriminant analysis (LDA) or support vector machines (SVM) are used.

The low dimensional feature may also be used for prediction and/or regression purposes, where the sensors respond as independent variables and a second set of variables (e.g., chemical parameters of the samples) as dependent variables are used to establish regression models that will be able to predict and validate some parameters of the analyzed samples. These models are usually established using partial least squares (PLS), principal component regression (PCR) or support vector machines regression (SVMR), among others.

\subsection{Summary of the applications}

Electronic tongues have several applications in various industrial areas, mainly in the pharmaceutical and food industry. In the pharmaceutical industry, it has been widely used to quantify the taste-making efficiency of medicines, as well as to analyze the stability of medicines regarding taste $[142,143]$. In addition, e-tongues have also been applied to the field of medicine for non-invasive diagnostics (urine, sweat, saliva, etc.) or clinical analysis $[144,145]$, and in the field of the environment to analyze water contamination $[146,147]$.

In the food industry, this new technology has been used in quality control during production, in food storage stages, in the control of aging processes, in the analysis of tastes, to evaluate authentication, to identify brand and/or geographical origin, or to detect adulterations (see Table 3).

Given the advantages and numerous applications of electronic tongue technology, many efforts have been made to make miniaturized and commercial prototypes of electronic tongue devices at a low cost. In this field, the main issues to overcome are miniaturization and the integration with existing silicon technology for microelectronics [22]. The first commercial 
electronic tongues, SA402B and TS-5000Z Taste Sensing Systems, based on 7 potentiometric electrodes with lipid-polymeric membranes, were developed by Toko and co-workers (Intelligent Sensor Technology Inc., Japan) $[13,19]$. These devices have been used to determine and quantify the intensity of taste in food products and pharmaceuticals [148-152].

Another commercial e-tongue is Astree II (Alpha MOS, France), which is based on 7 ionselective field effect transistors (ISFETs) and has been applied to quality control, food recognition, taste assessment, process monitoring and the pharmaceutical industry [152-156]. Other existing commercial systems, although their applications are rather scarce compared with those already mentioned, are: the Multiarray Chemical Sensor (McScience Inc., Korea) built of PVC and polyurethane membranes selective to ions $\left(\mathrm{H}^{+}, \mathrm{Na}^{+}, \mathrm{Ca}^{2+}, \mathrm{NH}_{4}{ }^{+}, \mathrm{NO}_{3}{ }^{-}, \mathrm{Cl}-\right)$ [157] and Sensor System (St. Petersburg, Russia) comprising 7 potentiometric ion-selective sensors [158].

\subsubsection{Electronic tongues in the wine industry}

The wine production process, as shown, comprises a series of steps that need to be carefully managed and for which continuous checking and measurements are required. Electronic tongues have been applied to the study of grapes, musts and wines in different stages of the winemaking process.

The chemical composition of wines has a direct influence on their organoleptic properties. Each wine has a different chemical composition that depends on the variety and state of ripeness of the grapes, the extraction of different components in the must and the reactions that occur during the winemaking process, the post-fermentation treatment and the aging of wine. The ripeness and quality of the grapes used to elaborate wines is usually established on their external appearance or the taste of the grape juices. The analysis of sugar and phenolic content is also a common practice to establish the quality $[20,159]$.

Electronic tongues have been applied to discriminate between grapes according to their variety and vintage [44]. After crushing, during maceration, the contact between the must and

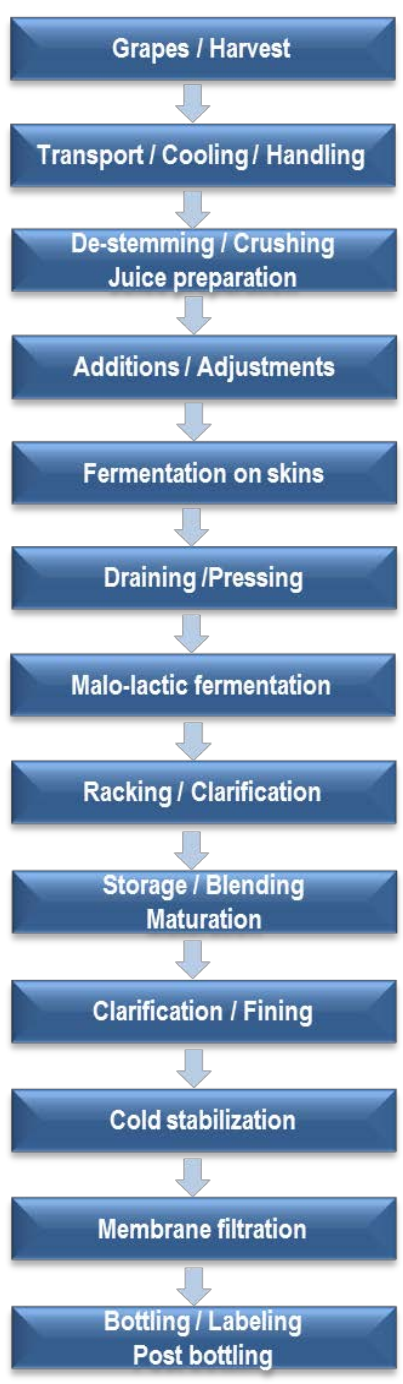


the skins increases the concentration of phenols. Moreover, by applying different pressing techniques, the extraction of phenols can be increased. E-tongues have been used to study the increase in phenol concentration after using Flash Release and micro-oxygenation pressing techniques [160].

The fermentation of fresh grape juice or must transforms sugars into alcohols, producing wine. In white wines, only one fermentation is needed, which is the alcoholic fermentation producing ethanol, whilst red wines need two fermentation processes, the alcoholic fermentation followed by the malolactic fermentation, where malic acid is transformed into lactic acid. Here, the control by means of chemical sensors is quite difficult, because fermentation is a turbulent process that produces changes in temperature that affect the performance of the sensors. For this reason, only a few papers have described the use of e-tongues to monitor wine fermentation. Using infrared spectroscopy, combined with an e-nose and e-tongue, the kinetics of the fermentation process of musts has been analyzed and acceptable correlations with sugar consumption and alcohol production have been found [35].

After the fermentation of the grape must, the final product, the wine, is obtained. It is then aged in oak barrels for 1-2 years before bottling. This stage has a great influence on the organoleptic characteristics that wine acquires, influenced by the retention and release of volatile compounds from the lees during ageing. The oxygen that diffuses through the small pores of the barrels, the origin of the oak wood and its degree of toasting also influence the final taste of the wine. Subsequently, the wine continues to be aged in bottles in a reducing environment that further improves its organoleptic properties. Electronic tongues have been applied to discriminate red wines according to their ageing [161] and the origin of oak barrels and toasting level $[58,162]$.

In addition, as the ageing stage in barrels requires a lot of time and high costs, innovative techniques are applied to accelerate the ageing. One of these techniques consists of adding pieces of wood of different sizes in stainless steel tanks with built-in micro-oxygenation. An electronic tongue has been applied to discriminate between wines aged by traditional techniques and wines aged with alternative practices [60].

After ageing, the wines are bottled. Traditionally, the bottles are sealed with stoppers made of natural cork, ideal for sealing the liquid while allowing oxygenation from the outside through small pores. Traditional natural cork stoppers are associated with high quality wines. Nowadays, the wine industry uses synthetic stoppers, made of polymers, with a degree of 
porosity. The effect of oxygen transfer in synthetic closures has been studied using an electronic tongue $[59,160]$.

The organoleptic properties of final wines produced with different varieties of grape, vintage, appellation, etc., have been evaluated with e-tongues. The grapes used to make wine will determine both the taste and the final organoleptic characteristics of the product. There are many grape varieties and each winery uses different oenological techniques to produce different styles of wine with different flavours and characteristics. An electronic tongue has been used to discriminate wines with different Spanish appellations (Rioja, Rueda, Ribera de Duero) [31] and, in addition, this e-tongue was also used to determine differences in these wines from vintage to vintage [57]. Bioelectronic tongues have also been used to analyze cava wines [163] or to discriminate grape juices prepared with different varieties of grape [44].

Weather conditions or improper storage, among other variables, can spoil the final wine. E-tongues have been used to monitor the spoilage of wines by monitoring their acidity levels [164].

Finally, e-tongues have been used to evaluate possible adulterations or frauds in wines $[70,165]$. Some adulteration practices consist in dilution with water, blending with wine of lower quality or made with grapes from a different region, the use of prohibited ageing techniques and the addition of ethanol or other substances (tartaric acid, tannic acid, $\mathrm{SO}_{2}$, acetic acid or sucrose). 


\subsection{References}

[1] M.R. Hood White, H. Heymann. Assessing the sensory profiles of sparkling wine over time. American Journal of Enology and Viticulture 66 (2015) 156-163.

[2] L.W. Lucherk, T.G. O'Quinn, J.F. Legako, R.J. Rathmann, J.C. Brooks, M.F.Miller. Consumer and trained panel evaluation of beef strip steaks of varying marbling and enhancement levels cooked to three degrees of doneness. Meat Science 122 (2016) 145-154.

[3] H.S. Kwak, B.H. Ahn, H.R. Kim, S.Y. Lee. Identification of sensory attributes that drive the likeability of Korean rice wines by American panelists. Journal of Food Science 80 (2015) 161-170.

[4] M. Kortesniemi, S. Rosenvald, O. Laaksonen, A. Vanag, T. Ollikka, K. Vene, B.R. Yang. Sensory and chemical profiles of Finnish honeys of different botanical origins and consumer preferences. Food Chemistry 246 (2018) 351359.

[5] M. McKay, F.F. Bauer, V. Panzeri, A. Buica. Testing the sensitivity of potential panelists for wine taint compounds using a simplified sensory strategy. Foods 7 (2018) 1-14.

[6] M. Ojeda, I. Etaio, L. Guerrero, M.P. Fernandez-Gil, F.J. Perez-Elortondo. Does consumer liking fit the sensory quality assessed by trained panelists in traditional food products? A study on PDO Idiazabal cheese. Journal of Sensory Studies 33 (2018) 1-10.

[7] L. Lvova, I. Yaroshenko, D. Kirsanov, C. Di Natale, R. Paolesse, A. Legin. Electronic tongue for brand uniformity control: a case study of Apulian red wines recognition and defects evaluation. Sensors 18 (2018) 1-12.

[8] M.L. Rodríguez-Méndez. Electronic Noses and Tongues in Food Science. Elsevier: Amsterdam, 2016.

[9] D. Taladrid, L. Lorente, B. Bartolome, M.V. Moreno-Arribas, L. Laguna. An integrative salivary approach regarding palate cleansers in wine tasting. Journal of Texture Studies 50 (2019) 75-82.

[10] C.G. Branchini, L. Lvova, C. Di Natale, R. Paolesse. Wine and combined electronic nose and tongue in Electronic Noses and Tongues in Food Science. M.L. Rodriguez Mendez (Ed.). Elsevier: Amsterdam, 2016. pp. 301307.

[11] Y. Vlasov, A. Legin, A. Rudnitskaya, C. Di Natale, A. D'Amico. Nonspecific sensor arrays ("electronic tongue") for chemical analysis of liquids (IUPAC Technical Report). Pure and Applied Chemistry 77 (2005) 1965-1983.

[12] C. Di Natale, F. Davide, A.D'Amico, A. Legin, A. Rudinitskaya, B.L. Selezenev, Y. Vlasov. Applications of an electronic tongue to the environmental control. Technical digests of Eurosensors X, Leuven, Belgium, 1996, pp. 1345-1348.

[13] K. Toko. Taste sensor with global selectivity. Materials Science and Engineering C 4 (1996) 69-82.

[14] F. Winquist, P. Wide, I. Lundstrom. An electronic tongue based on voltammetry. Analytica Chimica Acta 357 (1997) 21-31.

[15] K. Hayashi, M. Yamanaka, K. Toko, K. Yamafuji. Multichannel taste sensor using lipid membranes. Sensors and Actuators B 2 (1990) 205-213.

[16] K. Toko, T. Matsuno, K. Yamafuji, K. Hayashi, H. Ikezaki, K. Sato, R. Toukubo, S. Kawarai. Multichannel taste sensor using electric potential changes in lipid membranes. Biosensors and Bioelectronics 9 (1994) 359-364.

[17] K. Toko, T., Murata, T. Matsuno, Y, Kikkawa, K. Yamafuji. Taste map of beer by a multichannel taste sensor. Sensors and Materials 4 (1992) 145-151.

[18] K. Toko. A taste sensor. Measurement Science and Technology 9 (1998) 1919-1936.

[19] Y. Tahara, K. Toko. Electronic Tongues - A review. IEEE Sensors Journal 13 (2013) 3001-3011.

[20] M.L. Rodriguez-Mendez, J.A. De Saja, R. Gonzales-Anton, C. Garcia-Hernandez, C. Medina-Plaza, C. GarciaCabezon, F. Martin-Pedrosa. Electronic Noses and Tongues in Wine Industry. Frontiers in Bioengineering and Biotechnology 4 (2016) 1-12.

[21] M. del Valle. Sensor arrays and electronic tongue systems. International Journal of Electrochemistry 2012 (2012) $1-11$.

[22] A. Riul Jr., C.A.R. Dantas, C.M. Miyazaki, O.N. Oliveira Jr. Recent advances in electronic tongues. Analyst 135 (2010) 2481-2495.

[23] P. Wide, F. Winquist, P. Bergsten, E.M. Petriu. The Human-based multisensor fusion method for artificial nose and tongue sensor data. IEEE Transactions on Instrumentation and Measurement 47 (1998) 1072-1077. 
[24] F. Winquist, S. Holmin, C. Krantz-Rulcker, P. Wide, I. Lundstrom. A hybrid electronic tongue. Analytica Chimica Acta 406 (2000) 147-157.

[25] F. Winquist, E. Rydberg, S. Holmin, C. Krantz-Rulcker, I. Lundstrom. Flow injection analysis applied to a voltammetric electronic tongue. Analytica Chimica Acta 471 (2002) 159-172.

[26] Y. Vlasov, A. Legin, A. Rudnitskaya. Cross-sensitivity evaluation of chemical sensors for electronic tongue: determination of heavy metal ions. Sensors and Actuators B 44 (1997) 532-537.

[27] A. Legin, A. Rudnistkaya, Y. Vlasov, C. Di Natale, F. Davide, A. D'Amico. Tasting of beverages using an electronic tongue. Sensors and Actuators B 44 (1997), 291-296.

[28] C. Di Natale, A. Macagnano, F. Davide, A. D'Amico, A. Legin, Y. Vlasov, A. Rudnitskaya, B. Selezenev. Multicomponent analysis on polluted waters by means of an electronic tongue. Sensors and Actuators B 44 (1997) 423-428.

[29] A. Legin, A. Rudnistkaya, L. Lvova, Y. Vlasov, C. Di Natale, A. D'Amico. Evaluation of Italian wine by the electronic tongue: recognition, quantitative analysis and correlation with human sensory perception. Analytica Chimica Acta 484 (2003) 33-34.

[30] A. Legin, A. Rudnistkaya, B. Seleznev, Y. Vlasov. Electronic tongue for quality assessment of ethanol, vodka and eau-de-vie. Analytica Chimica Acta 534 (2005) 129-135.

[31] V. Parra, T. Hernando, M.L. Rodriguez-Mendez, J.A. de Saja. Electrochemical sensor array made from bisphthalocyanine modified carbon paste electrodes for discrimination of red wines. Electrochimica Acta 49 (2004) 5177-5185.

[32] A. Arrieta, M.L. Rodriguez-Mendez, J.A. De Saja. Langmuir-Blodgett film and carbon paste electrodes based on phthalocyanines as sensing units for taste. Sensors and Actuators B 95 (2003) 357-365.

[33] I.M. Apetrei, C. Apetrei. Voltammetric e-tongue for the quantification of total polyphenol content in olive oils. Food Research International 54 (2013) 2075-2082.

[34] A. Ghosh, B. Tudu, P. Tamuly, N. Bhattacharyya, R. Bandyopadhyay. Prediction of theaflavin and thearubigin content in black tea using a voltammetric electronic tongue. Chemometrics and Intelligent Laboratory Systems 116 (2012) 57-66.

[35] S. Buratti, D. Ballabio, G. Giovanelli, C.M. Zuluanga Dominguez, A. Moles, S. Benedetti, N. Sinelli. Monitoring of alcoholic fermentation using near infrared and mid infrared spectroscopies combined with electronic nose and electronic tongue. Analytica Chimica Acta 697 (2011) 67-74.

[36] X. Cetó, F. Céspedes, M. del Valle. BioElectronic Tongue for the quantification of total polyphenol content in wine. Talanta 99 (2012) 544-551.

[37] L. Gil, J.M: Barat, D. Baigts, R. Martínez-Máñez, J. Soto, E. Garcia-Breijo, M.C. Aristoy, F. Toldrá, E. Llobet. Monitoring of physical-chemical and microbiological changes in fresh pork meat under cold storage by means of a potentiometric electronic tongue. Food Chemistry 126 (2011) 1261-1268.

[38] M. Gutierrez-Capitan, J.L. Santiago, J. Vila-Planas, A. Llobera, S. Boso, P. Gago, M.C. Martinez, C. JimenezJorquera. Classification and characterization of different white grape juices by using a hybrid electronic tongue. Journal of Agricultural and Food Chemistry 61 (2013) 9325-9332.

[39] L. Lvova, S. Denis, A. Barra, P. Mielle, C. Salles, C. Vergoignan, C. Di Natale, R. Paolesse, P. Temple-Boyer, G. Feron. Salt release monitoring with specific sensors in "in vitro" oral and digestive environments from soft cheeses. Talanta 97 (2012) 171-180.

[40] A.M. Peres, L.G. Dias, A.C.A. Veloso, S.G. Meirinho, J.S. Morais, A.A.S.C. Machado. An electronic tongue for gliadins semi-quantitative detection in foodstuffs. Talanta 83 (2011) 857-864.

[41] C.A. Blanco, R. De la Fuente, I. Caballero, M.L. Rodriguez-Mendez. Beer discrimination using a portable electronic tongue based on screen-printed electrodes. Journal of Food Engineering 157 (2015) 57-62.

[42] F. Winquist, J. Olsson, M. Eriksson. Multicomponent analysis of drinking water by a voltammetric electronic tongue. Analytica Chimica Acta 683 (2011) 192-197.

[43] M. Gay, E. Diez-Arevalo, M.L. Rodriguez-Mendez, J.A. De Saja. Electrochemical quartz crystal microbalance analysis of the oxidation reaction of phenols found in wines at lutetium bisphthalocyanine electrodes. Sensors and Actuators B 185 (2013) 24-31.

[44] C. Medina-Plaza, G. Revilla, R. Munoz, J.A. Fernandez-Escudero, E. Barajas, G. Medrano, J.A. de Saja, M.L. Rodriguez-Mendez. Electronic tongue formed by sensors and biosensors containing phthalocyanines as electron mediators. Application to the analysis of red grapes. Journal of Porphyrins and Phthalocyanines 18 (2014) 76-86. 
[45] L. Wu, H. Pu, D.W. Sun. Novel techniques for evaluating freshness quality attributes of fish: A review of recent developments. Trends in Food Science and Technology 83 (2019) 259-273.

[46] M. Ghasemi-Varnamkhasti, M.L. Rodríguez-Méndez, S.S. Mohtasebi, C. Apetrei, J. Lozano, H. Ahmadi, S.H. Razavi, J.A. de Saja. Monitoring the aging of beers using a bioelectronic tongue. Food Control 25 (2012) 216-224.

[47] Y. Tahara, K. Nakashi, K. Ji, A. Ikeda, K. Toko. Development of a portable taste sensor with a lipid/polymer membrane. Sensors 13 (2013) 1076-1084.

[48] L.G. Dias, A.M. Peres, T.P. Barcelos, J.S. Morais, A.A.S.C. Machado. Semi-quantitative and quantitative analysis of soft drinks using an electronic tongue. Sensor and Actuators B 154 (2011) 111-118.

[49] A. Kutyla-Olesiuk, M. Nowacka, M. Wesoly, P. Ciosek. Evaluation of organoleptic and texture properties of dried apples by hybrid electronic tongue. Sensors and Actuators B 187 (2013) 234-240.

[50] X. Ceto, J.M. Gutierrez, A. Mimendia, F. Cespedes, M. del Valle. Voltammetric electronic tongue for the qualitative analysis of beers. Electroanalysis 25 (2013) 1635-1644.

[51] M. Del Valle. Electronic tongues employing electrochemical sensors. Electroanalysis 22 (2010) 1539-1555.

[52] D.W. Kimmel, G. LeBlanc, M.E. Meschievitz, D.E. Cliffel. Electrochemical sensors and biosensors. Analytical Chemistry 84 (2012) 685-707.

[53]. M. Sliwinska, P. Wisniewska, T. Dymerski, J. Namiesnik, W. Wardencki. Food analysis using artificial senses. Journal of Agricultural and Food Chemistry 62 (2014) 1423-1448.

[54] P. Ciosek, W. Wróblewski. Potentiometric electronic tongues for foodstuff and biosample recognition-An overview. Sensors 11 (2011) 4688-4701.

[55] M. Scampicchio, D. Ballabio, A. Arecchi, S.M. Cosio, S. Mannino. Amperometric electronic tongue for food analysis. Microchimica Acta 163 (2008) 11-21.

[56] S. Cavanillas, F. Winquist, M. Eriksson. A self-polishing platinum ring voltammetric sensor and its application to complex media. Analytica Chimica Acta 859 (2015) 29-36.

[57] M.L. Rodriguez-Mendez, V. Parra, C. Apetrei, S. Villanueva, M. Gay, N. Prieto, J.A. de Saja. Electronic tongue based on voltammetric electrodes modified with materials showing complementary electroactive properties. Applications. Microchimica Acta 163 (2008) 23-31.

[58] A.A. Arrieta, M.L. Rodriguez-Mendez, J.A. De Saja. Voltametric electronic tongue application to wines classification and correlation study with the chemical and sensory characterization. Quimica Nova 33 (2010) 787793.

[59] A Rudnitskaya, L.M. Schmidtke, I. Delgadillo, A. Legin, G. Scollaryd. Study of the influence of micro-oxygenation and oak chip maceration on wine composition using an electronic tongue and chemical analysis. Analytica Chimica Acta 642 (2009) 235-245.

[60] M. Gay, C. Apetrei, I. Nevares, M. del Alamo, J. Zurro, N. Prieto, J.A. De Saja, M.L. Rodríguez-Mendez. Application of an electronic tongue to study the effect of the use of pieces of wood and micro-oxygenation in the aging of red wine. Electrochimica Acta 55 (2010) 6782-6788.

[61] A. Kutyła-Olesiuk, M. Zaborowski, P. Prokaryn, P. Ciosek. Monitoring of beer fermentation based on hybrid electronic tongue. Bioelectrochemistry 87 (2012) 104-113.

[62] M. Yoshida, H. Shinohara, T. Sugiyama, M. Kumagai, H. Muto, H. Kodama. Taste of milk from inflamed breasts of breastfeeding mothers with mastitis evaluated using a taste sensor. Breastfeeding Medicine 9 (2014) 92-97.

[63] Z. Wei, J. Wang, X. Zhang. Monitoring of quality and storage time of unsealed pasteurized milk by voltammetric electronic tongue. Electrochimicia Acta 88 (2013) 231-239.

[64] K. Esbensen, D. Kirsanov, A. Legin, A. Rudnitskaya, J. Mortensen, J. Pedersen, L. Vognsen, S. MakarychevMikhailov, Y. Vlasov, Y. Fermentation monitoring using multisensor systems: feasibility study of the electronic tongue. Analytical and Bioanalytical Chemistry 378 (2004) 391-395.

[65] L. Gil, J.M. Barat, I. Escriche, E. Garcia-Breijo, R. Martínez-Mañeez, J. Soto. An electronic tongue for fish freshness analisis using a thick-film array of electrodes. Microchimica Acta 163 (2008) 121-129.

[66] L. Gil, J.M: Barat, E. Garcia-Breijo, J. Ibañez, R. Martínez- Mañez, J. Soto, E. Llobet, J. Brezemes, J.C. Aristoy, F. Toldra. Fish freshness analysis using metallic potentiometric electrodes. Sensors and Actuators B 131 (2008) 362-370. 
[67] M.L. Rodríguez-Mendez, M. Gay, C. Apetrei, J.A. De Saja. Biogenic amines and fish freshness assessment using a multisensory system based on voltammetric electrodes. Comparison between CPE and screen-printed electrodes. Electrochimica Acta 54 (2009) 7033-7041.

[68] F. Winquist, C. Krantz-Rulcker, P. Wide, I. Lundström. Monitoring of freshness of milk by an electronic tongue on the basis of voltammetry. Measurement Science and Technology 9 (1998) 1937-1946.

[69] T.U. Tran, K. Suzuki, H. Okadome, S. Homma, K. Ohtsubo. Analysis of the tastes of brown rice and milled rice with different milling yields using a taste sensing system. Food Chemistry 88 (2004) 557-566.

[70] V. Parra, A.A. Arrieta, J.A. Fernandez-Escudero, M.L: Rodríguez- Mendez, J.A. De Saja. Electronic tongue based on chemically modified electrodes and voltammetry for the detection of adulterations in wines. Sensors and Actuators B 118 (2006) 448-453.

[71] C. Di Natale, R. Paolesse, A. Macagnano, A. Mantini, A. D’Amico, M. Ubigli, A. Legin, L. Lvova, A. Rudnitskaya, $Y$. Vlasov. Application of a combined artificial olfaction and taste system to the quantification of relevant compounds in red wine. Sensors and Actuators B 69 (2000) 342-347.

[72] A. Riul, H.C. de Sousa, R.R. Malmegrim, D.S. dos Santos, A.C.P.L.F. Carvalho, F.J. Fonseca, O.N. Oliveira, L.H.C. Mattoso. Wine classification by taste sensors made from ultra-thin films and using neural networks. Sensors and Actuators $B 98$ (2004) 77-82.

[73] J.M. Gutierrez, Z. Haddi, A. Amari, B. Bouchikhi, A. Mimendia, X. Ceto, M. del Valle. Hybrid electronic tongue based on multisensor data fusion for discrimination of beers. Sensors and Actuators B 177 (2013) 989-996.

[74] A. Rudnitskaya, E. Polshin, D. Kirsanov, J. Lammertyn, B. Nicolai, D. Saison, F.R. Delvaux, F. Delvaux, A. Legin. Instrumental measurement of beer taste attributes using an electronic tongue. Analytica Chimica Acta 646 (2009) 111-118.

[75] E. Polshin, A. Rudnitskaya, D. Kirsanov, A.Legin, D. Saison, F. Delvaux, F.R. Delvaux, B.M. Nicolaï, J. Lammertyn. Electronic tongue as a screening tool for rapid analysis of beer. Talanta 81 (2010) 88-94.

[76] M. Sliwinska, C. García-Hernández, M. Koscinski, T. Dymerski, W. Wardencki, J. Namiesnik, M. SliwinskaBartkowiak, S. Jurga, C. García-Cabezón, M.L. Rodríguez-Méndez. Discrimination of apple liqueurs (Nalewka) using a voltammetric electronic tongue, UV-Vis and Raman spectroscopy. Sensors 16 (2016) 1-14.

[77] W. Novakowski, M. Bertotti, T.R.L.C. Paixao. Use of copper and gold electrodes as sensitive elements for fabrication of an electronic tongue: discrimination of wines and whiskies. Microchemical Journal 99 (2011) 145-151.

[78] T.R.L.C. Paixao, M. Bertotti. Fabrication of disposable voltammetric electronic tongues by using Prussian Blue films electrodeposited onto CD-R gold surfaces and recognition of milk adulteration. Sensors and Actuators B 137 (2009) 266-273.

[79] L.A. Dias, A.M. Peres, A.C.A. Veloso, F.S. Reis, M. Vilas-Boas, A.A.S.C. Machado. An electronic tongue taste evaluation: Identification of goat milk adulteration with bovine milk. Sensors and Actuators B 136 (2009):209-217.

[80] Z. Wei, J. Wang, W. Jin. Evaluation of varieties of set yogurts and their physical properties using a voltammetric electronic tongue based on various potential waveforms. Sensors and Actuators B 177 (2013) 684-694.

[81] N. Major, K. Markovic, M. Krpan, G. Šaric, M. Hruskar, N. Vahčc. Rapid honey characterization and botanical classification by an electronic tongue. Talanta 85 (2011) 569-574.

[82] I. Escriche, M. Kadar, E. Domenech, L Gil-Sanchez. A potentiometric electronic tongue for the discrimination of honey according to the botanical origin. Comparison with traditional methodologies: physicochemical parameters and volatile profile. Journal of Food Engineering 109 (2012) 449-456.

[83] P. Ciosek, R. Maminska, A. Dybko, W. Wroblewski. Potentiometric electronic tongue based on integrated array of microelectrodes. Sensors and Actuators B 127 (2007) 8-14.

[84] W. He, X. Hu, L. Zhao, X. Liao, Y. Zhang, M. Zhang, J. Wu. Evaluation of Chinese tea by the electronic tongue: correlation with sensory properties and classification according to geographical origin and grade level. Food Research International 42 (2009) 1462-1467.

[85] P. Ivarsson, S. Holmin, N.E. Hojer, C. Krantz-Ruilcker, F. Winquist. Discrimination of tea by means of a voltammetric electronic tongue and different applied waveforms. Sensors and Actuators B 76 (2001) 449-454

[86] C. Apetrei, I.M. Apetrei, S. Villanueva, J.A. de Saja, F. Gutierrez-Rosales, M.L. Rodriguez-Mendez. Combination of an e-nose, an e-tongue and an e-eye for the characterisation of olive oil with different degree of bitterness. Analytica Chimica Acta 663 (2010) 91-97. 
[87] A. Rudnitskaya, D. Kirsanov, A. Legin, K. Beullens, J. Lammertyn, B.M. Nicolai, J. Irudayaraj. Analysis of apples varieties - comparison of electronic tongue with different analytical techniques. Sensors and Actuators B 116 (2006) 23-28.

[88] M.H. Naveen, N.G. Gurudatt, Y.B. Shim. Applications of conducting polymer composites to electrochemical sensors: A review. Applied Materials Today 9 (2017) 419-433.

[89] H. Yoon. Current trends in sensors based on conducting polymer nanomaterials. Nanomaterials 3 (2013) 524549.

[90] W. Wang, G. Xu, X.T. Cui, G. Sheng, X. Luo. Enhanced catalytic and dopamine sensing properties of electrochemically reduced conducting polymer nanocomposite doped with pure graphene oxide. Biosensors and Bioelectronics 58 (2014) 153-156.

[91] D. Lakshmi, A. Bossi, M.J. Whitcombe, I. Chianella, S.A. Fowler, S.Subramanyam, E.V. Piletska, S.A. Piletsky. Electrochemical sensor for catechol and dopamine based on a catalytic molecularly imprinted polymer-conducting polymer hybrid recognition element. Analytical Chemistry 81 (2009) 3576-3584.

[92] Y.S. Gao, J.K. Xu, L.M. Lu, L.P. Wu, K.X. Zhang, T. Nie, X.F. Zhu, Y. Wu. Overoxidized polypyrrole/graphene nanocomposite with good electrochemical performance as novel electrode material for the detection of adenine and guanine. Biosensors and Bioelectronics 62 (2014) 261-267.

[93] H. Jiang, A. Zhang, Y. Sun, X. Ru, D. Ge, W. Shi. Poly(1-(2-carboxyethyl)pyrrole)/polypyrrole composite nanowires for glucose biosensor. Electrochimica Acta 70 (2012) 278-285.

[94] L. Kergoat, B. Piro, D.T. Simon, M.C. Pham, V. Noel, M. Berggren. Detection of glutamate and acetylcholine with organic electrochemical transistors based on conducting polymer/platinum nanoparticle composites. Advanced Materials 26 (2014) 5658-5664.

[95] G. Fabregat, E. Armelin, C. Aleman. Selective detection of dopamine combining multilayers of conducting polymers with gold nanoparticles. The Journal of Physical Chemistry B 118 (2014) 4669-4682.

[96] J. Zagal, F. Bedioui, J.P:Dodelet. N4-Macrocyclic Metal Complexes. Springer: New York, 2006.

[97] D. Dini, M. Hanack. Physical properties of phthalocyanine-based materials. In The Porphyrin Handbook, Phthalocyanines: Properties and Materials, Vol. 17. K. Kadish, K. Smith, R. Guilard (Eds.). Elsevier: Amsterdam, 2003.

[98] Y. Sivalingam, R. Pudi, L. Lvova, G. Pomarico, F. Basoli, A. Catini, A. Legin, R. Paolesse, C. Di Natale. The light modulation of the interaction of L-cysteine with porphyrins coated ZnO nanorods. Sensors and Actuators B 2019 (2015) 613-621.

[99] A. Legin, S. Makarychev-Mikhailov, O. Goryacheva, D. Kirsanov, Y. Vlasov. Cross-sensitive chemical sensors based on tetraphenylporphyrin and phthalocyanine. Analytica Chimica Acta 457 (2002) 297-303.

[100] L. Lvova, R. Paolesse, C. Di Natale, A. D'Amico. Detection of alcohols in beverages: An application of porphyrin-based Electronic tongue. Sensors and Actuators B 118 (2006) 439-447.

[101] M.L. Rodriguez-Mendez, C. Medina-Plaza, J.A. de Saja, C. Apetrei, R. Muñoz. Sensor arrays based on phthalocyanines: New developments on nanostructured and biomimetic electrochemical sensors. In L. Lvova, D. Kirsanov, C. Di Natale, A. Legin (Eds.), Multisensor systems for chemical analysis: Materials and Sensors. Pan Stanford Publishing: Singapore, 2012; 70-109.

[102] J.H. Zagal, S. Griveau, J.F. Silva, T. Nyokong, F. Bedioui. Metallophthalocyanine-based molecular materials as catalysts for electrochemical reactions. Coordination Chemistry Reviews 254 (2010) 2755-2791.

[103] P. Kalimuthu, A. Sivanesan, S.A. John. Fabrication of optochemical and electrochemical sensors using thin films of porphyrin and phthalocyanine derivatives. Journal of Chemical Sciences 124 (2012) 1315-1325.

[104] M.L. Rodriguez-Mendez, M. Gay, J.A. de Saja. New insights into sensors based on radical bisphthalocyanines. Journal of Porphyrins and Phthalocyanines 13 (2009) 1159-1167.

[105] K.Y. Hou, L. Huang, Y.B. Qi, C.X. Huang, H.B. Pan, M.A. Du. Bisphenol A sensor based on novel selfassembly of zinc phthalocyanine tetrasulfonic acid-functionalized graphene nanocomposites. Materials Science and Engineering C 49 (2015) 640-647.

[106] X. Luo, A. Morrin, A.J. Killard, M.R. Smyth. Application of nanoparticles in electrochemical sensors and biosensors. Electroanalysis 18 (2006) 319-326.

[107] J.M. George, A. Antony, B. Mathew. Metal oxide nanoparticles in electrochemical sensing and biosensing: a review. Microchimica Acta 185 (2018) 1-26. 
[108] F.W. Campbell, R.G. Compton. The use of nanoparticles in electroanalysis: an updated review. Analytical and Bioanalytical Chemistry 396 (2010) 241-259.

[109] K. Saha, S.S. Agasti, C. Kim, X. Li, V.M. Rotello. Gold nanoparticles in chemical and biological sensing. Chemical Reviews 112 (2012) 2739-2779.

[110] F. Wang, S. Hu. Electrochemical sensors based on metal and semiconductor nanoparticles. Microchimica Acta 165 (2009) 1-22.

[111] E. Katz, I. Willner, J. Wang. Electroanalytical and bioelectroanalytical systems based on metal and semiconductor nanoparticles. Electroanalysis 16 (2004) 19-44.

[112] C. Medina-Plaza, C. García-Cabezón, C. García-Hernández, C. Bramorski, Y. Blanco-Val, F. Martín-Pedrosa, T. Kawai, J.A. De Saja, M.L. Rodríguez-Méndez. Analysis of organic acids and phenols of interest in the wine industry using Langmuir-Blodgett films based on functionalized nanoparticles. Analytica Chimica Acta 853 (2015) 572-578.

[113] T. Alizadeh, S. Mirzagholipur. A nafion-free non-enzymatic amperometric glucose sensor based on copper oxide nanoparticles - graphene nanocomposite. Sensors and Actuators B 198 (2014) 438-447.

[114] M. Fouladgar, H. Karimi-Maleh, V. Gupta. Highly sensitive voltammetric sensor based on NiO nanoparticle room temperature ionic liquid modified carbon paste electrode for levodopa analysis. Journal of Molecular Liquids 208 (2015) 78-83.

[115] W.S. Alencar, F.N. Crespilho, M.V.A. Martins, V. Zucolotto, O.N. Oliveira, W.C. Silva. Synergistic interaction between gold nanoparticles and nickel phthalocyanine in layer-by-layer (LbL) films: evidence of constitutional dynamic chemistry (CDC). Physical Chemistry Chemical Physics 11 (2009) 5086-5091.

[116] M. Pal, V. Ganesan. Zinc phthalocyanine and silver/gold nanoparticles incorporated MCM-41 type materials as electrode modifiers. Langmuir 25 (2009) 13264-13272.

[117] C. Hou, H. Liu, D. Zhang, C. Yang, M. Zhang. Synthesis of ZnO nanorods- Au nanoparticles hybrids via in-situ plasma sputtering-assisted method for simultaneous electrochemical sensing of ascorbic acid and uric acid. Journal of Alloys and Compounds 666 (2016) 178-184.

[118] D. Zhai, B. Liu, Y. Shi, L. Pan, Y. Wang, W. Li, R. Zhang, G. Yu. Highly sensitive glucose sensor based on Pt nanoparticle/polyaniline hydrogel heterostructures. ACS Nano 7 (2013) 3540-3546.

[119] R. Sandulescu, M.Tertis, C. Cristea, E. Bodoki. New materials for the construction of electrochemical biosensors in Biosensors - Micro and Nanoscale Applications. T. Rinken (Ed.). IntechOpen: London, 2015.

[120] J. Zeravik, A. Hlavacek, K. Lacina, P. Skládal. State of the art in the field of electronic and bioelectronic tongues - Towards the analysis of wines. Electroanalysis 21 (2009), 2509-2520.

[121] K. Toko. Biochemical sensors: Mimicking gustatory and olfactory senses. Jenny Standford Publishing: New York, 2013.

[122] C. Medina-Plaza, J.A. de Saja, M.L. Rodriguez-Mendez. Bioelectronic tongue based on lipidic nanostructured layers containing phenol oxidases and lutetium bisphthalocyanine for the analysis of grapes. Biosensors and Bioelectronics 57 (2014) 276-286.

[123] J. Zhou, X. Li, L.L. Yang, S.L. Yan, M.M. Wang, D. Cheng, Q. Chen, Y.L. Dong, P. Liu, W.Q. Cai, C.C. Zhang. The Cu-MOF-199/single-walled carbon nanotubes modified electrode for simultaneous determination of hydroquinone and catechol with extended linear ranges and lower detection limits. Analytica Chimica Acta 899 (2015) 57-65

[124] I.M. Apetrei, C. Apetrei. The biocomposite screen-printed biosensor based on immobilization of tyrosinase onto the carboxyl functionalised carbon nanotube for assaying tyramine in fish products. Journal of Food Engineering 149 (2015) 1-8.

[125] I.M. Apetrei, C. Apetrei. Amperometric biosensor based on polypyrrole and tyrosinase for the detection of tyramine in food samples. Sensors and Actuators B 178 (2013) 40-46.

[126] C. Apetrei, M.L. Rodríguez-Méndez, J.A. De Saja. Amperometric tyrosinase based biosensor using an electropolymerized phosphate-doped polypyrrole film as an immobilization support. Application for detection of phenolic compounds. Electrochimica Acta 56 (2011) 8919-8925.

[127] K. Wang, L.N. Dai, Q. Liu, H.N. Li, C. Ju, J. Wu, H.M. Li, H.M. (2011). Electrodeposition of unsubstituted iron phthalocyanine nano-structure film in a functionalized ionic liquid and its electrocatalytic and electroanalysis applications. Analyst 136 (2011) 4344-4349. 
[128] I. Ponce, J.F. Silva, R. Oñate, M.C. Rezende, M.A. Paez, J. Pavez, J.H. Zagal. Enhanced catalytic activity of Fe phthalocyanines linked to $\mathrm{Au}(111)$ via conjugated self-assembled monolayers of aromatic thiols for $\mathrm{O} 2$ reduction. Electrochemistry Communications 13 (2011) 1182-1186.

[129] J.B. Han, X.Y. Xu, X.Y. Rao, M. Wei, D.G. Evans, X.J. Duan. Layer-by-layer assembly of layered double hydroxide/cobalt phthalocyanine ultrathin film and its application for sensors. Journal of Materials Chemistry 21 (2011) 2126-2130

[130] P.H.B. Aoki, D. Volpati, A. Riul Jr., W. Caetano, C.J.L. Constantino. Layer-by-Layer technique as a new approach to produce nanostructured films containing phospholipids as transducers in sensing applications. Langmuir 25 (2009) 2331-2338.

[131] D. Volpati, P. Alessio, A.A. Zanfolim, F.C. Storti, A.E. Job, M. Ferreira, A. Riul, O.N. Oliveira, C.J.L. Constantino. Exploiting distinct molecular architectures of ultrathin films made with iron phthalocyanine for sensing. Journal of Physical Chemistry B 112 (2008) 15275-15282.

[132] A.C. Santos, V. Zucolotto, C.J.L. Constantino, H.N. Cunha, J.R. dos Santos Jr., C. Eiras. Electroactive LBL films of metallic-phthalocyanines and poly (o-methoxyaniline) for sensing. Journal of Solid State Electrochemistry 11 (2007) 1505-1510

[133] C. Medina-Plaza, L.N. Furini, C.J.L. Constantino, J.A. de Saja, M.L. Rodriguez-Mendez. Synergistic electrocatalytic effect of nanostructured mixed films formed by functionalised gold nanoparticles and bisphthalocyanines. Analytica Chimica Acta 851 (2014) 95-102.

[134] L.N. Furini, E. Feitosa, P. Alessio, M.H. Shimabukuro, A.Jr. Riul, C.J.L. Constantino. Tuning the nanostructure of DODAB/nickel tetrasulfonated phthalocyanine bilayers in LbL films. Materials Science and Engineering C 33 (2013) 2937-2946

[135] M. Ferreira, V. Zucolotto, O.N. Oliveira Jr., K. Wohnrath. Encyclopedia of nanoscience and nanotechnology 4. H.S. Nalwa (Ed.). American Scientific Publishers: Los Angeles, 2003. pp. 441-460.

[136] R. Ahmad, O.S. Wolfbeis, Y.B. Hahn, H.N. Alshareef, L. Torsi, K.N. Salama. Deposition of nanomaterials: A crucial step in biosensor fabrication. Materials Today Communications 17 (2018) 289-321.

[137] K. Ariga, T. Nakanishi, T. Michinobu. Immobilization of biomaterials to nano-assembled films (Self-Assembled Monolayers, Langmuir-Blodgett Films, and Layer-by-Layer Assemblies) and their related functions. Journal of Nanoscience and Nanotechnology 6 (2006) 2278-2301.

[138] C. Apetrei, P. Alessio, C.J.L. Constantino, J.A. De Saja, M.L. Rodriguez-Mendez, F. Pavinatto, E.G.R. Fernandes, V. Zucolotto, O.N. Oliveira. Biomimetic biosensor based on lipidic layers containing tyrosinase and lutetium bisphthalocyanine for the detection of antioxidants. Biosensors and Bioelectronics 26 (2011) 2513-2519.

[139] P.H.B. Aoki, P. Alessio, D. Volpati, F.V. Paulovich, A. Riul Jr., O.N. Oliveira Jr., C.J.L. Constantino. On the distinct molecular architectures of dipping- and spray-LbL films containing lipid vesicles. Materials Science and Engineering C 41 (2014) 363-371

[140] P. Alessio, C.S. Martin, J.A. de Saja, M.L. Rodriguez-Mendez. Mimetic biosensors composed by layer-by-layer films of phospholipid, phthalocyanine and silver nanoparticles to polyphenol detection. Sensors and Actuators B 233 (2016) 654-666.

[141] F.J. Pavinatto, E.G.R. Fernandes, P. Alessio, C.J.L. Constantino, J. A. de Saja, V. Zucolotto, C. Apetrei, O.N. Oliveira Jr., M.L. Rodriguez-Mendez. Optimized architecture for Tyrosinase-containing Langmuir-Blodgett films to detect pyrogallol. Journal of Materials Chemistry 21 (2011) 4995-5003.

[142] K. Woertz, C. Tissen, P. Kleinebudde, J. Breitkreutz. Performance qualification of an electronic tongue based on ICH guideline Q2. Journal of Pharmaceutical and Biomedical Analysis 51 (2010) 497-506.

[143] M. Pein, D. Kirsanov, P. Ciosek, M. del Valle, I. Yaroshenko, M. Wesoły, M. Zabadaj, A. Gonzalez-Calabuig, W. Wróblewski, A. Legin. Independent comparison study of six different electronic tongues applied for pharmaceutical analysis. Journal of Pharmaceutical and Biomedical Analysis 114 (2015) 321-329.

[144] L. Lvova, E. Martinelli, F. Dini, A. Bergamini, R. Paolesse, C. Di Natale, A. D’Amico. Clinical analysis of human urine by means of potentiometric electronic tongue. Talanta 77 (2009) 1097-1104.

[145] I. Yaroshenko, D. Kirsanov, L. Kartsova, A. Sidorova, I. Borisova, A. Legin. 2015. Determination of urine ionic composition with potentiometric multisensor system. Talanta 131 (2015) 556-561.

[146] J.E. Oliveira, V. Grassi, V.P. Scagion, L.H.C. Mattoso, G.M. Glenn, E.S. Medeiros. Sensor array for water analysis based on interdigitated electrodes modified with fiber films of poly(lactic acid)/multiwalled carbon nanotubes. IEEE Sensors Journal 13 (2013) 759-766. 
[147] O. Zadorozhnaya, D. Kirsanov, I. Buzhinsky, F. Tsarev, N. Abramova, A. Bratov, F.J. Muñoz, J. Ribó, J. Bori, M.C. Riva, A. Legin. Water pollution monitoring by an artificial sensory system performing in terms of Vibrio fischeri bacteria. Sensors and Actuators B 207 (2015) 1069-1075.

[148] A. Fujita, A. Isogai, M. Endo, H. Utsunomiya, S. Nakano, H. Iwata. Effects of sulfur dioxide on formation of fishy off-odor and undesirable taste in wine consumed with seafood. Journal of Agricultural and Food Chemistry 58 (2010) 4414-4420.

[149] T. Ujihara, N. Hayashi, H. Ikezaki, H. Objective evaluation of astringent and umami taste intensities of Matcha using a taste sensor system. Food Science and Technology Research 19 (2013) 1099-1105.

[150] H. Akitomi, Y. Tahara, M. Yasuura, Y. Kobayashi, H. Ikezaki, K. Toko. Quantification of tastes of amino acids using taste sensors. Sensors and Actuators B 179 (2013) 276-281.

[151] Y. Kobayashi, M. Habara, H. Ikezazki, R. Chen, Y. Naito, K. Toko. Advanced taste sensors based on artificial lipids with global selectivity to basic taste qualities and high correlation to sensory scores. Sensors 10 (2010) 34113443.

[152] K. Woertz, C. Tissen, P. Kleinebudde, J. Breitkreutz. A comparative study on two electronic tongues for pharmaceutical formulation development. Journal of Pharmaceutical and Biomedical Analysis 55 (2011) 272-281.

[153] X. Tian, J. Wang, X. Zhang. Discrimination of preserved licorice apricot using electronic tongue. Mathematical and Computer Modelling 58 (2013) 737-745.

[154] S. Xu, J. Li, E.A. Baldwin, A. Plotto, E. Rosskopf, J.C. Hong, J. Bai. Electronic tongue discrimination of four tomato cultivars harvested at six maturities and exposed to blanching and refrigeration treatments. Postharvest Biology and Technology 136 (2018) 42-49.

[155] H.Y. Jung, H.S. Kwak, M.J. Kim, Y. Kim, K.O. Kim, S.S. Kim. Comparison of a descriptive analysis and instrumental measurements (electronic nose and electronic tongue) for the sensory profiling of Korean fermented soybean paste (doenjang). Journal of Sensory Studies 32 (2017) 1-11.

[156] Y. Shen, P. Chen, W.J. Chen, H.M. Chen. Haiming. Monitoring the quality change of fresh Coconut milk using an electronic tongue. Journal of Food Processing and Preservation 41 (2017) 1-7.

[157] P. Ciosek, W. Wróblewski. Sensor arrays for liquid sensing-Electronic tongue systems. Analyst 132 (2007) 963-978.

[158] A. Zakaria, A.Y.M. Shakaff, M.J. Masnan, M.N. Ahmad, A.H. Adom, M.N. Jaafar, S.A. Ghani, A.H. Abdullah, A.H.A. Aziz, L.M. Kamarudin, N. Subari, N.A. Fikri. A biomimetic sensor for the classification of honeys of different floral origin and the detection of adulteration. Sensors 11 (2011) 7799-7822.

[159] M.L. Rodríguez-Méndez, J.A. de Saja, C. Medina-Plaza, C. García- Hernández. Electronic tongues for the organoleptic characterization of wines. In Electronic Noses and Tongues in Food Science, M.L. Rodríguez-Méndez (Ed.). Elsevier: Amsterdam, 2016, pp. 265-273.

[160] N. Prieto, M. Gay, S. Vidal, O. Aagaards, J.A. de Saja, M.L., Rodriguez-Mendez. Analysis of the influence of the type of closure in the organoleptic characteristics of a red wine by using an electronic panel. Food Chemistry 129 (2011) 589-594.

[161] A. Rudnitskaya, S.M. Rocha, A. Legin, V. Pereira, J.C. Marques. Evaluation of the feasibility of the electronic tongue as a rapid analytical tool for wine age prediction and quantification of the organic acids and phenolic compounds. The case-study of Madeira wine. Analytica Chimica Acta 662 (2010) 82-89.

[162] V. Parra, A. Arrieta, J.A. Fernández-Escudero, M. Iñiguez, J.A. de Saja, M.L. Rodríguez-Méndez. Monitoring of the ageing of red wines in oak barrels by means of a hybrid electronic tongue. Analytica Chimica Acta 563 (2006) 229-237.

[163] X. Cetó, J. Capdevilla, S. Minguez, M. del Valle. Voltammetric bioelectronic tongue for the analysis of phenolic compounds in rosé cava wines. Food Research International 55 (2014) 455-461.

[164] L. Gil-Sanchez, J. Soto, R. Martinez-Mañez, E. Garcia-Breijo, J. Ibañez, E. Llobet. A novel humid electronic nose combined with an electronic tongue for assessing deterioration of wine. Sensors and Actuators A 171 (2011) 152-158.

[165] G. Verrelli, L. Francioso, R. Paolesse, P. Siciliano, C. Di Natale, A. D’Amico, A. Logrieco. Development of silicon-based potentiometric sensors: Towards a miniaturized electronic tongue. Sensors and Actuators B 123 (2007) 191-197. 
Doctoral Thesis: Electronic tongue technology applied to the analysis of grapes and wines

\section{RESULTS AND DISCUSSION}





\subsection{Introduction}

In this chapter, a selected number of papers published in international journals as a result of the research work carried out during the doctoral studies in the group UVaSens from the University of Valladolid are presented. The papers appear as follows:

Paper I: Multisensor systems based on phthalocyanines for monitoring the quality of grapes.

Paper II: Monitoring the phenolic ripening of red grapes using a multisensor system based on metal-oxide nanoparticles.

Paper III: A different approach for the analysis of grapes: Using the skin as sensing element.

Paper IV: Electrochemical behavior of polypyrrol/AuNP composites deposited by different electrochemical methods: sensing properties towards catechol.

Paper V: Analysis of musts and wines by means of a bio-electronic tongue based on tyrosinase and glucose oxidase using polypyrrole/gold nanoparticles as the electron mediator.

Paper VI: Layered composites of PEDOT/PSS/nanoparticles and PEDOT/PSS/phthalocyanines as electron mediators for sensors and biosensors.

Paper VII: Impedimetric electronic tongue based on nanocomposites for the analysis of red wines. Improving the variable selection method.

Paper VIII: An electrochemical quartz crystal microbalance multisensor system based on phthalocyanine nanostructured films: Discrimination of musts.

Paper IX: In situ synthesis, stabilization and activity of protein-modified gold nanoparticles for biological applications.

UVaSens is an expert multidisciplinary research group in the development of electrochemical sensors, mainly voltammetric, for their application in electronic tongues. Its research work includes the manufacture and characterization of classical sensors based on CPE, spin-coating or casting, but also the preparation of nanostructured thin films using Layer by Layer or Langmuir-Blodgett techniques. In addition, for the construction of the sensors, the group has widely used sensing electrocatalytic materials, such as phthalocyanines and bisphthalocyanines, in both nanostructured and non-nanostructured sensors. The group has also worked with other 
interesting materials in the manufacture of sensors, such as conducting polymers, when they began to be applied in electrochemical sensors. The UVaSens group was one of the first to develop modified voltammetric electronic tongues based on CPE and phthalocyanines to improve the electrochemical responses. Thus, paper I, "Multisensor systems based on phthalocyanines for monitoring the quality of grapes", is a review article that gives a general overview of electronic tongues based on phthalocyanines for the analysis of grapes in which the use of non-nanostructured and nanostructured sensors and biosensors is discussed.

Research papers II to VII are based on electrochemical sensors prepared by classical techniques, such as CPE, electrodeposition and spin-coating; whereas, in papers VIII and IX, we have applied nanoscience and nanotechnology, concretely, the LbL technology.

In papers II and III, we have used sensors based on CPE which are easy to prepare and are low cost. In paper II, "Monitoring the phenolic ripening of red grapes using a multisensor system based on metal-oxide nanoparticles", graphite was mixed with different metal oxide nanoparticles to construct an array of sensors. This array, containing metal oxide nanoparticles, has been used as a voltammetric electronic tongue for the first time and was applied to monitoring grape ripeness according to their phenolic maturity. In addition, the voltammetric responses obtained with this electronic tongue have shown good correlations with the phenolic content parameter of the grapes. Moreover, these sensors have the advantage that they can easily be recovered by peeling the surface with a filter paper, which makes them highly appropriate for industrial applications.

In paper III, "A different approach for the analysis of grapes: Using the skin as sensing element", we have developed an original work never proposed before, in which the sensing material is the grape skin itself. Thus, the skin was deposited onto a CPE electrode with an intimate contact between the graphite and the inner side of the skin. Considering that grape skin contains phenols that are released to the pulp during the ripening process and that these influence the color, sensory sensations and quality of both the grapes and future wines, by analysing the skin, we can study grape ripeness directly. However, some issues must be improved and taken into account in order to get better correlations between the sensor responses and the chemical parameters obtained by traditional methods.

Papers IV to VII are based on sensors modified with well-known conducting polymers. In paper IV, "Electrochemical behavior of polypyrrol/AuNP composites deposited by different electrochemical methods: sensing properties towards catechol", we have electrodeposited 
a film of polypyrrole onto platinum and stainless steel substrates and also incorporated AuNPs in the polymeric films to enhance the electrocatalytic performance of the electrochemical sensors. Sensors as-prepared were characterized by using SEM, EIS and cyclic voltammetry. Then, they were applied to the analysis of the polyphenol catechol, which is a phenolic group of interest in the oxidation process in wine. These sensors were used later in paper $\mathrm{V}$, "Analysis of musts and wines by means of a bio-electronic tongue based on tyrosinase and glucose oxidase using polypyrrole/gold nanoparticles as the electron mediator", to develop a voltammetric electronic tongue for the first time to analyse must and the subsequent wines. Furthermore, in this work, we used two enzymes, tyrosinase and glucose oxidase, to improve the specificity of the sensors to phenols and sugars. The enzymes were deposited onto the electrode surface by means of the drop-casting technique. The novelty of this work lies in the fact that, never before, have the quality parameters of interest in wines been predicted from the previously obtained voltammetric data in musts. Therefore, oenologists could know the quality of their wines from the beginning of the vinification process.

Papers VI and VII are based on the highly electrical conducting polymer PEDOT:PSS. Thus, in paper VI, "Layered composites of PEDOT/PSS/nanoparticles and PEDOT/PSS/phthalocyanines as electron mediators for sensors and biosensors", we have used the spin-coating technique to prepare ITO substrates modified with a polymer layer of PEDOT:PSS and we have also incorporated a secondary layer of electrocatalytic material, such as AuNP, copper phthalocyanine and lutetium bisphthalocyanine to improve the electrochemical performance of the sensors. In addition, the enzymes tyrosinase and laccase were deposited onto the electrode surface by drop-casting to improve the specificity. These sensors and biosensors have shown excellent limits of detection and reproducibility for catechol and hydroquinone. After these excellent results, in paper VII, "Impedimetric electronic tongue based on nanocomposites for the analysis of red wines. Improving the variable selection method", we decided to apply electrochemical sensors based on PEDOT:PSS in an impedimetric electronic tongue for the authentication of wines elaborated with different varieties of grape and from different Denominations of Origin. This application is quite novel, since only one work has reported the use of an impedimetric tongue to analyze red wines and, in general, the use of impedimetric electronic tongues for food authenticity is rather scarce. Moreover, in this work, we used different strategies to find the best variables for further chemometric analysis, including the classical method of measuring resistance and capacitance data at a fixed 
frequency, selecting parameters from the electrochemical impedance spectroscope analysis, and using the parameters from an equivalent electrical circuit model.

Finally, papers VIII and IX are based on the LbL technology. In paper VIII, "An electrochemical quartz crystal microbalance multisensor system based on phthalocyanine nanostructured films: Discrimination of musts", nanostructured LbL films based on phtahlocyanines were deposited onto QCM substrates to be used as voltammetric EQCM sensors. This electronic tongue was used for the first time in the food industry recording voltammetric data and mass changes simultaneously. Musts of grapes of different varieties were analyzed and discriminated according to their sugar and phenolic content. In the field of food, a piezoelectric immunosensor based on QCM has been developed to determine pesticide residues and metabolites in fruit juices; however, most applications for QCM sensors are in electronic noses for the analysis of volatile organic compounds (VOCs), as piezoelectric immunosensors for the recognition of biomolecular interactions and as gravimetric sensors in several electrochemical studies (EQCM). The main advantage of EQCM sensors over traditional electrochemical sensors is that they provide more information (mass and electrochemical) in a single experiment. Moreover, in comparison with the works mentioned above, sensors based on LbL nanostructures are more sophisticated and provide a higher surface area to volume ratio, but are still easy to prepare and inexpensive.

In the last paper, "In situ synthesis, stabilization and activity of protein-modified gold nanoparticles for biological applications", the LbL technique has also been used although with a dissimilar purpose. In this case, LbL was applied to prepare a nanostructured carrier system based on AuNPs, where the enzyme lysozyme has been immobilized using stronger interactions. This work was developed at the Missouri State University during a short research stay of 4 months. Although this work is not based on the development of sensors to be applied in electronic tongues or even in the food industry, it can be extended to that field, since the reported nanostructured assembly of the enzyme can be performed for others of interest in the food industry, such as tyrosinase, laccase or GOx, which have already been used in the works mentioned above and with the possibility of creating many designs thanks to the versatility of the LbL technique. 


\subsection{Paper I}

Multisensor systems based on phthalocyanines for monitoring the quality of grapes.

By María Luz Rodríguez-Méndez, Celia García-Hernández, Cristina Medina-Plaza, Cristina García-Cabezón and José Antonio de Saja. Journal of Porphyrins and Phthalocyanines 20 (2016) 889-894. (doi: 10.1142/S1088424616500796) 


\subsubsection{Motivation and objective}

In last few years, the control of phenolic maturity in grapes has become more essential for quality control purposes. Traditionally, wine growers have estimated the optimal harvesting date by evaluating the sugar content and acidity of grapes, but other compounds like phenols are also as important as sugars and acids present in grapes, due to the fact that phenolic composition will affect the final organoleptic characteristics of the wines.

The use of quick, reliable and cheap sensors for monitoring the quality of grapes represents an important need in the wine sector. Electronic tongues are good candidates due to their advantages of higher sensitivity and relatively low cost in comparison with other methods like spectroscopy (FTIR, FT-NIR) or gas and liquid chromatography (GC, HPLC). Electronic tongues have been widely used in food quality control but, in the case of grapes and wines, there are many more research works dedicated to the analysis of wine than the number of works dedicated to the analysis of grapes or musts.

In addition, electrochemical sensors can be chemically modified with sensing materials by means of several techniques to improve their sensitivity and specificity. Among the variety of sensing materials that have been used in the modification of sensors, metallophthalocyanines (MPc) have been demonstrated to be a good choice, due to their excellent electrocatalytic properties. The catalytic activity of MPc for electrochemical reactions is due to their macrocyclic nature with extended $\pi$-systems (or conjugated systems) where electrons are delocalized about the system. In this way, MPc are capable of undergoing fast redox processes with minimal reorganizational energies and can act as electron mediators in transfer processes involving a great variety of molecules. There are many methods to incorporate MPcs to an electrode surface: the adsorption of the MPcs onto graphite or carbon materials, adsorption of monolayers of MPc by dipping the electrode into a solution containing the complexes, dropping the solution of the complexes onto the electrode surface, by self-assembled monolayers (SAMs) of thiol-derivatized phthalocyanines on gold substrates, by means of the electropolymerization of a functionalized monomeric phthalocyanine, by coating the electrode with a preformed polymer containing MPcs, or by using thin films techniques like Langmuir-Blodgett (LB) or Layer by Layer (LbL).

The modified electrode can be characterized by simple electrochemical techniques, such as cyclic voltammetry, which allows the estimation of the formal potentials of redox processes 
involving the immobilized MPc and also the amount of active species present on the electrode surface.

In this review, the state of the art of electronic tongues based on phthalocyanines applied in the analysis of the quality of grapes is described. Particular attention is paid to voltammetric sensors.

\subsubsection{Summary of the research work}

Carbon paste electrodes were used to prepare the simplest sensor array based on MPc to analyze grapes of different variety. Cobalt phthalocyanine, copper phthalocyanine, zinc phtahlocyanine and lutetium bis-phthalocyanine were used to modify the graphite electrodes. The sensors were prepared by mixing graphite, mineral oil (nujol) and MPc forming a homogeneous paste that was later placed in a plastic syringe. A copper wire was used as electrical contact. The voltammetric responses of the sensors showed anodic peaks at 0.4-0.8 $\mathrm{V}$ related to phenols present in musts and a cathodic peak at $-0.5 \mathrm{~V}$ that could be associated to glucose or protons. Lutetium bis-phthalocyanine and cobalt phthalocyanine CPEs evidenced a higher electrocatalytic effect, with intensities for catechol and glucose much higher than copper phthalocyanine and zinc phathalocyanine CPEs. In addition, the variety in the electrochemical responses of the different mettallophthalocyanines used provided a degree of cross-selectivity that allowed us to discriminate between grape varieties, by means of PCA, according to their sugar and polyphenolic content.

On the other hand, other alternatives to traditional electrodes are nanostructured thin films based sensors consisting in preparing homogeneous surfaces with an increased number of active sites, thus improving in the sensitivity of the electrochemical sensors. The analysis of different grape varieties was carried out using a multisensor system based on LbL films of iron, nickel and copper tetrasulfonic phthalocyanins. The LbL films were deposited onto quartz crystal microbalance sensors (QCM) in order to simultaneously register the electrochemical response and mass changes occurring during redox processes. This work allowed us to use these sensors as an electronic tongue based on EQCM sensors to discriminate between grapes with different sugar and phenolic contents. 


\section{Chapter 3: Results and discussion}

Paper I: Multisensor systems based on phthalocyanines for monitoring the quality of grapes.

Finally, going one step further, the specificity of electrochemical sensors can be improved by incorporating enzymes to develop biosensors. The immobilization of enzymes onto electrode surfaces is not easy to achieve. It is important to deposit the enzyme in a way that will still maintain the enzymatic activity. LbL or Langmuir-Blodgett (LB) techniques can be used to adsorb enzymes in lipidic membranes, creating a kind of biomimetic environment. Moreover, the electron transfer between the enzymes and the electrode surface can be improved through a mediator such as phthalocyanines. Grapes with different phenolic content were analyzed by means of a voltammetric electronic tongue based on nanostructured sensors, prepared by the LB technique and made up of one non-specific sensor based on a phthalocyanine and two biosensors based on arachidic acid, a phthalocyanine and the enzymes laccase and tyrosinase. These two enzymes are phenoloxidases that catalyze the oxidation-reduction of phenols and improve the specificity of the sensors for phenols present in the grapes. The voltammetric responses of the sensors for grapes of different varieties allow us to discriminate between the samples according to their phenolic content by using PCA and, moreover, using PLS-1, good correlations with the chemical parameters of total polyphenol Index, acidity and pH (obtained by traditional methods) were found, showing high correlation coefficients and low errors for a small number of latent variables. 


\title{
Multisensor systems based on phthalocyanines for monitoring the quality of grapes
}

\author{
Maria Luz Rodriguez-Mendez ${ }^{\star a}$, Celia García-Hernandez ${ }^{a}$, Cristina Medina-Plaza ${ }^{a}$, \\ Cristina García-Cabezón ${ }^{\mathrm{b}}$ and Jose Antonio de Saja ${ }^{\mathrm{c}}$
}

a Department of Inorganic Chemistry, Escuela de Ingenierías Industriales, Universidad de Valladolid, Paseo del Cauce 59, 47011 Valladolid, Spain

${ }^{b}$ Department of Materials Science, Escuela Ingenierías Industriales, Universidad de Valladolid, 47011 Valladolid, Spain

${ }^{\circ}$ Department of Condensed Matter Physics, Universidad de Valladolid, 47011 Valladolid, Spain

Dedicated to Professor Tomás Torres on the occasion of his 65th birthday

Received 24 February 2016

Accepted 9 March 2016

\begin{abstract}
Arrays of phthalocyanine-based sensors with complementary activity have been used to develop voltammetric electronic tongues. Such systems have demonstrated to be useful in enology for the evaluation of quality of wines in different production stages, from grapes to bottles. In this paper, the state of the art of multisensor systems based on phthalocyanines dedicated to the analysis of musts (juices obtained from crushed grapes) is described. Such multisensor systems cover different types of sensors from simple Carbon Paste Electrodes, to sophiticated nanostructured sensors, including Langmuir-Blodgett or Layer by Layer thin films and biomimetic biosensors where phthalocyanines play a crucial role as electron mediator between enzymes and electrodes. In all cases, multisensor systems based on phthalocyanines have been able to discriminate musts prepared from different varieties of grapes. The performance of these systems can be improved by combining non-specific sensors with biosensors containing enzymes selective to phenols. In this case, excellent relationships have been found between the responses provided by the array and the content in phenols and acids provided by traditional chemical analysis.
\end{abstract}

KEYWORDS: electronic tongue, phthalocyanines, voltammetric sensors, wines, grapes.

\section{INTRODUCTION}

Phthalocyanines have been extensively used as sensing materials due to their physicochemical properties [1-8]. A variety of sensors based on phthalocyanines has been developed using different strategies that include resistive, chemical, optical or mass transduction [9-11]. Phthalocyanines are particularly useful in the field of electrochemical sensors (potentiometric, amperometric, voltamperometric or electrochemical impedance) [9-13]. From the large family of phthalocyanine molecules, metalophthalocyanines (MPc) have been the most widely

*Correspondence to: María Luz Rodríguez-Méndez, email: mluz@eii.uva.es, tel: +34 983 423540; fax: +34983423310 studied derivatives. Double decker derivatives $\left(\mathrm{LnPc}_{2}\right)$ have also attracted considerable interest as voltammetric sensors [9, 12-14].

Voltammetric sensors modified with phthalocyanines are commonly used to analyze compounds with redox activity. The number of papers published in this field is quite large and include a variety of phthalocyanine compounds, electrode designs, and target molecules [9-14]. Many papers have been dedicated to the analysis of compounds of interest in the food industry such as antioxidants, sugars and organic acids among many others, because the electrocatalytic properties of electrodes chemically modified with phthalocyanines decrease in a large extent the oxidation potential of these natural reducing agents [15-19]. Phthalocyanines have 
also obtained great success in the field of multisensor systems, the so-called electronic tongues (ET). According to the IUPAC, an ET is a multisensor system formed by a number of non-selective sensors combined with multivariate chemometric tools [20]. ETs try to mimic the biological taste systems which contain sensors that non-selectively interact with flavor molecules to produce electronic signals. Such signals are processed using pattern recognition software. Using non-supervised methods such as Principal Component Analysis (PCA) samples with different characteristics can be discriminated. Using supervised techniques such as Artificial Neural Networks (ANN) it is possible to classify samples belonging to different classes. The produced signals can also be correlated to the composition of the studied solution. ETs are used to analyze complex mixtures and provide global information about the sample instead of giving information of particular constituents. Due to this holistic approach, ET are particularly interesting for the analysis of complex liquids such as drinks and beverages [18-24].

Arrays of phthalocyanine-based sensors with complementary activity have been used in voltammetric electronic tongues dedicated to the analysis of saffron [25], oils [26, 27], beers [28], milk [29] and wines [24, 30-32]. The complexity of these liquids makes them ideal samples to be analyzed with multisensor systems that will give global information of the sample as the human senses do.

The analysis of grapes is becoming more and more important because the final quality of wine is directly related to the quality of grapes. In the vineyard, the quality of grapes is usually established by measuring their sugar content and acidity. Nowadays, it is becoming evident that the quality of grapes is also closely related to many other parameters such as their phenolic composition [33, 34]. This makes necessary to develop new methods to assess the quality or the ripeness state. ETs could be able to provide the necessary complementary information needed to decide important issues such as the retail price (marked by the quality) or the appropriate date for harvesting.

During the last few years our group has been involved in the design of ETs. In this paper, the state of the art of ETs based on phthalocyanines devoted to the analysis of grapes will be presented. Examples wil be given to illustrate how multisensor systems based on phthalocyanines can be used to analyze the quality of grapes efficiently. The examples of ETs will include from arrays formed by simple and cheap electrodes to sophisticated nanostructured sensors and biosensors.

\section{ELECTRONIC TONGUES BASED ON PHTHALOCYANINES FOR THE ANALYSIS OF GRAPES}

Electronic tongues have been widely used to analyze wines, however, the number of works dedicated to the analysis of grapes or musts (the juice obtained after pressing grapes) is quite small. ETs can use conventional ISFETS [35], but most work use voltammetric sensors. Voltammetric electrodes chemically modified with phthalocyanines are particularly suitable to discriminate grapes [36]. When phthalocyanine based electrodes are immersed in a must which is a complex solution containing ions $\mathrm{Na}^{+}, \mathrm{K}^{+}, \mathrm{H}^{+}$, etc.) and redox active species (antioxidants, sugars, etc.), voltammograms show peaks of two different origins: peaks associated with the oxidation-reduction of the redox species present in the solution and the transient responses associated with the electrode material. In addition, the interactions between the electrode and the solution can improve the selectivity of the electrodes. These interactions include: (i) antioxidants present in musts can modify the oxidation potential of the phthalocyanine deposited at the electrode surface; (ii) the electrocatalytic activity of the phthalocyanine can facilitate the oxidation of the redox species present in must; (iii) the response of the electrode material is related to the ability of ions to diffuse inside the phthalocyanine layer, an influx necessary to preserve the macroscopic electroneutrality of the electrode; and (iv) changes in $\mathrm{pH}$ can produce modifications in the redox potentials of both the phthalocyanine (due to protonation) or the solution (changes in the redox potential of phenols). All these redox processes and

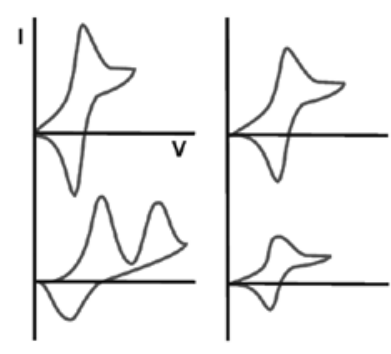

(a)
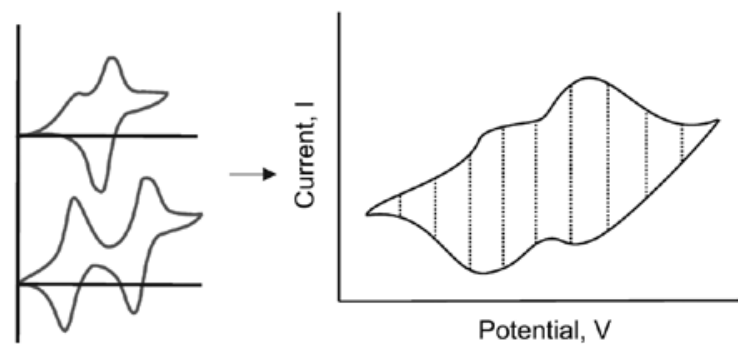

(b)

Fig. 1. Scheme of the principles of a voltammetric electronic tongue. (a) Variety of voltammetric signals from an array of sensors and (b) Pre-treatment of a voltammetric signal which is divided into 10 parts to obtain 10 variables 
interactions give rise to rich voltammograms that differ from one phthalocyanine to another and from one must to another, increasing the degree of selectivity (Fig. 1a) [24]. The whole voltammogram can be used as the output of each sensor device (instead a single peak as other electrochemicals sensors do). In consequence, a pretreatment step to reduce the number of variables (without loss of information) is required. Using the Kernel method, voltammograms are multiplied by a 10 smooth, bell-shaped windowing functions, and integrated with respect to potential. The idea behind this pre-processing technique is to capture the information throughout the global response to obtain 10 parameters per curve (Fig. 1b) $[30,37]$. Once the voltammograms have been pre-processed and the number of variables reduced, such variables are used as the input for statistical analysis using for instance Principal Component Analysis, Parallel Factor Analysis (PARAFAC) or Partial Least Squares Discriminant Analysis (PLS-DA) as discrimination and classification tools.

Besides their electrocatalytic properties, another important advantage of phthalocyanines is that they can be deposited onto electrode surfaces using a variety of techniques from simple and cheap procedures to advanced methods that produce nanostructured electrodes with enhanced performances [16, 17].

The next sections will describe ETs based on different types of voltammetric sensors based on phthalocyanines dedicated to the analysis of grapes.

\section{Electronic tongue based on carbon paste electrodes (CPE)}

Sensors based on phthalocyanines can be prepared using the carbon paste technique by mixing graphite powder and the corresponding phthalocyanine $(15 \%$, $\mathrm{w} / \mathrm{w}$ ). The paste is formed using a binder such as Nujol. Pastes are then packed into the body of a plastic tube and compressed. A metallic copper wire is used as a contact. Carbon Paste Electrodes (CPE) thus prepared are ideal for industrial applications because they are cheap and can be easily recovered by cleaning the surface with a filter paper.

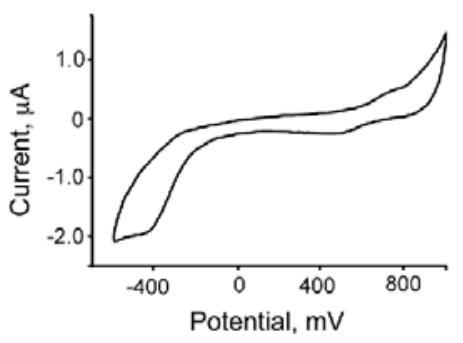

(a)
An array was formed by combining five $\mathrm{CPE}$ electrodes including $\mathrm{CoPc}(\mathrm{CoPc}-\mathrm{CPE}), \mathrm{CuPc}(\mathrm{CuPc}-\mathrm{CPE}), \mathrm{ZnPc}$ ( $\mathrm{ZnPc}-\mathrm{CPE})$, one electrode modified with $\mathrm{LuPc}_{2}\left(\mathrm{LuPc}_{2}-\right.$ $\mathrm{CPE})$ and one unmodified carbon paste electrode (C$\mathrm{CPE})[38,39]$. The system was used to analyze grapes of five different varieties (Tempranillo, Garnacha, Cabernet, Prieto Picudo and Mencía) harvested in 2012 in the D.O. Ribera de Duero region (Spain).

The electrochemical responses towards musts were dominated by the redox response of the phenolic compounds present in musts that appear as anodic peaks in the $0.4-0.8 \mathrm{~V}$ region. A cathodic peak in the $-0.5 \mathrm{~V}$ region was also observed that could be associated with the glucose but also with the presence of protons. In Fig. 2, the voltammetric response of the CPE electrodes is illustrated for a $\mathrm{CuPc}$ electrode immersed in a must obtained from Mencía variety. The important degree of cross-selectivity of the MPc-CPE array was related to the different electrocatalytic properties of the phthalocyanines used. For instance, the voltammetric responses obtained with $\mathrm{CoPc}$ and $\mathrm{LuPc}_{2}$ sensors towards catechol and glucose showed higher intensities than the obtained with $\mathrm{ZnPc}$ and $\mathrm{CuPc}$ sensors, confirming their superior electrocatalytic behavior.

Since voltammetric responses were related to the content of polyphenols, voltammograms could be used to discriminate must samples according to their composition. Using Principal Component Analysis (PCA) it was demonstrated that the array could be used to discriminate grapes. Clusters were located according to the composition of the musts in terms of polyphenolic content and sugar concentration, two of the main components responsible for their quality (Fig. 2b).

\section{Arrays of sensors based on Layer by Layer (LbL) nanostructured films}

The roughness of the sensors surface and the number of active sites depend on the technique used to prepare the electrodes, and these characteristics have a direct effect on the intensity of the responses. Nanostructured thin films show highly homogeneous surfaces with an increased number of active sites, improving the sensitivity

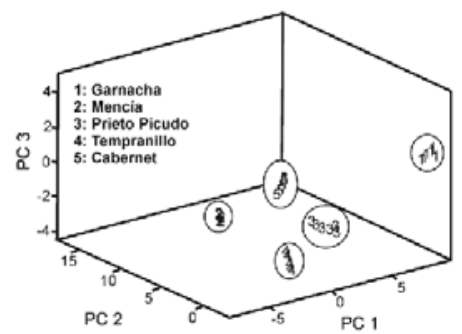

(b)

Fig. 2. (a) CuPc -CPE immersed in must prepared from grapes of the variety Mencia (diluted in water 1:2). (b) PCA obtained from the five musts analyzed 


\section{L. RODRIGUEZ-MENDEZ ET AL.}

of chemical sensors and facilitating the adsorptiondesorption processes of the analyzed molecules [40].

Typical methods used to fabricate nanostructured phthalocyanine films include electropolymerization [18], Layer by Layer (LbL) [41] and Langmuir-Blodgett technique (LB) [42].

The analysis of grapes was attempted with an array of voltammetric sensors formed by nanostructured LbL films of iron, nickel and copper tetrasulfonic phthalocyanines by alternating layers of the corresponding phthalocyanine with layers of poly-allylamin hydrochloride [36].

The secondary objective of this work was to combine electrochemical and mass measurements to study the mass changes accompanying the oxidation of electroactive species present in the sample. In fact, in previous works it was established that Quartz Crystal Microbalances (QCM) sensors covered with phthalocyanines could detect the presence of certain gases due to the mass changes that occur when gases are adsorbed on the phthalocyanine coated QCM sensors [43].

In our case, LbL films were deposited on Pt/QCM substrates through the Layer by Layer technique. The response of the array of sensors towards the must samples was studied by recording cyclic voltammetry and mass changes in synchronicity (Fig. 3). The combination of electrochemical and mass measurements (the so-called Electrochemical Quartz Crystal Microbalance, EQCM) was a new class of array of sensors that provided further information about the must samples.

Voltammograms registered by immersing the array in grape juices of six Spanish varieties of grapes (Juan García, Prieto Picudo, Mencía Regadío, Cabernet Sauvignon, Garnacha and Tempranillo) showed the characteristic features already described using CPE sensors. The peak positions associated with the phenolic content shifted to higher values when advancing in the transition metal series. In addition, because the polyphenolic content varied from one must to another, the positions, broadness and intensities of the peaks were different. The massograms registered simultaneously to the cyclic voltammetry are illustrated in Fig. 3. All massograms showed a progressive increase in mass, but also in this case, each must produced a different signature. The change in mass could be attributed to the adhesion of sugars and/or to other complex processes and polymerizations.

The mass and voltammetric outputs processed using PARAFAC demonstrated the capability of both types of signals to discriminate grapes according to their chemical composition: voltammograms discriminated according to the phenolic content in musts and massograms according to their sugar content. In fact, N-PLS (multi-way partial least squares) evidenced the good correlations existing between voltammetric data and Total Polyphenol Index measured by chemical methods. Similarly N-PLS showed a good relationship between mass outputs and sugar content. These results evidenced that multisensory systems

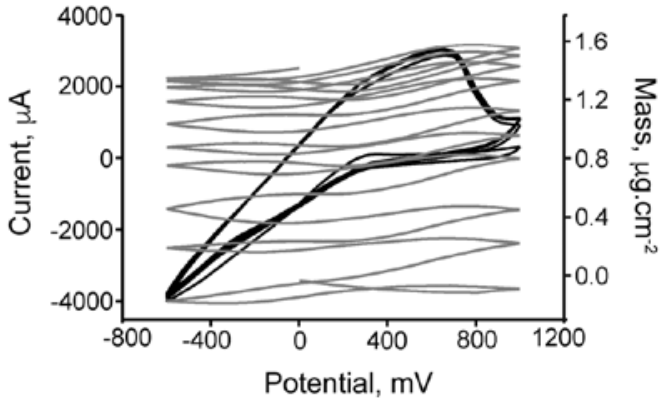

Fig. 3. Voltammetric (black line) and mass response (grey line) of the $\mathrm{CuPe}^{\mathrm{SO} 3} / \mathrm{PAH}$ sensor towards a must obtained from Cabernet grapes

formed by EQCM sensors are superior over arrays of mass or voltammetric sensors used separately [36].

\section{Arrays of biomimetic nanostructured biosensors} using phthalocyanines as electron mediators

As already stated in precedent paragraphs, the quality of the grapes is typically established on the basis of their phenolic and sugar content. The performance of a multisensor system dedicated to the analysis of grapes can be improved by combining non-specific sensors with biosensors containing enzymes selective to phenols or sugars. It has been claimed that arrays formed by biosensors can be advantageous because they combine the advantages of classical arrays of electrochemical sensors (that provide global information about the sample), with the specificity of the enzyme-substrate reaction typical of biosensors.

The immobilization of the enzyme onto the substrate is a key step in the development of efficient biosensors with high enzymatic activity. Layer by Layer (LbL) [41, 44] or Langmuir-Blodgett (LB) [45-51] techniques can be used to adsorb enzymes in lipidic membranes formed by fatty acids or phospholipids. This biomimetic environment contributes to the preservation of the enzymatic activity facilitating the electron transfer process. Furthermore, the biosensor sensitivity can be enhanced by the introduction in the LB or LbL film an appropriate electron mediator, which can facilitate the electron transfer between the redox enzyme and the electrode [52]. Phthalocyanines have demonstrated to be efficient electron mediators $[48,53]$.

A bioelectronic tongue based on nanostructured biosensors was developed and used to discriminate grapes according to their phenolic content. The multisensor system of three voltammetric sensing units was prepared using LB technique: one non-specific sensor based on a phthalocyanine and two biosensors formed by films of arachidic acid, a phthalocyanine (as the electron mediator) and the corresponding enzyme [54] 
Table 1. Results of the PLS-1 analysis

\begin{tabular}{|c|c|c|c|c|c|}
\hline Parameter & RMSEC $^{\mathrm{B}}$ & RMESP $^{\circ}$ & $\mathrm{R}^{2} \mathrm{C}^{0}$ & $\mathrm{R}_{\mathrm{P}}^{2}$ & LV \\
\hline $\mathrm{H}$ & 0.05800 & 0.06997 & 0.9822 & 0.9740 & 3 \\
\hline otal acidity & 0.04724 & 0.05948 & 0.9948 & 0.9918 & \\
\hline PI & 0.59340 & 0.67736 & 0.9731 & 0.9649 & \\
\hline
\end{tabular}

${ }^{3}$ Root mean square error of calibration; ' Root mean square error of prediction; ${ }^{\circ}$ Squared correlation coefficient in calibration; ${ }^{d}$ Squared correlation coefficient in prediction; 'Number of latent variables.

Tyrosinase and laccase were selected because they are phenoloxidases involved in the oxidation of phenols to the corresponding quinoid form [55].

When the array of sensors was immersed in grape juices, each sensor showed a characteristic electrochemical response towards musts obtained from five different varieties of grapes (Tempranillo, Garnacha, CabernetSauvignon, Prieto Picudo and Mencía). The voltammetric signals provided global information about the sample (as in classical multisensor systems) combined with specific information due to the specificity of the enzyme-substrate interaction. PCA of the obtained signals allowed a clear discrimination of the musts according to the variety of grape. Moreover, using PLS-1, excellent relationships were found between the responses provided by the biosensors and phenols and acids content provided by traditional chemical analysis carried out following international regulations [56]. As shown in Table 1, correlation coefficents $\left(\mathrm{R}^{2}\right)$ of $c a .0 .99$ were found in both calibration and validation, with residual errors close to zero and with an small number of latent variables.

\section{CONCLUSION}

Multisensor systems (Electronic Tongues) based on phthalocyanines are a good alternative to analyze grapes. Important differences between ETs are attributed to the type of sensor. CPE electrodes are simple to prepare and cheap, but their repeatability and sensitivity are low. In contrast, nanostructured LB or LbL sensors are highly sensitive but at a high cost.

The cross-selectivity of a multisensor system can be improved by constructing hybrid arrays combining nonspecific sensors with biosensors containing enzymes selective to specific compounds of interest, for instance phenoloxidases able to detect phenols. This can be of particular importance in the field of viticulture and enology where the content of certain compounds is directly related to the quality of wines and grapes.

\section{Acknowledgements}

Financial support from CICYT (Grant No. AGL201567482-R) and Junta de Castilla y León (VA-052A06) is gratefully acknowledged. CMP thanks the University of Valladolid for her PIF-UVa and CGH thanks for the grant of JCYL (BOCYL-D-24112015-9).

\section{REFERENCES}

1. De la Torre G, Claessens CG and Torres T. Chem. Commun. 2007; 20: 2000-2015.

2. Rodriguez-Morgade MS and Torres T. J. Porphyrins Phthalocyanines 2009; 13: 1-2.

3. The Porphyrin Handbook, Vols. 1-20, Kadish K, Smith KM and Guilard R. (Eds.) Academic Press: New York, 2003.

4. Jiang J. Functional Phthalocyanine Molecular Materials, Structure and Bonding (1st ed.), Vol. 135, Springer: Heidelberg, 2010.

5. Bill NL, Trukhina O, Sessler JL and Torres T. Chem. Commun. 2015; 51: 7781-7794.

6. Bouvet M, Gaudillat P and Suisse JM. J. Porphyrins Phthalocyanines 2013; 17: 913-919.

7. De la Torre G, Bottari G, Sekita M, Hausmann A, Guldi DM and Torres T. Chem. Soc. Rev. 2013; 42: 8049-8105.

8. Rodriguez-Mendez ML, Souto J, De Saja R, Martínez J and De Saja JA. Sens. Actuators, B. 1999 58: 544-551.

9. Rodriguez-Mendez ML, Gay M and De Saja JA. $J$. Porphyrins Phthalocyanines 2009; 13: 1159-1167.

10. Zagal JH, Griveau S, Santander-Nelli M, Granados SG and Bedioui F. J. Porphyrins Phthalocyanines 2012; 16: 713-740

11. Bouvet M, Gaudillat P and Suisse JM. J. Porphyrins Phthalocyanines 2013; 17: 628-635.

12. Rodriguez-Mendez ML, Medina-Plaza C, de Saja JA, Apetrei C and Muñoz R. In Sensor arrays based on phthalocyanines: New developments on nanostructured and biomimetic electrochemical sensors. Multisensor systems for chemical analysis: Materials and Sensors, Lvova L, Kirsanov D, Di Natale $\mathrm{C}$ and Legin A. (Eds.) Pan Stanford Publishing: Singapore, 2012; pp. 70-109.

13. Rodriguez-Mendez ML, Parra V, Apetrei C, Villanueva S, Gay M, Prieto N, Martínez J and De Saja JA. Microchim. Acta 2008; 163: 23-31.

14. De Saja JA and Rodriguez-Mendez ML. Adv. Colloid Interface Sci. 2005; 116: 1-11.

15. Alessio P, Pavinatto F, Oliveira O, De Saja JA, Constantino JC and Rodriguez-Mendez ML. Analyst. 2010; 135: 2591-2599.

16. Apetrei C, Nieto M, Rodríguez-Méndez ML and De Saja JA. J. Porphyrins Phthalocyanines 2011; 15: $1-10$.

17. Arrieta A, Rodriguez-Mendez ML and De Saja JA. Sens. Actuators, B. 2003; 95: 357-365.

18. Gay M, Rodriguez-Mendez ML and De Saja JA. Langmuir 2010; 26: 19217-19224. 
19. Apetrei C, Rodriguez-Mendez ML, Parra V, Gutierrez F and De Saja JA. Sens. Actuators, B. 2004; 103 $145-152$

20. Vlasov Y, Legin A, Rudnitskaya A, Di Natale C and D'Amico A. Pure Appl. Chem. 2005; 77: 1965-1983.

21. Sliwinska M, Wisniewska P, Dymerski T, Namiesnik J and Wardenci W. J. Agric. Food. Chem. 2014; 62 1423-1448.

22. Kirsanov D, Mednova O, Vietoris V, Kilmartin PA and Legin A. Talanta. 2012; 90: 109-116.

23. Smyth H and Cozzolino D. Chem. Rev. 2013; 113 1429-1440.

24. Electronic Noses and Tongues in the Food Industry, Rodriguez-Mendez ML. (Ed.) Academic Press: New York, 2016.

25. Heidarbeigi K, Mohtasebi SS, Serrano-Diaz J, Medina-Plaza C, Ghasemi-Varnamkhasti M, Alonso GI, Garcia-Rodriguez MV, Rafiee S, Rezaei K, Garcia-Hernandez C, De Saja JA and RodriguezMendez ML. Qual. Assur. Saf. Crop. 2015. DOI 10.3920/QAS2015.0591.

26. Apetrei C, Apetrei IM, Villanueva S, De Saja JA and Rodriguez-Mendez ML. Anal. Chim. Acta 2010; 663: 91-97.

27. Gutierrez N, Rodriguez-Mendez ML and De Saja JA. Sens. Actuators, B. 2001; 77: 437-442.

28. Ghasemi-Vamankhasti M, Rodriguez-Mendez ML, Mohtasebi SS, Apetrei C, Lozano J, Razavi SH, Ahmadi H and De Saja JA. Food Control. 2012; 25 : 216-224.

29. Mercante LA, Scagion VP, Pavinatto A, Sanfelice RC, Mattoso LHC and Correa DS. J. Nanomaterials. 2015; DOI: 10.1155/2015/890637.

30. Parra V, Hernando T, Rodriguez-Mendez ML and De Saja JA. Electrochim. Acta 2004; 49: 5177-5185

31. Ceto X, Apetrei C, Del Valle M and Rodriguez-Mendez ML. Electrochim. Acta 2014; 120: 180-186.

32. Gay M, Apetrei C, Nevares I, Del Alamo M, Zurro J, De Saja JA and Rodriguez-Mendez ML. Electrochim. Acta 2010; 55: 6782-6788.

33. Boulton R. Am. J. Enol. Vitic. 2001; 52: 67-87.

34. Glories Y. Progrès Agricole et Viticole 2001; 118 347-350.

35. Moreno-Codinachs L, Kloock JP, Schöning MJ, Baldi A, Ipatov A, Bratov A and Jiménez-Jorquera C. Analyst. 2008; 133: 1440-1448.

36. García-Hernández C, Medina-Plaza C, GarcíaCabezón C, Martín-Pedrosa F, Del Valle I, De Saja JA and Rodríguez-Méndez ML. Sensors 2015; 15: 29233-29249.

37. Parra V, Arrieta A, Fernández-Escudero JA, García H, Apetrei C, Rodríguez-Méndez ML and De Saja JA. Sens. Actuators, B. 2006; 115: $54-61$.
38. Rodriguez-Méndez ML, Medina-Plaza C, GarcíaHernández C, De Saja JA, Fernández-Escudero JA, Barajas-Tola E and Medrano G. IEEE Sensors Journal 2015; Sensors, 2014 IEEE: 2139-2142.

39. Medina C, Revilla G, Muñoz R, Fernández-Escudero JA, Barajas E, Medrano G, De Saja JA and Rodriguez-Méndez ML. J. Porphyrins Phthalocyanines $2014 ; 18$ : 76-86.

40. Ferreira M,Zucolotto V, Oliveira ON Jr and Wohnrath K. Encyclopedia of Nanoscience and Nanotechnology 4, Nalwa HS. (Ed.) American Scientific Publishers: Los Angeles, 2003; pp. 441-460.

41. Fernandes EGR, Brazaca LC, Rodríguez-Méndez ML, De Saja JA and Zucolotto V. Biosens. Bioelec tron. 2011; 26: 4715-4719.

42. Alessio P, Apetrei C, Rubira RJG, Constantino CJL, Medina-Plaza C, De Saja JA and RodríguezMéndez ML. I Nanosci. Nanotechnol. 2014; 14 6754-6763.

43. Ozturk ZZ, Kilinc N, Atilla D, Gurek AG and Ahsen V. J. Porphyrins Phthalocyanines 2009; 13: 1179-1187.

44. Dos Santos V, Dos Santos M, De Jesus CG, Fujiwara ST, Garcia JR, Pessoa CA and Wohrath K. Int. J. Electrochem. Sci. 2013; 8: 10601-10620.

45. Cajab J, Soloducho J and Nowakowska-Oleksy A. Sens. Actuators, B. 2010; 143: 508-515.

46. Zanon NCM, Oliveira ON Jr and Caseli L. J. Colloid Interface Sci. 2012; 373: 69-74.

47. Wang KH, Syu MJ, Chang CH and Lee YL. Sens. Actuators, B. 2012; 164: 29-36.

48. Pavinatto FJ, Femandes EGR, Alessio P, Constantino CJL, De Saja JA, Zucolotto V, Apetrei C, Oliveira ON Jr and Rodriguez-Mendez ML. J. Mater: Chem. 2011; 21: 4995-5003.

49. Goto TE, Lopez RF, Oliveira ON Jr and Caseli L. Langmuir 2010; 26: 11135-11139.

50. Hodak J, Etchenique R, Calvo EJ, Singhal K and Bartlett PN. Langmuir 1997; 13: 2708-2711.

51. Ohnuki H, Saiki T, Kusakari A, Endo H, Ichihara M and Izumi M. Langmuir 2007; 23: 4675-4681.

52. Harper A and Anderson MR. Sensors. 2010; 10: 8248-8274.

53. De Alburquerque YDT and Ferreira LF. Anal. Chim. Acta 2007; 596: 210-221.

54. Medina-Plaza C, De Saja JA and Rodriguez-Mendez ML. Biosens. Bioelectron. 2014; 57: 276-283.

55. Sotomayor MDT, Tanaka AA and Kubota LT. Anal. Chim. Acta 2002; 455: 215-223.

56. Compendium of International Methods of Analysis of Wines and Musts, Vol. 2. OIV: Paris, 2013. 


\subsection{Paper II}

Monitoring the phenolic ripening of red grapes using a multisensor system based on metal-oxide nanoparticles.

By Celia García-Hernández, Cristina Medina-Plaza, Cristina GarcíaCabezón, Yolanda Blanco, José A. Fernández-Escudero, Enrique BarajasTola, Miguel A. Rodríguez-Pérez, Fernando Martín-Pedrosa and Maria Luz Rodríguez-Méndez.

Frontiers in Chemistry 6 (2018) 131.

(doi: 10.3389/fchem.2018.00131) 


\subsubsection{Motivation and objective}

The performance of electrochemical sensors and biosensors has been greatly improved by using nanotechnology. This field has received increasing attention from researchers in analytical chemistry applications. There are many nanomaterials which have been implemented in many applications, such as sensing or drug delivery, among others. On this matter, nanoparticles are excellent candidates, due to their small size, as they provide unique physical, chemical and electronic properties not found in bulk materials. For instance, metal nanoparticles exhibit excellent conductivity and electrocatalytic properties related to the formation of mixed valence states on their surface that help to enhance the surface area, facilitate the electron transfer between redox species and electrode surface, improve the sensitivity and selectivity of sensors, as well as the signal-to-noise ratio; metal oxide nanoparticles can be used to immobilize biomolecules due to their biocompatibility; while semiconductor nanoparticles have been applied as sensor labels for electrochemical detection.

Therefore, when using nanoparticles in electrochemical sensors, it is understandable to expect a decrease in the redox potentials of electrochemical reactions, improved reversibility or increased responses. Moreover, for biosensing applications with enzymes, nanoparticles can ameliorate the lack of electrical contact between the enzyme and the electrode, which is practically non-existent as enzyme active sites are impeded by insulating protein shells. The conductivity of metal, metal oxide and semiconductor nanoparticles enhances the electron transfer between the active sites and the surface electrodes acting as mediators or electrical wires.

In short, metal nanoparticles, metal oxide nanoparticles and semiconductor nanoparticles have mainly been used in electrochemical sensor systems for the following purposes: catalysis of electrochemical reactions, immobilization of biomolecules, improvement of electron transfer, labelling biomolecules and acting as reactant.

They also have the advantage that they can be deposited onto electrode surfaces by many methods, including covalent bond, electrodeposition or physical adsorption, among others.

As previously stated, the optimal date of harvesting is a point that concerns wine growers. The main parameter to monitor the maturity of grapes is the sugar content ( ${ }^{\circ} \mathrm{Brix}$ ), although other parameters, such as the phenolic content (TPI) and total acidity (TA), are also 
relevant as they can strongly influence the final organoleptic characteristics of wines. For example, many oenologists use the relation ${ }^{\circ}$ Brix/TA to decide the optimal maturity day, although there is no biochemical relationship between the acidity loss and the sugar increase.

Metal oxide nanoparticles have been widely used in the detection of many analytes, such as $\mathrm{H}_{2} \mathrm{O}_{2}$, glucose, dopamine, bis-phenol A, catechol, ascorbic acid, fructose, cysteine, etc., but they have never been applied to electronic tongues, which is the novelty of this work, together with the purpose of implementing a multisensor system to study grape ripeness.

Therefore, in this work, we have developed an electronic tongue based on metal oxide nanoparticles $\left(\mathrm{CeO}_{2}, \mathrm{NiO}\right.$ and $\left.\mathrm{TiO}_{2}\right)$ to monitor grape ripeness from véraison to over-ripening in terms of phenolic maturity. Statistical methods were used to discriminate between grape samples by means of PCA and, finally, correlations were established between the chemical parameters of the analysed grapes and the data obtained with the electronic tongue using PLS-1.

\subsubsection{Summary of the research work}

Sensors were prepared by using a simple, previously reported method based on carbon paste electrodes (CPE), where phthalocyanines were mixed with graphite powder and a mineral oil (nujol). In this case, phthalocyanines were replaced by metal oxide nanoparticles. Therefore, the array of sensors that formed the electronic tongue was composed of nickel (II) (NiONP-CPE), titanium (IV) ( $\mathrm{TiO}_{2} \mathrm{NP}-\mathrm{CPE}$ ) and cerium (IV) ( $\left.\mathrm{CeO}_{2} \mathrm{NP}-\mathrm{CPE}\right)$ oxide nanoparticles and one nonmodified carbon paste electrode (C-CPE). Plastic syringes were used as support for the carbon paste. TEM images showed that MONPs were spherical, with a homogeneus distribution and an average size ranging from 20 to $50 \mathrm{~nm}$.

Grape samples were provided by the vineyards Bodega Cooperativa de Cigales and the Instituto Tecnológico Agrario de Castilla y León (ITACyL). Eight different varieties were analyzed: Tempranillo, Garnacha, Cabernet, Prieto Picudo, Juan García, Mencía Secano, Mencía Regadío and Rufete. All samples were collected weekly from véraison to maturity, and several grapes bunches were also left in the vineyards and were collected two extra weeks after maturity (overripeness). The varieties Tempranillo, Garnacha and Cabernet were considered mature 6 weeks after véraison and the rest of the varieties after 5 weeks. The Oenological Centre of Castilla y 
León analyzed the chemical parameters of the grapes and the Brix degree, TPI and total acidity were provided. These parameters were analyzed weekly as was the ${ }^{\circ}$ Brix/TA, since this relationship is used by several wine growers to establish the optimal harvesting date. The results showed that TA decreased during the ripening process, sugar content increased and TPI varied irregularly, but increased drastically just after véraison. For Garnacha, Cabernet and Tempranillo grapes, the ${ }^{\circ}$ Brix/TA ratio increased weekly and reached stabilization after week 5 , indicating the proximity of grape maturity and grapes were then harvested in week 6. Mencía Secano, Mencía Regadío, Prieto Picudo, Juan García and Rufete ripened faster and were harvested in week 5.

The changes due to over-ripening demonstrated that TA values were almost constant, but the ${ }^{\circ}$ Brix continued to increase, so the optimal ripening date was not reached until week 7 or 8 (depending on the variety). As the TPI trend was so irregular, this result showed that, using traditional methods, we are not able to assess phenolic maturity.

In order to find out if the developed electronic tongue based on metal oxide nanoparticles was able to monitor the phenolic ripening, the e-tongue was tested in catechol, vanillic acid, caffeic acid and pyrogallol, which are all phenols present in grapes. The voltammetric responses showed that metal oxide nanoparticles induced an increase in the intensities and/or a decrease in redox potentials, making the process more easily reversible. Each sensor showed a characteristic response that provided a degree of cross-selectivity in the responses, which is advantageous for its application as a multisensor system. The electrocatalytic effect was more strongly pronounced in the mono-phenol (vanillic acid) and di-phenols (catechol and caffeic acid) than in the tri-phenol (pyrogallol). The detection limits were found to be $10^{-8} \mathrm{M}$, which is much lower than those reported for traditional carbon paste electrodes, but are similar or comparable to detection limits obtained with more sophisticated sensors constructed with nanostructured Langmuir-Blodgett or LbL films. Additionally, PCA analysis evidenced that the electronic tongue was able to discriminate phenols according to the number of hydroxyl groups present in the molecule (mono, di or tri).

The electronic tongue was then immersed in grapes collected weekly from véraison during 7 or 8 weeks from véraison (8 weeks for Cabernet, Garnacha and Tempranillo and 7 weeks for the rest). Voltammograms showed oxidation peaks related to redox species present in the musts (mainly phenols). The peaks observed at negative potentials were due to the acidity of the samples. The different electrocatalytic properties of the metal oxide nanoparticles used was 
reflected in the responses. For example, NiONP-CPEs were more sensitive to phenol redox processes, whereas $\mathrm{TiO}_{2} \mathrm{NP}-\mathrm{CPES}$ were sensitive to the acidity.

PCA analysis was carried out for each variety of grape and the results were very interesting. For the varieties Mencía Secano, Mencía Regadío, Prieto Picudo, Juan Garcia and Rufete, the clusters corresponding to the first 4 weeks were located drawing a kind of circle that was "closed" passing through the sample of week 5 (ripening week), located relatively near the week 1 sample, and then the samples of weeks 6 and 7 were located near weeks 2 and 3 , respectively, following a kind of spiral tendency. This result was also observed for the Cabernet, Garnacha and Tempranillo varieties, where the week 6 sample (ripening week) was repeating the same behavior as the week 5 sample described above, with the over-ripening samples also following a kind of spiral tendency. These results indicated that the optimal phenolic maturity point is connected to when the circle is closed and before samples start to follow a spiral tendency.

Finally, regression models were performed by PLS-1 to find linear correlations between the chemical parameters ( ${ }^{\circ} \mathrm{Brix}, \mathrm{TA}$ and TPI) and the data obtained with the electronic tongue. For each grape variety, good correlations were found between the voltammetric signals and the phenolic content (TPI), with a low number of latent variables (2-4), correlation coefficients of about 0.8 and low errors. ${ }^{\circ}$ Brix and TA, which are the parameters usually used to monitor the maturity of grapes, gave regression models with lower correlations, showing a higher number of latent variables, but with low errors and similar coefficient of correlations. 



\section{OPEN ACCESS}

Edited by:

Larisa Lvova,

Università degli Studi di Roma Tor

Vergata, Italy

Reviewed by:

Nicols I Jaffrezic-Renault, Claude Bernard University Lyon 1 ,

France

Prathik Roy

University of Canterbury, New Zealand Cocilia Jimenez-Jorquera,

Consejo Superior de investigaciones Cientficas (CSIC), Spain

*Correspondence: Maria L Rodriguez-Mandez muzasili.ua es

Specialty section: This article was submitted to Analytical Chamistry, a section of the jouma Frontiers in Chemistry

Received: 23 January 2018 Accepted: 09 Aprit 2018 Published: 24 April 2018

Citation:

Garcla-Hernandez C, Madina-Plaza C, Garcia-Cabezon C, Blanco Y, Farnandez-Escudero JA Baraias-Tola E, Rodriguez-Porez MA Martin-Pedrosa Fand Rodriguez-Mendez ML (2018) Monitoring the Phenolic Pipening of Red Grapes Using a Multisensor

System Based on Motal-Oxide Nanoparticles. Front Chem 6:131. dol: $10.3389 /$ fchem.2018.00131

\section{Monitoring the Phenolic Ripening of Red Grapes Using a Multisensor System Based on Metal-Oxide Nanoparticles}

Celia Garcia-Hernandez', Cristina Medina-Plaza ', Cristina Garcia-Cabezon ${ }^{2}$, Yolanda Blanco ${ }^{2}$, Jose A. Fernandez-Escudero ${ }^{3}$, Enrique Barajas-Tola ${ }^{4}$,

Miguel A. Rodriguez-Perez ${ }^{1}$, Fernando Martin-Pedrosa ${ }^{2}$ and Maria L. Rodriguez-Mendez ${ }^{\text {* }}$

'Group UVaSens, Department of Inorganic Chemistry, Escuela de Ingenierias Industriales, Universidad de Valladolid, Valladolid, Spain, ${ }^{2}$ Group UVasens, Department of Materials Sciance, Universidad de Valladollid, Valladolid, Spain, ${ }^{3}$ Estacion Enologica de Castilla y Leon, Valladolld, Spain, "ITACYL, Valladolid, Spain

The maturity of grapes is usually monitored by means of the sugar concentration. However, the assessment of other parameters such as the phenolic content is also important because the phenolic maturity has an important impact on the organoleptic characteristics of wines. In this work, voltammetric sensors able to detect phenols in red grapes have been developed. They are based on metal oxide nanoparticles $\left(\mathrm{CeO}_{2}, \mathrm{NiO}\right.$, and $\left.\mathrm{TiO}_{2},\right)$ whose excellent electrocatalytic properties toward phenols allows obtaining sensors with detection limits in the range of $10^{-8} \mathrm{M}$ and coefficients of variation lower than $7 \%$. An electronic tongue constructed using a combination of the nanoparticle-based sensors is capable to monitor the phenolic maturity of red grapes from véraison to maturity. Principal Component Analysis (PCA) can be successfully used to discriminate samples according to the ripeness. Regression models performed using Partial Least Squares (PLS-1) have established good correlations between voltammetric data obtained with the electrochemical sensors and the Total Polyphenolic Index, the Brix degree and the Total Acidity, with correlation coefficients close to 1 and low number of latent variables. An advantage of this system is that the electronic tongue can be used for the simultaneous assessment of these three parameters which are the main factors used to monitor the maturity of grapes. Thus the electronic tongue based on metal oxide nanoparticles can be a valuable tool to monitor ripeness. These results demonstrate the exciting possible applications of metal oxide nanoparticles in the field of electronic tongues.

Keywords: electronic tongue, grape, ripening, phenolic maturity, metal oxide nanoparticles

\section{INTRODUCTION}

Grapes must be harvested at the optimal maturity point. The sugar content has a direct influence on the alcoholic degree of wines and it is the analytical indicator commonly used to decide the harvest date (OIV, 2013). It is periodically assessed using fast and simple density measurements and it is expressed as degree Brix ( $\left.{ }^{\circ} \mathrm{Brix}\right)$. The variation of the acidity along ripening is also an important parameter which is expressed as Total Acidity (TA) (Boss et al., 2014). 
The phenolic content of grapes also changes during ripening and the phenolic maturity has a direct impact on the organoleptic characteristics of wines, and it would be desirable to assess it routinely. However, the main changes in the phenolic content take place in skins and seeds, and the assessment of phenolic maturity requires the previous separation of seeds and skins using long and complex methods (Xu et al., 2011; Meléndez et al., 2013; Nogales-Bueno et al., 2014; Sharma et al., 2015). Oenologists require new methods capable to measure the phenolic maturity of grapes with simple and direct methods.

Besides, it would be of great interest to develop new technologies able to detect simultaneously the sugar content, acidity and phenolic composition. Such a method would help to take faster decisions about the optimal harvest date. One possible approach can be the use of holistic methods, where complex signals obtained from an instrument such as GC-MS, FTIR or NMR, are processed using multivariate methods. These holistic methods have been successfully used to classify wines according to quality, to monitor fermentation, to detect wine spoilage, etc. (Buratti et al., 2011; Cozzolino et al., 2012; Godelmann et al., 2014).

Electronic tongues (ET) are a new class of instruments which are gaining interest in the food industry (Cosio et al., 2012; Kirsanov et al., 2012; Lvova et al., 2014; Rodríguez-Méndez, 2016). They are multisensor systems where an array of sensors is combined with a multivariate data software. The most common sensors used in ETs dedicated to the analysis of wines are electrochemical (amperometric or voltammetric). They have been widely used to discriminate wines according to the variety of grape, the vintage, the type of barrel used for aging etc. (Gutiérrez et al., 2010; Prieto et al., 2011; Apetrei et al., 2012; Cetó et al., 2017; Rudnitskaya et al., 2017). In spite of the interest of winemakers to have available objective methods to determine the harvesting date and the quality of grapes, ETs have been rarely applied to the quality control of grapes or their musts (Codinachs et al., 2008; Gutiérrez et al., 2011; Campos et al., 2013; Medina-Plaza et al., $2014 a, b)$.

Nowadays, it is clear that the performance of ETs can be improved by introducing in the array sensing units specifically dedicated to a particular application. Electrodes with enhanced performances can be obtained using new sensing materials linked to nanotechnology. Metal nanoparticles have been used in the fabrication of electrochemical sensors due to their electrocatalytic properties which are related to the formation of mixed valence states on their surface (Campbell and Compton, 2010; Saha et al., 2012; Lin et al., 2013). Metal nanoparticles have demonstrated their capability to catalyze the oxidation/reduction of organic acids and phenols commonly present in wines. The oxidation of phenols occurs at lower potentials, whereas the presence of metallic nanoparticles increases the reactivity of acids and enhances the intensity of the anodic wave at negative potentials (Medina-Plaza et al., $2015 \mathrm{a}, \mathrm{b})$. In spite of this interest, metal nanoparticles have rarely been included as sensing materials in electronic tongues (Gutiérrez et al., 2013; Sharpe et al., 2014; Mercante et al., 2015). Up to now, the sensing capabilities of metal oxide nanoparticles (MONPs) have never been tested in electronic tongues.
The aim of this work was to develop an electronic tongue (ET) formed by sensors based on metal oxide nanoparticles $\left(\mathrm{CeO}_{2}, \mathrm{NiO}\right.$, and $\left.\mathrm{TiO}_{2},\right)$ and to evaluate their capabilities to detect phenols. Using chemometric techniques such as Principal Component Analysis (PCA), the ET was used to monitor the ripeness of 8 different varieties of grapes, from véraison (the onset of ripening) to post-maturity. Mathematical models were built to establish correlations between the phenolic index measured by traditional chemical techniques and the results of the ET. PLS-1 was used to predict the polyphenol index of the grapes along ripening. The possibility to obtain information about other classical indicators of maturity such as the ${ }^{\circ} \mathrm{Brix}$ and Total Acidity was also analyzed.

\section{MATERIALS AND METHODS}

Metal oxide nanoparticles of Titanium (IV), Cerium (IV), and Nickel (II) $\left(\mathrm{TiO}_{2} \mathrm{NP}, \mathrm{CeO}_{2} \mathrm{NP}, \mathrm{NiONP}\right)$ were purchased from Sigma-Aldrich. All reagents and solvents were of high purity (Sigma-Aldrich). Deionized water $\left(18.2 \mathrm{M} \Omega \cdot \mathrm{cm}^{-1}\right)$ was obtained from a Millipore MilliQ purifier.

Electron Microscope TEM images were recorded using a high-resolution electron microscope (HRTEM: JEOL JEM 2200) (Tokyo, Japan) operating at an accelerating voltage of $200 \mathrm{kV}$. Sample images were processed using Image J image processing software (public software)

Eight varieties of red grapes typical from the CastillaLeon region (Spain) were included in the study: Tempranillo, Garnacha, Cabernet-Sauvignon, Prieto Picudo, Mencia Regadio, Mencia Secano, Rufete, and Juan Garcia. Grapes were grown and harvested by the Agrotechnological Institute of Agriculture of the Regional Government (ITACyL) and the cellar Bodega Cooperativa de Cigales. Berries were collected in a weekly basis from véraison until grapes were completely mature. Ripeness rate was different from one variety to another. For this reason, the official harvest date indicated by traditional chemical parameters, varied from one variety of grape to another. Grapes from Tempranillo, Garnacha, and Cabernet Sauvignon were considered matured 6 weeks after véraison. The rest of varieties attained the optimal ripeness 5 weeks after véraison. In all cases several grape bunches were left in the vine and two extra samplings were collected in successive weeks in order to analyze over-ripened grapes.

Musts were prepared from 100 berries representative of the ripening state of the vineyard. Once collected, grapes were crushed, and peels and seeds were separated by decantation. $50 \mathrm{~mL}$ aliquots of musts thus obtained, were frozen at $-20^{\circ} \mathrm{C}$ until used. Total Polyphenol Index (TPI), Brix degree $\left({ }^{\circ} \mathrm{Brix}\right)$ and Total Acidity (TA) were analyzed following international standard methods (OIV, 2013; Boss et al., 2014). TPI was determined by diluting the samples with distilled water in a 1:100 ratio and measuring the absorbance directly at $280 \mathrm{~nm}$ (using a quartz cuvette of $10 \mathrm{~mm}$ optical path). The value of the I $280 \mathrm{~nm}$ (TPI) was calculated by multiplying the absorbance $\times 100$. The total acidity is the sum of its titratable acidities when it is titrated to $\mathrm{pH} 7$ against a standard alkaline solution. Carbon dioxide is not included in the total acidity. The total acidity is usually expressed in grams of tartaric acid per liter. 
Brix measurements are taken with an Atago WM-7 digital refractometer.

A multisensor system consisting of 4 Carbon Paste Electrodes (CPE) was formed. Three electrodes were modified with metal oxide nanoparticles including $\mathrm{NiO}$ (NiONP-CPE), $\mathrm{TiO}_{2}$ $\left(\mathrm{TiO}_{2} \mathrm{NP}-\mathrm{CPE}\right)$, and $\mathrm{CeO}_{2}\left(\mathrm{CeO}_{2} \mathrm{NP}-\mathrm{CPE}\right)$. One unmodified carbon paste electrode (C-CPE) was also included in the array. Electrodes were prepared following the classical method by mixing graphite with the corresponding metal oxide nanoparticle (15\% w/w of the MONP) Nujol was used as the binder until a paste was obtained. The paste was packed in a $1 \mathrm{~mL}$ plastic syringe and compressed. A metallic copper wire was used as a contact (Apetrei et al., 2011). The size of MNOPS ranged from 20 to $50 \mathrm{nM}$. Figure 1 illustrates the shape and size of $\mathrm{TiO}_{2} \mathrm{MNPs}$ As observed in the TEM image, MONPs were spheric with an homogeneous distribution of sizes that coincided with the size labeled by the supplier.

Electrochemical experiments were carried out using a potentiostat (Autolab. Metrohm, USA) combined with a multiplexor system. CPEs (surface of $2 \mathrm{~mm}$ in diameter) served as working electrodes, with an $\mathrm{Ag} \mid \mathrm{AgCl} / \mathrm{KCl} 3 \mathrm{~mol} \cdot \mathrm{L}^{-1}$ electrode acting as a reference electrode and a platinum wire as the counter

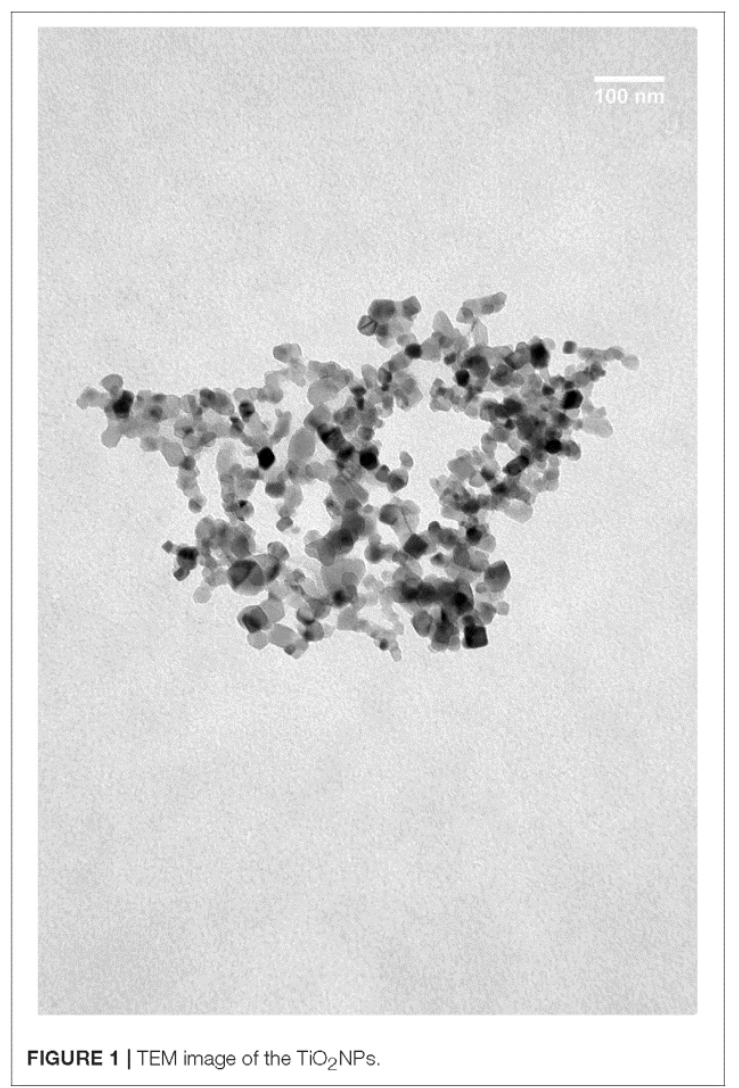

electrode. The MONPs sensors were able to provide responses toward aqueous solutions of phenolic acids (vanillic and caffeic acids) and polyphenols (catechol and pyrogallol) present in wines. The electrocatalytic properties and the detection limits (LD) were evaluated. The calibration curves were constructed measuring standard solutions with concentrations ranging from $10^{-4}$ to $10^{-6} \mathrm{~mol} \cdot \mathrm{L}^{-1}$. LDs were calculated following the $3 \mathrm{SD} / \mathrm{m}$ criterion. The reproducibility of data provided by the MONPs sensors was evaluated by comparing data provided by three sensors measuring standard solutions in different days. Cyclic voltammograms were registered at a sweep rate of $0.1 \mathrm{~V} \cdot \mathrm{s}^{-1}$ from -0.8 to $+1.0 \mathrm{~V}$. Four replicas of each sample were measured. Principal Component Analysis (PCA) and Partial Least Squares (PLS) models were built using the software Matlab v5.3. (The Mathworks Inc., Natick, MA, USA).

Prior to perform the statistical analysis, the number of variables was reduced using a data reduction technique based on predefined response "bell-shaped-windowing" curves called "kernels" (Medina-Plaza et al., 2015b). In this method, each voltammogrammetric curve is multiplied by 10 smooth, bellshaped windowing functions defined as:

$$
K_{i}\left(V_{j}\right)=\frac{1}{1+\left(\frac{V_{j}-c_{i}}{a_{i}}\right)^{2 b_{i}}}
$$

where $a_{i}, b_{i}$, and $c_{i}$ define the width, shape, and center of the different windowing functions $K_{i}$. Subsequently, data were integrated with respect to voltage. As a result, each voltammogram was reduced to a vector of 10 variables that were used as the input data source for statistical analysis.

\section{RESULTS AND DISCUSSION}

\section{Monitoring Ripeness Using Chemical} Parameters

Ripening was monitored by measuring ${ }^{\circ}$ Brix, TA, and TPI in a weekly basis (from véraison). These parameters were used by oenologists to establish the optimal date of harvest. The ${ }^{\circ} \mathrm{Brix} / \mathrm{TA}$ ratio was also calculated. This parameter is also commonly used to decide the optimal maturity date even if there is no biochemical relationship between the acidity loss and the sugar increase. The complete list of parameters is collected in Supplementary Table. Details can be observed in Figure 2 for variety Tempranillo.

As observed in the Figure 2, TA and ${ }^{\circ}$ Brix followed the expected trend: TA decreased along the ripening process, while sugar content increased. ${ }^{\circ}$ Brix/TA ratio increased accordingly. These values were stabilized after week 5, indicating the proximity of the end of ripeness, and grapes were considered mature and harvested in week 6. Similar trends were observed in Garnacha and Cabernet-Sauvignon that also reached the maturity in week 6. Mencia Secano, Mencia Regadio, Rufete, and Juan Garcia gapes, matured at a faster rate, and were considered ripened and harvested in week 5 .

In order to study the changes in over-ripened grapes, some bunches were kept in the plant during 2 more weeks. During 
Paper II: Monitoring the phenolic ripening of red grapes using a multisensor system based on metal-oxide nanoparticles.

these 2 extra weeks, TA values were almost constant, whereas ${ }^{\circ}$ Brix raised drastically in Cabernet and Garnacha grapes, indicating that optimal ripening was not attained until weeks 7 or 8 .

TPI values increased sharply just after the veraison. The following weeks, TPI varied irregularly. These results evidenced the difficulty to assess the phenolic maturity using traditional

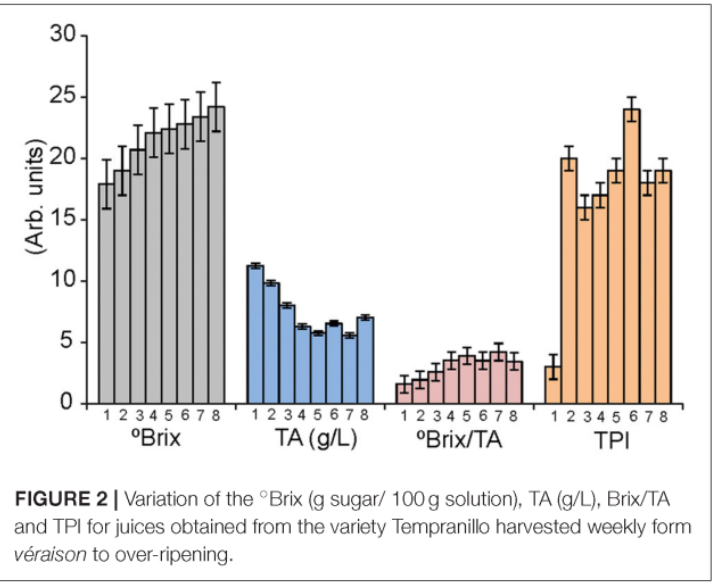

TPI measurements in pulps. It is therefore important for wine producers to develop new methods to define the peak of ripeness and in particular of the phenolic ripeness (which nowadays is difficult to assess)

\section{Electrochemical Characterization of the NP-Based Electrode. Electrocatalytic Activity Toward Phenols}

The objective of this work was to develop a multisensory system based on metal oxide nanoparticles to monitor the phenolic ripening. As a first task, the response of the MONP-CPE electrodes toward four phenols present in musts (vanillic acid, catechol, caffeic acid, and pyrogallol $10^{-4} \mathrm{M}$ ), was evaluated (Figure 3). The response of the C-CPE electrode is also shown for comparison purposes.

The responses on MONP-CPEs were consistent with those already reported in bare CPE electrodes (Kilmartin et al., 2001), but the presence of MONPs caused shifts in the position of the peaks to lower potentials and/or increases in the intensity of the peaks. This effect could be attributed to the excellent electrocatalytic activity of MONPs due to the mixed valence state found at the nanoparticle surface (Medina-Plaza et al., 2015b). Depending on the nature of the MONPs used as modifiers, the responses toward a certain compound was more or less enhanced. For instance, the intensity of the redox peaks produced by the oxidation/reduction observed in vanillic
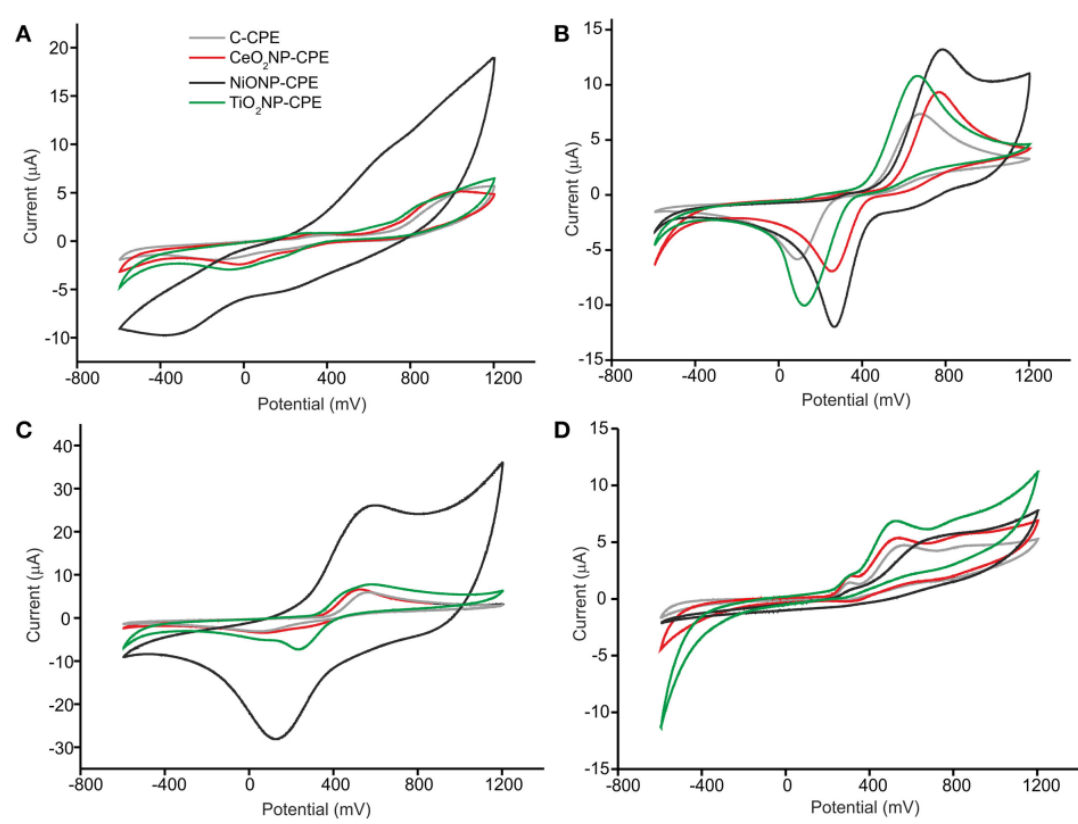

FIGURE 3 | CV of (A) vanillic acid, (B) catechol, (C) caffeic acid, and (D) pyrogaloll at C-CPE, $\mathrm{CeO}_{2} \mathrm{NP}-\mathrm{CPE}$, NiONP-CPE, and TiO 2 NP-CPE electrodes Concentration of $10^{-4} \mathrm{M}$. Scan rate $100 \mathrm{mV} \cdot \mathrm{s}^{-1}$ 

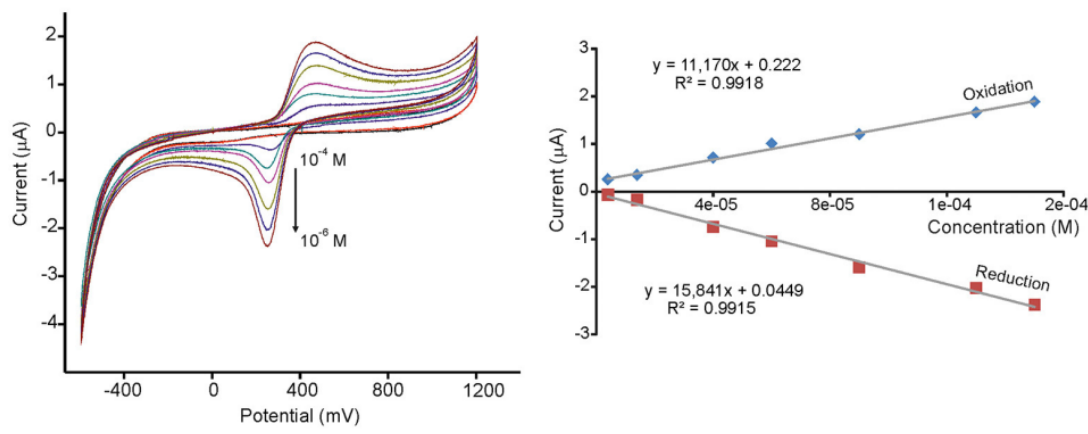

FIGURE 4 | CVs and calibration curves of $\mathrm{TiO}_{2} \mathrm{NP}-\mathrm{CPE}$ immersed in increasing concentrations of caffeic acid (from $4 \cdot 0 \cdot 10^{-6} \mathrm{M}$ to $1 \cdot 5 \cdot 10^{-4} \mathrm{M}$ ). Scan rate $0.1 \mathrm{~V} \cdot \mathrm{s}-1$.

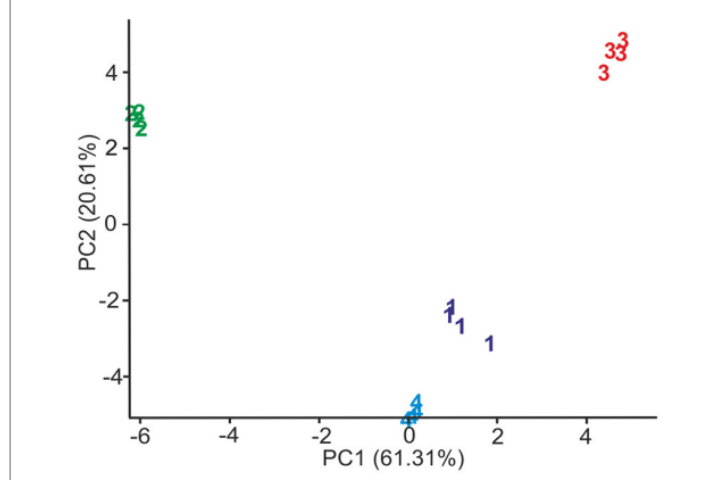

FIGURE 5 | Scores plot of the first two principal components of the PCA model calculated from voltammograms registered when electrodes were immersed in 1: Catechol, 2: Pyrogallol, 3: Vanillic acid and 4: Caffeic acid

acid and catechol at the graphite electrode surface increased drastically in the presence of NiONPs. The electrocatalytic effect was not so remarkable at $\mathrm{TiO}_{2} \mathrm{NP}-\mathrm{CPE}$ or $\mathrm{CeO}_{2} \mathrm{NP}$ CPEs electrodes. It is also worth noting that the electrocatalytic effect was more intense in the mono-phenol (vanillic acid) or di-phenols (catechol and caffeic acid) than in the tri-phenol (pyrogallol)

Limits of detection (LD) were calculated from the slope of the curves of the redox peaks registered at concentrations ranging from $10^{-4}$ to $10^{-6} \mathrm{M}$, using $3 \mathrm{SD} / \mathrm{m}$ method, where $S D$ is the standard deviation of the current density. LDs as low as $10^{-8} \mathrm{M}$ could be attained, which are much lower than those typically found in carbon electrodes (Kilmartin et al., 2001). The results are illustrated in Figure 4 for $\mathrm{TiO}_{2} \mathrm{NP}-\mathrm{CPE}$ immersed in caffeic acid. Reproducibility was also evaluated by measuring repeatedly the standard solutions and Variation coefficients (CV) lower than $7 \%$ were found in all cases. From the above results it can be concluded that modification with metal oxide nanoparticles drastically increases the sensibility of voltammetric electrodes toward phenols present in musts.

As stated before, each sensor showed a characteristic response which is linked to the electrocatalytic properties of the type of nanoparticle introduced in the carbon paste. This crossselectivity can be used to construct a multisensor system coupled to a pattern recognition software able to provide a pattern or "digital fingerprint" for each sample studied.

Figure 5 shows the Principal Component Analysis (PCA) scores plot obtained from the responses of the array to $10^{-4}$ $\mathrm{M}$ solutions of phenols. The PCA showed four distinct clusters in the plot of the two first principal components (PC1 61.31\% and PC2 20.61\%). The position of the clusters was related to the number of hydroxyl groups in the molecule. The monophenol (vanillic acid) was located at positive PC1 values, diphenols (catechol and caffeic acid) in the central part of the diagram and the triphenol (pyrogallol) on the left side corresponding to positive PC2 values.

\section{Monitoring the Ripening of Grapes With an Array of MONP-Based Electrodes}

The capacity of the electronic tongue to monitor the phenolic maturity of grapes was evaluated by analyzing juices obtained from grapes collected from véraison, in a weekly basis, during 7-8 weeks. Due to the complexity of the samples and in order to improve the repeatability, musts were diluted 1:2 with deionized water. Coefficients of variation lower than $7 \%$ were obtained in all cases.

Figure 6 illustrates the responses obtained using the NiONP$\mathrm{CPE}$ and $\mathrm{TiO}_{2} \mathrm{NP}-\mathrm{CPE}$ sensors immersed in musts obtained from grapes of the variety Garnacha collected during 7 weeks after véraison.

As illustrated in the figure, voltammograms registered in must showed peaks in the $0.6-0.8 \mathrm{~V}$ region, produced by components with redox activity (mainly phenols). Other components present in musts also influenced the overall response. For instance, the peaks observed at negative potentials were affected by the acidity of the samples. The array of sensors showed an interesting crossselectivity that was due to the different catalytic properties of 


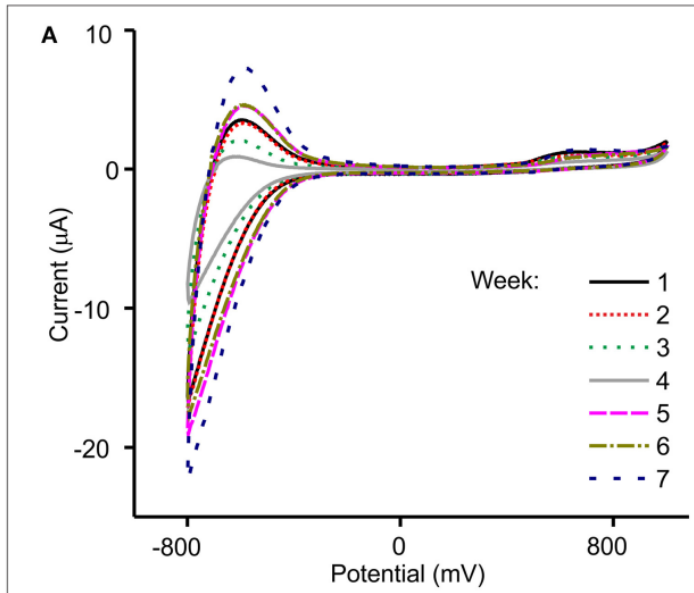

B

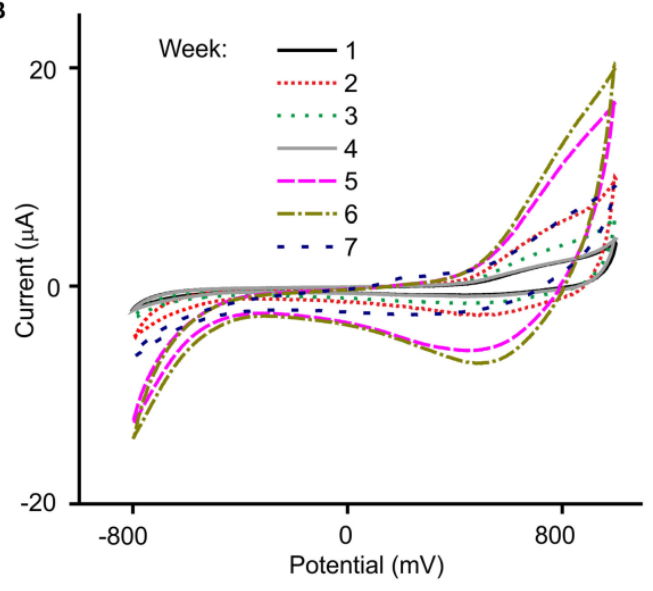

FIGURE 6 | Voltammetric responses of (A) $\mathrm{TiO}_{2}$ NP-CPE and (B) NiONP-CPE electrodes toward Garnacha grapes collected weekly after véraison. the MONP selected. For instance, NiONP-CPEs were highly sensitive to phenols, whereas $\mathrm{TiO}_{2} \mathrm{NP}-\mathrm{CPEs}$ were sensitive to the acidity.

The chemical changes occurring during the ripening process could be followed using the MONP-based sensors. First, the intensity of the peaks increased, then intensity decreased and finally increased according with the tendency shown in Figure 2 where TPI values varied similarly. The irregular increase stopped when optimal ripeness was attained. Simultaneously, a decrease in the total acidity corresponded with a progressive decrease in the intensity of the peaks at negative potentials. As already stated in the introduction, multivariate methods have demonstrated to be a good strategy for the analysis of wines. It can be expected that they can also be useful to monitor the ripeness of grapes.

When PCA was carried out using the data obtained from the electronic tongue, it was observed that clusters corresponding to grapes collected in the first 4 weeks after véraison, were positioned in a counterclockwise organization "drawing" a circle (Figure 7A). In sampling number 5, the circle was closed. Surprisingly, in next samplings the positions were repeated and clusters corresponding to next specimens were situated continuing the counterclockwise trend following a "screw" structure. According to PCA, grapes could be considered matured when the clusters reached the original positions. Similar results have been obtained for all the grape varieties analyzed in this work. Similar results have also been observed in previous works where ETs have been used to monitor maturity of grapes (Medina-Plaza et al., 2016)

Figure 7B show the contribution of the variables in a loading plot. The loadings of each sensor (ten variables per sensor) spread along the four quadrants. This graph shows the high cross-selectivity achieved by the array of sensors.

Table 1 shows the statistical parameters obtained from the PLS-1 regression models (leave one out validation) correlating the output of the array of sensors with the chemical parameters (TPI, ${ }^{\circ}$ Brix, TA). PLS-1 builds regression models to establish a linear relationship between the set of predictors (X-variable: Voltammetric signals obtained from the array of sensors) and the

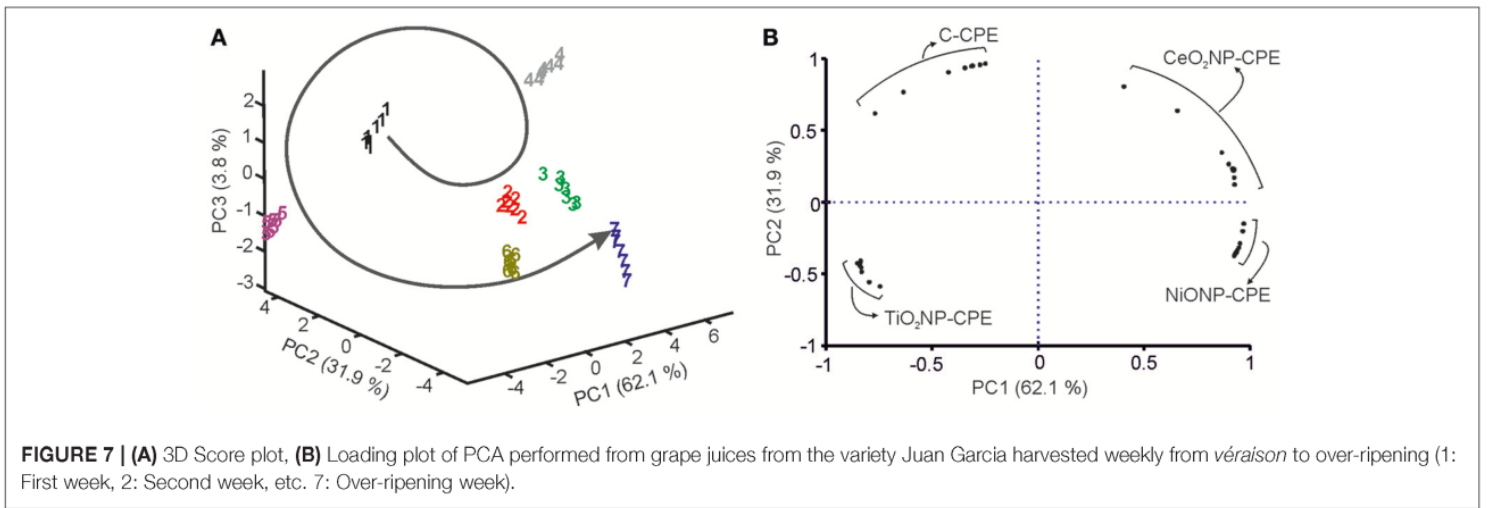




\begin{tabular}{|c|c|c|c|c|c|c|}
\hline Grape & Parameter & $R_{\mathrm{C}}^{2}$ (a) & $\operatorname{RMSE}_{C}(\mathbf{b})$ & $R_{\mathrm{p}}^{2}(\mathrm{c})$ & $\operatorname{RMSE}_{p}$ (d) & $\begin{array}{l}\text { Number of } \\
\text { components }\end{array}$ \\
\hline \multirow[t]{3}{*}{ Cabernet } & ${ }^{\circ}$ Brix & 0.87 & 0.86 & 0.85 & 0.95 & 3 \\
\hline & $\mathrm{TA}(g / L)$ & 0.87 & 0.81 & 0.81 & 1.02 & 7 \\
\hline & TPI & 0.86 & 2.13 & 0.83 & 2.42 & 2 \\
\hline \multirow[t]{3}{*}{ Garnacha } & ${ }^{\circ}$ Brix & 0.92 & 0.91 & 0.82 & 1.40 & 6 \\
\hline & $\mathrm{TA}(g / L)$ & 0.85 & 1.24 & 0.73 & 1.72 & 6 \\
\hline & TPI & 0.86 & 2.25 & 0.77 & 3.01 & 3 \\
\hline \multirow{3}{*}{$\begin{array}{l}\text { Juan } \\
\text { Garcia }\end{array}$} & ${ }^{\circ}$ Brix & 0.86 & 0.79 & 0.79 & 1.00 & 5 \\
\hline & $\mathrm{TA}(\mathrm{g} / \mathrm{L})$ & 0.80 & 1.08 & 0.70 & 1.39 & 6 \\
\hline & TPI & 0.84 & 1.54 & 0.79 & 1.84 & 4 \\
\hline \multirow{3}{*}{$\begin{array}{l}\text { Mencia } \\
\text { Pregadio }\end{array}$} & ${ }^{\circ}$ Brix & 0.97 & 0.22 & 0.96 & 0.26 & 5 \\
\hline & $T A(g / L)$ & 0.89 & 0.69 & 0.86 & 0.77 & 5 \\
\hline & TPI & 0.88 & 1.08 & 0.83 & 1.33 & 5 \\
\hline \multirow{3}{*}{$\begin{array}{l}\text { Mencia } \\
\text { Secano }\end{array}$} & ${ }^{\circ} \mathrm{Bnix}$ & 0.86 & 0.58 & 0.79 & 0.74 & 6 \\
\hline & $T A(g / L)$ & 0.81 & 0.84 & 0.71 & 1.06 & 6 \\
\hline & TPI & 0.86 & 1.57 & 0.82 & 1.79 & 3 \\
\hline \multirow{3}{*}{$\begin{array}{l}\text { Prieto } \\
\text { Picudo }\end{array}$} & ${ }^{\circ}$ Brix & 0.87 & 0.73 & 0.63 & 1.25 & 7 \\
\hline & $T A(g / L)$ & 0.88 & 0.93 & 0.70 & 1.52 & 7 \\
\hline & TPI & 0.83 & 1.33 & 0.81 & 1,43 & 3 \\
\hline \multirow[t]{3}{*}{ Rufete } & ${ }^{\circ} B n x$ & 0.85 & 0.49 & 0.83 & 0.53 & 3 \\
\hline & $T A(g / L)$ & 0.82 & 0.91 & 0.76 & 1.09 & 5 \\
\hline & TPI & 0.91 & 1.51 & 0.90 & 1.64 & 3 \\
\hline \multirow[t]{3}{*}{ Tempranillo } & ${ }^{\circ}$ Bnix & 0.81 & 0.89 & 0.76 & 1.01 & 6 \\
\hline & $T A(g / L)$ & 0.91 & 0.56 & 0.76 & 0.95 & 6 \\
\hline & TPI & 0.87 & 2.05 & 0.75 & 2.94 & 4 \\
\hline
\end{tabular}

Models were established for each variaty of grape considered separately. (a) Squarod corrokation cooficiont in callbration; (b) Aloot moan squaro orror of calibration; (c) Squared correlation coefficient in prediction; (d) Root mean squars emor of prediction.

set of responses (Y-variable: Chemical parameters obtained with traditional methods). PLS-1 finds the latent variables (indicated as number of components) in $\mathrm{X}$ that will best predict the latent variables in $\mathrm{Y}$.

In all varieties of grapes, good correlations were found with the Total Polyphenol Index. Using a low number of components, both the calibration and the prediction showed Squared Correlation Coefficients in calibration $\left(\mathrm{R}^{2} \mathrm{C}\right)$ and in prediction $\left(\mathrm{R}^{2} \mathrm{~V}\right)$ of ca. 0.8. Additionally, low Root Mean Square Error of calibration (RMSEC) and of prediction (RMSEp) were accomplished. These results confirm that the ET can be used to monitor the phenolic maturity of grapes. The ${ }^{\circ} \mathrm{Brix}$ and Total Acidity which are the parameters commonly used to evaluate the maturity of grapes, could also be predicted using the PLS 1 models but with lower correlation coefficients in prediction $(\sim 0.7)$ and the models need a higher number of components. In spite of the lower correlation, the models are good enough to confirm that the electrochemical responses also reflected the acidity and the sugar concentration. This indicates that it is possible to obtain simultaneous information of the three main parameters used to monitor the maturity of grapes.

\section{CONCLUSIONS}

The advantages of metal oxide nanoparticles as sensing materials for the detection of phenols has been evidenced. They rely on the excellent electrocatalytic properties of the metal oxide nanoparticles, that improved the sensitivity and limits of detection toward phenols with respect to unmodified sensors. Using these exceptional sensing properties, and the crossselectivity shown by different MONPs, an electronic tongue based on metal oxide nanoparticles has been developed. The chemical changes occurring during ripening could be followed using the MONP-based multisensor system. The sensors based on different MONP show an excellent degree of cross-selectivity. Using PLS 1 , correlations have been established between the signal of the multisensor system and the Total Polyphenol Index TPI, the ${ }^{\circ}$ Brix, and the Total Acidity.

According to this, simultaneous analysis of three important markers of maturity can be achieved using a multisensory system based on MONPs. This means that the ET can be a useful tool to establish with a better accuracy the optimal harvesting date in a single experiment. This method can be used as a supplementary tool to classical analytical techniques.

The results shown here demonstrate the excellent opportunities offered by metal oxide nanoparticles as sensing materials in electronic tongues.

\section{AUTHOR CONTRIBUTIONS}

MR-M: conceived the experiments an wrote the paper; $C G-H$ CM-P, CG-C, and YB: carried out the experiments; JF-E and EB-T: provided de grape samples and analyzed the grapes by chemical methods; MR-P and FM-P: carried out the chemometric analysis.

\section{ACKNOWLEDGMENTS}

Financial support by MINECO and FEDER (AGL2015-67482 R) and the Junta de Castilla y León FEDER (VA011U16) is gratefully acknowledged. CG-H thanks for the grant of JCYI (BOCYL-D-24112015-9).

\section{SUPPLEMENTARY MATERIAL}

The Supplementary Material for this article can be found online at: https://www.frontiersin.org/articles/10.3389/fchem. 2018.00131/full\#supplementary-material 


\section{REFERENCES}

Apetrei, C., Apetrei, I. M., De Saja, J. A., and Rodriguez-Méndez, M. L. (2011). Carbon paste electrodes made from different carbonaceous materials: application in the study of antioxidants. Sensors 11, 1328-1344. doi: $10.3390 /$ s110201328

Apetrei, I. M., Rodríguez-Méndez, M. L., Apetrei, C., Nevares, L, del Alamo, M. and de Saja. J. A. (2012). Monitoring of evolution during red wine aging in oak barrels and alternative method by means of an electronic panel test Food Res. Intnl. 45, 244-249. doi: 10.1016/j.foodres.2011.10.034

Boss, P. K., Bottcher, C., and Davies, C. (2014). Various influences of harvest date and fruit sugar content on different wine flavor and aroma compounds. Am. I. Enology Vitic. 65, 341-353. doi: 10.5344/ajev.2014.13137

Buratti, S., Ballabio, D., Giovanelli, G., Dominguez, C. M. Z., Moles, A., Benedetti, S., et al. (2011). Monitoring of alcoholic fermentation using near infrared and mid infrared spectroscopies combined with electronic nose and electronic tongue. Anal. Chim. Acta 697, 67-74. doi: 10.1016/j.aca.2011.04.020

Campbell, F. W., and Compton, R. G. (2010). The use of nanoparticles in electroanalysis: an updated review. Anal. Bioanal. Chem. 396, 241-259. doi: 10.1007/s00216-009-3063-7

Campos, I., Bataller, R., Armero, R., Gandia, J. M., Soto, J., Martínez-Mañez, R. et al. (2013). Monitoring grape ripeness using a voltammetric electronic tongue. Food Res. Int. 54, 1369-1375. doi: 10.1016/j.foodres.2013.10.01

Cetó, X., González-Calabuig, A., Crespo, N., Pérez, S., Capdevila, J., Puig-Pujol, A. et al. (2017). Electronic tongues to assess wine sensory descriptors. Talanta 162 218-224 doi: 10.1016/j.talanta 2016.09 .055

Codinachs, L M. L., Kloock, J. P., Schőning, M. J., Baldi, A., Ipatov, A., Bratov, A. et al. (2008). Electronic integrated multisensor tongue applied to grape juice and wine analysis. Analyst 113, 1440-1448. doi: 10.1039/b801228h

Cosio, M. E., Benedetti, S., and Scampio, M. (2012). Electronic Noses and Tongues in Chemical Analysis of Food: Techniques and Applications. London: Academic Press.

Cozzolino, D., MoCarthy, J., and Bartowsky, E. (2012). Comparison of near infrared and mid infrared spectroscopy to discriminate between wines produced by different Oenococcus oeni strains after malolactic fermentation: feasibility study. Food Contro! 26, 81-87, doi: 10.1016/jfoodcont 2012.01003

Godelmann, R., Fang, F., Humpfer, E., Schūtz, B., Bansbach, M., Schăfer, H. et al. (2014). Targeted and nontargeted wine analysis by $1 \mathrm{H}$ NMR spectroscopy combined with multivariate statistical analysis. Differentiation of importan parameters: Grape variety, geographical origin, year of vintage. J. Agric. Food Chem. 61, 5610-5619, doi: 10.1021/j4400800d

Gutiérrez, J. M., Haddi, Z., Amari, A.,Bouchikhi, B., Mimendia, A., Cetó, X., et al. (2013). Hybrid electronic tongue based on multisensor dat fusion for discrimination of beers Sens. Actuators B: Chem. 177, 989-996. doi: $10.1016 /$ j.snb.2012.11.110

Gutiérrez, I. M., Moreno-Barón. L. Pividori, M. I. Alegret, S. and Del Valle, M. (2010). A voltammetric electronic tongue made of modified epoxy-graphite electrodes for the qualitative analysis of wine. Microchim. Acta 169, 261-268. doi: $10.1007 / \mathrm{s} 00604-010-0351-\mathrm{z}$

Gutiérrez, M., Domingo, C., Vila-Planas, I., Ipatov, A., Capdevila, F., Demming S., et al. (2011). Hybrid electronic tongue for the characterization and quantification of grape variety in red wines. Sens. Actuators B Chem. 153 695-702. doi: 10.1016/j.snb.2011.02.020

Kilmartin, P. A., Zou, H. L, and Waterhouse, A. L. (2001). A cyclic voltammetry method suitable for characterizing antioxidant properties of wine and wine phenolics. J. Agric. Food Chem. 49, 1957-1965. doi: 10.1021/if001044u

Kirsanov, D., Mednova, O., Vietoris, V., Kilmartin, P. A., and Legin, A. (2012). Towards reliable estimation of an electronic tongue predictive ability from PLS regression models in wine analysis. Talanta 90, 109-116. doi: $10.1016 /$ j.talanta.2012.01.010

Lin, X. Y., Ni, Y. N., and Kokot, S. (2013). Glassy carbon electrodes modified with gold nanoparticles for the simultaneous determination of three food antioxidants. Anal. Chim. Acta 765, 54-62. doi: 10.1016/j.aca.2012.12.036

Lvova, L, Kirsanov, D., Di Natale, C., and Legin, A. (2014). Multisensor systems for chemical analysis: Materials and Sensors. Boca Ratón. FL C CRC Press.

Medina-Plaza, C., De Saja, J. A., Fernández-Escudero, I. A., Barajas-Tola, E. Medrano, G., et al. (2016). Array of biosensors for discrimination of grapes according to grapevariety, vintage and ripeness. Anal. Chim. Acta 947,16-22 doi: 10.1016/j.aca 2016.10 .032
Medina-Plaza, C., De Saja, J. A. and Rodríguez-Méndez, M. L. (2014a). Bioelectronic tongue based on lipidic nanostructured layers containing phenol oxidases and lutetium bisphthalocyanine for the analysis of grapes. Biosen Bioelectron. 57, 276-283. doi: 10.1016/j.bios.2014.02.023

Medina-Plaza, C., Garcia-Cabezón, C., Garcia-Hernández, C., Bramorski, C. and Blanco-Val, Y., Martin-Pedrosa, et al. (2015a). Analysis of organic acids and phenols of interest in the wine industry using Langmuir-Blodget films based on functionalized nanoparticles. Anal. Chim. Acta 853, 572-578. doi: 10.1016/j.aca.2014.10.046

Medina-Plaza, C., Revilla, G., Munoz, R., Fernández-Escudero, I. A., Barajas, E., Medrano, G., et al. (2014b). Electronic tongue formed by sensors and biosensors containing phthalocyanines as electron mediators. Application to the analysis of red grapes. J. Porphyrins Phthatocyanines 18, 76-86. doi: $10.1142 / \$ 1088424613501137$

Medina-Plaza, C., Rodríguez-Méndez, M. L., Sutter, P., Tong, X., and Sutter, E. (2015b). Nanoscale Au-In alloy-oxide core-shell particles as electrocatalysts for efficient hydroquinone detection. J. Phys. Chem. C 119, 25100-25107. doi: 10.1021/acs.jpcc.5b07960

Meléndez, E., Ortiz, M. C., Sarabia, L. A., and fñiguez, M., Puras, P. (2013). Modelling phenolic and technological maturities of grapes by means of the multivariate relation between organoleptic and physicochemical properties. Anal. Chim. Acta 761, 53-61. doi: 10.1016/..aca.2012.11.021

Mercante, L A., Scagion, V. P., Pavinatto, A., Sanfelice, R. C., Mattoso, L. H. C. and Correa. D. S. (2015). Electronic tongue based on nanostructured hybrid films of gold nanoparticles and phthalocyanines for milk analysis. J. Nanomat. 16:402, doi: $10.1155 / 2015 / 890637$

Nogales-Bueno, J., Hernández-Hierro, J. M., Rodriguez-Pulido, F. J., and Heredia, F. J. (2014). Determination of technological maturity of grapes and total phenolic compounds of grape skins in red and white cultivars during ripening by near infrared hyperspectral image: a preliminary approach. Food Chem. 152. 586-591. doi: $10.1016 /$ j. foodchem. 2013.12 .030

OIV (2013). Compenditu of International Methods of Analysis of Wines and Musts. Organisation Internationale de la Vigne et du Vin. Paris.

Prieto, N., Gay, M., Vidal, S., Aagaard, O., De Saja, J. A., and Rodríguez-Méndez, M. L (2011). Analysis of the influence of the type of closure in the organoleptic characteristics of a red wine by using an electronic panel. Food Chem. 129. 589-594. doi: 10.1016/j.foodchem.2011.04.071

Rodriguez-Méndez, M. L. (2016). Electronic Noses and Tongues in Food Industry. London: Academic Press.

Rudnitskaya, A., Schmidtke, L. M., Reis, A., Domingues, M. R. M., Delgadillo, I. Debus, B., et al. (2017). Measurements of the effects of wine maceration with oak chips using an electronic tongue. Food Chem. 229, 20-27. doi: 10.1016/.foodchem.2017.02.013

Saha, K., Agasti, S. S., Kim, C., Li, X., and Rotello, V. M. (2012). Gold nanoparticles in chemical and biological sensing. Chem. Rev. 112, 2739-2779. doi: $10.1021 / \mathrm{cr} 2001178$

Sharma, S., Kori, S., and Parmar, A. (2015). Surfactant mediated extraction of tota phenolic contents (TPC) and antioxidants from fruits juices. Food Chem. 185. 284-288. doi: 10.1016/j.foodchem.2015.03.106

Sharpe, E, Bradley, R. Frasco, T, Jayathilaka, D, Marsh, A, and Andreescu, S. (2014). Metal oxide based multisensor array and portable database for field analysis of antioxidants. Sens. Actuators B Chem. 193, 552-562. doi: 10.1016/j.snb.2013.11.088

Xu, Y. P., Simon, J. E., Welch, C., Wightman, I. D., Ferruzzi, M. G., Ho, L. et al. (2011). Survey of polyphenol constituents in grapes and grape-derived products. J. Agric. Food Chem. 59, 10586-10593. doi: 10.1021/jf202438d

Conflict of Interest Statement: The authors declare that the research was conducted in the absence of any commercial or financial relationships that could be construed as a potential conflict of interest.

Copyright (c) 2018 Garcia-Hernander, Medina-Plaza, Garcia-Cabezon, Blanco, Fernandez-Escudero, Barajas-Tola, Rodnguez-Perez, Martin-Pedrosa and Rodriguez-Mendez. This is an open-access article distributed under the terms of the Creative Commons Attribution License $(\triangle C B Y)$. The use, distribution or reproduction in other forums is permitted, provided the original author(s) and the copyright owner are credited and that the original publication in this joumal is cited. in accordance with accepted academic practice. No use, distribution or reproduction is permitted which does not comply with these terms. 


\subsection{Paper III}

A different approach for the analysis of grapes: Using the skin as sensing element.

By Raquel Muñoz, Celia García-Hernández, Cristina Medina-Plaza, Cristina García-Cabezón, J.A. Fernández-Escudero, Enrique Barajas, Germán Medrano and María Luz Rodríguez-Méndez.

Food Research International 107 (2018) 544-550.

(doi: 10.1016/j.foodres.2018.02.060) 


\subsubsection{Motivation and objective}

Phenolic compounds (phenolic acids, flavonoids and tannins) are important components of wines that can strongly influence their final organoleptic properties. In fact, the characteristic color in red wines is, to a large extent, ascribable to the phenolic substances present in the grape skin cells, which are transferred to the must during the maceration step. The optimal point of grape harvesting is traditionally indicated by measuring the sugar content and acidity of the grapes. However, the phenolic content is a factor that must be considered to obtain wines with a high quality.

Grape skins contain phenols bounded to polysaccharides by means of hydrophobic interactions and hydrogen bonds (called cell-wall phenols), and phenols inside the vacuoles of the plant cells or associated with the cell nucleus (called non-cell-wall phenols). The phenolic composition of wines will determine such factors as color, sensory characteristics and the healthy quality of wines. The phenols are transferred from the grapes into the must, so the wine depends on how those phenols are bound in the grape skins. During the maceration step in the winemaking process, the phenolic compounds are transferred from the grape skins to the must.

The polyphenolic and antioxidant content in food and beverages can be assessed by recognized traditional spectrophotometric methods, such as Total Polyphenol index (TPI), FRAP, DPPH, ORAC and/or Folin-Ciocalteu Indexes, which are methods based on the evaluation of the capabilities of an oxidizing agent to induce oxidative damage to a substrate, and also by using multi-parametric methods such as infrared spectroscopy (FTIR, NIR) or gas and liquid chromatography (HPLC) combined with chemometric tools. However, electronic tongue technology is emerging as a supplementary or alternative method to assess food quality. In the case of grapes and wines, as phenolic compounds are redox species, they can be analyzed using electrochemical methods by means of electronic tongues. Unlike the spectroscopy methods, ETs can help to improve the sensitivity at a relatively low cost.

The aim of this work is to develop a new and alternative voltammetric sensor based on a carbon electrode covered with the skin of grapes to monitor their phenolic maturity by improving the intimate contact between the analyte and the electrode surface so that there is no need for sensing material to enhance the electron transfer. By using PLS-1, correlation models were 
performed between the voltammetric responses obtained with the skin-modified sensors and the phenolic (Glories or TPI) and sugar ('Brix, density, sugar content) parameters.

\subsubsection{Summary of the research work}

Carbon paste electrodes were prepared by mixing graphite powder with a mineral oil (nujol) as already mentioned. The carbon paste was put inside a plastic syringe and a copper wire was used as electrical contact. Then, a piece of grape skin was carefully placed on the surface of the CPE and fixed with a rubber O-ring, maintaining the inner side of the skin in contact with the carbon paste. The sensors were prepared using 3 grape varieties: Prieto Picudo, Mencía (Regadío) and Juan García, collected weekly from véraison to harvesting date. In order to evaluate the repeatability and reproducibility of the skin-carbon paste-based sensors (S-CPE), 3 sensors were prepared for each sample, using 3 different grapes from each bunch, making a total of 9 electrodes for each week and 45 electrodes for the whole experiment (9 S-CPE sensors $\times 5$ weeks).

Grapes samples were provided by the vineyard of the Instituto Tecnológico Agrario de Castilla y León (ITACyL). All samples were collected weekly from véraison to harvesting date. The Oenological Centre of Castilla y León provided the chemical parameters of TPI, Brix degree, density, sugar content, degree 16.8 and degree 17.5. In addition, the Glories method was carried out to evaluate the phenolic maturity of the grapes at $\mathrm{pH} 1$, providing information of total potential in anthocyanins, and at $\mathrm{pH} 3$, giving information of the total potential in extractible anthocyanins at the $\mathrm{pH}$ of the wine.

Once the S-CPE sensors had been prepared, they were tested in phosphate buffer to evaluate their electrochemical responses. Voltammetric responses showed anodic peaks in the range from 0.5 to $0.7 \mathrm{~V}$ and cathodic peaks ranging from -0.5 to $-0.8 \mathrm{~V}$. These peaks were related to the phenols present in the skin, mainly anthocyanins and proanthocyanins. The electrochemical response for each grape showed that each grape skin has a specific phenolic composition. It is important to notice that the structure and mechanical properties of each type of skin, such as porosity, thickness or hardness, might also influence the electrochemical responses, giving a distinctive response for each grape. Thus, grape skins were monitored in phosphate buffer from week 1 (just one week after véraison) to week 5 (harvesting date). The variations in skin composition during ripening were observed. The main characteristic was 
observed in the intensity and position of the peaks related to phenols in the collected voltammograms. Peaks increased in intensity from week 1 until a maximum was attained just before harvesting time, after which the intensities decreased. This result may be associated with the fact that the level of anthocyanins increases during ripening and decreases slightly in ripened grapes. Shifts in the position of the cathodic and anodic waves are usually related to polymerization processes. The Mencía sample was the slowest to reach phenolic skin maturation, showing the highest intensity in the week 4, whereas the Prieto Picudo and Juan García ripened faster, with a maximum intensity in week 3. Comparing these results with such traditional methods as the Glories method and TPI, in which the evaluation of phenolic content includes polyphenols extracted from the whole grape (skin, seeds and flesh), the results showed a similar trend to that observed in the Glories results at $\mathrm{pH} 3.0$ and at $\mathrm{pH} 1.0$. However, the differences were more easily observable using S-CPE sensors than the Glories method, while the decrease observed prior to maturation can be detected more easily. TPI values did not show any inflexion and were mainly stable from week 1 to week 4 , suddenly increasing in the harvesting week. Therefore, using S-CPE, we can predict the harvesting date one or two weeks before the date by observing the inflexion point in the electrochemical responses.

Using PCA analysis, we obtained information about the ripening over weeks. The PCA loading plots from weeks 1,3 and 5 showed a progressive change in the places where the samples were located. In week 1 samples, Prieto Picudo and Mencía appeared to overlap and were separate from Juan García, whereas in week 2, the Mencía and Juan García samples appeared to overlap and were separate from the Prieto Picudo. However, in week 5, each sample appeared to be located in a different region in the diagram, demonstrating that the phenolic maturity had been reached and that each grape shows a specific, characteristic phenolic composition.

The progressive maturing of the grapes was analyzed by means of partial least squaresdiscrimination analysis (PLS-DA). It was observed that the first Principal Component shifted to negative values as the ripening advanced. Then, in week 5 , the PC1 returned to positive values, illustrating a circular behavior. This trend has already been described using multisensor systems based on metal oxide nanoparticle CPEs.

Finally, using PLS-1 regression models, we established linear correlations between the data obtained weekly using S-CPE and the chemical parameters obtained by classical methods. 
Since voltammograms contain information not only about polyphenols, but also about other components present in the skin, such as sugars, acids or ions, the correlations were performed for each chemical parameter. The Juan García and Prieto Picudo skin sensors showed a good correlation with Glories at pH 1, considering the total extractable polyphenols present in the skin, whereas at pH 3, only the easily extractable polyphenols were considered. The Juan García sensors also had a good correlation with the TPI; however, this result was isolated and the correlations with the rest of the parameters could be established, but with low correlation coefficients (lower than 0.70) and high latent variables. In the case of the Mencía skin sensors, no correlations could be established. These results are, to a large extent, determined by the different properties of the skins, such as porosity or hardness, which can affect the extractability of phenol compounds and may be due to environmental factors. For instance, in this study, we found it difficult to separate the skin from the flesh in the Mencía grape variety. In this sense, the degree of adhesion between skin and flesh during ripening was more variable than for the Juan García and Prieto Picudo varieties, where the skin and flesh were properly separated in every sampling. Moreover, in week 3, the Mencía grapes were collected after heavy rain, and skin and flesh could hardly be separated. These facts might have interfered with the permeability of the skin and therefore with the diffusion of the ions and electron transfer from the skin to the electrode surface. 



\section{A different approach for the analysis of grapes: Using the skin as sensing element}

Raquel Muñoz ${ }^{\mathrm{a}, \mathrm{b}}$, Celia García-Hernández ${ }^{\mathrm{a}}$, Cristina Medina-Plaza ${ }^{\mathrm{a}}$, Cristina García-Cabezón ${ }^{\mathrm{a}}$, J.A. Fernández-Escudero ${ }^{\mathrm{c}}$, Enrique Barajas ${ }^{\mathrm{d}}$, Germán Medrano ${ }^{\mathrm{e}}$, María Luz Rodriguez-Méndez ${ }^{\mathrm{a}, *}$

${ }^{\text {a } G r o u p ~ o f ~ S e n s o r s ~ U V A S E N S, ~ U n i v e r s i d a d ~ d e ~ V a l l a d o l i d, ~} 47011$ Valladolid, Spain

b Dept. Bioquímica, Biologia Molecular y Fisiologí, Universidad de Valladolid, 47011 Valladolid, Spain

${ }^{c}$ Estación Enológica de Castilla y León, C/Santísimo Cristo, 26, 47490 Rueda, Valladolid, Spain

d ITACYL Avenida de Burgos, KM.118, Finca Zamadueñas, 47071 Valladolid, Spain

${ }^{e} R \& D$ Dept. Bodega Cooperativa de Cigales, C/Las Bodegas, $s / n, 47270$ Cigales, Valladolid, Spain

\section{A R T I C L E I N F O}

\section{Keywords:}

Phenolic maturity

Grape

Voltammetric sensor

Skin-sensor

TPI

Multisensor systems

\begin{abstract}
A B S T R A C T
In this work, an alternative method to monitor the phenolic maturity of grapes was developed. In this approach, the skins of grapes were used to cover the surface of carbon paste electrodes and the voltammetric signals obtained with the skin-modified sensors were used to obtain information about the phenolic content of the skins. These sensors could easily detect differences in the phenolic composition of different Spanish varieties of grapes (Mencía, Prieto Picudo and Juan García). Moreover, sensors were able to monitor changes in the phenolic content throughout the ripening process from véraison until harvest.

Using PLS-1 (Partial Least Squares), correlations were established between the voltammetric signals registered with the skin-modified sensors and the phenolic content measured by classical methods (Glories or Total Polyphenol Index). PLS-1 models provided additional information about Brix degree, density or sugar content, which usually used to establish the harvesting date. The quality of the correlations was influenced by the maturation process and the structural and mechanical skin properties. Thus the skin sensors fabricated with Juan García and Prieto Picudo grapes (that showed faster polyphenolic maturation and a higher amount of extractable polyphenols than Mencía), showed good correlations and therefore could be used to monitor the ripening.
\end{abstract}

\section{Introduction}

Harvesting grapes in the optimal point of maturity is a main concern for winemakers (Jackson, 2014). It is therefore important to develop chemical markers to define the optimal ripeness. The physicochemical and biochemical processes occurring during ripening process, produce a continuous rising of the sugars concentration and a decrease in the acid levels. For this reason, the classical parameters measured to monitor grape ripeness and to determine the harvesting date are the berry weight, the must density and the relation between sugars content and total acidity (Robredo, Junquera, González San José, \& Barren, 1991) in musts.

To elaborate a high quality red wine, grapes need to reach an appropriate phenolic maturation. To elaborate a high quality red wine, grapes must reach an appropriate phenolic maturation. According to the literature, phenols accumulate in the berries during ripening.
Therefore, an increase in the total phenolic content is a good indicator of the grape maturity (González-San José, Barren, \& Díez, 1990; González-San José, Barren, Junquera, \& Robredo, 1991; Mahmood, Anwar, Abbas, \& Saari, 2012).

The phenolic maturity has been measured using different methods and TPI is one possible approach (Adams, 2006; Garrido \& Borges, 2013; Kennedy, 2008; Nogales-Bueno et al., 2017).

In spite of the variety of methods mentioned, the assessment of the grape maturity is difficult task because each parameter changes with ripeness in a different manner (since they depend on different biochemical pathways). Moreover, the optimal maturity values at harvest depend on the variety of grape, the type of wine being made, weather or seasonal factors, or viticultural practices among many other factors (Lohitnavy, Bastian, \& Collins, 2010). Another difficulty is that the active metabolism of the skin during grape development has an essential effect on the final quality of the grape berries and wine (Negri

Abbreviations: CPE, carbon paste electrode; PCA, Principal Component Analysis; PLS-1, Partial Least Squares; TPI, Total Polyphenol Index; S-CPE, Skin-CPE; PC, Principal Component; PLS-DA, Partial Least Squares Discriminant Analysis

* Corresponding author at: Dpt. Inorganic Chemistry, Escuela de Ingenierías Industriales, Paseo del Cauce, 59, 47011 Valladolid, Spain.

E-mail address: mluz@eii.uva.es (M.L. Rodriguez-Méndez).

https://doi.org/10.1016/j.foodres.2018.02.060

Received 18 December 2017; Received in revised form 16 February 2018; Accepted 25 February 2018

Available online 02 March 2018

0963-9969/ @ 2018 Elsevier Ltd. All rights reserved. 
Paper III: A different approach for the analysis of grapes: Using the skin as sensing element.

Table 1

Results of the chemical analysis carried out by traditional chemical methods.

\begin{tabular}{|c|c|c|c|c|c|c|c|c|c|}
\hline Sample & Week & $\begin{array}{l}\text { Glories } \\
\text { pH } 3\end{array}$ & $\begin{array}{l}\text { Glories } \\
\mathrm{pH} 1\end{array}$ & TPI & Brix & $\begin{array}{l}\text { Density } \\
(\mathrm{g} / \mathrm{mL})\end{array}$ & $\begin{array}{l}\text { Sugars } \\
(\mathrm{g} / \mathrm{L})\end{array}$ & $\begin{array}{l}\text { Degree } \\
16.8\end{array}$ & $\begin{array}{l}\text { Degree } \\
17.5\end{array}$ \\
\hline \multirow[t]{5}{*}{ Mencía } & S1 & 0.255 & 0.396 & 11 & 18.71 & 1.0760 & 177.2 & 10.53 & 10.15 \\
\hline & S2 & 0.340 & 0.573 & 11 & 20.16 & 1.0822 & 193.1 & 11.47 & 11.05 \\
\hline & s3 & 0.454 & 0.579 & 11 & 21.65 & 1.0889 & 210.3 & 12.49 & 12.00 \\
\hline & $\$ 4$ & 0.394 & 0.508 & 9 & 20.58 & 1.0840 & 197.7 & 11.75 & 11.30 \\
\hline & S5 & 0.244 & 0.452 & 17 & 20.99 & 1.0857 & 202.2 & 12.01 & 11.55 \\
\hline \multirow[t]{5}{*}{ Priero Plcudo } & s1 & 0.322 & 0.376 & 9 & 19.56 & 1.0795 & 186.3 & 11.07 & 10.65 \\
\hline & S2 & 0.474 & 0.586 & 9 & 20.93 & 1.0857 & 202.2 & 12.01 & 11.55 \\
\hline & s3 & 0.443 & 0.824 & 11 & 22.65 & 1.0933 & 221.7 & 13.17 & 12.70 \\
\hline & 54 & 0.357 & 0.662 & 9 & 21.05 & 1.0862 & 203.3 & 12.08 & 11.60 \\
\hline & \$5 & 0.341 & 0.523 & 19 & 22.89 & 1.0943 & 224.1 & 13.31 & 12.80 \\
\hline \multirow[t]{5}{*}{ Juan Garcta } & s1 & 0.361 & 0.396 & 14 & 17.30 & 1.0699 & 161.5 & 9.59 & 9.25 \\
\hline & s2 & 0.376 & 0.480 & 14 & 19.80 & 1.0809 & 189.7 & 11.27 & 10.80 \\
\hline & s3 & 0.593 & 0.609 & 18 & 21.59 & 1.0884 & 209.1 & 12.42 & 11.95 \\
\hline & S4 & 0.458 & 0.583 & 17 & 20.70 & 1.0849 & 200.0 & 11.88 & 11.45 \\
\hline & S5 & 0.316 & 0.418 & 29 & 22.18 & 1.0911 & 216.0 & 12.83 & 12.35 \\
\hline
\end{tabular}

et al., 2008; Rockenbach et al., 2011; Sokolowsky, Rosenberger, \& Fischer, 2015). In fact, the majority of the phenolic compounds present in wine are derived from skin which contains anthocyanins, flavonols and proanthocyanidins (tannins) (Jara-Palacios, Hernanz, EscuderoGilete, \& Heredia, 2014; Nogales-Bueno et al., 2017). So, methods are needed that analyze the phenolic composition of skins. Phenolic compounds are redox active compounds that can be analyzed by means of electrochemical methods (Blasco, González-Crevillén, González, \& Escarpa, 2007; Hoyos-Arbelaez, Vazquez, \& Contreras-Calderon, 2017; Kilmartin, 2013; Kirsanov, Mednova, Vietoris, Kilmartin, \& Legin, 2012; Rudnitskaya et al., 2017). For instance, using simple electrodes (graphite, platinum or ITO glass) the antioxidants present in teas (Buratti, Scampicchio, Giovanelli, \& Mannino, 2008; Piljac-Žegarac, Valek, Stipčević, \& Martínez, 2010), natural juices (Bordonaba \& Terry, 2012; Pisoschi, Cheregi, \& Danet, 2009), wines (Makhotkina \& Kilmartin, 2010), or onions (Zielinska, Wiczkowski, \& Piskula, 2008) can be analyzed.

Chemically modified electrodes can also be used for the detection of antioxidants. In such sensors, the electrode surface is covered with sensing material that facilitates the charge-transfer reactions between the electrode and the solution (Barroso, Santos-Alvarez, Delerue-Matos, \& Oliveira, 2011; Durst, Baumner, Murray, Buck, \& Andrieux, 1997; Ziyatdinova, Kozlova, \& Budnikov, 2016). Many sensing materials have been used as chemical modifiers, for instance, carbon nanotubes, graphene, nanoparticles, porphyrins, phthalocyanines or conducting polymers among others (García-Hernández, García-Cabezón, MartínPedrosa, de Saja, \& Rodríguez-Méndez, 2016).

Electrochemical sensors can be combined to form Electronic tongues (ET) According to the IUPAC, an electronic tongue is a multisensor system, which consists of a number of low-selective sensors and crosssensitivity to different species in solution, and an appropriate method of pattern recognition and/or multivariate calibration for data processing (Rodriguez-Méndez, 2016). Such systems provide global information about complex samples. For instance, the electrochemical responses of the multisensor system, contain information not only of the electroactive components of the sample (i.e. phenols) but also, about other components such as protons, ions, etc. The responses of the sensors are analyzed using a pattern recognition software that allows discriminating samples with different characteristics. In addition, after an appropriate training, mathematical models can be built to correlate the measures carried out using the multisensor system and measures carried out by traditional chemical analysis.

Inspired by the concept of ETs, he aim of this work is to develop a new method to monitor the phenolic maturity of grapes based on Carbon Paste Electrodes (CPEs) that were modified with a piece of the skin of the corresponding grape. Three different varieties of Vitis vinifera grapes were included in this study (Mencía, Prieto Picudo and Juan Garcia). Grape samples were collected weekly along their ripeness process, starting one week after véraison and until complete maturity. The electrochemical response of the skin-sensor (S-CPE) was registered in phosphate buffer (pH7.0) using cyclic voltammetry. The discrimination capability of the electrodes and the ability to monitor the phenolic ripening, were analyzed by means of Principal Component Analysis (PCA) and Partial Least Squares (PLS-1). In addition, the electrochemical response of the electrodes was analyzed and compared with chemical data measured by classical chemical methods.

\section{Materials and methods}

\subsection{Reagents and solutions}

All reagents were of high purity and purchased from Sigma-Aldrich. Solutions were prepared using deionized water (Milli-Q, Millipore). Phosphate buffer solution $0.01 \mathrm{M}$ ( $\mathrm{pH} 7.0$ ) was prepared from potassium monobasic and dibasic phosphate salts.

\subsection{Grape and must samples: selection and chemical analysis}

Samples of three different varieties of red grapes: Mencía, Prieto Picudo and Juan Garcia were harvested in September-October 2013 in the vineyards of the Bodega Cooperativa de Cigales and of the Instituto Tecnológico Agrario de Castilla y León, both located in the Castilla y León region (Spain). 200 berries of each variety were collected on a weekly basis, starting one week after véraison until harvest $(12,20$ and 26 of September, and 4, 8 of October 2013, samples numbered S1 to S5 respectively).

The polyphenolic content of the grapes was estimated using Total Polyphenol Index (TPI) measured as the Absorbance at $280 \mathrm{~nm}$ and the Glories method (Glories \& Augustin, 1993). Brix, Density, sugar content, Degree (16.8) and Degree (17.5) were analyzed following the international regulations (OIV, 2013). All chemical parameters are shown in Table 1.

\subsection{Skin-carbon paste electrodes (S-CPE)}

Carbon paste electrodes were prepared by mixing graphite powder (High purity Ultracarbon", Ultra F purity. Bay City, MI, USA) with the mineral oil Nujol (Fluka), in 1.5:1 (w/w) ratio. The obtained paste was packed in a $1 \mathrm{~mL}$ PVC (polyvinyl chloride) syringe (Yang, Denno, Pyakurel, \& Venton, 2015). A copper wire, inserted into the syringe, was used as the electrical contact. The voltammetric behavior of the carbon paste electrodes was analyzed in $0.01 \mathrm{M}$ phosphate buffer 
Table 2

Skin-CPE sensors prepared in this work.

\begin{tabular}{llll}
\hline Sampling date & Mencía & Prieto Picudo & Juan García \\
\hline September 12, 2013 & S1-M-CPE & S1-PP-CPE & S1-JG-CPE \\
September 20, 2013 & S2-M-CPE & S2-PP-CPE & S2-JG-CPE \\
September 26, 2013 & S3-M-CPE & S3-PP-CPE & S3-JG-CPE \\
October 4, 2013 & S4-M-CPE & S4-PP-CPE & S4-JG-CPE \\
October 8, 2013 & S5-M-CPE & S5-PP-CPE & S5-JG-CPE \\
\hline
\end{tabular}

adjusted to $\mathrm{pH} 7.0$.

A piece from the skin of a fresh grape was carefully peeled using a cutter, placed on the surface of the CPE and fixed with a rubber O-ring. It is important to mention that the inner side of the skin was placed in contact with the carbon paste. Sensors were prepared using grapes from three different varieties that were collected periodically (S1 to S5). Sensors prepared are collected in Table 2.

In order to evaluate the repeatability and reproducibility of the method, devices were prepared using three different grapes from each bunch, choosing those grapes representative of the general ripening state of the vineyard. Moreover, three pieces of skin were cut from each grape and used to prepare electrodes. Using this method, nine electrodes ( 3 grapes $\times 3$ pieces of skin) were prepared from each type of grape.

\subsection{Electrochemical measurements}

Cyclic voltammetry was performed using a three electrode configuration, in an EG\&G PARC Model 263 potentiostat/galvanostat (Princeton Applied Research Corp., NJ, USA) controlled by the Echem software. $S$-CPEs were used as the working electrode and the counter electrode was a platinum wire. Potential values were referred to an $\mathrm{Ag}$ / $\mathrm{AgCl}$ electrode. All measurements were carried out in $20 \mathrm{ml}$ of $0.01 \mathrm{M}$ phosphate buffer adjusted to pH7.0. Cyclic voltammograms were registered from -1.1 to $+1.1 \mathrm{~V}$ (the scan started at $0 \mathrm{~V}$ ) at a sweep rate of $0.1 \mathrm{~V} / \mathrm{s}$. Voltammograms shown in this work correspond to the average of nine replicas of each grape sample.

\subsection{Statistical analysis}

Principal Component Analysis (PCA) and Partial Least SquaresDiscriminant Analysis (PLS-DA), a non-supervised and supervised multivariate method respectively, were used to analyze and compare the voltammetric results of the different samples along maturation. Mathematical correlations between the signals obtained using the sensors and data obtained by classical chemical analysis were established using PLS-1 models.

In order to reduce the number of variables used in multivariate analysis, feature extraction was carried out using the kernel method to compress the information from the original signals and to extract meaningful data from the readings (Medina-Plaza et al., 2015). Using this method, voltammograms were multiplied by 10 smooth, bellshaped windowing functions defined as:

$$
\mathrm{K}_{\mathrm{i}}\left(\mathrm{V}_{\mathrm{j}}\right)=\frac{1}{1+\left(\frac{\mathrm{V}_{\mathrm{j}}-\mathrm{c}_{\mathrm{i}}}{\mathrm{a}_{\mathrm{i}}}\right)^{2 \mathrm{~b}_{\mathrm{i}}}}
$$

where $a_{i}, b_{i}$ and $c_{i}$ define the width, shape and center of the different windowing functions $\mathrm{K}_{\mathrm{i}}$. Subsequently, data were integrated with respect to voltage. Thus, each curve provided ten variables which were used as the input data source for statistical analysis. The analysis was carried out using the software Matlab v5.3 (The Mathworks Inc., Natick, MA, USA).

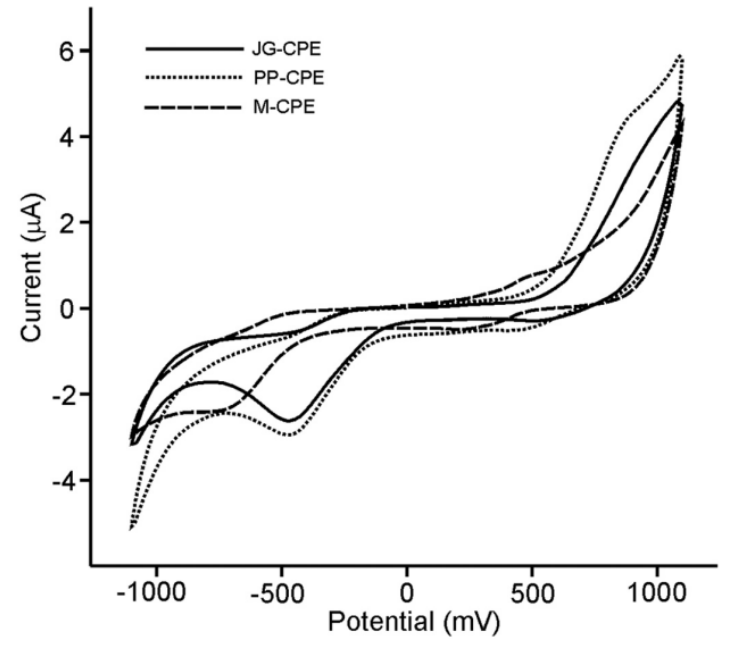

Fig. 1. Cyclic voltammetry of electrodes immersed in $0.01 \mathrm{M}$ phosphate buffer $\mathrm{pH} 7.0$. Scan rate $0.1 \mathrm{~V} / \mathrm{s}$. The grapes correspond to the second sampling on 20th September.

\section{Results and discussion}

As described in the Experimental section, a new class of sensors dedicated to the analysis of grapes was developed. Pieces of the skin grape were cut and placed on the top of the carbon surface of a CPE electrode to obtain Skin-CPE ( $S$-CPE) sensors.

The voltammetric behavior of $S$-CPE sensors modified with the skin of grapes obtained from three varieties Mencía, Prieto Picudo and Juan García (denoted as M-CPE, PP-CPE and JG-CPE) was tested in $0.01 \mathrm{M}$ phosphate buffer $\mathrm{pH} 7.0$ in the potential range -1.1 to $+1.1 \mathrm{~V}$. Nine replicas of each sensor were measured to study the repeatability of the voltammograms registered. Fig. 1 illustrates the electrochemical responses of $S$-CPEs modified with skins of the three varieties of grapes. In this example, grapes were collected the same day (corresponding to the second sampling collected fifteen days after véraison). Voltammograms showed an anodic wave at ca. 0.5 to $0.7 \mathrm{~V}$ and a cathodic wave in the region -0.5 to $-0.8 \mathrm{~V}$. According to the literature, these peaks can be associated to phenols present in grapes which are mainly anthocyanins and proanthocyanidins (Apetrei, Apetrei, de Saja, \& Rodriguez-Méndez, 2011; Blasco et al., 2007; Kilmartin, 2013; Makhotkina \& Kilmartin, 2013; Rodriguez-Mendez et al., 2014). Moreover, the position of redox peaks observed in diluted wines carried out using carbon electrodes, depends on the nature of the phenols present in the solution. In our case, due to the complexity of the electrode surface and the variety of phenols present on the skin, redox peaks were wider than those observed in standard aqueous solutions or in diluted wines.

$S$-CPEs prepared from different varieties of grape showed characteristic electrochemical responses, indicating that each skin grape has a specific phenolic composition which involves anthocyanin content and extractability (Rio Segade, Rolle, Gerbi, \& Orriols, 2008). Moreover, because the skin is a part of the sensitive layer, the electrochemical responses could also be influenced by structural and mechanical (porosity, hardness, thickness, etc.) properties of the skin. Al these factors produced distinctive responses and justify why these $S$ CPE sensors can be used to discriminate grapes of different varieties or grapes with distinct phenolic content in its skin.

Next objective was to evaluate the capability of the $S$-CPE sensors to monitor the changes taking place during ripening. For this purpose, sensors were prepared using the skin of grapes collected during five consecutive weeks starting from véraison.

Fluctuations in the berry compositions occurring during ripening 
Paper III: A different approach for the analysis of grapes: Using the skin as sensing element.

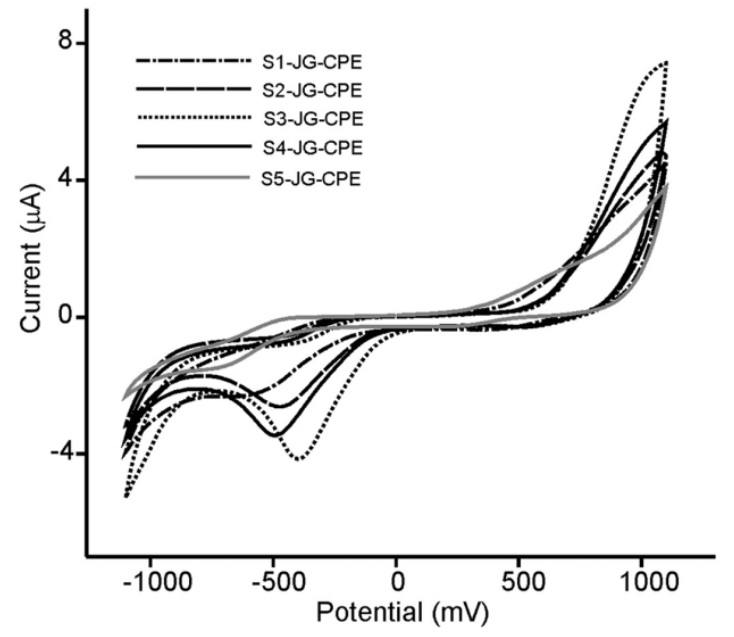

Fig. 2. Electrochemical response of $J G$-CPE electrodes along maturation. Cyclic voltammograms registered in $0.01 \mathrm{M}$ phosphate buffer $\mathrm{pH} 7.0$, Scan rate $0.1 \mathrm{~V} / \mathrm{s}$.

Table 3

Voltammetric parameters for the three skin grape-CPEs sensors: Mencía, Prieto Picudo and Juan García obtained in each sampling time.

\begin{tabular}{|l|l|l|l|l|l|l|}
\hline & \multicolumn{2}{|l|}{ Mencia-CPE } & \multicolumn{2}{l|}{ Prieto Picudo-CPE } & \multicolumn{2}{l|}{ Juan Garcia-CPE } \\
\hline $\begin{array}{l}\text { Anodic } \\
\text { peak }\end{array}$ & E (mV) & Imax $(\mu \mathrm{A})$ & E (mV) & Imax $(\mu \mathrm{A})$ & E (mV) & Imax $(\mu \mathrm{A})$ \\
\hline S1-S-CPE & 455 & 0.73 & 475 & 1.12 & 851 & 2.7 \\
\hline S2-S-CPE & 467 & 0.72 & 885 & 4.43 & 938 & 3.66 \\
\hline S3-S-CPE & 462 & 0.88 & 926 & 5.59 & 1030 & 7.09 \\
\hline S4-S-CPE & 744 & 1.89 & 916 & 5.50 & 1008 & 5.05 \\
\hline S5-S-CPE & 698 & 1.47 & 685 & 2.19 & 590 & 1.1 \\
\hline $\begin{array}{l}\text { Cathodic } \\
\text { peak }\end{array}$ & E (mV) & Imax $(\mu \mathrm{A})$ & E (mV) & Imax $(\mu \mathrm{A})$ & E (mV) & Imax $(\mu \mathrm{A})$ \\
\hline S1-S-CPE & -706 & -1.73 & -648 & -1.87 & -581 & -2.24 \\
\hline S2-S-CPE & -731 & -2.36 & -468 & -2.94 & -468 & -2.61 \\
\hline S3-S-CPE & -693 & -1.24 & -453 & -3.15 & -395 & -4.14 \\
\hline S4-S-CPE & -726 & -2.13 & -465 & -3.14 & -493 & -3.45 \\
\hline S5-S-CPE & -699 & -1.27 & -685 & -2.19 & -694 & 1.43 \\
\hline
\end{tabular}

caused important changes in the position and intensities of the peaks (Fig. 2). The main changes corresponded to the intensity of the peaks associated to phenols that increased in intensity from véraison until a maximum was attained. Then values decreased slightly near harvesting time. This result is in good agreement with variations measured using classical chemical techniques that establish that the level of anthocyanes increases during grape ripening and decreases slightly in ripened grapes (Cadot, Chevalier, \& Barbeau, 2011; Conde et al., 2007; Liang et al., 2011; Smith, 2011). The shift of the anodic wave towards higher potentials might be a demonstration of a higher polymerization level of the skin polyphenols. This may be corroborated by the shift of the cathodic wave to less negative potentials.

The extension of the changes detected depended on the variety of grape. The voltammetric parameters obtained from the three varieties of grapes, along maturation, are summarized in Table 3 . The maximum

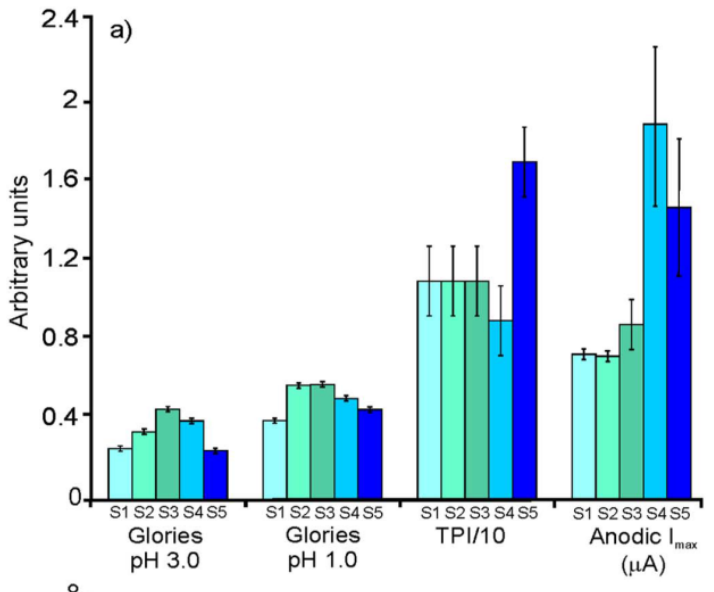

b)
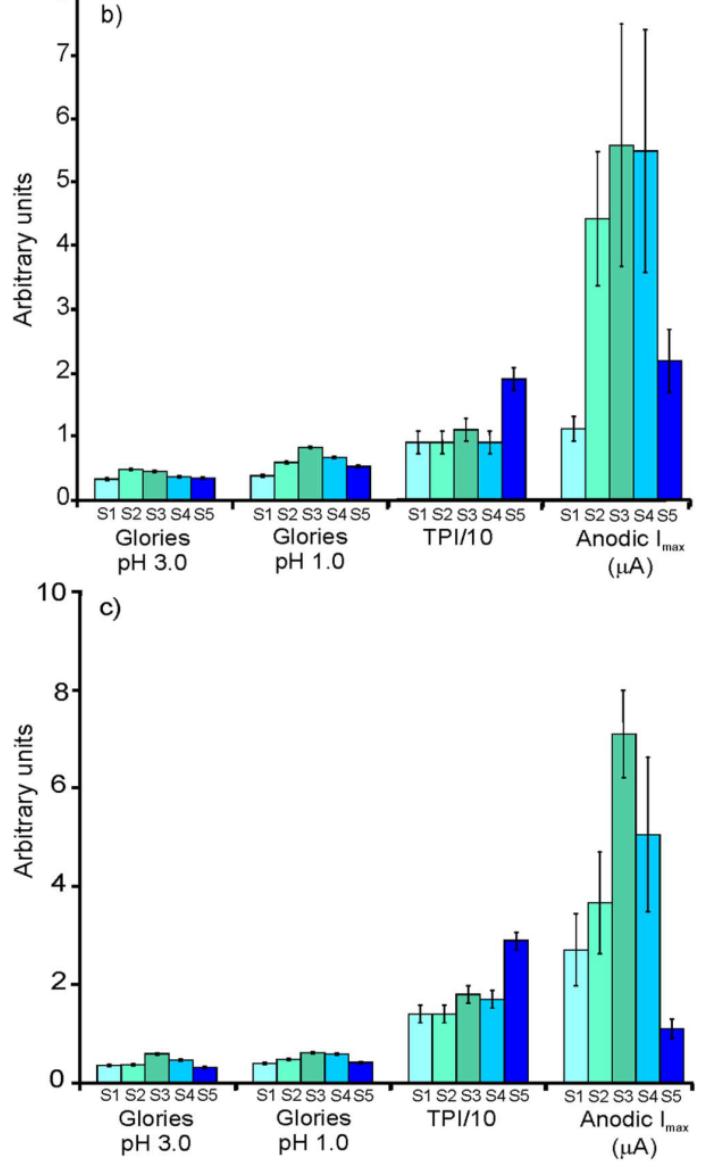

Fig. 3. Representation of the results obtained using Glories at extracts $\mathrm{pH} 3.0$ and $\mathrm{pH} 1.0$, IPT and intensity of the anodic peak for (a) M-CPE; (b) PP-CPE and (c) JG-CPE. 

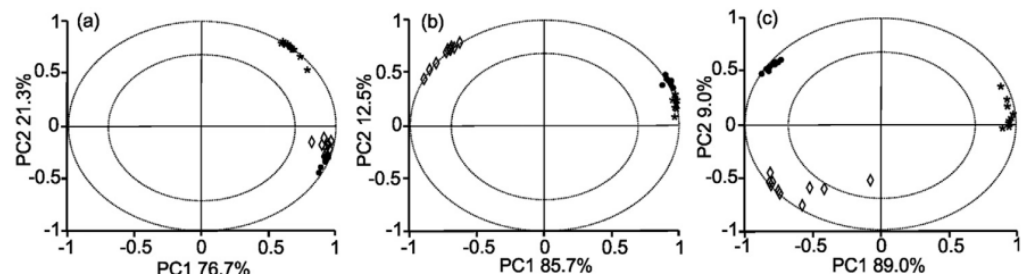

Fig. 4. Loading plot of the PCA. CPE-Mencía (•), CPEPrieto Picudo $(\Theta)$ and CPE-Juan García $(*)$ (ten kernels per sensor were extracted) represented at different maturation states (a) S1; (b) S3 and (c) S5.

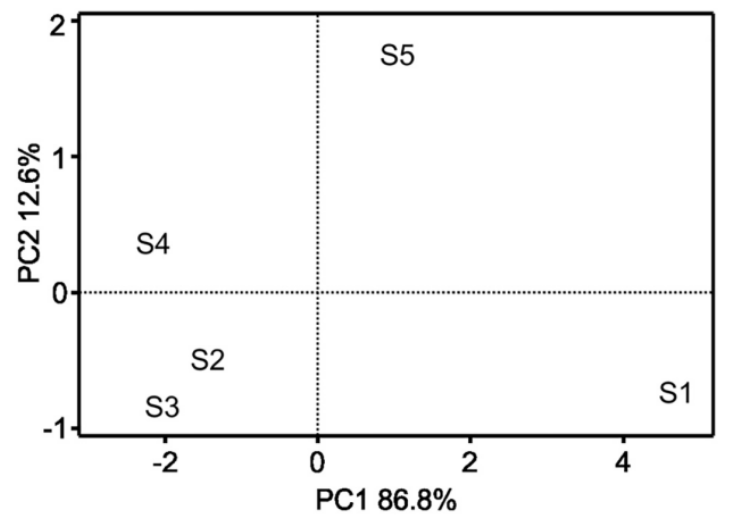

Fig. 5. Partial Least Squares Discriminant Analysis (PLS-DA) of JG-CPEs analyzed along maturation.

Table 4

Results of the PLS-1 analysis for $J G$-CPE

\begin{tabular}{llllll}
\hline Parameter & $\mathrm{Rc}^{\mathrm{a}}$ & $\mathrm{RMSEC}^{\mathrm{b}}$ & $\mathrm{Rp}^{\mathrm{c}}$ & $\mathrm{RMESP}^{\mathrm{d}}$ & $\mathrm{LV}^{\mathrm{e}}$ \\
\hline Glories pH 3 & 0.7204 & 0.0516 & 0.6718 & 0.0572 & 3 \\
Glories pH 1 & 0.7858 & 0.0397 & 0.7511 & 0.0437 & 3 \\
TPI & 0.8376 & 2.2304 & 0.8017 & 2.5207 & 3 \\
Brix & 0.6967 & 0.9414 & 0.6262 & 1.0687 & 3 \\
Density (g/mL) & 0.6913 & 0.0041 & 0.6200 & 0.0047 & 3 \\
Sugars (g/L) & 0.6920 & 10.5732 & 0.6209 & 11.9976 & 3 \\
Degree (16.8) & 0.6918 & 0.6287 & 0.6205 & 0.7135 & 3 \\
Degree (17.5) & 0.6927 & 0.6023 & 0.6224 & 0.6828 & 3 \\
\hline
\end{tabular}

${ }^{\text {a }}$ Squared correlation coefficient in calibration.

${ }^{\mathrm{b}}$ Root Mean Square Error of Calibration.

${ }^{\mathrm{c}}$ Squared correlation coefficient in prediction.

${ }^{\mathrm{d}}$ Root Mean Square Error of Prediction.

${ }^{\mathrm{e}}$ Latent variables.

Table 5

Results of the PLS-1 analysis for PP-CPE.

\begin{tabular}{llllll}
\hline Parameter & $\mathrm{Rc}^{\mathrm{a}}$ & $\mathrm{RMSEC}^{\mathrm{b}}$ & $\mathrm{Rp}^{\mathrm{c}}$ & $\mathrm{RMESP}^{\mathrm{d}}$ & $\mathrm{LV}^{\mathrm{e}}$ \\
\hline Glories pH 3 & 0.6177 & 0.0371 & 0.5616 & 0.0406 & 3 \\
Glories pH 1 & 0.8464 & 0.0581 & 0.8175 & 0.0648 & 3 \\
TPI & 0.6740 & 2.2142 & 0.5606 & 2.6292 & 4 \\
Brix & 0.6598 & 0.7149 & 0.5632 & 0.8284 & 5 \\
Density (g/mL) & 0.6410 & 0.0033 & 0.4875 & 0.0040 & 4 \\
Sugars (g/L) & 0.6419 & 8.3456 & 0.4888 & 10.1972 & 4 \\
Degree (16.8) & 0.6416 & 0.4950 & 0.4884 & 0.6049 & 4 \\
Degree (17.5) & 0.6431 & 0.4792 & 0.4903 & 0.5857 & 4 \\
\hline
\end{tabular}

${ }^{a}$ Squared correlation coefficient in calibration.

${ }^{\mathrm{b}}$ Root Mean Square Error of Calibration.

${ }^{\mathrm{c}}$ Squared correlation coefficient in prediction

${ }^{\mathrm{d}}$ Root Mean Square Error of Prediction.

${ }^{\mathrm{e}}$ Latent variables. intensity is indicated in the table using a grey background.

The peak potentials and intensities of the anodic and cathodic waves of the three varieties of $S$-CPE sensors increased with time and decreased in the last sampling corresponding to the official date of harvest, decided by oenologists, using the Brix degree and the sugar content.

Comparing the three varieties of grapes, Mencía showed the slower polyphenolic skin maturation and the maximum redox intensity was observed in the S4 sampling. The skins of Prieto Picudo and Juan García grapes reached the maximum redox intensity in the S3 sampling, indicating a faster ripening. As shown in Fig. 3, the intensity of the redox peaks obtained from the three types of skin-based sensors along maturation, followed a similar trend than the absorbance value of grape extracts at $280 \mathrm{~nm}$ at $\mathrm{pH} 3.0$ and $\mathrm{pH} 1.0$ obtained with the Glories method, in which polyphenols are extracted from the whole grape. The advantage of the $S$-CPE sensors is that variations are more marked than in Glories and the decrease previous to maturation is easier to detect This fact was specially observed in JG-CPE, corresponding to Juan García skin grapes, that seems to show faster polyphenolic skin maturation and a higher amount of extractable polyphenols. The variations of the Total Polyphenol Index (TPI) follow a different trend and tend to increase from véraison to complete maturity. So, TPI does not show an inflexion point that could be used to establish the optimal harvesting date. According to these results, the use of the skin of grapes as the sensitive layer provides an easy and direct method to assess the phenolic maturity of grapes.

Inspired in holistic analytical methods where arrays of sensors are combined with a pattern recognition software (Merkyte, Morozova, Boselli, \& Scampicchio, 2017; Pigani et al., 2018; Rodriguez-Méndez, de Saja, Medina-Plaza, \& Garcia-Hernandez, 2016; Sliwinska, Wisniewska, Dymerski, Namiesnik, \& Wardenci, 2014), the electroanalytical capacity of the $S$-CPE electrodes to monitor grape maturation was evaluated using Partial Least Squares-Discriminant Analysis (PLS-DA) and Principal Component Analysis (PCA).

PCA loading plot showed that $M$-CPE, PP-CPE and $J G$-CPE prepared in $\mathrm{S} 1$ (just one week after véraison), appeared in similar areas of the diagram (Fig. 4). Ripening caused progressive changes in the molecular and mechanical properties of skins and in their porosity. In the last sampling corresponding to mature grapes, the signals of $M$-CPE, PP-CPE and $J G$-CPE skin-based sensors appeared in different regions of the diagram, confirming that each grape possess a characteristic phenolic composition and the three varieties of grapes could be clearly discriminated.

Fig. 5 shows the Partial Least Squares-Discriminant Analysis (PLSDA) for PP-CPE samples collected along the maturation (five samplings). It was observed that the first Principal Component shifted to negative values as the ripening advanced. Then, five weeks after véraison, the PC1 returned to positive values. This circular behavior has already been observed using multisensors systems based on electrochemical electrodes (Medina-Plaza et al., 2016). PCA results found in Mencía and Prieto Picudo were similar and the clusters corresponding to the progressive maturation of grapes turned clockwise and tended to return to the initial position (data not shown)

A PLS-1 multiparametric model was established to find correlations between the results obtained with our skin sensors and the chemical 
Paper III: A different approach for the analysis of grapes: Using the skin as sensing element.

Table 6

Results of the PLS-1 analysis for M-CPE.

\begin{tabular}{|c|c|c|c|c|c|}
\hline Parameter & $\mathrm{Rc}^{\mathrm{a}}$ & RMSEC $^{b}$ & $\mathrm{Rp}^{e}$ & RMESP $^{\text {A }}$ & $\mathrm{IV}^{\mathrm{e}}$ \\
\hline Glories $\mathrm{pH} 3$ & 0.5129 & 0.0561 & 0.2143 & 0.0728 & 10 \\
\hline Glories pH 1 & 0.3603 & 0.0563 & 0.0336 & 0.0708 & 10 \\
\hline $\mathrm{TPI}$ & 0.6916 & 1.5065 & 0.5394 & 1.8829 & 5 \\
\hline 'Brix & 0.3472 & 0.7962 & -0.1142 & 1.0638 & 10 \\
\hline Density $(\mathrm{g} / \mathrm{mL})$ & 0.3379 & 0.0035 & -0.1231 & 0.0047 & 10 \\
\hline Sugars $(\mathrm{g} / \mathrm{L})$ & 0.3386 & 8.9652 & -0.1239 & 11.9524 & 10 \\
\hline Degree (16.8) & 0.3401 & 0.5305 & -0.1198 & 0.7067 & 10 \\
\hline Degree (17.5) & 0.3379 & 0.5012 & -0.1257 & 0.6684 & 10 \\
\hline
\end{tabular}

a Squared correlation coefficient in calibration.

Root Mean Square Error of Calibration.

Squared correlation coefficient in prediction.

Root Mean Square Error of Prediction

Latent variables.

parameters obtained by classical chemical techniques. Voltammograms can bring information not only about the polyphenolic content, but also about other components present in the skin (sugars, acids or ions) that can affect the electrochemical response. For this reason, attempts were made to use the $S$-CPEs to assess not only the phenolic content, but also to determine simultaneously other indicators of maturity.

Tables 4, 5 and 6 show the statistical parameters for the PLS-1 regression for calibration and validation (cross-validation). The root mean square errors and the correlation coefficients at calibration (RMSEC) and prediction (RMSEP) are shown in the tables. Rc (and Rp) are correlation coefficients of calibration (and prediction). LV indicates the number of latent variables used in the calculations.

Good relationships were found between the responses provided by the JG-CPE (Table 4) and PP-CPE sensors (Table 5), and the phenolic content measured by Glories and TPI. Higher correlation coefficients were found with Glories at $\mathrm{pH} 1.0$ than at $\mathrm{pH} 3.0$. The extracts at $\mathrm{pH} 1.0$ take account of the total extractable polyphenols, whereas at $\mathrm{pH} 3.0$ only easily extractable polyphenols are measured. Phenolic extractability depends of their cellular localization and degree of polymerization (Gagné, Saucier, \& Gény, 2006). These data indicate that JGCPE and PP-CPE sensors can detect most of the polyphenolic content of the skin. In the case of TPI, correlations coefficients were not so high because this method has interferences from non-phenolic compounds that contain aromatic rings (nucleotides, aromatic amino acids, peptides or proteins). Moreover, because the whole voltammograms was used to build the mathematical model, electrochemical data bring information not only about phenols but also about other components of the skin. For this reason, good correlations were also found with other parameters of interest in oenology including sugar, degree brix, grade and density.

However, low correlations were found using the M-CPE (Table 6). This lack of correlation might be attributed to the delay in the phenolic maturation process of Mencia grapes and to the different properties of their skins. In addition, it is known that the porosity and the structural properties, such as number of cell layers from cuticle to flesh, and mechanical properties of the skins, such as hardness, vary from one variety of grape to another and are also influenced by environmental factors (Rolle, Gerbi, Schneider, Spanna, \& Río Segade, 2011). The evolution of skin hardness during the ripening period is not completely known, although it considered an adequate parameter for the estimation of the extractability of phenol compounds (Rolle et al., 2011). An important observation in our study is the higher difficulty to separate skin and flesh in Mencía grapes. In this sense, the degree of adhesion between skin and flesh, along ripening, was more variable in this variety that for Juan García and Prieto Picudo, varieties where the skin and flesh were properly separated in every sampling. For instance in sampling S3, Mencía grapes were collected after a heavy rain, and skin and flesh could hardly be separated. These facts might have interfered in the permeability of the skin and in consequence with the diffusion of ions and electron transfer from the skin to the electrode.

\section{Conclusions}

Skin-CPE electrodes fabricated by covering a carbon electrode with the skin of grapes can be used to evaluate the phenolic content of grapes. The redox processes at electrode surface, provided information about phenolic compounds present in the skin. The electrochemical response of the three different skin-sensors electrodes along the maturation, could be used to monitor the ripening process. Good correlations were found with other parameters suh as sugar content or ${ }^{\circ} \mathrm{Brix}$ usually used to establish the optimal maturity. The phenolic maturation process of the vintage and the structural and mechanical skin grape properties, are esential factors, to found good correlations between the responses provided by $S$-CPE sensors and chemical data measured by classical chemical methods. Thus the skin sensors fabricated with Juan Garcia and Prieto Picudo grapes, that showed a faster polyphenolic skin maturation and a higher amount of extractable polyphenols than Mencía variety, showed a very good correlations and therefore could be used to monitor the ripening of Juan García and Prieto Picudo grapes. Althought the presented work brings a new and original approach to monitor the phenolic maturity, it might be noticed that the elaboration of the sensors must be carried out very carefully when peeling off the grape skin.

\section{Acknowledgements}

Financial support from CICYT-FEDER (Grant $n^{\circ}$ AGL2015-67482-R) and Junta de Castilla y León (Ref. VA011U16) is gratefully acknowl edged. CGH thanks for the grant of JCYL (D-24112015-9).

In memoriam of José Antonio de Saja.

\section{Conflict of interest}

The authors report no conflict of interest.

\section{References}

Adams, D. O. (2006). Phenolics and ripening in grape berries. Amertican Joumal of Enology and Viticulture, $57,249-256$

Apetrel, C., Apetrel, 1. M., de Saja, J. A., \& Rodriguez-Méndez, M. L. (2011). Carbon paste electrodes made from different carbonaceous materials: Application in the study of antioxidants, Sensars, 11, 1328-1344.

Barroso, M. F., Santos-Alvarez, N., Delerue-Matos, C., \& Olivelm, M. B. P. P. (2011). Towards a reliable technology for antioxidant capacity and oxidative damage eva uation: Electrochemical (blo)sensors. Blasensors \& Bioelectrontes, 30, 1-12. Blasco, A. J., González-Crevillén, A. G., González, M. C., \& Escarpa, A. (2007). Direct electrochemical sensing and detection of natural antioxidants and antioxidant $\mathrm{ca}$. pacity in vitro systems. Electroanatysis, $19,2275-2286$.

Bordonaba, J. G., \& Terry, L. A. (2012). Electrochemical behaviour of polyphenol rich fruit juices using disposable screen-printed carbon electrodes: Towards a rapid senso for antioxidant capacity and individual antioxidants. Talanta, 90, 38-45.

Buratti, S., Scampicchio, M., Giovanelli, G., \& Mannino, S. (2008). A low-cost and lowtech electrochemical flow system for the evaluation of total phenolic content and antioxidant power of tea infusions. Talanta, 75, 312-316.

Cadot, Y., Chevalier, M. \& Barbeau, G. (2011). Evolution of the localization and com. position of phenolics in grape skin between veraison and maturity in relation to wate availability and some climatic conditions. Journal of the Science of Food an griculure, 91, 1963-1977.

Conde, C., Silva, P., Fontes, N., Dias, A. C. P., Tavares, R. M., Sousa, M. J., ... Gerós, H. 2007). Biochemical changes throughout grape berry development and fruit and wine quallty. Food, 1, 1-22

Durst, R. A., Baumner, A. J., Murray, R. W., Buck, R. P., \& Andrieux, C. P. (1997). Chemically modified electrodes: Recommended teminology and definitions. Pure and Appted Chemistry, 69, 1317-1323.

(2006). Composition and cellular localization of tarnins Cabernet Sauvignon skins during growth. Journal of Agrtcultural and Food

García-Hernández, C., García-Cabezón, C., Martin-Pedrosa, F., de Saja, J. A., \& Rodríguez. Mendez, M. L. (2016). Layered composites of PEDOT:PSS/nanoparticles and . Belstein

Journal of Nanotechnology, 7, 1948-1959.
Garrido, J., \& Borges, F. (2013). Wine and grape pholyphenols-A chemical perspective. 
Chapter 3: Results and discussion

Paper III: A different approach for the analysis of grapes: Using the skin as sensing element.

Glories, Y., \& Augustin, M. (1993). Maturité phénolique du raisin, consequences technologiques: Application aux millés'mes 1991 et 1992. Journée Tecnique du C.LV.B. Actes du

Gonzallez.San José, M. L., Barren, L. J. R., \& Diez, C. (1990). Evolution of anthocyanins during maturation of Tempranillo grape cultivar (Vitis vinifera) using polynomial regression models. Journal of the Science of Food and Agriculture, 51, 337-344. Application of principal component analysis to ripening indices for wine grapes.
(n) Application of principal component analysis to ripening
Journal of Food Compositton and Analysis, 4, 245-255.

Hoyos-Arbelaez, J., Vazquez, M., \& Contreras-Calderon, J. (2017). Electrochemical methods as a tool for determining the antloxidant capacity of food and beverages: A
mas. methods as a tool for determining the antoxi
review. Food Chemistry, 221, 1371-1381.

Jackson, R. S. (2014). Wine science: Principles and applications. Amsterlam, The Netherlands: Elsevier Academic Press.
Nes

Jara-Polacios, M. J., Hernanz, D. Escudero-Gilete, M. L. \& Heredia, F. (2014) Antioxidant potential of white grape pomaces: Phenolic composition and antioxidant capacity measured by spectrophotometric and cyclic voltammetry methods. Food Research intermational, 66, 150-157.

Kennedy, J. A. (2008). Grape and wine phenolics: Observations and recent findings.

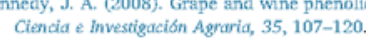

Kilmartin, P. A. (2013). Electrochemistry applied to the analysis of wine: A mini review. Electrochemistry Communications, $67,39-42$.

Kirsanov, D. Mednova, O., Vietoris, V., KIlmartin, P. A., \& Legin, A. (2012). Towards reliable estimation of an "electronic tongue" predictive ability from PI.S regression models in wine analysis. Talartan, $90,109-116$.

Liang, Z., Sang, M. Fan, P. Wu, B., Wang, I, Duan, W., \& Li, S. (2011) Changes of polyphenols, sugars, and organic acid in 5 Vitis genotypes during berry ripening polyphenols, sugars, and organic acid in

Iohitnavy, N., Bastian, S. \& Collins, C. (2010). Berry sensory attributes correlate with compositional changes under different viticultural management of Semillon (Vitis vinifera 1.). Food Quality and Preference, 21, 711-719.

Mahmood, T., Anwar, F., Abbas, M., \& Saart, N. (2012). Effect of maturity on phenolics (phenolic acids and favonoids). Drofle of strawberry cultivars and mulbemy species from Pakistan. International Journal of Molectular Sciences, 13, 4591-4607.

Makhotkina, O., \& Kllmartin, P. A. (2010). The use of cyclic voltammetry for wine analygiss Determination of polyphenols and fres sulfur dioxide. Analitica Chimica Ath. $668,155-165$.

Makhotkina, O., \& Kilmartin, P. A. (2013). Electrochemical oxidation of wine polyphenols in the presence of sulfur dioxide. Journal of Agricultural and Food Chemistry, 61. 5573-5581.

Medina-Plaza, C., de Saja, J. A., Fernández-Escudero, J. A, Barajas, E., Medrano, G., \& Rodriguez-Méndez, M. L. (2016). Array of biosensors for discrimination of grapes
according to grape variety, vintage and ripeness. Analytica Chirnica Acta, 947, 16-22.

Medina-Plaza, C., Gareia-Hernandez, C., de Saja, J., Fernández-Escudero, J. A., Barajas, E, Medrano, G.,... Rodriguez-Méndez, M. L. (2015). The advantages of disposable
screen-printed biosensors in a bloelectronic tongue for the analysis of grapes. LWT. Food Science and Techrology, 62, 940-947.

Merkyte, V., Morozova, K., Boselli, E, \& Scampicchio, M. (2017), Fast and simultaneous determination of antioxidant activity, total phenols and bitterness of red wines by a
multichannel amperometric electronic tongue. Electrodratysis. Wiley Online Library.

Negri, A. S., Prinsi, B., Rossoni, M., Failla, O., Scienza, A., Cocucci, M., \& Espen, L. (2008). Proteome changes in the skin of the grape cultivar Barbera among different stages of ripening. BMC Genomics, 9, 1-19.

Nogales-Bueno, J., Baca-Bocanegra, B., Rooney, A, Hernández-Hierro, J. M., Byme, H. J., \& Heredia, F. J. (2017), Study of phenolic extractability in grape seeds by means of
ATR-FTIR and Raman spectroscopy. Food Chemistry, 232, 602-609.

OIV (2013). Compendium of theernational methods of aralysis of wines and musts. VoL. 2 (Paris)

Piganl, L, Simone, G. V., Foca, G., Ulirici, A., Masino, F., Cubillana-Agullera, L., ... Seeber, R. (2018). Prediction of parameters related to grape ripening by multivariate cal oration of voltammetric signals accquired by an electronic tongue. Talanta, 178 178-187.

Piljac-Žegarac, J., Valek, L., Stipčevic, T., \& Martinez, S. (2010). Electrochemical determination of antioxidant capacity of fruit tea infusions. Food Chemistry, 12 820-825.

Pisoschl, A. M., Cheregl, M. C., \& Danet, A. F. (2009). Antioxidant capacity of some ommercial fruit juices: Electrochemical and spectrophotometrical approaches. Molecules, $14,480-493$.

Rio Segade, S., Rolle, 1., Gerbi, V., \& Orriols, I. (2008). Phenolic ripeness assessment of grape skin by texture analysis. Journal of Food Composition and Analysis, 21, 644-649. ren, L. J. (1991)

events during ripening of grape berries. Italian Joumal of Food Science, 3, $173-180$

Rockenbach, I. L., Valdemiro, I.., Rizelio, V. M. de Souza, A. E., Genovese, M. I. \& Fett, R. (2011). Phenolic compounds and antioxidant activity of seed and skin extracts of red grape (Vtets vitifera and Vitts labrusea) pomace from Brazllian winemaking. Food Reseach International, 44,897-901.

Rodriguez-Mendez, M. L. (2016). Electronte noses and tongtes th the food tidtustry. ElsevierAcademic Press.

Rodr Cheynier, V. (2014). Evaluation of oxygen exposure levels and polyphenolic concent of red wines using an electronic panel formed by an electronic nose and an electronic tongue. Food Chemistry, 155, 91-97.

Rodriguez-Mendez, M. L, de Saja, J. A., Medina-Plaza, C., \& Garcia-Hernandez, C. (2016) Electronic tongues for the organoleptic characterization of wines. Electronic noses ard Eectronic tongues for the organoleptic characterzation of wines. Electronte noses ard
congues in the food industry (pp. 265-273). Amsterdam, The Netherlands: Elsevier Academic Press.

Rolle, L, Gerbi, V., Schneider, A., Spanna, F., \& Rio Segade, S. (2011). Varietal relationship between instrumental skin hardness and climate for grapevines (Vitis vi. niffera L.). Joumal of Agrtcultural and Food Chemistry, 59, 10624-10634.

Rudnitskaya, A., Schnidtke, L. M., Reis, A., Domingues, M. R., Delgadillo, I., Debus, B., Legin, A. (2017). Measurements of the effects of wine maceration with oak chips using an electronic tongue. Food Chemistry, 229, 20-27.

Sliwinska, M., Wisniewska, P., Dymerski, T., Namiesnik, J., \& Wardenct, W. (2014). Food analysis using artificial senses. Journal of Agricultural and Food Chemistry, 62, $1423-1448$.

Smith, C. (2011). Phenol chemistry and winemaking. Wines and Vines, 54-59 (April). Sokolowsky, M., Rosenberger, A., \& Fischer, U. (2015). Sensory impact of skin contact on white wines characterized by descriptive analysis, time-intensity analysis and temphite wines characterized by descriptive analysis, time-intensity analysis and tern-

Yang, C., Denno, M. E., Pyakurel, P., \& Venton, B. J. (2015). Recent trends in carbon nanomaterial-based electrochemical sensors for biomolecules: A review. Analitica Chimica Acta, 887, 17-37.

Zielinska, D., Wiczkowski, W., \& Piskula, M. K. (2008). Determination of the relative contribution of quercetin and its glucosides to the antioxidant capacity of onion by
cyclic voltammetry and spectrophotometric methods. Joumal of Agricultural and Food Chemistry, 56, 3524-3531.

Ziyatdinova, G., Kozlova, E., \& Budnikov, H. (2016). Chronocoulometry of wine on multiwalled carbon nanotube modified electrode: Antioxidant capacity assay. Food Chemistry, 196, 405-410. 



\subsection{Paper IV}

Electrochemical behavior of polypyrrol/AuNP composites deposited by different electrochemical methods: sensing properties towards catechol.

By Celia García-Hernández, Cristina García-Cabezón, Cristina MedinaPlaza, Fernando Martín-Pedrosa, Yolanda Blanco, José Antonio de Saja and María Luz Rodríguez-Méndez.

Beilstein Journal of Nanotechnology 6 (2015) 2052-2061.

(doi: 10.3762/bjnano.6.209) 


\subsubsection{Motivation and objective}

Organic $\pi$-conjugated polymers have received much attention for sensing applications. Some of the most used CPs for industrial applications or fundamental research have been polyacetylene (PA), polyaniline (PANI), polypyrrole (Ppy) and polythiophene (PT). In general, CPS have attractive properties, such as electrical conductivity, workability, low cost, light weight, resistance to corrosion, high electron affinity, redox activity and excellent mechanical and optical properties.

In a neutral state, conjugated polymers behave as insulators; however, their conductivity can be increased by doping through the oxidation (p-doping) and reduction (n-doping) using chemical or electrochemical methods. For example, the backbone of Ppy is neutral in the reduced state and positive in the oxidized state, needing a counterion that diffuses into the polymer during charging and out during neutralization, maintaining electroneutrality. When the conducting polymers are exposed to higher oxidative potentials than their standard, an overoxidation is produced, causing a de-doping and therefore loss of electrical conductivity.

Ppy films have been prepared by means of electrochemical reactions more than by chemical synthesis. However, other polymerization methods that have been reported for Ppy preparation include photochemistry, solid-state, plasma, pyrolysis, metathesis and/or concentrated emulsion. However, the electrochemical methods are the most suitable to prepare thin solid films due to various factors: the simplicity of the technique, easy control of film thickness, facility of doping during the synthesis, the availability of several dopant ions and the formation of films with a good quality. In this way, polypyrrole is electrodeposited onto a positively polarized working electrode by a condensation reaction from the bulk solution phase of monomer units, which also contains a negatively charged counterion that maintains the electroneutrality along the Ppy backbone that will have positive charges.

Conducting polymers have been widely used to prepare composites materials, along with other sensitive materials with electrochemical properties, such as metal nanoparticles, carbon materials, metal oxide nanoparticles, MOFs, dyes and/or enzymes to be implemented in several applications, including electrochemical sensors. For example, composites of CPs and carbonbased materials can improve selectivity for target molecules in sensor devices. Moreover, the combination of metal and metal nanoparticles with conducting polymers for electrochemical 
device purposes can increase the electron transfer between the electrolyte and the electrode. Metal and metal oxide nanoparticles exhibit a high performance with respect to their bulk state as electrode materials. For instance, AuNPs have unique electrochemical properties for sensing applications and can be synthesized by means of several methods, including wet chemical synthesis, electrochemical deposition or monolayer formation. Therefore, conducting polymer/AuNPs composites are good candidates to modify electrode surfaces and improve the electrochemical performance of sensors.

The objective of this work is to develop new Ppy/AuNPs composites and study the factors that can influence their conductivity and electrochemical behavior, such as the electrodeposition technique used to prepare Ppy films (chronoamperometry or chronopotentiometry), the way of incorporating AuNPs into the polymeric matrix, the importance of the selected substrate (platinum or stainless steel), as well as the influence of the electrolyte solution in sensor performance. The electrochemical responses of the as-prepared sensors were studied for catechol and the selected sensors with the best performances will be further implemented in another experimental work to analyze grapes and wines.

\subsubsection{Summary of the research work}

The electrochemical sensors were prepared by electrodepositing Ppy films from an aqueous solution containing pyrrole and sodium 1-decanesulfonate (DSA), which was used as the counterion to maintain the electroneutrality of the Ppy films. Two electrochemical techniques were tested: chronopotentiometry (CP) at a constant potential of $0.8 \mathrm{~V}$ for $300 \mathrm{~s}$, and chronoamperometry (CA) at a constant current of $0.02 \mathrm{~mA}$ for $300 \mathrm{~s}$. Ppy/AuNPs were prepared following two strategies: in the first, referred to as the trapping method, AuNPs were previously synthesized using the well-known procedure proposed by Slot and Geuze and then mixed with the solution of pyrrole and DSA before the electrodeposition. In the second, referred to as the cogeneration method, a solution of tetrachloroauric acid $\left(\mathrm{HAuCl}_{4}\right)$ was mixed with the solution containing pyrrole and DSA and further electropolymerization was carried out to form in situ AuNPs simultaneously with the deposition of the film. Moreover, Ppy and Ppy/AuNPs films were deposited onto platinum and stainless steel substrates. 
Electropolymerization experiments, both chronopotentiomtry and chronoamperometry, evidenced that the presence of AuNPs or tetracloroauric acid in the solution of pyrrole and DSA impeded the oxidation of the monomers, making the final oxidation potential stay over the potential at which the monomer is oxidized. AuNPs previously synthesized impeded the oxidation of the monomer even more than the AuNPs generated in situ. The structural characterization by SEM confirmed the incorporation of AuNPs with an average size of 30-40 nm, uniformly dispersed in the Ppy matrix. The structures of films deposited onto platinum (Pt) and stainless steel (SS) were almost identical. However, the number of AuNPs incorporated into the Ppy matrix was higher when using $\mathrm{CP}$ and the cogeneration method.

Electrochemical impedance spectroscopy was carried out in order to study the conductivity of the films. In platinum substrate, AuNPs inserted into a Ppy matrix evidenced a change in the electrical behavior of the sensors, reducing the resistance by facilitating the electron transfer. Moreover, the conductivity of films improved in those prepared by chronopotentiometry and inserting AuNPs by the cogeneration method, which turned out to be the most suitable for inserting a higher amount of AuNPs. In stainless steel substrate, EIS experiments of films deposited by CA were irreproducible, indicating that films were unstable. Results obtained by CP produced reproducible responses, but the electron-transfer resistance and impedance values were higher for SS than for those obtained in platinum substrate, corroborating the fact the conductivity of films on Pt substrate was higher.

The electrochemical behavior of Ppy and Ppy/AuNP sensors was studied by cyclic voltammetry in a $\mathrm{KCl}$ electrolyte solution. The first scan was always different from the subsequent cycles. Ppy sensors showed two redox processes in the first scan, corresponding to the polaron and bipolaron of Ppy. In the next cycles, a broad oxidation peak and a cathodic peak were observed. Results were similar for both CA or CP; however, the substrate played an important role, the intensities obtained when using SS substrate were lower than in the case of using Pt.

The electrocatalytic performance of AuNPs was also confirmed by cyclic voltammtrey. In platinum substrate, Ppy/AuNP films generated by CP and CA showed an important increase in the intensities compared to Ppy films. Moreover, the separation between the anodic and cathodic peaks was slightly reduced. In stainless steel substrates, a decrease in the intensities for Ppy/AuNP films compared to Ppy/AuNP films on Pt was observed. This result points to an interference factor between AuNPs and SS. Moreover, Ppy/AuNP films deposited on Pt substrate 
were more stable and reproducible over time than films obtained onto SS substrates. However, that irreproducibility was assigned to the electrolyte solution, specifically to a pitting process produced by chloride ions. Changing the electrolyte solution to phosphate buffer did improve the reproducibility of films deposited onto SS substrates.

The electrochemical sensing behavior of the Ppy and Ppy/AuNP films was also investigated for catechol. The electrochemical response was characterized by the two-electron redox process, due to the oxidation of catechol to benzoquinone. AuNPs increase the intensities of the peaks and also their reversibility. This effect was stronger when using CP instead of CA as the amount of AuNPs was higher using the chronopotentiometry method. The method of inserting AuNPs (trapping or cogeneration) did not strongly influence the results, probably due to the minimal differences in the concentration of the AuNPs. Limits of detection in catechol were in the range from $10^{-5}$ to $10^{-6} \mathrm{~mol} / \mathrm{L}$, lower than concentration values found in foods and beverages. AuNPs in Ppy films improved the limits of detection by almost one order of magnitude and, in general, the limits were lower when using Pt substrate, showing higher sensitivities and regression coefficients. 

Paper IV: Electrochemical behavior of polypyrrol/AuNP composites deposited by different electrochemical methods: sensing properties towards catechol.

\title{
Electrochemical behavior of polypyrrol/AuNP composites deposited by different electrochemical methods: sensing properties towards catechol
}

\author{
Celia García-Hernández ${ }^{1}$, Cristina Garcia-Cabezón ², Cristina Medina-Plaza ${ }^{1}$, \\ Fernando Martín-Pedrosa ${ }^{2}$, Yolanda Blanco ${ }^{2}$, José Antonio de Saja ${ }^{3}$ \\ and María Luz Rodríguez-Méndez ${ }^{* 1, \S}$
}

\section{Full Research Paper}

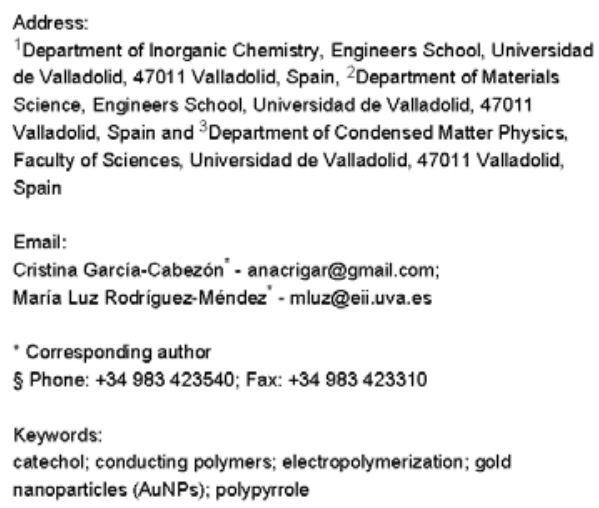

Abstract

Two different methods were used to obtain polypyrrole/AuNP (Ppy/AuNP) composites. One through the electrooxidation of the pyrrole monomer in the presence of colloidal gold nanoparticles, referred to as trapping method (T), and the second one by electrodeposition of both components from one solution containing the monomer and a gold salt, referred to as cogeneration method (C). In both cases, electrodeposition was carried out through galvanostatic and potentiostatic methods and using platinum (Pt) or stainless steel (SS) as substrates. Scanning electron microscopy (SEM) demonstrated that in all cases gold nanoparticles of similar size were uniformly dispersed in the Ppy matrix. The amount of AuNPs incorporated in the Ppy films was higher when electropolymerization was carried out by chronopotentiometry (CP). Besides, cogeneration method allowed for the incorporation of a higher number of AuNPs than trapping. Impedance experiments demonstrated that the insertion of AuNPs increased the conductivity. As an electrochemical sensor, the Ppy/AuNp deposited on platimum exhibited a strong electrocatalytic activity towards the oxidation of catechol. The effect was higher in films obtained by $\mathrm{CP}$ than in films obtained by chronoamperometry (CA). The influence of the method used to introduce the AuNPs (trapping or cogeneration) was not so important. The limits of detection (LOD) were in the range from $10^{-5}$ to $10^{-6} \mathrm{~mol} / \mathrm{L}$. LODs attained using films deposited on platinum were lower due to a synergy between AuNPs and platinum that facilitates the electron transfer, improving the electrocatalytic properties. Such synergistic effects are not so pronounced on stainless steel, but acceptable LOD are attained with lower price sensors. 


\section{Introduction}

Polypyrrole (Ppy) is one of the most extensively studied, conducting polymers due to its good electrical conductivity and redox properties $[1,2]$. Ppy films can be easily generated by electropolymerization and used as a strong adherent layer using different electrochemical techniques [3]. Electrodes that are chemically modified with Ppy have good electrocatalytic activity. For this reason, they have been widely used as chemical sensors for the detection of a variety of substances. The structure and sensing properties of the Ppy films are considerably influenced by the electrochemical method used for the polymerization (potentiostatic, galvanostatic or potentiodynamic), by the electrochemical conditions (such as voltage, intensity, or scan rate), and by other experimental conditions such as the nature and concentration of the doping agent or the nature of the substrate [4]. This versatility can be used to better control the development of electrochemical sensors with the appropriate selectivity, reproducibility and sensibility towards a particular application.

Recently, composite nanomaterials based on conducting polymers and metal nanoparticles (NPs) of different metals have been developed. Gold nanoparticles (AuNPs) have attracted considerable interest because of their unique optical, electronic and catalytic properties [5-8]. Conducting polymer-gold nanoparticle composites exhibit improved physical and chemical properties over their single-component counterparts and are the focus of intensive research [9-12]. In the case of sensors, it has been reported that the insertion of NPs into the sensing layer provides remarkable properties compared to conventional polymeric matrices. Several examples have been reported in the literature. For instance, electrochemically deposited Ppy/AuNP films have demonstrated a great potential to detect DNA [13] ammonia gas at room temperature [14], caffeine [15] or hydroxylamine [16] among others

Ppy/AuNP composites can be prepared by chemical and electrochemical polymerization. Electrochemical methods provide a better control of the structure and properties of the composite by controlling the electrochemical conditions during film generation [17]. The electrodeposition of the composite can be achieved using different strategies [18], mainly through the electrooxidation of the monomer in the presence of colloida gold nanoparticles and the corresponding doping agent [19] but also by electrodeposition of polymer and metal from two separate solutions $[20,21]$ or by electrodeposition of both components from one solution containing a monomer and a metal salt [17]. Finally, layers of electrodeposited polypyrrole and gold nanoparticle films can also been obtained from a single solution where PPy chains served as the reductant of tetrachloroauric acid [22]
Most of the works devoted to the electrosynthesis of Ppy/AuNPs films, are often limited to establish recipes to prepare the films and to tests their electrocatalytic or sensing properties. It could be expected that the electrocatalytic and the sensing properties of the Ppy/AuNPs films directly depend on the polymerization conditions. However, the influence of the polymerization conditions in the properties of Ppy/AuNPs electrodes has not been yet studied.

One of the fields where electrochemical sensors are having an important success is in the detection of phenolic compounds which are strong antioxidant reagents present in foods, with beneficial effects on human health [23]. As phenols are electroactive compounds, they can be detected by amperometric or voltammetric techniques using graphite or platinum electrodes [24-26]. In addition, electrodes chemically modified with a variety of sensing materials (e.g., phthalocyanines or conducting polymers) have been successfully used as voltammetric sensors for the detection of antioxidants [27]. It has also been demonstrated that the combined use of electrocatalytic materials such as phthalocyanines and nanoparticles, can induce synergistic effects that increase the sensitivity of the sensors [28]. Following this idea, Ppy/AuNPs composites could be good candidates as electrocatalytic materials for the detection of phenols.

The objective of this work was to develop new voltammetric sensors based on electrodeposited Ppy/AuNps for the detection of catechol (an antioxidant of interest in the food industry) and to evaluate the influence of the electrodeposition method in their performance. For this purpose Ppy/AuNp films doped with 1-decanesulfonic acid (DSA) were deposited using different methods. The first approach consisted on the electrodeposition of the Ppy/AuNPs films from a solution containing the monomer and tetrachloroauric acid (denoted as "cogeneration", C) The second approach consisted of the electrodeposition of the Ppy/AuNPs composited from a solution containing the monomer and gold nanoparticles previously formed (denoted as "trapping" method, T). In both methods, electrodeposition was carried out by chronoamperometry (CA) and by chronopotentiometry (CP). Particular attention was paid to the study of the influence of the substrate used for the electrodeposition that was carried out onto classical platinum electrodes and on stainless steel substrates. This aspect could play a crucial role not only in the structure, properties and performance of the sensor but also in the final price.

The structure and sensing properties of voltammetric sensor modified with Ppy/AuNPs films prepared under different conditions were evaluated and compared. 


\section{Results and Discussion}

PPy/AuNPs films were prepared using two different approaches referred as "trapping method" and "cogeneration method", which are described in the Experimental section. The electropolymerization of pyrrole was generated under potentiostatic and galvanostatic conditions on both platinum and stainless steel substrates, resulting in the formation of nanocomposites based on gold nanoparticles within the polypyrrole layer.

\section{Electropolymerization of Ppy/AuNPs}

Figure 1 shows the potential $(E)$ vs time $(t)$ curves registered during the electrodeposition PPy/AuNPs films using a galvanostatic process. The figure compares the results obtained by the trapping and the cogeneration methods. The $\mathrm{CP}$ registered for Ppy (in the absence of AuNPs) is also shown for comparison. As expected, as the current pulse was applied, a sharp decrease in the potential was observed. This was due to the charge of the double layer capacitance that produces a nucleation process at the electrode surface. Then, at the potential at which the monomer is oxidized, a stabilization and growth step was attained, which was characterized by a "plateau", where the potential varied only slightly.

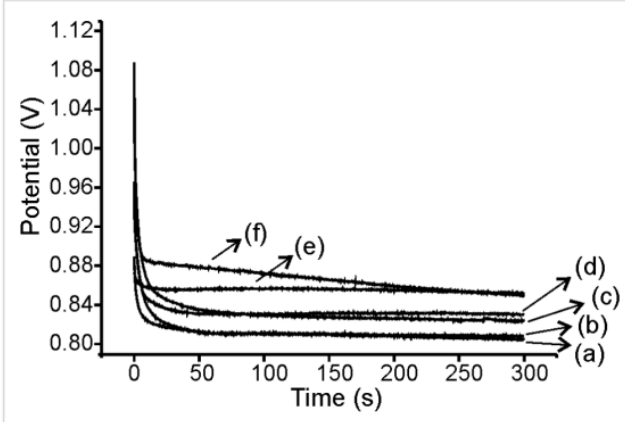

Figure 1: Chronopotentiometric curves obtained during the polymer-

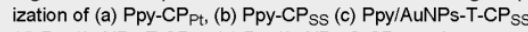
(d) Ppy/AunPs-T-CP $\mathrm{Pt}_{\mathrm{t}}$ (e) Ppy/AuNPs-C-CP $\mathrm{Pt}_{\mathrm{P}}$ and (f) Ppy/AuNPs-C-CPss.

The highest nucleation rate (faster electrode potential variation) was observed when Ppy was polymerized in the absence of gold nanoparticles or tetrachloroauric acid. At the same time, the final potential (at which the monomer is oxidized) was clearly lower for Ppy films. According to these results, it can be assumed that, the the presence of AuNPs affects the nucleation of Ppy, making impeding the oxidation of the monomers.

The final potential attained when polymerization was carried out in the presence of previously formed AuNPs (trapping), was lower than the potential obtained when AuNPs were generated in situ (cogeneration). This result seems to confirm that AuNPs affect the nucleation process. Only a small difference was found in the final potential attained by Ppy/AuNPs deposited on Pt or on SS.

Nanocomposites Ppy/AuNP were also prepared by trapping and cogeneration using CA. Curves show the characteristic stepped shape of the potentiostatic polymerization: After a short induction period where diffusion controls the monomer oxidation, the current increased rapidly with time, where polymer started nucleating and growing on the electrode surface. Finally, the current reached a plateau coinciding with a continuous and gradual polymer growth $[29,30]$. The calculated charges are shown in Table 1 .

Table 1: Polymerization charges calculated for Ppy and Ppy/AuNPs composites prepared by chronoamperometry.

\begin{tabular}{lcc} 
Sample & \multicolumn{2}{c}{$Q\left(\mathrm{C} / \mathrm{cm}^{2}\right)$} \\
& SS & Pt \\
\hline Ppy-CA & 0.62 & 0.62 \\
Ppy/AuNPs-T-CA & 0.07 & 0.08 \\
Ppy/AuNPs-C-CA & 0.12 & 0.22
\end{tabular}

In good accordance with results shown in previous paragraphs, also when using $\mathrm{CA}$, the polymerization charge was strongly dependent on the presence of AuNPs and the mass deposited in the absence of AuNPs was higher than the mass deposited in the presence of gold. The charge calculated for films obtained by cogeneration was higher than that of the films obtained by trapping. That is, the amount of polymer deposited followed the same trend regardless whether $\mathrm{CP}$ or CA was used (Ppy > Ppy/AuNP-C > Ppy/AuNP-T). This result also points to the role of AuNPs in the nucleation of Ppy, which impede the the oxidation of the monomers. The coefficients of variation (\% CV) were always lower than $2 \%$ regardless of the electropolymerization method or the susbstrate used.

\section{Structural characterization: SEM studies}

The microscopic structure of the Ppy/AuNP films analyzed by scanning electron microscopy confirmed the incorporation of the AuNPs into the Ppy films (Figure 2). They were uniformly dispersed in the typical granular raspberry PPy matrix. The structures of films deposited onto SS or Pt were almost identical.

The average size of the AuNPs was between 30 and $40 \mathrm{~nm}$ (regardless of the method used), which is consistent with the absorbance at $540 \mathrm{~nm}$ observed by colloid that was used to obtain the nanocomposites by trapping. The number of AuNPs 


\section{Chapter 3: Results and discussion}

Paper IV: Electrochemical behavior of polypyrrol/AuNP composites deposited by different electrochemical methods: sensing properties towards catechol.
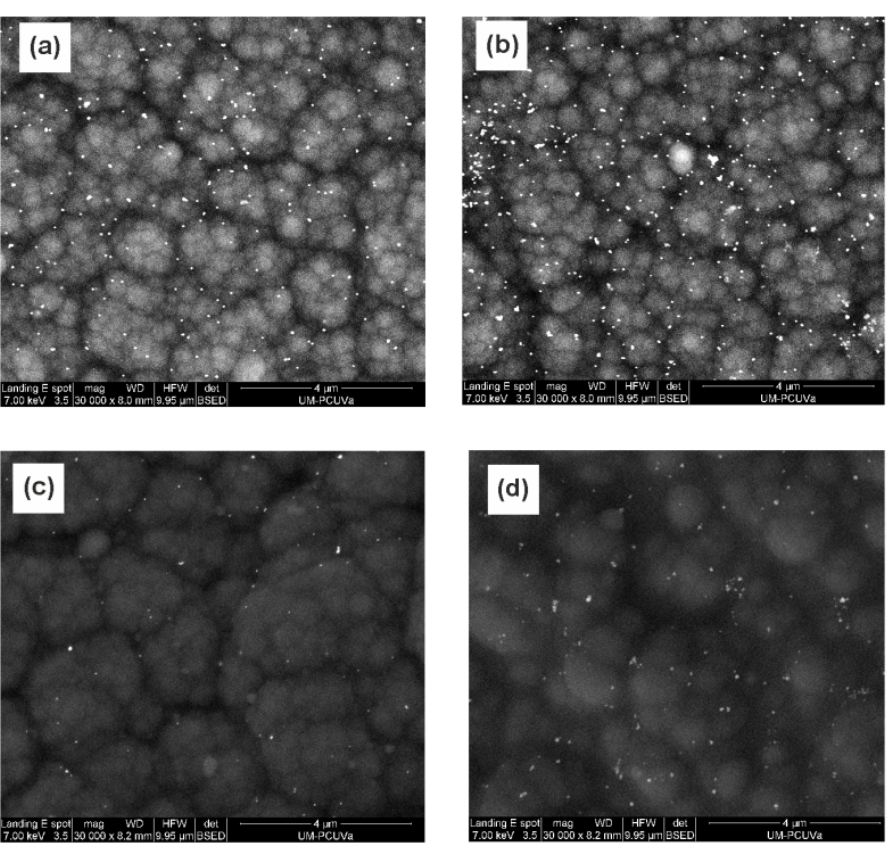

Figure 2: SEM images of Ppy/AuNP fims deposited on SS (a) Ppy/AuNP-T-CPss; (b) Ppy/AuNP-C-CPss; (c) Ppy/AuNP-T-CAss; (d) Ppy/AuNP-C-CAss.

incorporated in the Ppy films was higher when using CP than that when using CA. In turn, using cogeneration, the amount of nanoparticles incorporated was higher than using trapping

\section{Electrochemical impedance spectroscopy}

Electrochemical impedance spectroscopy (EIS) can provide information about the conductivity changes resulting from the insertion of AuNPs in the Ppy films. The complex impedance can be plotted as the real $\left(Z_{\text {real }}\right)$ vs imaginary $\left(Z_{\text {imaginary }}\right)$ components (Nyquist plot), which are related to the resistance and capacitance of the cell, respectively. At high frequencies (left part of the diagram) the semicircular part is associated to electron-transfer limited processes. The diameter of the semicircle is equivalent to the electron-transfer resistance $\left(R_{\mathrm{ct}}\right)$. The linear part that appears at lower frequencies is related to diffusion limited processes. In the case of Ppy deposited by CA, the Nyquist plot (Figure 3a) was a semicircle $\left(R_{\mathrm{ct}}, 45.54 \mathrm{k} \Omega\right)$. The electrochemical process was thus, dominated by electron transfer.

The insertion of AuNPs in the Ppy films clearly modified the electrical behavior. In effect, the Nyquist plot of Ppy/AuNP-T-CA $A_{P t}$ films obtained by trapping (Figure $3 b$ ) showed a semicircle with a smaller $R_{\mathrm{ct}}(13.52 \mathrm{k} \Omega)$ in the high frequencies region. At low frequencies a straight line with a slope of $45^{\circ}$ was observed indicating a contribution of both electron transfer and diffusion processes. In Ppy/AuNPs-C$\mathrm{CA}_{\mathrm{Pt}}$ films obtained by cogeneration $R_{\mathrm{ct}}$ was practically zero and only the linear part corresponding to diffusion control was observed (Figure 3c)

These results confirm the ability of AuNPs to reduce the resistance by facilitating the electron transfer. In fact, as observed in SEM images the number of AuNPs inserted in the films was higher using cogeneration, explaining the drastic decrease in the resistance. This is in good agreement with previous published results that indicated that the presence of AuNPs in the polymer matrix resulted in an increase in conductivity [31].

EIS results of Ppy/AuNPs films deposited by CP showed similar trends, but resistance and impedance values were clearly smaller than those observed in films deposited by CA. For instance, the impedance values of Ppy/AuNP-C-CP $\mathrm{Pt}_{\mathrm{t}}$ were one third smaller than those obtained by CA (Figure 3d). Again, the high number of AuNPs inserted in the nanocomposite by $\mathrm{CP}$, explains the improvement in the conductivity. 

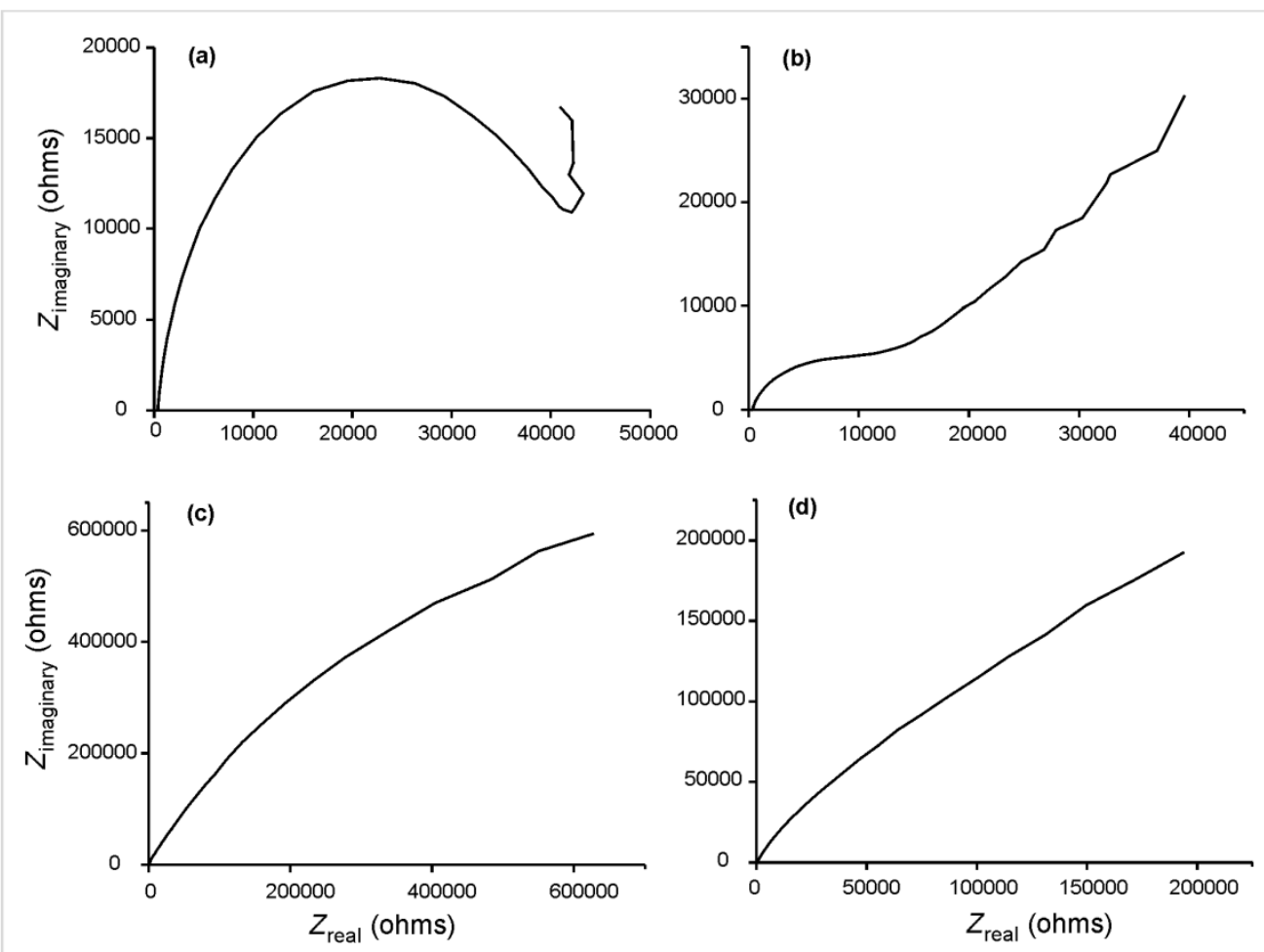

Figure 3: Nyquist plot of films deposited onto Pt registered in $0.1 \mathrm{mo} / \mathrm{L} \mathrm{KCl}$. Frequency swept from 105 to $0.1 \mathrm{~Hz}$ (a) Ppy-CApt; (b) Ppy/AuNP-T-CApt: (c) Ppy/AuNP-C-CApt; (d) Ppy/AuNP-C-CP Pt.

It is important to point out, that EIS measurements carried out in films deposited on SS by CA where irreproducible, indicating that the films obtained were unstable. Films deposited on SS by CP produced reproducible results but with higher $R_{\mathrm{ct}}$ and impedance values than those found on the platinum substrate. In fact, in the Nyquist plot for bare Ppy-CP $\mathrm{SS}_{\mathrm{SS}}$ the $R_{\mathrm{ct}}$ was so high that the semicircle was not completed.

According to these results, and taking into account that lower $R_{\mathrm{ct}}$ values correspond to an increase of the voltammetric signal [32] the cogeneration combined with chronopotentiometry seems to be the most suitable electrodeposition technique to prepare voltammetric sensors.

\section{Electrochemical behavior of Ppy/AuNPs prepared using different techniques}

The electrochemical behavior of Ppy and Ppy/AuNP films was analyzed using cyclic voltammetry in $0.1 \mathrm{~mol} / \mathrm{L} \mathrm{KCl}$ solution. The responses are influenced by the polymerization method, the deposition technique and the type of substrate. Before going into the details, it is important to notice that, in good accordance with previously published results, the first scan was always different from the subsequent cycles. Subsequent cycles were highly reproducible [22]. For this reason, in the next figures, the fifth scan will be displayed.

For Ppy films deposited on platinum using $\mathrm{CA}$ or $\mathrm{CP}$, the first cycle showed two redox processes corresponding to the polaron and bipolaron. In successive cycles one single process (anodic wave at $-0.35 \mathrm{~V}$ and the corresponding cathodic peak at around $-0.5 \mathrm{~V}$ ) was found. When deposition was carried out on SS, voltammograms showed lower intensities and in the case of Ppy-CA $\mathrm{AS}_{\mathrm{SS}}$, a certain irreproducibility.

When AuNPs were introduced in the films (Ppy/AuNPs), the preparation method induced important differences. In films deposited on platinum, the insertion of AuNPs caused an increase in the intensity of the peaks. Simultaneously the separation between the anodic and the cathodic waves was reduced. This is illustrated in Figure 4 for films deposited on Pt by CP. 
According to this, it can be concluded that the reversibility of the redox processes is improved in Ppy/AuNP composites. The increase was more pronounced in films deposited by $\mathrm{CP}$ than in films deposited by CA. As the number of AuNPs inserted in the films was higher in films deposited by CP (Figure 2), the electrocatalytic effect of the AuNPs is confirmed. This is also in agreement with EIS results that demonstrated that the insertion of AuNPs increased the conductivity.

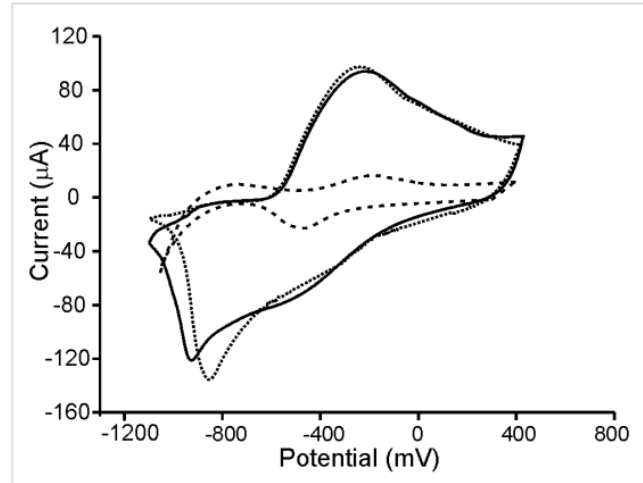

Figure 4: Cyclic voltammograms of $\mathrm{Ppy}-\mathrm{CP}_{\mathrm{Pt}}$ (dashed line), Ppy/AuNPs-T-CP Pt (dotted line) and Ppy/AuNPs-C-CP Pt $_{\text {(solid line) }}$ immersed in $0.1 \mathrm{~mol} / \mathrm{L} \mathrm{KCl}$. Scan rate $0.1 \mathrm{~V} / \mathrm{s}$. the substrate in the electrochemical properties of the films. The influence of the method used to introduce the AuNPs (trapping or cogeneration) was not so important. In fact, when films were deposited onto Pt, the differences in the voltammograms prepared by trapping or by cogeneration were minimal. In contrast, when SS was used as the substrate, the differences observed between trapping and cogeneration could be ascribed to the irreproducibility and therefore conclusions could not be deduced.

The irreproducibility observed in stainless steel can be clearly attributed to pitting processes produced by chloride ions. In consequence, reproducibility could be improved by changing the supporting electrolyte.

According to this idea, the influence of the supporting electrolyte was further investigated using phosphate buffer. As expected, the large size and high charge of the phosphate anions, made difficult the diffusion of anions inside the polymeric film producing a broadening of the peaks and the increase in the separation between the anodic and the cathodic waves that appeared at -0.15 and $-0.8 \mathrm{~V}$, respectively [33] . A part from the broadening of the peaks, the effects caused by AuNPs were similar to those observed in $\mathrm{KCl}$ (e.g., increase in the intensity of the peaks accompanied by a decrease in the separation between anodic and cathodic waves.

It is important to point out that, when the deposition was carried out on SS, a decrease in the intensity of the peaks accompanied by a separation between the anodic and cathodic waves was observed. This behavior pointed to the interference between SS and AuNPs. In addition, some irreproducibility was observed (as it also happened in EIS experiments).

In fact, a part from the differences already commented in the electrochemical behavior of Ppy/AuNps films deposited onto P and SS, the most remarkable difference was related to their stability and lifetime. We already mentioned that the first cycle was different from the subsequent ones, but the changes occurring in successive cycles were more pronounced in films deposited on stainless steel substrates. The variation coefficients calculated in films deposited on Pt by CP or CA were less than $2 \%$ and $5 \%$, respectively. The $\% \mathrm{CV}$ calculated from films deposited on SS were $8 \%$ for CP and $15-20 \%$ for CA. Moreover, when electrodes were withdrawn from the solution and reintroduced in the tested solution, electrodes deposited onto SS, changed completely their electrochemical response and could not be further used.

The above results established the important influence of the electropolymerization method (CA or $\mathrm{CP}$ ) and of the nature of
Using phosphate buffer, the pitting processes were avoided and the reproducibility of films deposited on SS was clearly improved and was similar to that calculated in films deposited on platinum (CV less than $5 \%$ ).

\section{Electrocatalytic and sensing behavior towards catechol}

Once stable Ppy/AuNP electrodes were obtained, their electrocatalytic and sensing properties towards catechol (a phenolic compound of interest in the food industry), were analyzed in terms of signal amplification and peak shifts. Experiments were carried out in the range between -0.1 and $0.8 \mathrm{~V}$ at a scan rate of $0.1 \mathrm{~V} / \mathrm{s}$ in phosphate buffer. Under these conditions, SS could be used as a substrate due to the absence of pitting processes. Notice also that the polaron-bipolaron response of pyrrole occurs out of this range at negative potentials.

Catechol produced the expected well-shaped redox pair generated by the two-electron oxidation/reduction of the ortho-dihydroquinone to benzoquinone [26]. The reversibility of the peaks was improved with the incorporation of the AuNPs. Simultaneously, the intensity of the peaks increased with the concentration of AuNPs. This is illustrated in Figure 5 for electrodes deposited on SS by CP. As observed in the Figure, the 
separation between the anodic and cathodic waves was $300 \mathrm{mV}$ in Ppy-CPss films and only $100 \mathrm{mV}$ in Ppy/AuNP-T-CP

These effects were stronger in films deposited by CP than in films deposited by $\mathrm{CA}$, due to the higher concentration of nanoparticles. In contrast, the method to insert the nanoparticles (trapping or cogeneration) only produced small changes in the intensities and positions of the peaks, probably due to the minimal differences in the AuNPs concentration.

The electrocatalytic effect was stronger in films deposited on platinum than in SS. This is in good accordance to previously published reports that have established that AuNPs exhibit a catalytic behavior when deposited onto platinum due to the synergy between both metals [34]

The effect of the concentration of catechol was studied by immersing the electrodes prepared by $\mathrm{CP}$ in $1 \cdot 10^{-5}$ to $1 \cdot 10^{-3} \mathrm{~mol} / \mathrm{L}$ catechol solutions. A linear increase in the intensity of the peaks with the concentration was observed in the studied range. The limit of detection (LOD) was calculated from the calibration curves following the " $3 \mathrm{sd} / \mathrm{m}$ " criterion. As observed in Table 2, the LODs were in the range from $10^{-5}$ to $10^{-6} \mathrm{~mol} / \mathrm{L}$. The LOD obtained using Ppy/AuNP composite films was almost one order of magnitude lower than the one observed in Ppy films. The synergy between platinum and AuNPs increased the sensitivity, allowing a further decrease in the LODs. This synergy is not so important when using SS. Therefore, the use of SS as a substrate, provides stable sensors with good LODs while decreasing the price of the devices considerably.

\section{Conclusion}

Ppy/AuNP nanocomposites have been successfully prepared employing in situ polymerization of pyrrole using tetrachloroauric acid as an oxidant in the presence of gold ions and by trapping AuNPs in a Ppy matrix during the electropolymerization. SEM images confirmed the formation of uniform nanocomposites on smooth platinum and stainless steel substrates.
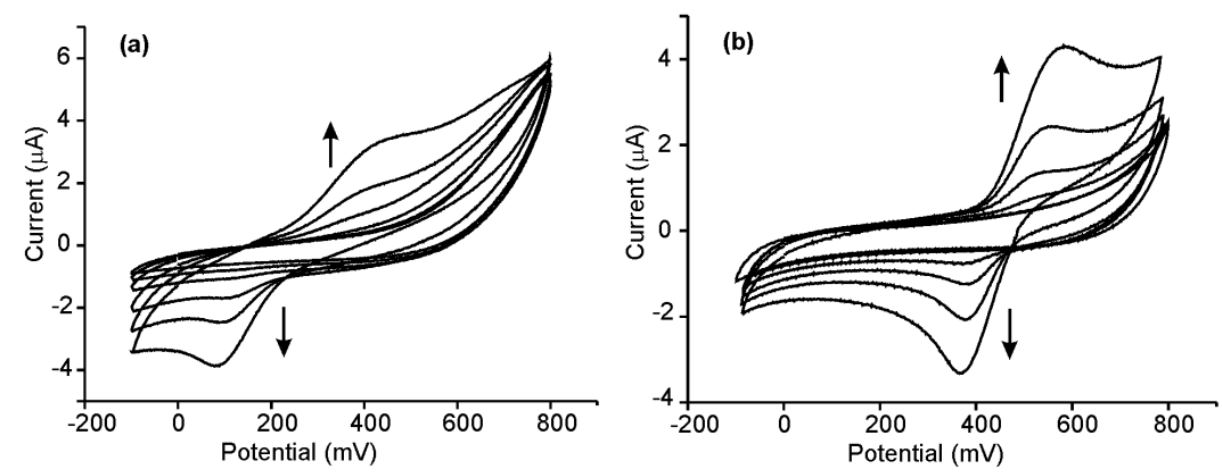

Figure 5: Voltammograms registered using electrodes deposited by CP on SS immersed in $1 \cdot 10^{-5}$ to $1 \cdot 10^{-3} \mathrm{~mol} / \mathrm{L}$ solutions of catechol in $0.01 \mathrm{~mol} / \mathrm{L}$ phosphate buffer (pH 7.0): (a) Ppy-CPSS (b) Ppy/AuNP-T-CPSS.

\begin{tabular}{|c|c|c|c|c|}
\hline Sensor & $\begin{array}{c}\text { LOD (mol/L) } \\
\text { (cathodic peak) }\end{array}$ & $\mathrm{R}^{2}$ & $\begin{array}{c}\text { LOD (mol/L) } \\
\text { (anodic peak) }\end{array}$ & $\mathrm{R}^{2}$ \\
\hline Ppy-CP $P_{p t}$ & $9.1 \cdot 10^{-5}$ & 0.977 & $5.3 \cdot 10^{-5}$ & 0.989 \\
\hline Ppy/AuNP-C-CP $P_{P t}$ & $2.4 \cdot 10^{-5}$ & 0.976 & $8.8 \cdot 10^{-5}$ & 0.996 \\
\hline Ppy/AuNP-T-CP Pt & $0.9 \cdot 10^{-5}$ & 0.984 & $0.3 \cdot 10^{-5}$ & 0.981 \\
\hline Ppy-CPss & $8.9 \cdot 10^{-5}$ & 0.956 & $7.2 \cdot 10^{-5}$ & 0.975 \\
\hline Ppy/AuNP-C-CPss & $4.3 \cdot 10^{-5}$ & 0.977 & $3.1 \cdot 10^{-5}$ & 0.971 \\
\hline Ppy/AuNP-T-CPSS & $3.2 \cdot 10^{-5}$ & 0.968 & $1.1 \cdot 10^{-5}$ & 0.975 \\
\hline
\end{tabular}


The presence of AuNPs in the polymer matrix resulted in an increase in the conductivity and in the intensity of the voltammetric signals. These variations in conductivity and intensity of voltammograms are directly related to the number of AuNPs inserted in the Ppy films.

Irreproducibility observed in the EIS and voltammetric measurements carried out in $\mathrm{KCl}$ using films deposited on stainless steel, caused by pitting process can be avoided by using phosphate buffer as supporting electrolyte.

As an electrochemical sensor, the Ppy/AuNP deposited on plat inum exhibited important electrocatalytic activity towards the oxidation of catechol. The effect was higher in films obtained by CP than in films obtained by CA. The influence of the method used to introduce the AuNPs (trapping or cogeneration) was not so important. The detection limits were in the range of $10^{-5}$ to $10^{-6} \mathrm{~mol} / \mathrm{L}$, which is lower than the concentration usually found in foods and beverages such as wines and musts. The synergy between Pt and Au nanoparticles gave rise to lowe LODs. In turn, stainless steel can be used as the substrate in the absence of $\mathrm{KCl}$, with a LOD only slightly higher than those obtained in sensors deposited on Pt, but at a lower cost.

\section{Experimental}

\section{Reagents and solutions}

All experiments were carried out in deionized Milli-Q water (Millipore, Bedford, MA). Pyrrole, tetrachloroauric acid, 1-decanesulfonic acid (DSA), potassium chloride, sodium phosphate, potassium phosphate and catechol were purchased from Sigma-Aldrich. Commercially available reagents and solvents were used without further purification. $10^{-3} \mathrm{~mol} / \mathrm{L}$ stock solutions of catechol were prepared by solving the corresponding compound in $\mathrm{KCl}$ solution $(0.1 \mathrm{~mol} / \mathrm{L})$ or phosphate buffer solution ( $\mathrm{pH} 7.0 ; 0.1 \mathrm{~mol} / \mathrm{L}$ ). Solutions with lower concentration were prepared from the stock solutions by dilution.

\section{Preparation of the Au colloidal suspension}

The synthesis of AuNPs colloids was carried out according to the procedure proposed by Slot and Geuze [35]. Two solutions were prepared: (1) $\mathrm{HAuCl}_{4}\left(0.25 \cdot 10^{-3} \mathrm{~mol} / \mathrm{L}\right)$ in deionized water and (2) sodium citrate dehydrate $\left(17 \cdot 10^{-3} \mathrm{~mol} / \mathrm{L}\right)$ in deionized water. $20 \mathrm{~mL}$ of solution (1) was heated until boiling on a hot plate, then $1 \mathrm{~mL}$ of solution (2) was quickly added to the $\mathrm{HAuCl}_{4}$ solution while stirring. The mixture was then boiled for 20 min. Using this procedure, a red colloid with a UV absorbance maximum at $\lambda=540 \mathrm{~nm}$ was obtained.

\section{Instruments}

Electropolymerizations and electrochemical studies were carried out at room temperature in an EG\&G Parstat 2273 potentiostat/galvanostat using a three-electrode configuration The same instrument was used for the EIS experiments. UV-vis spectra were recorded on a Shimadzu UV-2600 model spectrometer. A SEM-FEI (QUANTA 200F) was used to record the images of the electrode surfaces.

\section{Electropolymerization methods}

The auxiliary electrode was a conventional Pt electrode. The reference electrode was an $\mathrm{Ag} / \mathrm{AgCl}$ electrode in a $3 \mathrm{~mol} / \mathrm{L} \mathrm{KCl}$ solution. Pt and stainless steel 316L (SS) disks (1 mm diameter) were used as working electrodes. The disks were polished with $0.3 \mu \mathrm{m}$ alumina suspension using a microcloth polishing pad and rinsed with deionized water in an ultrasonic bath.

\section{Electropolymerization of Ppy films}

The Ppy films were obtained by electropolymerization from a solution containing $0.1 \mathrm{~mol} / \mathrm{L}$ pyrrole and $0.05 \mathrm{~mol} / \mathrm{L} 1$-decanesulfonic acid (DSA) using two electrochemical techniques: chronopotentiometry (CP) using a constant potential at $0.8 \mathrm{~V}$ over a period of $300 \mathrm{~s}$, and chronoamperometry (CA) using $0.02 \mathrm{~mA}$ over a period of $300 \mathrm{~s}$ (except otherwise indicated) Films were deposited onto Pt and SS.

\section{Electropolymerization of Ppy/AuNPs films}

Ppy/AuNPs films were obtained using two different approaches. On one hand, Ppy/AuNPs films were synthesized by the "trapping method" from a solution containing $0.2 \mathrm{~mol} / \mathrm{L}$ pyrrole, $0.1 \mathrm{~mol} / \mathrm{L}$ DSA. This solution was mixed (1:1) with a solution containing AuNPs previously formed (Au colloidal suspension). Films were polymerized by chronoamperometry using a constant potential at $0.8 \mathrm{~V}$ over a period of $300 \mathrm{~s}$, and by chronopotentiometry using $0.02 \mathrm{~mA}$ over a period of $300 \mathrm{~s}$. Sensors obtained by trapping were termed as Ppy/AuNP-T-CA (obtained by chronoamperometry) and Ppy/AuNP-T-CP (obtained by chronopotentiometry)

Ppy/AuNPs films were also synthesized using the "cogeneration method" by mixing a solution containing tetrachloroauric acid $10^{-3} \mathrm{~mol} / \mathrm{L}$ and a solution containing pyrrole and DSA. In this method, and according to the oxidation potentials of pyrrole (0.7 V vs SCE) and the reduction potential of $\mathrm{AuCl}_{4}{ }^{-}(1 \mathrm{~V})$, the AuNPs where generated in situ and inserted in the polymeric film during the electrochemical growth. Also in this case, electropolymerization was carried out by $\mathrm{CA}$ and $\mathrm{CP}$ under the same conditions used for trapping. Sensors obtained by cogeneration were termed as Ppy/AuNP-C-CA (obtained by chronoamperometry) and Ppy/AuNP-C-CP (obtained by chronopotentiometry)

In all cases, films were deposited onto Pt and SS disks. The type of substrate will be denoted using a subscript (i.e., Ppy/AuNPs- 
$\mathrm{C}-\mathrm{CP}_{\mathrm{Pt}}$ or Ppy/AuNPs-T-CP $\mathrm{Ss}$ ). Once prepared, the polymeric films were extracted from the generation solution and washed thoroughly with water.

\section{Electrochemical impedance spectroscopy (EIS) characterization}

EIS was performed in a $0.1 \mathrm{~mol} / \mathrm{L} \mathrm{KCl}$ solution with a frequency range from 105 to $0.1 \mathrm{~Hz}$ and a signal amplitude of $10 \mathrm{mV}$, at a working potential of $0.0 \mathrm{~V}$.

\section{Tests of the voltammetric sensors}

The Ppy and Ppy/AuNPs films were used as working electrodes in electrochemical experiments. The reference electrode was $\mathrm{Ag} / \mathrm{AgCl} / \mathrm{KCl} 3 \mathrm{~mol} / \mathrm{L}$ and the counter electrode was a platinum wire.

Cyclic voltammetry was carried out at room temperature with a scan rate of $0.1 \mathrm{~V} / \mathrm{s}$ in the potential range between $-1.0 \mathrm{~V}$ and $0.8 \mathrm{~V}$ (vs Ag/AgCl) except otherwise indicated.

Calibration curves were constructed from catechol solutions with concentrations ranging from $1 \cdot 10^{-5}$ to $1 \cdot 10^{-3} \mathrm{~mol} / \mathrm{L}$. The limits of detection (LODs) were calculated following the " $3 \mathrm{sd} / \mathrm{m}$ " criterion, where " $\mathrm{m}$ " is the slope of the calibration graph, and "sd" was estimated as the standard deviation $(n=5)$ of the voltammetric signals at the concentration level corresponding to the lowest concentration of the calibration plot $[36,37]$.

\section{Acknowledgements}

Financial support by the MINECO and FEDER (grant CICYTAGL2012-33535) and Junta de Castilla y León (VA-032U13) is gratefully acknowledged. CMP would also like to thank the University of Valladolid for the grant (PIF-UVa).

\section{References}

1. Ramanavičius, A.; Ramanavičienè, A.; Malinauskas, A. Electrochim. Acta 2006, 51, 6025-6037. doi:10.1016/.electacta.2005.11.052

2. Ates, M. Mater. Sci. Eng., C 2013, 33, 1853-1859. doi:10.1016/.msec.2013.01.035

3. Li, C. M.; Sun, C. Q.; Chen, W.; Pan, L. Surf. Coat. Technol. 2005, 198 474-477. doi:10.1016/j.surfcoat.2004.10.065

4. Chillawar, R. R.; Tadi, K. K.; Motghare, R. V. J. Anal. Chem. 2015, 70, 399-418. doi:10.1134/S1061934815040152

5. Yoon, H. Nanomaterials 2013, 3, 524-549. doi:10.3390/nano3030524

6. Saha, K.; Agasti, S. S.; Kim, C.; Li, X.; Rotello, V. M. Chem. Rev. 2012 112, 2739-2779. doi:10.1021/cr2001178

7. Lin, X.; Ni, Y.; Kokot, S. Anal. Chim. Acta 2013, 765, 54-62. doi:10.1016/.aca.2012.12.036
8. Valcárcel, M.; López-Lorente, A. I. Gold Nanoparticles in Analytical Chemistry. In Comprehensive Analytical Chemistry (Wilson and Wilson's); Barcelo, D., Ed.; Elsevier: Amsterdam, NL, 2014; Vol. 66 pp 1-601.

9. Jiang, H.; Moon, K.-S.; Li, Y.; Wong, C. P. Chem. Mater. 2006, 18 2969-2973. doi: $10.1021 / \mathrm{cm} 0527773$

10. Pogulyaichenco, N. A.; Hui, S.; Malev, V. V.; Kondratiev, V. V. Russ. J. Electrochem. 2009, 45, 1176-1182. doi:10.1134/S1023193509100103

11.Zhang, Z.; Wang, F.; Chen, F.; Shi, G. Mater. Lett. 2006, 60, 1039-1042. doi:10.1016/j.matlet.2005.10.071

12.Zanardi, C.; Terzi, F.; Pigani, L.; Heras, A.; Colina, A.; Lopez-Palacios, J.; Seeber, R. Electrochim. Acta 2008, 53, 3916-3923. doi:10.1016/j.electacta.2007.07.057

13.Spain, E.; Keyes, T. E.; Forster, R. J. Electrochim. Acta 2013, 109 102-109. doi:10.1016/j.electacta.2013.07.018

14.Zhang, J.; Liu, X.; Wu, S.; Xu, H.; Cao, B. Sens. Actuators, B 2013, 186, 695-700. doi:10.1016/j.snb.2013.06.063

15. Rezaei, B.; Boroujeni, M. K.; Ensafi, A. A. Biosens. Bioelectron. 2014, 60, 77-83. doi:10.1016/.j.bios.2014.03.028

16. Li, J.; Xie, H.; Chen, L. Sens. Actuators, B 2011, 153, 239-245. doi:10.1016/j.snb.2010.10.040

17.Rapecki, T.; Donten, M.; Stojek, Z. Electrochem. Commun. 2010, 12 , 624-627. doi:10.1016/j.elecom.2010.02.015

18. Malinauskas, A.; Malinauskiene, J.; Ramanavičius, A. Nanotechnology 2005, 16, 51-62. doi:10.1088/0957-4484/16/10/R01

19.Chen, W.; Li, C.-M.; Yu, L.; Lu, Z.; Zhou, Q. Electrochem. Commun 2008, 10, 1340-1343. doi:10.1016/j.elecom.2008.07.001

20.Heinig, N. F.; Kharbanda, N.; Pynenburg, M. R.; Zhou, X. J.; Schultz, G. A.; Leung, K. T. Mater. Lett. 2008, 62, 2285-2288. doi:10.1016/.matlet.2007.11.094

21. Razavipanah, I.; Rounaghi, G. H.; Zavvar, M. H. A. Anal. Lett. 2014, 47, 117-133. doi:10.1080/00032719.2013.832277

22. He, Y.; Yuan, J.; Shi, G.; Wu, P. Mater. Chem. Phys. 2006, 99, 253-257. doi:10.1016/j.matchemphys.2005.10.033

23. Hurtado-Fernández, E.; Gómez-Romero, M.; Carrasco-Pancorbo, A.; Fernández-Gutiérrez, A. J. Pharm. Biomed. Anal. 2010, 53 1130-1160. doi:10.1016/j.jpba.2010.07.028

24.Makhotkina, O.; Kilmartin, P. A. Anal. Chim. Acta 2010, 668, 155-165. doi:10.1016j.aca.2010.03.064

25. Brett, A. M. O.; Ghica, M.-E. Electroanalysis 2003, 15, 1745-1750. doi:10.1002/elan.200302800

26. Kilmartin, P. A.; Zou, H.; Waterhous, A. L. Am. J. Enol. Vitic. 2002, 53, 294-302.

27.Parra, V.; Hernando, T.; Rodríguez-Méndez, M. L.; de Saja, J. A. Electrochim. Acta 2004, 49, 5177-5185. doi:10.1016/j.electacta.2004.06.031

28.Medina-Plaza, C.; Furini, L. N.; Constantino, C. J. L.; de Saja, J. A.; Rodríguez-Mendez, M. L. Anal. Chim. Acta 2014, 851, 95-102. doi:10.1016/j.aca.2014.08.049

29. Arrieta Almario, A. A.; Vieira, R. L. J. Chil. Chem. Soc. 2006, 51 , 971-974. doi:10.4067/S0717-97072006000300009

30. Arrieta Almario, A. A.; Tarazona Caceres, R. L. J. Chil. Chem. Soc. 2009, 54, 14-19. doi:10.4067/S0717-97072009000100004

31. Singh, S.; Jain, D. V. S.; Singla, M. L. Anal. Methods 2013, 5, 1024-1032. doi:10.1039/c2ay26201k

32. Di Carlo, G.; Curulli, A.; Trani, A.; Zane, D.; Ingo, G. M. Sens. Actuators, $B$ 2014, 191, 703-710. doi:10.1016/.snb.2013.10.063 


\section{Chapter 3: Results and discussion}

Paper IV: Electrochemical behavior of polypyrrol/AuNP composites deposited by different electrochemical methods: sensing properties towards catechol.

Beilstein J. Nanctechnol 2015, 6, 2052-2061.

33. Ratautaite, V.; Ramanaviciene, A.; Oztekin, Y.; Voronovic, J.;

Balevicius, Z.; Mikoliunaite, L.; Ramanavicius, A. Colloids Surf., A 2013, 418, 16-21. doi:10.1016/. colsurfa. 2012.10.052

34. Xiao, F.; Zhao, F,; Zhang, Y.; Guo, G.; Zeng, B. $\alpha$. Phys. Chem. C

2009, 113, 849-855. doi:10.1021/p808162g

35. Slot, J. W.; Geuze, H. J. Eur. J. Cell Biol. 1985, 38, 87-93.

36. IUPAC Analytical Chemistry Division. Pure Appl. Chem. 1976, 45, 99-103. doi:10.1351/pac197645020099

37. Medina-Plaza, C.; Garcia-Cabezón, C.; García-Hernández, C.

Bramorski, C.; Blanco-Val, Y.; Martin-Pedrosa, F.; Kawai, T.;

de Saja, J. A.; Rodríguez-Méndez, M. L. Anal, Chim. Acta 2015, 853 ,

572-578. doi:10.1016/j.aca.2014.10.046

\section{License and Terms}

This is an Open Access article under the terms of the

Creative Commons Attribution License

(http://creativecommons.org/licenses/by/2.0), which

permits unrestricted use, distribution, and reproduction in

any medium, provided the original work is properly cited.

The license is subject to the Beilstein.Journal of

Nanotechnology terms and conditions:

(http://www.beilstein-journals.org/bjnano)

The definitive version of this article is the electronic one

which can be found at:

doi:10.3762/bjnano.6.209 


\subsection{Paper V}

Analysis of musts and wines by means of a bio-electronic tongue based on tyrosinase and glucose oxidase using polypyrrole/gold nanoparticles as the electron mediator.

By Celia García-Hernández, Cristina García-Cabezón, Fernando MartínPedrosa and María Luz Rodríguez-Méndez.

Food Chemistry 289 (2019) 751-756.

(doi:10.1016/j.foodchem.2019.03.107) 


\subsubsection{Motivation and objective}

With the aim of elaborating a wine of good quality, it is very important for vineyard growers to harvest grapes at the optimal point of ripeness, the point at which the grapes have reached an appropriate phenolic maturation. As the grapes ripen, many chemical, physical and microbiological processes may occur that can strongly influence the quality of the future elaborated wines. Moreover, wine quality will also depend on many other factors, such as grape variety, wine characteristics, weather factors or viticulture practices.

In recent years, multi-parametric methods have been applied in the food industry for quality control purposes. Infrared spectroscopy (FTIR, NIR) combined with chemometric methods is emerging as a useful technique to analyze grapes and wines. It is rapid, versatile and requires minimal sample preparation. For instance, sugar content or acidity has been analyzed by means of these methods. In addition, the polyphenolic and antioxidant content in wine samples can be assessed by other recognized traditional spectrophotometric methods, such as Total Polypneol index (TPI), FRAP, DPPH, ORAC, and Folin-Ciocalteu Indexes, among others. However, up to the present time, a single method has not been recognized as the most adequate, and the results obtained depend on the method used. Therefore, the recommendation when approaching the study of the antioxidant activity of wines is to use more than one method.

On this matter, electronic and bioelectronic tongues based on electrochemical sensors have been developed and used in food quality control. In this sense, electrochemical techniques can represent an advantage thanks to their higher sensitivity and relatively low cost in comparison with the spectroscopic methods. However, experiments with ETs and bioETs provide a vast amount of data that must be analyzed with the appropriate multiparametric statistical tools. So it is very important to select statistical studies that allow us to extract the maximum information from each electrochemical analysis carried out with ETs/bioETs.

In the case of wines and grapes, there are several research studies that have applied the technology of electronic tongues to analyze parameters of interest in terms of quality control. The UVaSens group is one of the most active research groups that have implemented ETs and bioETs in this field. However, up to the present time, no research work in the field of grapes and wines has implemented an electronic tongue able to assess the quality of wines from the data 
obtained when analyzing the grapes that will be used to elaborate those wines and this is the proposed objective for this work.

In this work, we have developed an electrochemical bioelectronic tongue based on Ppy and Ppy/AuNP films with the enzymes tyrosinase and glucose oxidase to analyze red grapes and their corresponding elaborated wines. The Ppy based-sensors were reported in a previous work, selecting the chronopotentiometry as the electrodeposition method, incorporating the AuNPs in situ during the electrodeposition using tetreacloroauric acid as precursor, and the use of two substrates, platinum and stain steel, to increase the cross-selectivity of the responses. In this case the counterion used was dodecylbenzenesulphonic acid (DBSA) instead of DSA, which acted in a similar way, but at a lower price. The results obtained from the electrochemical responses evidenced that the conducting polymer and AuNPs are effective electron mediators to be used with enzymes. Principal component analysis (PCA) and parallel factor analysis (PARAFAC) were used to discriminate samples of grapes with different sugar and polyphenolic contents and wines with different alcoholic degrees and polyphenolic content. Moreover, partial least squares (PLS-1) was capable of constructing regression models with good correlations between the data obtained with the bioET and the chemical parameters obtained by traditional methods. Further, using support vector machines (SVM), we were able to predict the alcoholic degree and polyphenolic content of the wines from the data obtained from red grapes with the bioET.

This result is of great interest for the food industry as it opens up the possibility of predicting some of the characteristics of the final wine from the beginning of the vinification process, allowing us to take actions to obtain high quality wines.

\subsubsection{Summary of the research work}

Biosensors inspired by sensors of Ppy and Ppy/AuNPs, reported before, were prepared. For this purpose, we selected the experimental conditions that improved the electrochemical responses of Ppy and Ppy/AuNP sensors. Therefore, the bioET consisted of eight biosensors of Ppy and Ppy/AuNP films prepared onto substrates of Pt and SS by chronopotentiometry. AuNPS were formed and introduced in the polymeric matrix during the electrodeposition experiments (previously mentioned as the cogeneration method). The average size of the as-prepared AuNPs 
has already been studied by SEM, resulting in about 30-40 nm. The enzymes, tyrosinase (Tyr) and glucose oxidase (GOx), were immobilized onto the sensor surfaces by casting the enzyme solutions and then cross-linking with glutaraldehyde. Tyrosinase is a phenoloxidase specific for the detection of phenols, whereas glucose oxidase is used for the detection of sugars.

Red grapes and their corresponding elaborated red wines were provided by the vineyards Bodega Cooperativa de Cigales and the Instituto Tecnológico Agrario de Castilla y León (ITACyL), both located in the Castilla y León region. The varieties of grapes analyzed were: Cabernet, Garnacha, Tempranillo, Juan García, Mencía Secano, Mencía Regadío, Prieto Picudo y Rufete. The Oenological Centre of Castilla y León analyzed the chemical parameters of the grapes and wines. The Brix degree and TPI of grapes and the alcoholic degree and TPI of wines were provided.

Grapes and wines were analyzed by cyclic voltammetry. The electrochemical responses of musts showed anodic peaks at positive potentials whereas, in the case of wines, the responses showed a redox process. However, voltammograms responses depended on the type of substrate (Pt or SS), the modifier (Ppy or Ppy/AuNP) and enzyme (Tyr or GOx). For instance, in the case of using platinum substrate, the voltammograms in wines showed a redox pair at negative potentials whereas, by using SS, the redox peak appeared with an anodic peak at the positive potential and the cathodic peak at the negative potentials, so the responses were more irreversible than for Pt substrates. Moreover, the responses in wines and musts were completely dissimilar, reflecting the fact that the composition of samples is different due to the concentration of ionic and redox species (e.g., polyphenols), which affects the interchange of ions in and out of the polymeric matrix during the redox process. The enzymatic activity of enzymes allowed us to obtain an important degree of selectivity in the responses, especially in the intensities and positions of the cathodic waves. Ppy/AuNPs and platinum substrate evidenced an efficient electron tranfer performance, increasing the intensities of the voltammetric responses. In conclusion, the responses showed a high degree of cross-selectivity.

Further, the voltammetric responses were used as input data for several statistical treatments. On the one hand, we used two different discrimination methods on the results, PCA and PARAFAC. The PCA score plots for wines showed samples located in space depending on their alcoholic degree and TPI values. In the case of musts, we were not able to discriminate the grapes according to their Brix degree or TPI value; however, samples were located in space in a 
similar way to that in which wines were located in the PCA score plot. This result indicates that the bioET might be able to establish interesting correlations between the grapes and wines.

With PARAFAC analysis, we gave a three-way nature to the date. The PARAFAC results for wines corroborate those obtained by the two-way PCA method; however, in the case of musts, the samples appeared to be discriminated according to their Brix degree. The PARAFAC analysis showed an important difference with respect to PCA, since we can obtain further information by analyzing the Mode plots obtained in PARAFAC. Therefore, Mode 1 gave information about the contribution of the samples, Mode 2 about the contribution of kernels (a reduction of variables method used to extract the information from voltammograms) and Mode 3 about the contribution of the sensors and their cross-selectivity.

On the other hand, we established correlations between the voltammograms obtained from the samples and the chemical composition. Using PLS-1, we obtained regressions models that correlated the data obtained in must and wines with their chemical parameters measured by traditional methods. The data obtained from musts were well correlated with the TPI and Brix degree, with only 3 components with low errors; however, the correlation coefficient obtained for the TPI results were lower than 0.90 . In the case of the wines, the voltammetric data also correlated with the TPI and alcoholic degree, also with 3 components, low errors, while the correlation coefficients were higher than in the case of must.

Finally, in order to predict values of TPI and alcoholic degree in wines, we carried out a supervised statistical method called support vector machine regression (SVMR). In this analysis, we introduced the voltammetric data obtained from musts with the bioET as the X-matrix and the chemical parameters of the wines elaborated from those musts as the $Y$-matrix. We then created correlation models to obtain a predictor model, after which, by applying the corresponding predictor model, we were able to predict values of the TPI and alcoholic degree in wines with low relative errors from the data obtained in the must with the bioET. This means that, from the data obtained in must, we are able to predict two chemical parameters that point to the quality of wines before having elaborated them. 

Paper V: Analysis of musts and wines by means of a bio-electronic tongue based on tyrosinase and glucose oxidase using polypyrrole/gold nanoparticles as the electron mediator.

\title{
Analysis of musts and wines by means of a bio-electronic tongue based on tyrosinase and glucose oxidase using polypyrrole/gold nanoparticles as the electron mediator
}

\author{
C. Garcia-Hernandez ${ }^{\mathrm{a}}$, C. Garcia-Cabezon ${ }^{\mathrm{a}}$, F. Martin-Pedrosa ${ }^{\mathrm{a}}$, M.L. Rodriguez-Mendez ${ }^{\mathrm{a}, *}$
}

a Group UVaSens, Engineers School, Universidad de Valladolid, 47011 Valladolid, Spain

\begin{tabular}{ll}
\hline A R T I C L E I N F O & A B S T R A C T \\
\hline $\begin{array}{l}\text { Keywords: } \\
\text { Electronic tongue } \\
\text { Bioelectronic tongue }\end{array}$ & $\begin{array}{l}\text { A bioelectronic tongue (bioET) based on combinations of enzymes (tyrosinase and glucose oxidase) and poly- } \\
\text { pyrrole (Ppy) or polypyrrole/AuNP (Ppy/AuNP) composites was build up and applied to the analysis and dis- } \\
\text { crimination of musts and wines. Voltammetric responses of the array of sensors demonstrated the effectiveness of } \\
\text { polypyrrole }\end{array}$ \\
$\begin{array}{l}\text { Gold nanoparticle } \\
\text { Must }\end{array}$ & $\begin{array}{l}\text { Using Principal Component Analysis and Parallel Factor Analysis it was possible to discriminate musts according } \\
\text { to the }{ }^{\circ} \text { Brix and TPI (Total Polyphenol Index), and wines according to the alcoholic degree and TPI. Partial Least }\end{array}$ \\
Sine & $\begin{array}{l}\text { Squares provided good correlations between the bioET output and traditional chemical parameters. Moreover, } \\
\text { Support Vector Machines permitted to predict the TPI and the alcoholic degree of wines, from data provided by } \\
\text { the bioET in the corresponding grapes. This result opens the possibility to predict wine characteristics from the } \\
\text { beginning of the vinification process. }\end{array}$
\end{tabular}

\section{Introduction}

During the last years, multi-parametric techniques are gaining interest in oenological applications because they make possible to assess many parameters in a single experiment. The most commonly used techniques are Near Infrared, FTIR or NMR which have been used to determine sugar content, acidity or sulfurose content, among other parameters (Buratti et al., 2011; Friedel, Patz, \& Dietrich, 2013; Hayasaka, Black, Hack, \& Smith, 2017; Musingarabwi, Nieuwoudt, Young, Eyéghè-Bickong, \& Vivier, 2015). Electronic tongues (ETs) are also multi-parametric systems that combine a multisensor system formed by a number of non-selective sensors with chemometric tools (Baldeón et al., 2015; Lvova et al., 2016; Rodríguez-Méndez, 2016; Peris \& Escuder-Gilabert, 2016; Smyth \& Cozzolino, 2013). ETs based on voltammetric sensors have been successfully used to analyze wines at different levels (fermentation, aging, variety of grape or fraudulences among many others) (Apetrei et al., 2012; Cetó et al., 2017; Gil-Sánchez et al., 2011; Giménez-Gómez et al., 2016; Pigani et al., 2011; Prieto et al., 2011; Rudnitskaya, Rocha, Legin, Pereira, \& Marques, 2010; Rodríguez-Méndez et al., 2014). Some works have also been dedicated to the analysis of grapes and their phenolic maturity (Medina-Plaza et al., 2016). However, there are no reports on the capability of an ET to establish correlations or predictions between the characteristics of grapes and that of wines elaborated from them. Such a difficult task requires the development of an improved electronic tongue with enhanced capabilities. In order to improve the sensitivity and cross-selectivity of the sensors, a possible strategy is to introduce bioelectrochemical sensors, to form the so-called bioelectronic tongues (bioET). BioETs combine the advantages of classical ETs, which provide overall information about the sample, with the specificity induced by biosensors (Ghasemi-Varnamkhasti et al., 2012; Medina-Plaza et al., 2016; Toko, 2013; Zeravik, Hlavacek, Lacina, \& Skládal, 2009). BioETs dedicated to wines include phenoloxidases specific for the detection of phenols (e.g. tyrosinase, laccase or peroxidase) and enzymes specific for the detection of sugars (glucose oxidase or fructose dehydrogenase) (Cetó, Capdevila, Mínguez, \& del Valle, 2014; Gutiérrez-Capitán et al. 2014; Medina-Plaza et al., 2015). However, in these works, the enzymatic activity is not very high and the peaks corresponding to the enzymatic process are weak.

In order to enhance the enzymatic signals, enzymes must be combined with appropriate electron mediators. Conducting polymers or gold nanoparticles have demonstrated to be excellent electron mediators in biosensors (Apetrei \& Apetrei, 2013; Mavrikou, Flampouri, Iconomou, \& Kintzios, 2017). In previous works, we have demonstrated

* Corresponding author at: Group UVASENS, Escuela de Ingenierías Industriales, Paseo del Cauce, 59, 47011 Valladolid, Spain.

E-mail addresses: celia.garcia.hernandez@uva.es (C. Garcia-Hernandez), crigar@eii.uva.es (C. Garcia-Cabezon), fmp@eii.uva.es (F. Martin-Pedrosa), mluz@eii.uva.es (M.L. Rodriguez-Mendez).

https://doi.org/10.1016/j.foodchem.2019.03.107

Received 31 October 2017; Received in revised form 5 March 2019; Accepted 20 March 2019

Available online 22 March 2019

0308-8146/ (c) 2019 Elsevier Ltd. All rights reserved. 
that phthalocyanines and AuNPs combined with conducting polymers improved electrochemical responses in sensors and biosensors for phenols analysis (García-Hernández et al., 2015; García-Hernández, García-Cabezón, Martin-Pedrosa, De Saja, \& Rodríguez-Méndez, 2016). Moreover, electropolymerization of conducting polymers onto electrode surfaces accompanied by in situ synthesis of AuNPs offers an easy way to fabricate composites combining both electron mediators that further will facilitate transfer kinetics process.

In this work, a bioET dedicated to the analysis of grapes and wines has been build up. For this purpose, an array of biosensors containing enzymes (glucose oxidase and tyrosinase) specialized in the detection of two of the main components usually analyzed to establish the quality of grapes and wines (glucose and phenols) was developed. The multisensor system was formed by tyrosinase or glucose oxidase biosensors containing polypyrrole (Ppy) and polypyrrole/AuNP composites (Ppy/ AuNP) as electron mediators. In order to further increase the cross-selectivity, sensors were prepared in two different substrates, platinum (Pt) and Stainless Steel (SS).

The bioET was used to establish correlations between the quality of grapes and the characteristics of the wines obtained from those grapes. Different chemometrics tools -Principal Component Analysis (PCA), Parallel Factor Analysis (PARAFAC), Partial Least Squares regression (PLS-1) and Support Vector Machine (SVM) were used to discriminate between grape juices (musts) and wines elaborated from different varieties of grapes, to establish correlations between the results obtained from the electronic tongue and the chemical parameters obtained using classical chemical methods and to predict the quality of the wines from the data obtained in musts.

\section{Materials and methods}

\subsection{Reagents and solutions}

Pyrrole, dodecylbenzenesulfonic acid sodium salt (DBSA), tetrachloroauric acid and potassium chloride were purchased from SigmaAldrich (St. Louis, MO, USA). Solvents were of reagent grade and used as supplied. Solutions were prepared by dissolving substances in deionized water (resistivity of $18.2 \mathrm{M} \Omega \cdot \mathrm{cm}^{-1}$ ) obtained from a Milli-Q system (Millipore, Billerica, MA, USA).

Glucose oxidase (GOx) (from Aspergillus niger, type VII, activity $\geq 100 \mathrm{U} \cdot \mathrm{mg}^{-1}$ ) and tyrosinase (Tyr) (from mushroom, activity $\geq 1000 \mathrm{U} \cdot \mathrm{mg}^{-1}$ ) were purchased from Sigma-Aldrich. $5 \mathrm{mg} \cdot \mathrm{mL}^{-1}$ solutions of enzymes were prepared in buffer phosphate $0.01 \mathrm{~mol} \cdot \mathrm{L}^{-1}(\mathrm{pH}$ 7.0).

\subsection{Grapes, musts and wines samples}

Red grapes were harvested in September 2014 from the vineyards "Bodega Cooperativa de Cigales" and "Instituto Tecnológico Agrario de Castilla y León (ITACyL)", both located in Castilla y León area (Valladolid, Spain). Varieties under study were Cabernet, Garnacha, Tempranillo, Juan García, Mencía Secano, Mencía Regadío, Prieto Picudo and Rufete.

Musts were prepared by crushing 200 berries during one minute. Then, musts were fermented and bottled. Musts and the corresponding wines elaborated from these musts were analyzed in The Oenological Centre of Castilla y León (Valladolid, Spain). Brix degree, alcoholic degree and the Total Polyphenol Index (TPI) were analyzed following international regulations (International Organisation of Vine and Wine, 2011). Results are collected in Table 1.

\subsection{Preparation of the electrochemical sensors and biosensors}

The bioET consisted of an array of eight electrodes based on Polypyrrole (Ppy) films doped with dodecylbenzenesulphonic acid (DBSA) obtained by electropolymerization on both platinum (Pt) and
Table 1

Average chemical parameters measured in musts and wines following international procedures.

\begin{tabular}{|c|c|c|c|c|c|}
\hline \multirow[b]{2}{*}{ Sample \# } & \multirow[b]{2}{*}{ Variety } & \multicolumn{2}{|l|}{ Musts } & \multicolumn{2}{|l|}{ Wines } \\
\hline & & ${ }^{\circ}$ Brix & TPI & `Alcoholic & TPI \\
\hline 1 & Cabernet & $22.4 \pm 2$ & $24 \pm 3$ & $13.0 \pm 0.8$ & $57 \pm 3$ \\
\hline 2 & Garnacha & $20.2 \pm 2$ & $17 \pm 2$ & $11.4 \pm 0.8$ & $41 \pm 3$ \\
\hline 3 & Juan García & $19.8 \pm 2$ & $24 \pm 2$ & $11.4 \pm 0.8$ & $41 \pm 3$ \\
\hline 4 & Mencía Regadío & $21.9 \pm 2$ & $19 \pm 2$ & $12.0 \pm 0.8$ & $44 \pm 3$ \\
\hline 5 & Mencía Secano & $22.7 \pm 2$ & $19 \pm 2$ & $12.8 \pm 0.8$ & $54 \pm 3$ \\
\hline 6 & Prieto Picudo & $22.0 \pm 2$ & $26 \pm 2$ & $12.5 \pm 0.8$ & $46 \pm 3$ \\
\hline 7 & Tempranillo & $22.8 \pm 2$ & $24 \pm 2$ & $13.3 \pm 0.8$ & $53 \pm 3$ \\
\hline 8 & Rufete & $20.2 \pm 2$ & $27 \pm 2$ & $11.1 \pm 0.8$ & $39 \pm 3$ \\
\hline
\end{tabular}

stainless steel 316L (SS) substrates (García-Hernández et al., 2015). Electropolymerizations and voltammetric measurements were carried out using a Parstat 2273 potentiostat/galvanostat (EG\&G, Oak Ridge, TN, USA).

$\mathrm{Pt}$ and SS disks (1 mm diameter) were used as working electrodes, the reference electrode was $\mathrm{Ag} \mid \mathrm{AgCl} / \mathrm{KCl} 3 \mathrm{~mol} \cdot \mathrm{L}^{-1}$ and the counter electrode was a platinum sheet with a surface of $1 \mathrm{~cm}^{2}$. The disks were polished with $0.3 \mu \mathrm{m}$ alumina suspension using a microcloth polishing pad and rinsed with deionized water in an ultrasonic bath.

The Ppy films were deposited onto the electrode surface from a solution containing $0.2 \mathrm{~mol} \cdot \mathrm{L}^{-1}$ pyrrole and $0.1 \mathrm{~mol} \cdot \mathrm{L}^{-1}$ DBSA using chronopotentiometry (CP) with a constant current of $0.02 \mathrm{~mA} \cdot \mathrm{cm}^{-2}$ during $300 \mathrm{~s}$. In the case of Ppy/AuNP composites, AuNPs were generated in situ and inserted in the polymeric film during polymerization and growth, using the pyrrole-DBSA solution mixed 1:1 with a solution containing $10^{-3} \mathrm{~mol} \cdot \mathrm{L}^{-1}$ tetrachloroauric acid and applying the same experimental conditions than for Ppy films $\left(0.02 \mathrm{~mA} \cdot \mathrm{cm}^{-2}\right.$ during $300 \mathrm{~s}$ ) Once prepared, films were washed thoroughly with deionized water. Morphology and size of the AuNPs in the as-preapared Ppy/ AuNP films have been studied by scanning electron microscopy (SEM). As expected, the average size of the AuNPs was about 30-40 nm and the nanoparticles were uniformly dispersed in the polymeric matrix showing a spherical shape. (García-Hernández et al., 2015).

Biosensors were prepared by depositing the enzymes GOx or Tyr onto the Ppy and Ppy/AuNP films by casting $5 \mu \mathrm{L}$ of $0.01 \mathrm{~mol} \cdot \mathrm{L}^{-1}$ phosphate buffer ( $\mathrm{pH} 7.0$ ) containing $5 \mathrm{mg} \cdot \mathrm{mL}^{-1}$ of the corresponding enzyme. After drying at room temperature, the process was repeated. Then, biosensors were immersed in glutaraldehyde $(2.5 \% \mathrm{v} / \mathrm{v}$, buffer solution) for $5 \mathrm{~min}$ and dried in air at room temperature. The biosensors were then rinsed with phosphate buffer to remove any unbound enzyme and stored at $4{ }^{\circ} \mathrm{C}$

Using these procedures Ppy-GOx, Ppy/AuNP-GOx, Ppy-Tyr and Ppy/AuNP-Tyr biosensors were prepared onto two types of substrates, platinum (Pt) and stainless steel (SS), obtaining thus, an array formed by eight sensors.

\subsection{Voltammetric measurements}

Voltammetric measurements were carried out in a three conventional cell using the sensors and biosensors as working electrodes, the reference electrode and the counter electrode were the same as in the case of the polymerization procedure. Cyclic voltammetry was carried out from $-1.0 \mathrm{~V}$ to $+0.8 \mathrm{~V}$ (vs Ag $\mid \mathrm{AgCl}$ ) at a scan rate of $0.1 \mathrm{~V} \cdot \mathrm{s}^{-1}$ Musts and wines were diluted 1:1 in potassium chloride solution $\left(0.3 \mathrm{~mol} \cdot \mathrm{L}^{-1}\right)$. Four replicas per sample were carried out (16 samples $\times 4$ repetitions $=64$ samples)

\subsection{Chemometrics}

Voltammograms were normalized and pre-processed with an adaptation of a data reduction technique based on 'kernels' (Gutiérrez- 
Osuna \& Nagle, 1999; Prieto et al., 2011). Using this method, voltammograms were "sliced" in 10 variables that were used as the input for statistical analysis. Thus, the array provided 80 data from each sample (8 sensors $\times 10$ kernels).

The statistical analysis was performed by using Matlab v2014b (The Mathworks Inc., Natick, MA, USA) and The Unscrambler v9.7. (CAMO Software AS, Oslo, Norway). Two non-supervised multivariate methods, Parallel Factor Analysis (PARAFAC) and Principal Component Analysis (PCA) were used to evaluate the discrimination capability of the electronic tongue and to compare the results obtained in grapes and wines. Partial Least Square Regression-1 (PLS-1) was used to establish correlations between the results obtained from the bioET and the chemical parameters given by the Oenological Centre of Castilla y León (Valladolid, Spain). Finally, Support Vector Machine (SVM) was used to predict the quality of the wines from the measurements made in musts.

\section{Results and discussion}

With the aim to obtain a bioET dedicated to the analysis of must and wines, biosensors specialized in the detection of phenols and sugars (two of the main indicators of the quality of grapes and wines) were developed. The array of sensors was used to analyze grape juices (musts) prepared from different varieties of grapes and the corresponding wines elaborated from those musts.

Voltammograms were characterized by broad peaks whose intensities and positions were determined by the nature of the sensor (enzyme, modifier and substrate) and of the type of sample. The crossselectivity of the sensors included in the array is illustrated in Fig. 1. It is important to remark that the first cycle was always different from the rest, but after 5 cycles, the signals were stabilized and a decrease lower than a $5 \%$ in 15 consecutive cycles was observed. However, once the electrodes were withdrawn from the must/wine solution, proteins, sugars and other wine components were adhered to the sensor surface and sensors could not be reused.

As shown in Fig. 1a, the enzymatic activity of tyrosinase and glucose oxidase induces an important degree of selectivity in the sensor responses. In particular, in the intensity and position of cathodic wave that reflects the consumption of $\mathrm{O}_{2}$ to produce $\mathrm{H}_{2} \mathrm{O}_{2}$ at $-0.6 \mathrm{~V}$ (in the case of GOx) (Jugović et al., 2016) or the reduction of the oxidized quinoid species to the phenolic form at $-0.8 \mathrm{~V}$ in the case of Tyr
(Kirsanov, Mednova, Vietoris, Kilmartin, \& Legin, 2012). Fig. 1b illustrates the remarkable increase in intensity observed when the composite Ppy/AuNPs was used as electron mediator demonstrating the existence of synergistic effects when two electrocatalytic materials are mixed. Finally, in Fig. 1c, the influence of nature of the substrate is illustrated. As observed in the figure, platinum facilitates the electron transfer causing an increase in the intensity of the electrochemical response.

Fig. 1d and e illustrate the responses obtained from wines and musts. The electrochemical responses of musts were characterized by a broad anodic peak at ca. $0.2-0.5 \mathrm{~V}$. The response of wines showed redox pair at negative potentials with a large separation between the anodic and cathodic waves. The dissimilar responses in wines and musts are due not only to their different chemical nature but also to their different viscosity and conductivity.

The positions of the peaks changed from one must to another (or one wine to another), depending on the variety of grape Voltammograms thus reflect the different composition of the samples, mainly in concentration of redox components such as polyphenols and also differences in ionic composition (including protons) which affect the interchange of ions across the polymer/solution interface during oxidation/reduction process. The variety of responses obtained, allowed us to obtain an array of biosensors with a high degree of crossselectivity.

\subsection{Chemometric analysis}

The outputs of the array of sensors were preprocessed to obtain 10 variables per sensor (Gutiérrez-Osuna \& Nagle, 1999). These variables were used as the input for statistical analysis.

\subsubsection{Discrimination capability of the array: PCA and PARAFAC}

Principal Component Analysis (PCA) was used to evaluate the capability of the bioET to discriminate wines and musts according to the variety of grape. PCA scores plots for wines (Fig. 2a) showed welldefined and separated clusters for each variety of grape. Clusters were distributed in the plot according to the alcoholic degree and TPI (Table 1). Thus, Rufete (8) wine (with the lowest values of alcoholic degree and TPI) was clearly separated from the rest of wines. Garnach (2) and Juan García (3) varieties with the same values of alcoholic
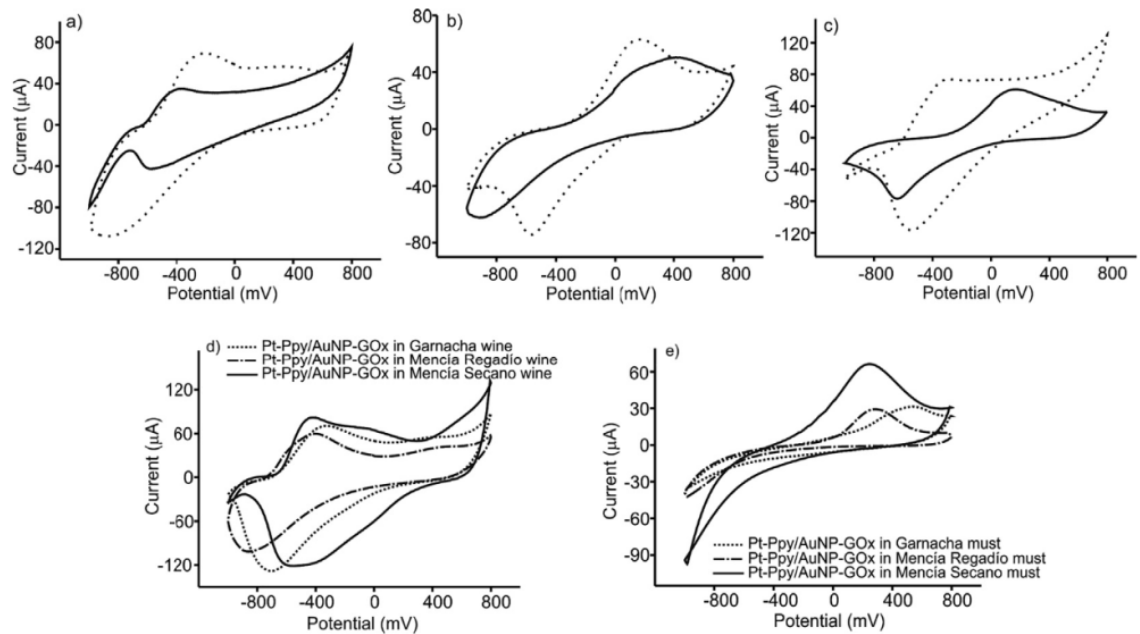

Fig. 1. (a) Pt-Ppy/AuNP-Tyr (dotted line) and Pt-Ppy/AuNP-GOx (solid line) immersed in Rufete wine; (b) SS-Ppy/AuNP-GOx (dotted line) and SS-Ppy-GOx (solid line) immersed in Rufete wine and (c) Pt-Ppy/AuNP-GOx (dotted line) and SS-Ppy/AuNP-GOx (solid line) immersed in Cabernet wine. (d) Voltammetric responses obtained in wines of different varieties of grapes and (e) Voltammetric responses obtained in musts of different varieties of grapes. 

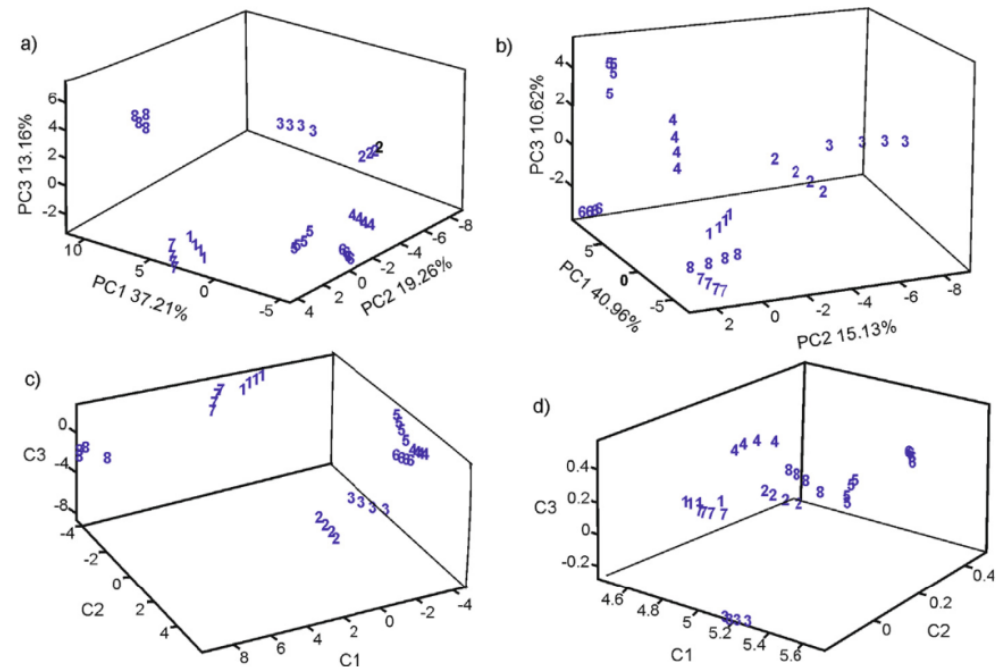

Fig. 2. (a) 3D PCA score plots of the bioET obtained from voltammetric responses in wines; (b) 3D PCA score plots of the bioET obtained from voltammetric responses in musts; (c) 3D PARAFAC score plots of the bioelectronic tongue obtained from voltammetric responses in wines and (d) 3D PARAFAC score plots of the bioelectronic tongue obtained from voltammetric responses in musts. 1 : Cabernet, 2: Garnacha, 3: Juan García, 4: Mencía Regadío, 5: Mencía Secano, 6: Prieto Picudo, 7: Tempranillo and 8: Rufete. degree and TPI were in the same region. Cabernet (1) and Tempranillo (7) varieties with the highest values in TPI and alcoholic degree also appear closer and separated from the rest. The captured information was $69.64 \%$ ( $\mathrm{PC} 1=37.21 \%$; $\mathrm{PC} 2=19.26 \%$; $\mathrm{PC} 3=13.16 \%$ ).

In the case of musts (Fig. 2b), first three principal components explained the $67.2 \%$ of the information $(\mathrm{PC} 1=40.96 \%$; PC2 $=15.13 \%$; PC3 $=10.62 \%$ ). The position of the clusters could not be unequivocally related to the ${ }^{\circ}$ Brix and/or TPI. However, interesting similarities between the relative positions observed in musts with the positions of the clusters obtained in wines were observed. For instance, also in musts, Cabernet (1) and Tempranillo (7) appear close together. Mencía Regadío (4), Mencía Secano (5) and Prieto Picudo (6) were also located nearby. Garnacha (2) and Juan Garcia (3) also appeared together.

This similarity in the results obtained in wines and in musts used to prepare the wines could indicate that the bioET could be used to establish correlations between the quality of grapes and the wines produced form them.

Given the three-way nature of the data (samples $\times$ kernels $\times$ sensors), the information contained in the voltammetric responses was extracted by multi-way modelling using PARAFAC (Kirsanov et al., 2012). Using the core consistency diagnostic tool and the corresponding residual sum of squares (Bro \& Kiers, 2003) three components were able to describe our PARAFAC model. Fig. 2c and 2d show the PARAFAC score scatter plot on three factors, obtained for wines and musts respectively. In the case of PARAFAC the error, in terms of root-mean-square error (RMSE), is the parameter used to confirm the model. In our case low RMSEs were found when performing PARAFAC: 0.687 in the case of wines and 0.089 in the case of musts.

PARAFAC results corroborate the outputs obtained with the twoway PCA. For instance, scatter plot for wines (Fig. 2c) showed that samples were grouped according to their TPI and alcoholic degree. In the case of musts (Fig. 2d) the PARAFAC model also corroborated the results obtained with PCA, but some explicit differences could be observed because in this case, discrimination was clearly linked to the ${ }^{\circ}$ Brix. For example, Cabernet (1) and Tempranillo (7) musts with the same TPI and ${ }^{\circ}$ Brix values appear so close that seem to be overlapped, whereas the must Juan Garcia (3) that shows the same TPI but different ${ }^{\circ}$ Brix appeared separated. Similarly, Garnacha (2) and Rufete (8) samples with the same ${ }^{\circ}$ Brix but a very different TPI, appeared close one from each other. Finally, Juan Garcia with the lowest ${ }^{\circ}$ Brix was located quite separated from the rest.
PARAFAC analysis was also used to obtain information about the data structure by means of Mode plots. Mode plots display in a simple and interpretable manner the relevant information and the sources of data variability. Given the three-way nature of the bioET data (samples $\times$ kernels $\times$ sensors) three Mode plots are analyzed: Mode 1 gives information about the contribution of the samples to the three components, Mode 2 about the contribution of kernels and Mode 3 about the contribution of the sensors and the cross-selectivity. Fig. 3 corresponds to Mode plots obtained for wines (upper figures) and musts (lower figures) samples and lines black, grey and dotted refer to first, second and third component, respectively.

Mode 1 plots (Fig. 3a and d) indicate that the first Principal Component (black line) is bringing the most relevant information. The in formation contained in the second (grey line) and third (dotted line) components is relatively small. Mode 2 shows the contribution of the 10 kernels (the 10 sections in which voltammograms are divided after applying Kernel functions). A difference between wines and musts can be observed (Fig. 3b and e). In the case of wines (Fig. 3b) kernels 3-10, are the most important contributors to the first principal Component. This means that almost the whole voltammogram (except kernels 1 and 2 , corresponding to the left part of the voltammograms) contribute to the capability of discrimination of the bioET. This is in good agreement with the shapes of voltammograms shown in Fig. 1 for wines, were it can be observed that positions and shapes of the peaks depend on the type of wine and the variety of grape. In the case of musts, Mode 2 (Fig. 3e), kernels 5 to 10 bring more information than kernels 1-4. Again, this can be easily explained taking into account that at positive potentials, the oxidation of many components of musts such as phenols takes place. Finally, Mode 3 (Fig. 3c and f) demonstrates that all sensors contribute equally to the first principal component, and confirms the important degree of cross-selectivity of the sensors forming the array.

3.1.2. Correlations of the chemical composition: Partial Least Squares and Support Vector Machines regression

As it has been stated before, ETs give an overall view of the samples, however, the signals provided by ETs, can be correlated with the chemical composition of the samples using regression techniques. Table 2 collects the statistical parameters obtained from the Partial Least Squares PLS-1 regression models correlating the output of the bioET with chemical analytical parameters measured in musts and wines. Models were validated by means of the "leave-one-out" method. Good 

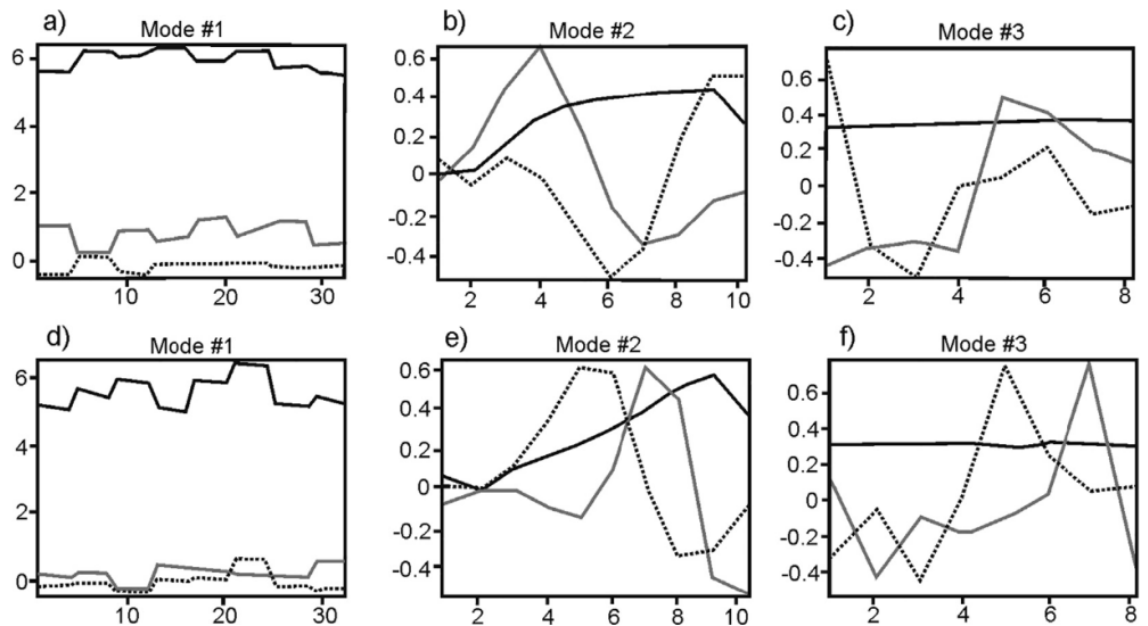

Fig. 3. Mode plots obtained from PARAFAC analysis in wines (a-c) and musts (d-f). Mode \#1: Contribution of the samples, Mode \#2: Contribution of the 10 kernels, Mode \#3: Contribution of the sensors. Red: Component 1, Blue: Component 2 and Yellow: Component 3.

Table 2

Statistical parameters obtained for the PLS-1 regression models established between the chemical parameters and the bioET.

Must

\begin{tabular}{llllll}
\hline Parameters & $\mathrm{R}_{\mathrm{C}}^{2 \mathrm{a}}$ & $\mathrm{RMSE}_{\mathrm{C}}{ }^{\mathrm{b}}$ & $\mathrm{R}_{\mathrm{P}}^{2 \mathrm{C}}$ & $\mathrm{RMSE}_{\mathrm{P}}{ }^{\mathrm{d}}$ & Number of components \\
\hline TPI & 0.8751 & 1.5379 & 0.7687 & 2.1602 & 3 \\
Brix & 0.9390 & 0.2845 & 0.8455 & 0.4675 & 3 \\
Wines & & & & & \\
\hline Parameters & $\mathrm{R}_{\mathrm{C}}^{2 \mathrm{a}}$ & $\mathrm{RMSE}_{\mathrm{C}}{ }^{\mathrm{b}}$ & $\mathrm{R}_{\mathrm{P}}{ }^{2 \mathrm{c}}$ & $\mathrm{RMSE}_{\mathrm{P}}{ }^{\mathrm{d}}$ & Number of components \\
\hline TPI & 0.9442 & 1.5192 & 0.8874 & 2.2273 & 3 \\
Alcoholic degree & 0.9268 & 0.2103 & 0.8615 & 0.2985 & 3 \\
\hline
\end{tabular}

${ }^{a}$ Squared correlation coefficient in calibration.

b Root mean square error of calibration.

Squared correlation coefficient in prediction.

${ }^{\mathrm{d}}$ Root mean square error of prediction.

correlations (in both calibration and prediction) were found for the TPI, the Brix degree, which are chemical parameters usually used to evaluate the quality of grapes. Similarly, correlations were found with the alcoholic degree and the TPI which are usual markers of the quality of wines. The good correlations can be attributed to the working principle of our bioET that shows specificity towards phenols (improved by the presence of tyrosinase) and sugars (improved by the presence of GOx). The good correlation with the alcoholic degree of a wine can be due to the fact that the alcoholic degree is directly related with the sugar content of the grapes used to elaborate them.

\subsubsection{Support Vector Machine (SVM)}

It is well known that the characteristics and quality of a wine depend in a large extent on the characteristics and quality of the grapes used in the elaboration. In this work, the bioET was used to predict chemical characteristics of wines from voltammograms collected in grapes. For this purpose, Support Vector Machine Regression (SVMR) was used to predict the total polyphenol index (TPI) and the alcoholic degree of wines from data measured using the bioET in grapes. Two data matrices were developed: the " $\mathrm{X}$ " matrix (predictors) was constructed using the data from musts registered using the bioET and a "Y" matrix (responses) contained data of chemical parameters (TPI or alcoholic degree) of wines. The SVMR models were created using SVM type: Regression (epsilon SVR), kernel type: Linear, C value: 1, weights: All 1.0 and cross validation segments size: 10 , in all cases. Prediction values of TPI and alcoholic degree obtained after creating the regression models were close to those obtained by means of traditional methods. SVMR models showed coefficients of correlation of 0.9899 in the calibration and 0.9469 in the prediction in the case of TPI, with errors of 0.7095 in the calibration and 1.6353 in the prediction. In the case of the alcoholic degree, the coefficients of correlation were 0.9910 in the calibration and 0.9536 in the prediction with errors of 0.0836 in the calibration and 0.1833 in the prediction.

After the SVMR model was constructed, the regression models were used to predict the TPI and the alcoholic degree of wines samples that were not included in the creation of the model. Results are shown in Table 3 versus the experimental results obtained by traditional

Table 3

TPI and alcoholic degree predicted in wine samples after applying the regression models previously performed by SVM.

\begin{tabular}{|c|c|c|c|c|c|c|}
\hline Sample & $\begin{array}{l}\text { TPI predicted in wine by } \\
\text { means of SVMR }\end{array}$ & $\begin{array}{l}\text { TPI obtained by } \\
\text { traditional methods }\end{array}$ & $\begin{array}{l}\mid \text { Relative error } \mid \\
\%\end{array}$ & $\begin{array}{l}\text { Alcoholic degree predicted in } \\
\text { wine by means of SVMR }\end{array}$ & $\begin{array}{l}\text { Alcoholic degree obtained by } \\
\text { traditional methods }\end{array}$ & $\begin{array}{l}\mid \text { Relative error } \mid \\
\%\end{array}$ \\
\hline Cabernet & 56.9 & 57 & 0.2 & 13.00 & 13.03 & 0.2 \\
\hline Garnacha & 43.7 & 41 & 6.6 & 11.69 & 11.36 & 2.9 \\
\hline Juan García & 40.3 & 41 & 1.7 & 11.39 & 11.40 & 0.1 \\
\hline Mencía Regadío & 44.1 & 44 & 0.2 & 12.04 & 12.01 & 0.2 \\
\hline Mencía Secano & 53.1 & 54 & 1.7 & 12.66 & 12.81 & 1.2 \\
\hline Prieto Picudo & 46.2 & 46 & 0.4 & 12.52 & 12.48 & 0.3 \\
\hline Rufete & 41.5 & 39 & 6.4 & 11.42 & 11.13 & 2.6 \\
\hline Tempranillo & 52.8 & 53 & 0.4 & 13.24 & 13.28 & 0.3 \\
\hline
\end{tabular}


chemical methods.

\section{Conclusions}

A bioET combining biosensors sensitive to phenols and sugars has been developed. The use of Ppy/AuNPs as the electron mediator in creases the sensitivity and cross-selectivity of the sensors, improving in this way the performance of the array of biosensors. The results revealed that using PCA and PARAFAC it was possible to discriminate samples according to the variety of grape. PLS-1 regressions showed good correlations with the chemical parameters. Using SVMR it has been possible to predict the TPI and the alcoholic degree of wines, from data provided by the bioET in the corresponding grapes. This result is of great interest for the food industry and has advantages with respect to already existing methods because it opens the possibility to predict some of the characteristics of the final wine, from the beginning of the process and in a single measurement.

\section{Acknowledgements}

Financial support by MINECO-FEDER (AGL2015-67482-R) and the Junta de Castilla y Leon FEDER (VA275P18) is gratefully acknowledged. Celia Garcia-Hernandez would also like to thank Junta de Castilla y León for a grant (BOCYL-D-4112015-9).

\section{Conflict of interest}

The authors declare that they have no conflicts of interest in the research.

\section{Appendix A. Supplementary data}

Supplementary data to this article can be found online at https:// doi.org/10.1016/j.foodchem.2019.03.107

\section{References}

Apetrei, I. M., Rodríguez-Méndez, M. L., Apetrei, C., Nevares, L., del Alamo, M., \& de Saja, J. A. (2012). Monitoring of evolution during red wine aging in oak barrels and al-
ternative method by means of an electronic panel test. Food Research International, 45 , 244-249. https://doi.org/10.1016/j.foodres.2011.10.034

Apetrei, I. M., \& Apetrei, C. (2013). Amperometric biosensor based on polypyrrole and tyrosinase for the detection of tyramine in food samples. Sensors and Actuators B: Chemical, 178, 40-46. https://doi.org/10.1016/j.snb.2012.12.064.

Baldeón, E. O., Alcañiz, M., Masot, R., Fuentes, E. M., Barat, J. M., \& Grau, R. (2015). Voltammetry pulse array developed to determine the antioxidant activity of cam camu (Myrciaria dubia (H.B.K.) McVaug) and tumbo (Passiflora mollisima (Kunth) L.H. Bailey) juices employing voltammetric electronic tongues. Food Control, 54, 181-187. https://doi.org/10.1016/j.foodcont.2015.01.044

Bro, R., \& Kiers, H. A. L. (2003). A new efficient method for determining the number of components in PARAFAC models. Journal of Chemometrics, 17, 274-286. https://doi. org $10.1002 / \mathrm{cem} .801$

Buratti, S., Ballabio, D., Giovanelli, G., Dominguez, C. M. Z., Moles, A., Benedetti, S., \& Sinelli, N. (2011). Monitoring of alcoholic fermentation using near infrared and mid infrared spectroscopies combined with electronic nose and electronic tongue.

Analytica Chimica Acta, 697, 67-74. https://doi.org/10.1016/j.aca.2011.04.020.

, X., Capdevila, J., Mínguez, S., \& del Valle, M. (2014). Voltammetric BioElectronic Tongue for the analysis of phenolic compounds in rosé cava wines. Food Resec

Cetó, X., González-Calabuig, A., Crespo, N., Pérez, S., Capdevila, J., Puig-Pujol, A., \& de Valle, M. (2017). Electronic tongues to assess wine sensory descriptors. Talanta, 162, Valle, M. (2017). Electronic tongues to assess wine sensory

Friedel, M., Patz, C.-D., \& Dietrich, H. (2013). Comparison of different measurement techniques and variable selection methods for FT-MIR in wine analysis. Food Chemistry, 141, 4200-4207. https://doi.org/10.1016/j.foodchem.2013.06.120.

García-Hernández, C., García-Cabezón, C., Medina-Plaza, C., Martín-Pedrosa, F., Blanco, Y., de Saja, J. A., \& Rodríguez-Méndez, M. L. (2015). Electrochemical behavior of polypyrrol/AuNP composites deposited by different electrochemical methods: Sensing properties towards catechol. Beilstein Journal of Nanotechnology, 6 , 2052-2061. https://doi.org/10.3762/bjnano .6.209.

García-Hernández, C, García-Cabezón, C. Martin-Pedrosa, F., De Saja, J. A., \& Rodríguez-Méndez, M. L. (2016), Layered composites of PEDOT/PSS/nanoparticles and PEDOT/PSS/phthatocyanines as electron mediators for sensors and biosensors. Beilstein Journal of Nanotechnology, 7, 1948-1959.
Ghasemi-Varnamkhasti, M., Rodríguez-Méndez, M. L., Mohtasebi, S. S., Apetrei, C Lozano, J., Ahmadi, H.,... Antonio de Saja, J. (2012). Monitoring the aging of beer using a bioelectronic tongue. Food Control, 25, 216-224. https://doi.org/10.1016/j. using a bioelectronic

Gil-Sánchez, L., Soto, J., Martínez-Máñez, R., Garcia-Breijo, E., Ibáñez, J., \& Llobet, E (2011). A novel humid electronic nose combined with an electronic tongue for as (2011). A novel humid electronic nose combined with an electronic tongue for
sessing deterioration of wine. Sensors and Actuators A: Physical, 171, 152-158. https://doi.org/10.1016/j.sna.2011.08.006.

Giménez-Gómez, P., Escudé-Pujol, R., Capdevila, F., Puig-Pujol, A., Jiménez-Jorquera, C., \& Gutiérrez-Capitán, M. (2016). Portable electronic tongue based on microsensors for the

Gutiérrez-Capitañ, M., Capdevila, F., Vila-Planas, J., Domingo, C., Bütgenbach, S., quality control of wines. Journal of Sensors, 2014, 1-10. https://doi. org /10.1155/ 2014/598317.

Gutiérrez-Osuna, R., \& Nagle, H. T. (1999). A method for evaluating data-preprocessing techniques for odour classification with an array of gas sensors. IEEE Transactions on Systems, Man and Cybernetics, Part B, 29, 626-632. https://doi.org/10.1109/3477. 790446 .

Hayasaka, Y., Black, C. A., Hack, J., \& Smith, P. (2017). Structural characterization of reaction products of caftaric acid and bisulfite present in a commercial wine using
high resolution mass spectrometric and nuclear magnetic resonance techniques. Food Chemistry, 230, 99-107. https://doi.org/10.1016/j.foodchem.2017.03.005.

International Organisation of Yine and Wine (2011). Compendium of international methods of wine and must analysis. International Organisation of Vine and Wine https:// doi.org/ISBN Volume I: 979-10-91799-65-2

Jugović, B., Grgur, B., Antov, M., Knežević-Jugović, Z., Stevanović, J., \& Gvozdenović, M. (2016). Polypyrrole-based enzyme electrode with immobilized glucose oxidase for electrochemical determination of glucose. International Journal of Electrochemical Science, 11, 1152-1161

Kirsanov, D., Mednova, O., Vietoris, V., Kilmartin, P. A., \& Legin, A. (2012). Towards reliable estimation of an "electronic tongue" predictive ability from PLS regressio models in wine analysis. Talanta, 90, 109-116. https://doi.org/10.1016/j.talanta. 2012.01.010.

Lvova, L., Guanais Goncalves, C., Petropoulos, K., Micheli, L., Volpe, G., Kirsanov, D., .. Paolesse, R. (2016). Electronic tongue for microcystin screening in waters. Biosen sers and Bioelectronics, 80, 154-160. https://doi.org/10.1016/j.bios.2016.01.050.

Mavrikou, S., Flampouri, E., Iconomou, D., \& Kintzios, S. (2017). Development of a cellular biosensor for the detection of aflatoxin B1, based on the interaction of membrane engineered Vero cells with anti-AFB1 antibodies on the surface of gold nanoparticle screen printed electrodes. Food Control, 73, 64-70. https://doi.org/10.1016, j.foodcont.2016.06.002.

Medina-Plaza, C., de Saja, J. A., Fernández-Escudero, J. A., Barajas, E., Medrano, G., \& Rodríguez-Méndez, M. L. (2016). Array of biosensors for discrimination of grapes according to grape variety, vintage and ripeness. Analytica Chimica Acta, 947, 16-22. hitps./2

Medina-Plaza, C., Garcia-Hernăndez, C., de Saja, J. A., Fernández-Escudero, J. A., Barajas, E., Medrano, G., ... Rodríguez-Mendez, M. L. (2015). The advantages of disposable Food Science and Technolog, 62, 940-947. hos 027.

Musingarabwi, D. M., Nieuwoudt, H. H., Young, P. R., Eyéghè-Bickong, H. A., \& Vivier, M. A. (2015). A rapid qualitative and quantitative evaluation of grape berries at various A. (2015). A rapid qualitative and quatritive infrared spectroscopy and multivariate data analysis. Food Chemistry, 190, 253-262. https://doi.org/10.1016/J. foodchem.2015.05.080.

Peris, M. \& Escuder-Gilabert, L. (2016). Electronic noses and tongues to assess food authenticity and adulteration. Trends in Food Science \& Technology, 58, 40-54. authenticity and adulteration. Trends in F14d
https://doi.org/10.1016/itifs.2016.10.014.

Pigani, L., Culetu, A., Ulrici, A., Foca, G., Vignali, M., \& Seeber, R. (2011). Pedot modified electrodes in amperometric sensing for analysis of red wines samples. Food Chemistry, 129, 226-233. https://doi.org/10.1016/j.foodchem.2011.04.046.

Prieto, N., Gay, M., Vidal, S., Aagaard, O., de Saja, J. A., \& Rodríguez-Méndez, M. L. (2011). Analysis of the influence of the type of closure in the organoleptic characteristics of a red wine by using an electronic panel. Food Chemistry, 129, 589-594. https://doi.org/10.1016/j.foodchem.2011.04.071.

Rodríguez-Méndez, M. L. (2016). Electronic noses and tongues in food science. Academic Press.

Rodríguez-Méndez, M. L., Apetrei, C., Gay, M., Medina-Plaza, C., de Saja, J. A., Vidal, S., Wirth, J., \& Cheynier, V. (2014). Evaluation of oxygen exposure levels and polyphenolic content of red wines using an electronic panel formed by an electronic nose and an electronic tongue. Food Chemistry, 155C, 91-97. https://doi.org/10.1016/j. foodchem.2014.01.021.

Rudnitskaya, A., Rocha, S. M., Legin, A., Pereira, V., \& Marques, J. C. (2010). Evaluation of the feasibility of the electronic tongue as a rapid analytical tool for wine age prediction and quantification of the organic acids and phenolic compounds. The casestudy of Madeira wine. Analytica Chimica Acta, 662, 82-89. https://doi.org/10.1016/ j.aca.2009.12.042

Smyth, H., \& Cozzolino, D. (2013). Instrumental methods (spectroscopy, electronic nose, and tongue) as tools to predict taste and aroma in beverages: Advantages and limitations. Chemical Reviews, 113, 1429-1440. https://doi.org/10.1021/cr300076c. Toko, K. (2013). Biochemical sensors: Mimicking gustatory and olfactory senses. Pan Stanford Publishing.

Zeravik, J., Hlavacek, A, Lacina, K. \& Skládal, P. (2009). State of the art in the field of electronic and bioelectronic tongues - Towards the analysis of wines. Electroanalysis, 21, 2509-2520. https://doi.org/10.1002/elan.200900285. 


\subsection{Paper VI}

Layered composites of PEDOT/PSS/nanoparticles and PEDOT/PSS/phthalocyanines as electron mediators for sensors and biosensors.

By Celia García-Hernández, Cristina García-Cabezón, Fernando MartínPedrosa, José Antonio de Saja and María Luz Rodríguez-Méndez.

Beilstein Journal of Nanotechnology 7 (2016) 1948-1959.

(doi: 10.3762/bjnano.7.186) 


\subsubsection{Motivation and objective}

The food industry requires the development of new sensors with improved performance in terms of sensitivity, detection limit and selectivity. In the last few years, the conducting polymer PEDOT:PSS (poly(3,4-ethylenedioxythiophene:poly(styrenesulfonate)) has received much attention for manufacturing flexible or rigid structures of nanocomposites with improved characteristics for sensing applications. This polymer consists of a mixture of two ionomers, poly(poly(3,4-ethylenedioxythiophene), which is a conjugated polymer carrying a positive charge, and sodium polystyrene sulfonate, where some sulfonyl groups are deprotonated and carry a negative charge. Both together, they form a macromolecular salt, PEDOT:PSS, with such properties as high electrical conductivity, biocompatibility, mechanical elasticity and high transparency, along with the reversible charge/discharge ability from the doping/de-doping of the polymer chain. These characteristics make this polymer very attractive for developing new electrochemical sensors over other organic conducting polymers.

However, the applications of organic semiconductor PEDOT:PSS film-based sensors might be limited in aqueous solutions due to the highly hydrophilic property caused by the presence of PSS, which acts as a dopant to stabilize the PEDOT:PSS to avoid the poor solubility and processability of PEDOT. This fact gives the conducting polymer a degree of water solubility which leads to the film breaking on the electrode surface and, therefore, reducing the electrical conductivity and electrochemical performance. To solve this limitation and make the polymer less hydrophilic, researchers have combined PEDOT:PSS with other sensing materials, such as graphene or carbon nanotubes, and they have added a binder to strengthen the adhesion of the parts. Gold nanoparticles and metalphthalocyanines are classical electrocatalytic materials that might be good candidates to form composites with PEDOT:PSS in electrochemical sensors for applications in the food industry. In addition, the strong electrochemical properties of the asfabricated sensors can be used to make biosensors in which the PEDOT:PSS composites will act as mediators to shuttle the electrons between the enzyme and the electrode. Thanks to its high conductivity, stability, organic nature and good compatibility with such biomolecules as enzymes, PEDOT is one of the most suitable materials for bioelectronics among the conducting polymers.

The objective of this work is to design new electrodes based on composites of PEDOT:PSS with a secondary electrocatalytic material, such as gold nanoparticles (AuNPs), lutetium bisphthalocyanine $\left(\mathrm{LuPC}_{2}\right)$ and copper phthalocynine (CuPc), to enhance the 
electrocatalytic activity for two diphenols, such as catechol and its isomeric form hydroquinone. Polyphenol compounds with the catechol group are expected to represent the main polyphenol type in red wines. Furthermore, the enzymes tyrosinase and laccase have been implemented in the mentioned composite-based sensors to improve their electrochemical performance. Tyrosinase is selective to the oxidation of orto-diphenols, whereas laccase catalyzes the oxidation of a larger variety of polyphenols.

\subsubsection{Summary of the research work}

PEDOT:PSS-based sensors were made using the spin-coating technique. The conducting polymer was first deposited onto an ITO surface and then spin-coated. After the deposition, the electrodes were annealed to improve adhesion onto the ITO surface. Then, the secondary electrocatalytic material (EM) (e.g., copper phthalocyanince, lutetium phthalocyanine or gold nanoparticles) was deposited onto the previously modified ITO surface and spin-coated with the same experimental conditions as before to obtain PEDOT:PSS/CUPC, PEDOT:PSS/LUPC2 and PEDOT:PSS/AuNP sensors. AuNPs were previously synthesized following the Turkevich method.

Enzymatic biosensors were prepared using tyrosinase (Tyr) for catechol detection and laccase (Lac) for hydroquinone detection. For this purpose, the enzyme solution was drop-casted onto the modified ITO surface and dried at room temperature to obtain PEDOT:PSS/EM-Tyr and PEDOT:PSS/EM-Lac biosensors. Glutaraldehyde was used as the cross-linker during enzymes deposition.

The surface morphology of the PEDOT:PSS sensors was studied by SEM, evidencing a regular, smooth and homogeneous structure for PEDOT:PSS, PEDOT:PSS/CuPC and PEDOT:PSS/ LUPC2 films. In the PEDOT:PSS/AuNP films, SEM images showed AuNPs located in clusters with an average size of $40-60 \mathrm{~nm}$.

Conductivity measurements (resistance and resistivity) demonstrated that PEDOT:PSS/EM presented a higher conductivity than the non-modified PEDOT:PSS sensor following the sequence: PEDOT:PSS/LuPC 2 > PEDOT:PSS/AuNPs > PEDOT:PSS/CuPc. This result implies that the electron transfer will be improved with the layered composites. This fact 
was later corroborated by the electrochemical impedance spectroscopy (EIS), where it was observed that the value of the electron-transfer resistance in PEDOT:PSS/EM sensors was about half that observed in the PEDOT:PSS sensor.

The electrochemical behavior of PEDOT:PSS-based sensors for di-phenols was analyzed by means of cyclic voltammetry. Voltammograms registered in catechol showed a shift of the cathodic peak to lower potentials when using PEDOT:PSS/EM. In the case of PEDOT:PSS/LuPc2, the electrocatalytic effect was stronger than for the rest, showing more reversible peaks and higher intensities. Although the response of the sensors for hydroquinone did not show a decrease in the cathodic peak to lower potentials, the intensities did increase on both redox peaks.

Biosensors PEDOT:PSS/EM-Tyr and PEDOT:PSS/EM-Lac showed higher intensities in the cathodic wave than non-biosensors when analyzing catechol and hydroquinone. However, the oxidation peaks were not observed. This result evidences the electron mediator performance of the PEDOT:PSS/EM composites.

The limits of detection obtained with the biosensors were in the range of $10^{-6}-10^{-7} \mathrm{~mol} \cdot \mathrm{L}^{-1}$, one order of magnitude lower than those obtained with non-biosensors $10^{-5}-10^{-6} \mathrm{~mol} \cdot \mathrm{L}^{-1}$.

The dynamic behavior of sensors for catechol and hydroquinone was studied by varying the scan rate and registering the corresponding voltammograms. Results showed that the cathodic peak potentials shifted to more negative values when the scan rate was increased, evidencing the irreversibility of the redox process. Moreover, the cathodic peak currents varied linearly with the square root of the scan rate, corroborating the diffusion-controlled nature of the electrode reaction. The slope values calculated in the PEDOT:PSS biosensors were higher than those observed in non-biosensors, so this result confirmed the improvement in the charge transfer due to the enzymatic activity.

Finally, the repeatability and reproducibility of the as-prepared sensors and biosensors was studied, resulting in coefficients of variation lower than $5 \%$ in both tests. 


\title{
Layered composites of PEDOT/PSS/nanoparticles and PEDOT/PSS/phthalocyanines as electron mediators for sensors and biosensors
}

\author{
Celia García-Hernández, Cristina García-Cabezón, Fernando Martín-Pedrosa, \\ José Antonio De Saja and María Luz Rodríguez-Méndez ${ }^{*}$
}

\section{Full Research Paper}

\section{Address:}

Grupo Uvasens, Escuela de Ingenierías Industriales, Universidad de Valladolid, Paseo del Cauce 59, 47011 Valladolid, Spain

Email:

Maria Luz Rodriguez-Méndez' - mluz@eii.uva.es

' Corresponding author

Keywords:

catechol; gold nanoparticle hydroquinone; PEDOT/PSS:

phthalocyanine; voltammetric sensor
Beilstein J. Nanotechnol. 2016, 7, 1948-1959.

doi:10.3762/bjnano.7.186

Received: 30 August 2016

Accepted: 16 November 2016

Published: 08 December 2016

This article is part of the Thematic Series "Functional materials for environmental sensors and energy systems".

Guest Editor: M. Penze

2016 Garcia-Hernández et al.; licensee Beilstein-Institut. License and terms: see end of document.

\begin{abstract}
The sensing properties of electrodes chemically modified with PEDOT/PSS towards catechol and hydroquinone sensing have been successfully improved by combining layers of PEDOT/PSS with layers of a secondary electrocatalytic material such as gold nanoparticles (PEDOT/PSS/AuNPs), copper phthalocyanine (PEDOT/PSS/CuPc) or lutetium bisphthalocyanine ( $\mathrm{PEDOT} / \mathrm{PSS} / \mathrm{LuPc}_{2}$ ). Layered composites exhibit synergistic effects that strongly enhance the electrocatalytic activity as indicated by the increase in intensity and the shift of the redox peaks to lower potentials. A remarkable improvement has been achieved using PEDOT/PSS/ $\mathrm{LuPc}_{2}$, which exhibits excellent electrocatalytic activity towards the oxidation of catechol. The kinetic studies demonstrated diffusion-controlled processes at the electrode surfaces. The kinetic parameters such as Tafel slopes and charge transfer coefficient $(\alpha)$ confirm the improved electrocatalytic activity of the layered electron mediators. The peak currents increased linearly with concentration of catechol and hydroquinone over the range of $1.5 \times 10^{-4}$ to $4.0 \times 10^{-6} \mathrm{~mol} \cdot \mathrm{L}^{-1}$ with a limit of detection on the scale of $\mu \mathrm{mol} \cdot \mathrm{L}^{-1}$. The layered composite hybrid systems were also found to be excellent electron mediators in biosensors containing tyrosinase and laccase, and they combine the recognition and biocatalytic properties of biomolecules with the unique catalytic features of composite materials. The observed increase in the intensity of the responses allowed detection limits of $1 \times 10^{-7} \mathrm{~mol} \cdot \mathrm{L}^{-1}$ to be attained.
\end{abstract}




\section{Introduction}

The assessment of phenols has been successfully achieved using electrodes chemically modified with a variety of materials [1-10]. In addition, some mixtures of electrocatalytic materials such as polyaniline or polypyrrole with graphene can enhance the sensitivity of the sensors while lowering the detection limits [11-13]

Poly(3,4-ethylene dioxythiophene)/poly(styrene sulfonic acid) (PEDOT/PSS) is an appealing electrocatalytic material due to its high conductivity and low redox potential. PEDOT/PSS is soluble in water and the polymer can be mixed with watersoluble electrocatalytic materials or colloidal metal nanoparticles providing an excellent method to modulate the sensing properties by means of a synergistic effect $[14,15]$. However, a large number of electrocatalytic materials are soluble in organic solvents and this fact limits the number of electrocatalytic materials that can be used to modulate the PEDOT/PSS properties. One possible strategy to avoid this problem is to develop layered composites where a film of a secondary electrocatalytic material is deposited on top of a PEDOT/PSS layer

The first objective of this work was to develop novel electrochemical sensors based on layered composites formed by alternating layers of PEDOT/PSS and a secondary electrocatalytic material (EM) (PEDOT/PSS/EM) and to evaluate the existence of synergistic effects. As EMs, three different materials with different characteristics and electrocatalytic activity towards phenols were tested, including gold nanoparticles (AuNPs), a copper phthalocyanine ( $\mathrm{CuPc}$ ) (a p-type semiconductor) and a lutetium bisphthalocyanine $\left(\mathrm{LuPc}_{2}\right)$ (a sandwich-type derivative with free radical character which is an intrinsic semiconductor) $[9,16-18]$.

On the other hand, enzymatic electrochemical biosensors based on phenol oxidases are a good alternative to analyze phenols due to their high sensitivity and selectivity. Tyrosinase oxidizes monophenols and o-diphenols to the corresponding quinone whereas laccase catalyzes the oxidation of a larger variety of aromatic compounds such as substituted mono- and poly-phenols, with subsequent formation of radicals, which are converted to quinones in the second stage of the oxidation [19]. Tyrosinase (Tyr) and laccase (Lac) must be combined with electron mediators to facilitate the transfer of electrons from the enzyme to the electrode [20]. PEDOT/PSS is becoming popular as an electron mediator in biosensing $[21,22]$. Gold nanoparticles and phthalocyanines have also been positively demonstrated as electron mediators in tyrosinase biosensors [16,23-26]. Also, in the case of biosensors, an improvement of the electron mediator activity could be expected by using layered composites formed by two electron mediators. For this reason, the second objective of this work was to develop phenol biosensors containing Tyr or Lac using layered composites of PEDOT/PSS and AuNPs, CuPc or $\mathrm{LuPc}_{2}$ as electron mediators and to investigate the existence of synergistic effects in the electron transfer occurring during biosensing

In order to pursue the two objectives proposed, PEDOT/PSS/ EM electrodes were prepared by depositing a layer of AuNPs, $\mathrm{CoPc}$ or $\mathrm{LuPc}_{2}$ on the top of the PEDOT/PSS film by means of spin coating to obtain PEDOT/PSS/AuNP, PEDOT/PSS/CuPc

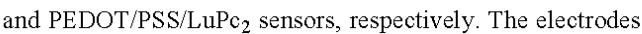
were used as the working electrodes in cyclic voltammetry. The sensing properties and any synergistic effects in the layered composite electrodes were evaluated towards catechol $(1,2-$ dihydroxybenzene) and hydroquinone (1,4-dihydroxybenzene) which are two dihydroxybenzene isomers with many ubiquitous industrial applications $[27,28]$. Additionally, the kinetic parameters and limit of detection were calculated.

Further studies were carried out using the modified electrodes combined with tyrosinase (PEDOT/PSS/EM-Tyr) and laccase (PEDOT/PSS/EM-Lac) enzymes. The electron mediator properties of the layered composites with respect to the performance of biosensors containing tyrosinase or laccase were evaluated.

\section{Results and Discussion Structure and conductivity of the PEDOT/ PSS/EM sensors}

ITO electrodes were modified by depositing a PEDOT/PSS layer by means of spin coating. Layered composite electrodes were prepared by depositing layers of a secondary electrocatalytic material ( $\mathrm{AuNPs}, \mathrm{CoPc}$ or $\mathrm{LuPc}_{2}$ ) on top of the PEDOT/ PSS film to obtain PEDOT/PSS/AuNP, PEDOT/PSS/CuPc and $\mathrm{PEDOT} / \mathrm{PSS} / \mathrm{LuPc}_{2}$, respectively. The surface morphologies of the modified electrodes were analyzed by SEM. As observed in Figure 1, the PEDOT/PSS film showed a regular, smooth and homogeneous structure. Further modification with $\mathrm{CuPc}$ and $\mathrm{LuPc}_{2}$ did not affect the morphology. SEM images of the PEDOT/PSS/AuNP electrode confirmed that small clusters of 40-60 nm diameter AuNPs were homogeneously distributed on the surface of the film. The increase in the effective surface area due to the AuNPs could be highly beneficial for electrochemical applications in sensors.

The square resistance and resistivity were measured with the four-point probe test (Table 1). The current-voltage curves obtained from PEDOT/PSS and PEDOT/PSS/EM electrodes exhibited a good linear fit with correlation coefficients higher than 0.999 . 


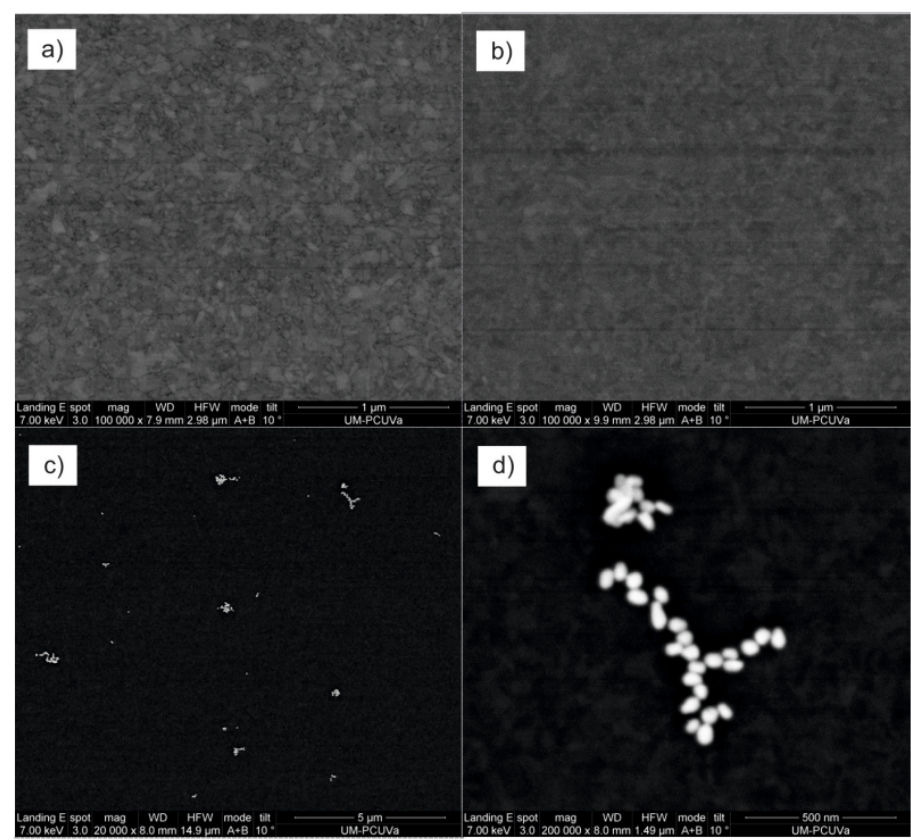

Figure 1: SEM images of electrodes modified with a) PEDOT/PSS, b) PEDOT/PSS/CuPc, c) PEDOT/PSS/AuNP and d) PEDOT/PSS/AuNP (higher magnification).

Table 1: Square resistance $\left(R_{\mathrm{Sq}}\right)$ and conductivity $(\rho)$ of the PEDOT PSS and PEDOT/PSS/EM electrodes.

\begin{tabular}{lll} 
& $\begin{array}{l}R_{\mathrm{Sq}} \\
\left(\Omega \cdot \mathrm{sq}^{-1}\right)\end{array}$ & $\begin{array}{l}\rho \\
\left(\times 10^{-6} \Omega \cdot \mathrm{m}\right)\end{array}$ \\
\hline PEDOT/PSS & 13.9 & 27.9 \\
PEDOT/PSS/LuPc 2 & 6.4 & 15.7 \\
PEDOT/PSS/CuPc & 9.6 & 22.4 \\
PEDOT/PSS/AuNP & 7.9 & 19.7
\end{tabular}

It has been reported that the addition of different dopants to an aqueous solution of PEDOT/PSS produces an enhancement in the conductivity of PEDOT/PSS films obtained from the solution $[29,30]$. In our case, the additives were deposited as a thin film on the top of the PEDOT/PSS layer and the same effect was observed. Layered composites exhibited better conductivity than PEDOT/PSS following the order PEDOT/PSS/LuPc ${ }_{2}$ $>$ PEDOT/PSS/AuNPs $>$ PEDOT/PSS/CuPc. This enhanced conductivity can contribute to the observed improvement of the electron transfer rate of the sensors that will be shown in the following.
The mechanism of the conductivity enhancement can be different depending on the material deposited on the PEDOT/PSS layer [29-31]. In the case of CuPc or AuNPs, the improvement in the conductance can be due to the increase in the charge carrier mobility and/or in the large effective surface provided by the metallic AuNPs. In the case of $\mathrm{LuPc}_{2}$, which is an intrinsic semiconductor and has a free radical character [32], the enhancement can be due to the increase in charge carriers produced by the interaction between the PEDOT/PSS chains and the free carriers in $\mathrm{LuPc}_{2}$.

\section{Electrochemical characterization of the PEDOT/PSS/EM sensors}

The electrochemical characteristics of PEDOT/PSS/EM electrodes were analyzed using cyclic voltammetry. Voltammograms of PEDOT/PSS, PEDOT/PSS/AuNPs, PEDOT/PSS/ $\mathrm{CuPc}$ and $\mathrm{PEDOT} / \mathrm{PSS} / \mathrm{LuPc}_{2}$ electrodes immersed in catechol and hydroquinone $1.5 \times 10^{-4} \mathrm{~mol} \cdot \mathrm{L}^{-1}$ with $0.01 \mathrm{~mol} \cdot \mathrm{L}^{-1}$ phosphate buffer as the supporting electrolyte are shown in Figure 2 The response of ITO glass is not shown in the figure due to the low intensity, characterized by one cathodic peak at $-0.4 \mathrm{~V}$. At positive potentials, the oxidation of phenols could not be ob- 

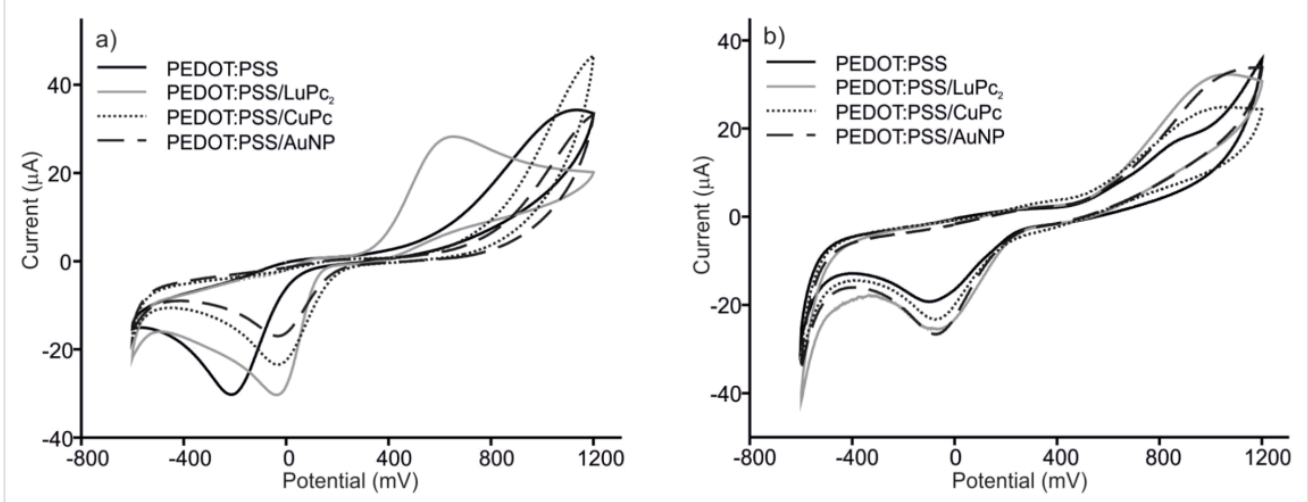

Figure 2: Cyclic voltammograms of PEDOT/PSS, PEDOT/PSS/LuPc 2 , PEDOT/PSS/CoPc and PEDOT/PSS/AuNP sensors in (a) catechol and (b) hydroquinone $1.5 \times 10^{-4} \mathrm{~mol} \cdot \mathrm{L}^{-1}$ with $0.01 \mathrm{~mol} \cdot \mathrm{L}^{-1}$ phosphate buffer as the supporting electrolyte. Scan rate $0.1 \mathrm{~V} \cdot \mathrm{s}^{-1}$.

served because it occurs at potentials higher than the working range.

In the case of PEDOT/PSS modified electrodes, catechol and hydroquinone were oxidized at the working electrode to form 1,2-benzoquinone and 1,4-benzoquinone, respectively. During the reaction, oxygen and hydrogen also form $\mathrm{H}_{2} \mathrm{O}_{2}$. During the reverse scan, quinones were reduced to the phenolic compound.

PEDOT/PSS and PEDOT/PSS/EM electrodes showed much larger current response (one order of magnitude) towards catechol than bare ITO glass. In addition, the presence of nanoparticles or phthalocyanines produced a shift of the cathodic wave to lower potentials $(-0.4 \mathrm{~V}$ in unmodified ITO, $-0.2 \mathrm{~V}$ in PEDOT/ PSS and $-0.01 \mathrm{~V}$ in PEDOT/PSS/EM). The electrocatalytic effect was stronger on the PEDOT/PSS/LuPc 2 electrode were a drastic decrease in the oxidation potential (from $1 \mathrm{~V}$ in PEDOT/ PSS to $0.65 \mathrm{~V}$ in PEDOT/PSS/LuPc 2 ) was observed. The improvement of the performance observed in PEDOT/PSS/EM, where two electrocatalytic materials are combined, can be attributed to the enhanced electron transfer rate provided by the composites due to the interactions between two electrocatalytic components. In particular, the excellent response of the $\mathrm{PEDOT} / \mathrm{PSS} / \mathrm{LuPc}_{2}$ electrode can be due to the interaction between the free radical $\mathrm{LuPc}_{2}$ and the radical intermediate produced during the oxidation of catechol. In addition, the aromatic structure of catechol can also establish strong $\pi-\pi$ interactions with phthalocyanines, thus facilitating the electron transfer.

The interaction of catechol and hydroquinone with electrodes was similar. However, a clear difference was observed with
$\mathrm{PEDOT} / \mathrm{PSS} / \mathrm{LuPc}_{2}$ electrodes where a strong decrease in the oxidation peak voltage was not observed [33]

The electrocatalytic properties of the layered composite PEDOT/PSS/EM electrodes were further investigated using electrochemical impedance spectroscopy (EIS). At $-0.5 \mathrm{~V}$, a RMS sine wave was applied with frequencies varying logarithmically from $10^{-2}$ to $10^{5} \mathrm{~Hz}$. Typical Nyquist plots obtained from a $10^{-3} \mathrm{~mol} \cdot \mathrm{L}^{-1}$ catechol solution are displayed in Figure 3 . $R_{\mathrm{S}}$ represents the solution resistance. The semicircular part of the diagram at high frequencies corresponds to electrontransfer-limited processes, and the diameter is equivalent to the electron transfer resistance $\left(R_{\mathrm{ct}}\right)$

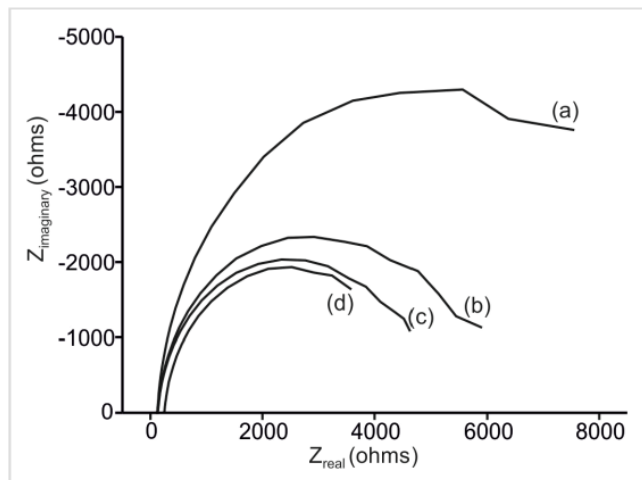

Figure 3: Nyquist plots collected at $-0.5 \mathrm{~V}$ using (a) PEDOT/PSS; (b) PEDOT/PSS/CUPc; (c) PEDOT/PSS/LuPc2; and (d) PEDOT/PSS/ AuNP. Electrodes were immersed in catechol $10^{-3} \mathrm{~mol}^{-\mathrm{L}^{-1}}$ with $0.01 \mathrm{~mol}^{-\mathrm{L}^{-1}}$ phosphate buffer $(\mathrm{pH} 7.0)$ as the supporting electrolyte. The frequency was swept logarithmically from $10^{-2}$ to $10^{5} \mathrm{~Hz}$. 
The impedance parameters were derived using a Randels equivalent circuit. The whole interface impedance was modelled by a constant phase element (CPE) (Equation 1 and Table 2):

$$
Z=\frac{1}{C_{\mathrm{CPE}}(j w) \alpha_{\mathrm{CPE}}}
$$

where $C_{\mathrm{CPE}}$ and $\alpha_{\mathrm{CPE}}$ are the capacitance and coefficient of the constant phase element, respectively.

Layered composites showed low $R_{\mathrm{ct}}$ values $(\approx 4300-5300 \Omega)$ which is about half of the $R_{\mathrm{ct}}$ observed in PEDOT/PSS $(11500 \Omega)$, indicating that charge transfer rates are higher in PEDOT/PSS/EM sensors. This agrees well with the aforementioned resistivity measurements. The smaller $R_{\mathrm{ct}}$ values revealed a low electron-transfer resistance which might be caused by the synergetic enhanced effect between PEDOT/PSS and the secondary EM.

\section{Electrochemical characterization of PEDOT/ PSS/EM enzymatic biosensors}

PEDOT/PSS/EM composites were also used as electron mediators in tyrosinase (PEDOT/PSS/EM-Tyr) and laccase-based (PEDOT/PSS/EM-Lac) biosensors. As tyrosinase is selective to the oxidation of o-diphenols and laccase catalyzes the oxidation of a larger variety of polyphenols, PEDOT/PSS/EM-Tyr sensors were used to detect catechol and PEDOT/PSS/EM-Lac sensors were used to detect hydroquinone.

In PEDOT/PSS/EM-Tyr biosensors immersed in catechol, drastic increase in intensity of the reduction peak at $-0.05 \mathrm{~V}$ was observed (Figure 4). This increase is due to the simultaneous reduction of o-quinone formed by the electrochemical oxidation at positive potentials plus the reduction of the o-quinone formed by enzymatic oxidation. The electron transfer was promoted in the presence of PEDOT/PSS and further improved in the presence of a secondary electron mediator at PEDOT/ PSS/EM electrodes. The presence of $\mathrm{LuPc}_{2}$ produced the largest increase in the peak intensity $(-30 \mu \mathrm{A}$ in PEDOT/PSS, $-80 \mu \mathrm{A}$ in PEDOT/PSS-Tyr and $-132 \mu \mathrm{A}$ in PEDOT/PSS/LuPc $c_{2}$-Tyr) No amplification was observed in the oxidation peak and the current showed the same values as those observed in nonenzymatic sensors (due to the increase in the scale, oxidation peaks cannot be clearly seen in the Figure). When biosensors containing laccase were immersed in the hydroquinone solution, a drastic increase in intensity of the reduction peak at $-0.2 \mathrm{~V}$ was also observed $(-20 \mu \mathrm{A}$ in PEDOT/PSS, $-85 \mu \mathrm{A}$ in PEDOT/ PSS-Lac and $-130 \mu \mathrm{A}$ in PEDOT/PSS/LuPc 2 -Lac). In this case, the intensity of the signal at positive potentials was also ampli-

\begin{tabular}{|c|c|c|c|c|}
\hline & $R_{\mathrm{S}}(\Omega)$ & $C_{\mathrm{CPE}}(\mathrm{F})$ & $\alpha_{C P E}$ & $R_{\mathrm{ct}}(\Omega)$ \\
\hline PEDOT/PSS & 170.8 & $1.70 \times 10^{-5}$ & 0.91 & 11430 \\
\hline PEDOT/PSS/LuPc 2 & 127.4 & $2.23 \times 10^{-5}$ & 0.92 & 4734 \\
\hline PEDOT/PSS/CuPc & 120.3 & $2.62 \times 10^{-5}$ & 0.92 & 5375 \\
\hline PEDOT/PSS/AuNPs & 148.4 & $3.2 \times 10^{-5}$ & 0.93 & 4346 \\
\hline
\end{tabular}
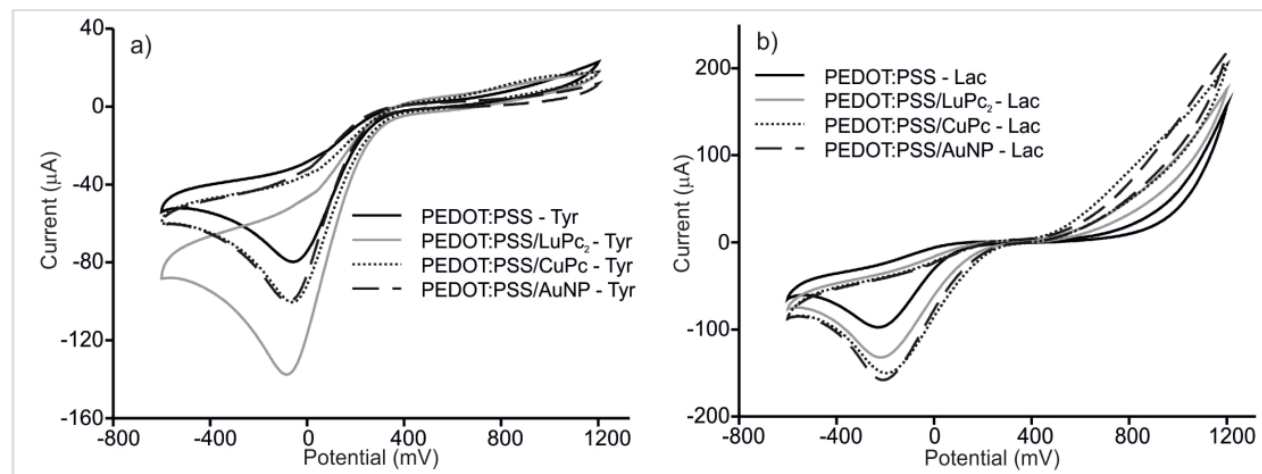

Figure 4: Cyclic voltammograms of (a) PEDOT/PSS/EM-Tyr immersed in catechol $1.5 \times 10^{-4} \mathrm{~mol} \cdot \mathrm{L}^{-1}$ and (b) PEDOT/PSS/EM-Lac immersed in hydroquinone $1.5 \times 10^{-4} \mathrm{~mol} \cdot \mathrm{L}^{-1}$. Scan rate $0.1 \mathrm{~V} \cdot \mathrm{s}^{-1}$. 
fied. The results demonstrate that the layered composites developed in this work facilitate the electron transfer and the synergistic effect between the components was evidenced.

\section{Repeatability and reproducibility}

Repetitive measurements were carried out in $10^{-3} \mathrm{~mol} \cdot \mathrm{L}^{-1}$ solutions to study the repeatability of the voltammograms. The results of five consecutive measurements showed a coefficient of variation lower than $2 \%$ in all cases. Additionally, the reproducibility of the electrodes was examined by cycling the electrodes in $10^{-3} \mathrm{~mol} \cdot \mathrm{L}^{-1}$ solutions using three electrodes prepared using the same method. The coefficient of variation in both cathodic and anodic peaks were found to be less than $4 \%$, confirming the reproducibility of the method.

It is worth mentioning that the PEDOT/PSS/EM electrodes could be used up to 50 times with a decrease in the intensity of less than $5 \%$. The PEDOT/PSS/EM enzymatic electrodes were found to be highly stable for ten scans. After that their functionality progressively decreased and could no longer be used.

\section{Scan rate dependence study}

In order to further analyze the electron transfer and to evaluate the dynamic behavior of the electrodes, voltammograms were collected at different scan rates. The responses are illustrated in Figure 5 for the PEDOT/PSS/AuNP electrode immersed in catechol $10^{-3} \mathrm{~mol} \cdot \mathrm{L}^{-1}$. The cathodic peak potentials shifted to more negative potentials with increasing scan rate (Figure 5a). This suggests the involvement of a kinetic limitation between the electrode and the phenol. The cathodic peak currents $\left(I_{\mathrm{c}}\right)$ varied linearly with the square root of the scan rate $\left(v^{1 / 2}\right)$, demonstrating the diffusion-controlled nature of the electrode reaction (Figure 5b). The slopes and correlation coefficients for the measurement of all the sensors and biosensors immersed in catechol are collected in Table 3. The results obtained in hydroquinone are presented in Table 4. According to the slope values, the reduction of hydroquinone proceeds more rapidly than that of catechol. The slope values calculated in layered PEDOT/ PSS/EM composites were higher than those observed in PEDOT/PSS, confirming the improvement of the charge transfer rates. It is also worth noting that the slopes found in
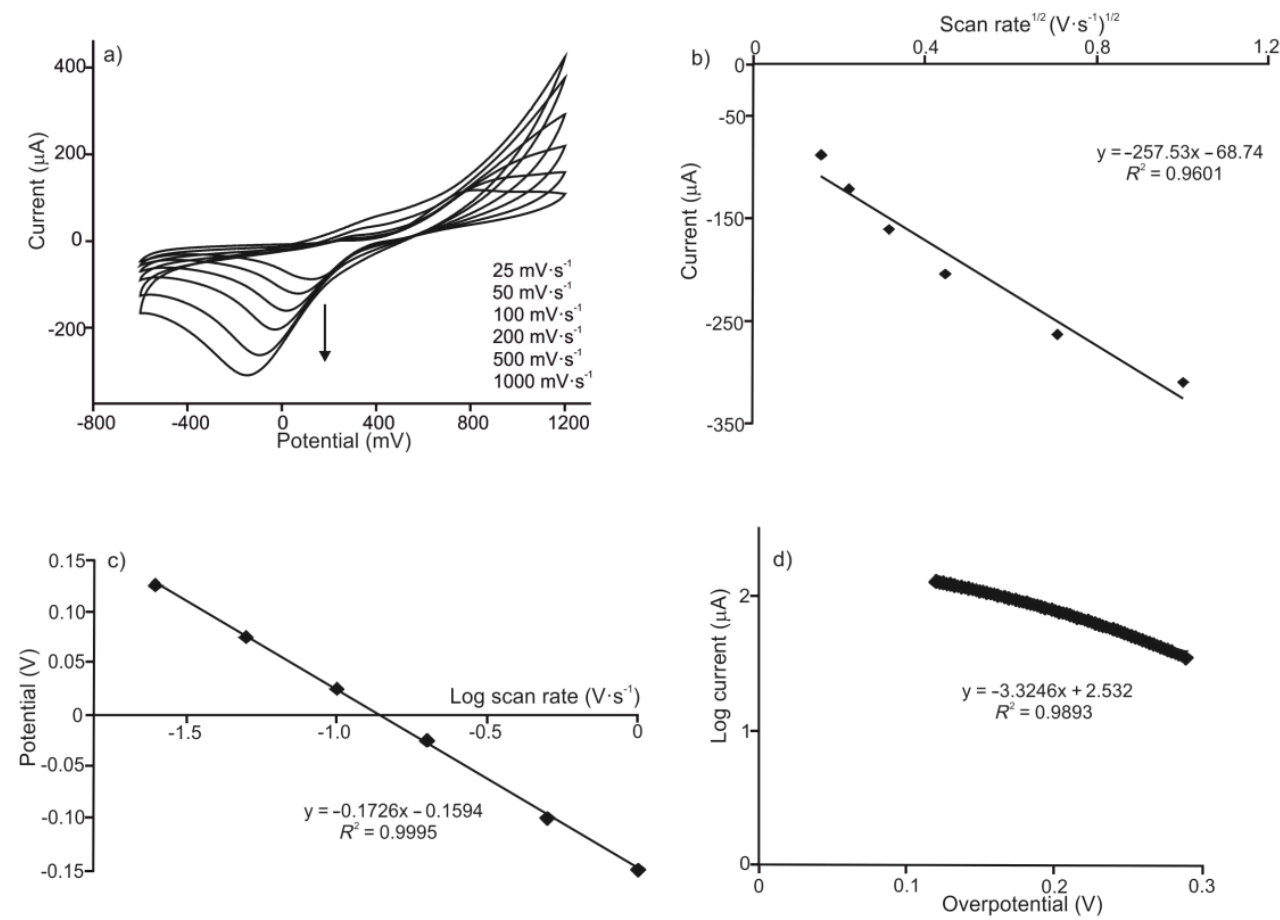

Figure 5: Effect of the scan rate in PEDOT/PSS/AuNP immersed in catechol $10^{-3} \mathrm{~mol} \cdot \mathrm{L}^{-1}$. (a) CVs collected at $0.025,0.05,0.1,0.2,0.5$ and $1.0 \mathrm{~V} \cdot \mathrm{s}^{-1} ;$ (b) linear relationship between $I_{\mathrm{c}}$ and the square root of the scan rate; (c) linear relationship between $E_{\mathrm{c}}$ and the $\log$ of the scan rate and (d) Tafel plot representing the logarithm of the intensity vs the overpotential. 
Table 3: Relationship between scan rate in sensors immersed in catechol $10^{-3} \mathrm{~mol} \cdot \mathrm{L}^{-1}$ calculated in the cathodic peak.

\begin{tabular}{llllllllll} 
& \multicolumn{2}{l}{$I_{\mathrm{c}}(\mu \mathrm{A}) \mathrm{vs} \mathrm{v}^{1 / 2}(\mathrm{~V} / \mathrm{s})^{1 / 2}$} & \multicolumn{2}{c}{$E_{\mathrm{c}}(\mathrm{V}) \mathrm{vs} \log \mathrm{v}(\mathrm{V} / \mathrm{s})$} & \multicolumn{5}{c}{$\log /(\mu \mathrm{A}) \mathrm{vs} \eta(\mathrm{V})$} \\
\hline Sensor & Slope & $R^{2}$ & Slope & $R^{2}$ & $\alpha_{n}$ & Slope & $R^{2}$ & $n$ \\
\hline PEDOT/PSS & -115.71 & 0.946 & -0.117 & 0.993 & 0.50 & -4.536 & 0.998 & 1.87 \\
PEDOT/PSS/LuPc2 & -169.33 & 0.947 & -0.144 & 0.994 & 0.41 & -4.256 & 0.991 & 1.95 \\
PEDOT/PSS/CuPc & -185.97 & 0.953 & -0.135 & 0.997 & 0.44 & -3.781 & 0.988 & 1.97 \\
PEDOT/PSS/AuNP & -257.53 & 0.960 & -0.173 & 0.999 & 0.34 & -3.324 & 0.989 & 1.73 \\
PEDOT/PSS-Tyr & -268.38 & 0.980 & -0.169 & 0.985 & 0.35 & -2.823 & 0.975 & 2.10 \\
PEDOT/PSS/LuPc2-Tyr & -309.65 & 0.977 & -0.184 & 0.992 & 0.32 & -2.361 & 0.977 & 2.30 \\
PEDOT/PSS/CuPc-Tyr & -230.25 & 0.981 & -0.174 & 0.984 & 0.34 & -2.632 & 0.968 & 2.20 \\
PEDOT/PSS/AuNP-Tyr & -231.99 & 0.984 & -0.181 & 0.984 & 0.33 & -2.402 & 0.963 & 2.30
\end{tabular}

Table 4: Relationship between scan rate in sensors immersed in hydroquinone $10^{-3} \mathrm{~mol} \cdot \mathrm{L}^{-1}$ calculated in the cathodic peak.

\begin{tabular}{llllllllll} 
& \multicolumn{2}{l}{$I_{\mathrm{c}}(\mu \mathrm{A}) \mathrm{vs}^{1 / 2}(\mathrm{~V} / \mathrm{s})^{1 / 2}$} & \multicolumn{2}{c}{$E_{\mathrm{c}}(\mathrm{V}) \mathrm{vs} \log \mathrm{v}(\mathrm{V} / \mathrm{s})$} & \multicolumn{5}{c}{$\log /(\mu \mathrm{A}) \mathrm{vs} n(\mathrm{~V})$} \\
\hline Sensor & Slope & $R^{2}$ & Slope & $R^{2}$ & $\alpha_{n}$ & Slope & $R^{2}$ & $n$ \\
\hline PEDOT/PSS & -247.88 & 0.947 & -0.168 & 0.992 & 0.50 & -4.607 & 0.997 & 1.84 \\
PEDOT/PSS/LuPc 2 & -299.33 & 0.954 & -0.199 & 0.995 & 0.41 & -3.247 & 0.999 & 2.10 \\
PEDOT/PSS/CuPc & -270.75 & 0.967 & -0.170 & 0.994 & 0.44 & -3.680 & 0.996 & 2.02 \\
PEDOT/PSS/AuNP & -300.56 & 0.966 & -0.210 & 0.993 & 0.34 & -3.391 & 0.997 & 1.70 \\
PEDOT/PSS-Lac & -411.98 & 0.975 & -0.143 & 0.988 & 0.41 & -3.050 & 0.998 & 2.3 \\
PEDOT/PSS/LuPc2-Lac & -511.99 & 0.978 & -0.206 & 0.984 & 0.29 & -2.004 & 0.999 & 2.4 \\
PEDOT/PSS/CuPc-Lac & -532.85 & 0.968 & -0.214 & 0.982 & 0.29 & -2.003 & 0.999 & 2.4 \\
PEDOT/PSS/AuNP-Lac & -463.63 & 0.969 & -0.189 & 0.990 & 0.31 & -2.254 & 0.999 & 2.3 \\
& & & & & & & & &
\end{tabular}

enzymatic PEDOT/PSS/EM biosensors were higher than the values found in nonenzymatic PEDOT/PSS/EM electrodes This result indicates that the charge transfer within the composite film and/or through the electrode interface is facilitated in biosensors and this improvement should be related with the enzymatic activity.

In the scan rates ranging from 0.025 to $1 \mathrm{~V} \cdot \mathrm{s}^{-1}$, the cathodic peak potential $\left(E_{\mathrm{c}}\right)$ showed a linear relationship with the logarithm of scan rate $(\log v)$ (Figure $5 \mathrm{c}$ ) according to Laviron's equation (Equation 2) for a totally irreversible diffusion-controlled process $[34,35]$.

$$
E_{\mathrm{c}}=E^{0}-\frac{2.3 R T}{\left(\alpha_{\mathrm{c}}\right) n F} \log \mathrm{v}
$$

where $\alpha$ is the transfer coefficient, $v$ is the scan rate (expressed The simplified Butler-Volmer equation can be applied to calcuin $\mathrm{V} \cdot \mathrm{s}^{-1}$ ), $n$ is the number of electrons involved in the rate-de- late the $\alpha$ values (Equation 3) [35]. The Tafel slope was calcu-

termining step, $R$ is the ideal gas constant $\left(8314 \mathrm{~J} \cdot \mathrm{mol}^{-1} \cdot \mathrm{K}^{-1}\right), \quad$ lated from the representation of $\log I \mathrm{vs}$ the overpotential ( $\eta$ )
$T$ is the temperature $(298 \mathrm{~K})$ and $F$ is Faraday's constant $\left(95,484.56 \mathrm{C} \cdot \mathrm{mol}^{-1}\right)$

The regression coefficients calculated for the analysis of all the sensors and biosensors immersed in catechol and hydroquinone are presented in Table 3 and Table 4, respectively.

The linear variation of the peak potential, $E_{\mathrm{c}}$, as a function of $\log v$ suggests that the electrode process can be regarded as a totally irreversible reaction. From the slope of the graph, $\alpha_{n}$ can be determined, where $\alpha$ is the transfer coefficient and $n$ is the number of electrons transferred in the rate-determining step.

The slope indicated that the values $\alpha_{n}$ have values between $0.3-0.5$ for the cathodic peak. These values indicate the total irreversibility of the electron transfer process and also confirm the ideal diffusion-controlled mechanism [36]. 
obtained from a voltammogram collected in catechol or hydroquinone $10^{-3} \mathrm{~mol} \cdot \mathrm{L}^{-1}$ at a scan rate of $0.1 \mathrm{~V} \cdot \mathrm{s}^{-1}$ (Figure $5 \mathrm{~d}$ ) Then, using the results obtained from the Laviron equation for $\alpha_{n}$, the number of electrons transferred can be calculated.

$$
\log I=\log I_{0}-\frac{\alpha F}{2.3 R T} \eta
$$

The calculated Tafel slopes, the $\alpha$ values and the number of electrons are listed in Table 3 and Table 4 . As can be seen from these results, the Tafel slopes showed a less negative value in the electrodes modified with the composite PEDOT/PSS/EM The values were even lower in the presence of an enzyme, confirming the improved electrocatalytic activity. This resul further confirms that the combination of PEDOT/PSS with AuNPs and phthalocyanines can promote electron transfer in the active centers of biological molecules, increasing the relative activity of the enzymes

The number of electrons involved in the reaction calculated from the Tafel slope and Equation 2 confirms a two electron process in all electrodes, confirming the proposed mechanism.

\section{Limit of detection}

The limit of detection (LD) was evaluated by analyzing the response of the sensors towards phenol solutions with concentrations ranging from $4.0 \times 10^{-6} \mathrm{~mol} \cdot \mathrm{L}^{-1}$ to $1.5 \times 10^{-4} \mathrm{~mol} \cdot \mathrm{L}^{-1}$ The results are illustrated in Figure 6 for PEDOT/PSS/LuPc and PEDOT/PSS/LuPc 2 -Tyr immersed in catechol.
In PEDOT/PSS/EM electrodes, a linear relationship was observed between the current response signals and the concentration of catechol and hydroquinone, confirming that they may reliably be used for the determination of both compounds in this concentration range. The limit of detection calculated from the anodic peak $\left(\mathrm{LD}_{\mathrm{A}}\right)$ and the cathodic peak $\left(\mathrm{LD}_{\mathrm{C}}\right)$ (using the $3 \sigma$ criteria) and correlation coefficients are collected in Table 5.

The results confirmed that the LD towards catechol was improved almost one order of magnitude when using layered composite electrodes and attained the $\mu \mathrm{mol} \cdot \mathrm{L}^{-1}$ range. The improvement was not so marked in hydroquinone, but also in this case, the LD calculated from the cathodic peak was in the $\mu \mathrm{mol} \cdot \mathrm{L}^{-1}$ range.

The improved performance can be attributed to synergistic interactions between PEDOT/PSS and the additives similar to the interactions already described between PEDOT/PSS and graphene [37]. In our composites, PEDOT/PSS acts as the electron mediator and the additive further enhances the electron transfer due to the high electrical conductivity demonstrated in the impedance experiments.

In the case of PEDOT/PSS/EM enzymatic biosensors, the main amplification occurred in the cathodic wave. For this reason, the study of the LD was carried out only in the cathodic reduction peak.

When representing the peak current vs catechol concentration obtained from PEDOT/PSS/EM-Tyr, a linear relationship was obtained in the concentration range from $4.0 \times 10^{-6}$ to
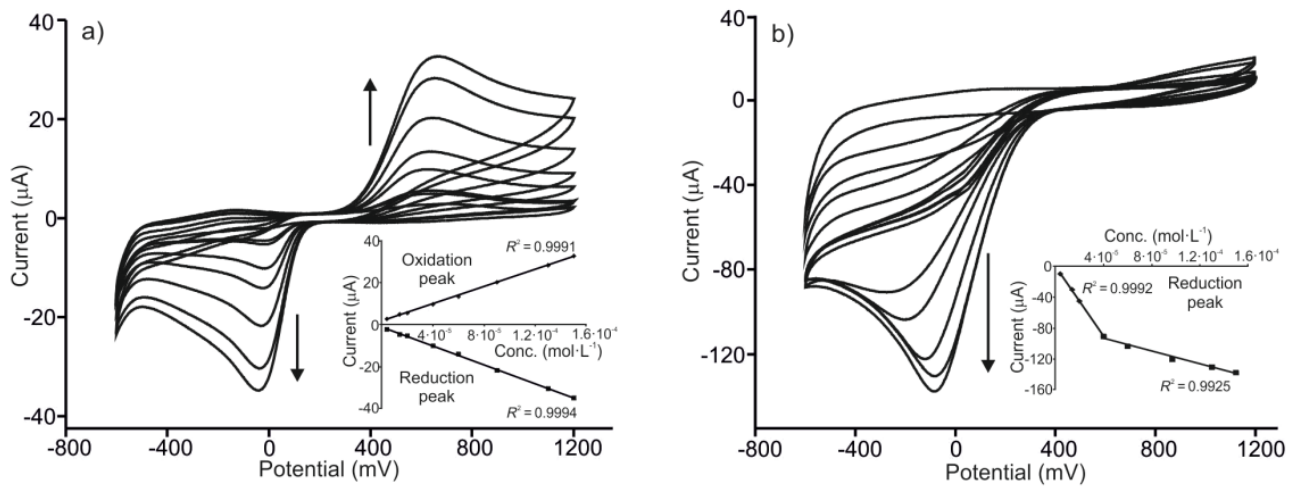

Figure 6: CVs of (a) PEDOT/PSS/LuPc 2 and (b) PEDOT/PSS/LuPc $c_{2}$-Tyr immersed in increasing concentrations of catechol (from $4.0 \times 10^{-6} \mathrm{~mol}^{\mathrm{L}} \mathrm{L}^{-1}$ to $\left.1.5 \times 10^{-4} \mathrm{~mol} \cdot \mathrm{L}^{-1}\right)$. Scan rate $0.1 \mathrm{~V} \cdot \mathrm{s}^{-1}$ 
Table 5: Limit of detection and regression coefficients obtained for catechol and hydroquinone using the anodic (LD $\left(L D_{A}\right)$ and the cathodic (LD $)$ peaks.

\begin{tabular}{lllll} 
Sensor & $\mathrm{LD}_{\mathrm{A}}\left(\mathrm{mol} \cdot \mathrm{L}^{-1}\right)$ & $R^{2}$ (anode) & $\mathrm{LD}_{\mathrm{C}}$ (mol. $\mathrm{L}^{-1}$ ) & $R^{2}$ (cathode) \\
\hline Catechol & & & & \\
\hline PEDOT/PSS & $4.96 \times 10^{-5}$ & 0.993 & $6.58 \times 10^{-6}$ & 0.998 \\
PEDOT/PSS/LuPc 2 & $1.23 \times 10^{-6}$ & 0.999 & $1.03 \times 10^{-6}$ & 0.999 \\
PEDOT/PSS/CuPc & $2.99 \times 10^{-6}$ & 0.992 & $1.81 \times 10^{-6}$ & 0.996 \\
PEDOT/PSS/AuNP & $2.18 \times 10^{-6}$ & 0.998 & $0.87 \times 10^{-6}$ & 0.999 \\
\hline Hydroquinone & & & & \\
\hline PEDOT/PSS & & & & 0.970 \\
PEDOT/PSSLuPc2 & $2.85 \times 10^{-5}$ & 0.971 & $1.61 \times 10^{-5}$ & 0.997 \\
PEDOT/PSS/CuPc & $1.80 \times 10^{-5}$ & 0.996 & $1.51 \times 10^{-6}$ & 0.998 \\
PEDOT/PSS/AuNP & $2.69 \times 10^{-5}$ & 0.972 & $7.55 \times 10^{-6}$ & 0.996 \\
& $1.97 \times 10^{-5}$ & 0.993 & &
\end{tabular}

$6 \times 10^{-5} \mathrm{~mol} \cdot \mathrm{L}^{-1}$. Then, a change in the slope occurred and a new linear range was observed from $9.0 \times 10^{-5}$ to $1.5 \times 10^{-4} \mathrm{~mol} \cdot \mathrm{L}^{-1}$. The limit of detection calculated in both linear ranges is shown in Table 6. A LD in the low concentration range was attained $\left(10^{-7} \mathrm{~mol} \cdot \mathrm{L}^{-1}\right)$, that is, one order of magnitude lower than the LD obtained in the nonenzymatic sensor. The LD in the high concentration scale were similar to those obtained for the nonenzymatic sensors and were in the $\mu \mathrm{mol} \cdot \mathrm{L}^{-1}$ range. The obtained LDs were similar to values reported in recent works using tyrosinase and graphene as electron mediator $[38,39]$. The LD obtained for hydroquinone was also one order of magnitude lower than in nonenzymatic sensors and attained values of $10^{-6} \mathrm{~mol} \cdot \mathrm{L}^{-1}$.

\section{Conclusion}

Owing to their unique electrochemical properties, layered composite PEDOT/PSS/EM and PEDOT/PSS/EM-Enz electrodes offer efficient electron transfer, low detection limit, high sensitivity and good reproducibility toward the oxidation of catechol and hydroquinone.

Furthermore, the combination of PEDOT with AuNPs or phthalocyanines can promote electron transfer in the active centers of biological molecules, increasing the relative activity of the enzymes. The linear relationship between the peak current and the square root of the scan rate for both PEDOT/PSS/ EM sensors and PEDOT/PSS/EM-Enz biosensors in catechol

Table 6: Limit of detection and regression coefficients obtained for catechol and hydroquinone using the cathodic (LDO) peaks using biosensors.

\begin{tabular}{|c|c|c|c|c|}
\hline Sensor & $\mathrm{LD}_{\mathrm{C}}\left(\mathrm{mol} \cdot \mathrm{L}^{-1}\right)$ & & $R^{2}$ (cathode) & \\
\hline Conc. range & $4 \times 10^{6}-6 \times 10^{-5}$ & $9 \times 10^{-5}-1.5 \times 10^{-4}$ & $4 \times 10^{-6}-6 \times 10^{-5}$ & $9 \times 10^{-5}-1.5 \times 10^{-4}$ \\
\hline \multicolumn{5}{|l|}{ Catechol } \\
\hline PEDOT/PSS-Tyr & $6.62 \times 10^{-7}$ & $6.55 \times 10^{-6}$ & 0.9862 & 0.987 \\
\hline PEDOT/PSS/LuP(2-Tyr & $4.62 \times 10^{-7}$ & $2.53 \times 10^{-6}$ & 0.9925 & 0.999 \\
\hline PEDOT/PSS/CuPc-Tyr & $4.37 \times 10^{-7}$ & $3.16 \times 10^{-6}$ & 0.9971 & 0.936 \\
\hline PEDOT/PSS/AuNP-Tyr & $3.88 \times 10^{-7}$ & $2.80 \times 10^{-6}$ & 0.9889 & 0.971 \\
\hline \multicolumn{5}{|l|}{ Hydroquinone } \\
\hline PEDOT/PSS-Lac & $6.00 \times 10^{-6}$ & & 0.997 & \\
\hline PEDOT/PSS/LuPc $c_{2}$ Lac & $1.11 \times 10^{-6}$ & & 0.996 & \\
\hline PEDOT/PSS/CUPc-Lac & $1.52 \times 10^{-6}$ & & 0.999 & \\
\hline PEDOT/PSS/AuNP-Lac & $2.51 \times 10^{-6}$ & & 0.998 & \\
\hline
\end{tabular}


and hydroquinone indicate a diffusion-controlled analyte electrocatalytic oxidation, and Tafel slopes confirmed the improved electrocatalytic activity. The modified electrodes designed here show excellent limits of detection that are comparable with those found in literature. The reported sensors could be used to analyze the phenolic content of foods and beverages such as wine, must, coffee, saffron, etc.

\section{Experimental \\ Reagents and solutions}

Poly(3,4-ethylenedioxythiophene)/poly(styrene sulfonate) (PEDOT/PSS) aqueous solution $\left(3.0-4.0 \%\right.$ in $\mathrm{H}_{2} \mathrm{O}$, high conductivity grade) and copper(II) phthalocyanine tetrasulfonic acid tetrasodium salt $(\mathrm{CuPc}, 0.05 \mathrm{~g} / \mathrm{L})$ were purchased from Sigma-Aldrich. Gold nanoparticle (AuNPs, 30-40 nm) colloids were synthesized according to a modification of the procedure proposed by Slot and Geuze $[12,40]$. Using this procedure, a red colloid with a UV absorbance maximum at $\lambda=540 \mathrm{~nm}$ was obtained. The lutetium(III) bisphthalocyaninate $\left(\mathrm{LuPc}_{2}, 0.05 \mathrm{~g} / \mathrm{L}\right)$ was synthesized following a previously published procedure [41].

Phenol oxidase enzymes were purchased from Sigma-Aldrich tyrosinase (from mushroom, activity $\geq 1000 \mathrm{U} \cdot \mathrm{mg}^{-1}$ ) and laccase (from Trametes versicolor, activity $\geq 10 \mathrm{U} \cdot \mathrm{mg}^{-1}$ ) $5 \mathrm{mg} \cdot \mathrm{L}^{-1}$ solutions of tyrosinase and laccase were prepared in buffered phosphate $0.01 \mathrm{~mol} \cdot \mathrm{L}^{-1}(\mathrm{pH} 7.0)$

\section{Preparation of the electrochemical sensors and biosensors}

Sensors were prepared using a spin coater, model $1 \mathrm{H}-\mathrm{D} 7$ (Micasa Co., Tokyo, Japan). ITO glass substrates $\left(1 \mathrm{~cm}^{2}\right.$ surface area) were used as the substrate. Prior to the film deposition, the substrates were washed in an ultrasonic bath with acetone and rinsed twice with deionized water (MilliQ)

PEDOT/PSS was diluted 1:10 in deionized water and stirred in an ultrasonic bath for $10 \mathrm{~min}$. Then, $100 \mu \mathrm{L}$ of the solution was dropped onto the ITO glass and spin-coated at $2000 \mathrm{rpm}$ for $120 \mathrm{~s}$ (slope of $120 \mathrm{~s}$ ). The PEDOT/PSS films obtained were annealed at $150^{\circ} \mathrm{C}$ for $15 \mathrm{~min}$

PEDOT/PSS/EM layered sensors were prepared on the surface of PEDOT/PSS films by spin coating $200 \mu \mathrm{L}$ of $\mathrm{CuPc}$ and AuNPs, or $100 \mu \mathrm{L}$ of $\mathrm{LuPc}_{2}$ at $2000 \mathrm{rpm}$ for $120 \mathrm{~s}$ (slope of $120 \mathrm{~s}$ ), followed by annealing at $150^{\circ} \mathrm{C}$ for $15 \mathrm{~min}$.

PEDOT/PSS/EM enzymatic biosensors were prepared by depositing laccase or tyrosinase onto the PEDOT/PSS/EM sensors. For this purpose, $50 \mu \mathrm{L}$ of $0.01 \mathrm{~mol} \cdot \mathrm{L}^{-1}$ phosphate buffer ( $\mathrm{pH} 7.0$ ) containing $5 \mathrm{mg} \cdot \mathrm{mL}^{-1}$ of enzyme were deposited onto the PEDOT/PSS/EM electrode. After drying at room temperature for approximately $45 \mathrm{~min}$., the biosensors were immersed in glutaraldehyde $(2.5 \% \mathrm{v} / \mathrm{v}$, buffer solution) for $5 \mathrm{~min}$ and dried in air for $15 \mathrm{~min}$ at room temperature. The biosensors were then rinsed with phosphate buffer to remove any unbound enzyme and stored at $4{ }^{\circ} \mathrm{C}$.

\section{Characterization of the sensors}

Scanning electron microscopy (SEM) (FEI, QUANTA 200F) was used to record the images of the electrode surfaces. A square resistance was measured using a four-point tester (HAAMEG, HM 8040-2).

Electrochemical impedance spectroscopy (EIS) experiments were performed using a Solartron impedance analyzer. The measurements were carried out by applying a signal amplitude of $10 \mathrm{mV}$, at a working potential of $-0.5 \mathrm{~V}$ with frequencies varied logarithmically from $0.1 \mathrm{~Hz}$ to $100 \mathrm{kHz}$. The measurements were made by immersing the electrode in $10^{-3} \mathrm{~mol} \cdot \mathrm{L}^{-1}$ catechol in 0.01 buffer phosphate ( $\mathrm{pH} 7$ ). The impedance spectra were fitted with the aid of the Zview 2 software.

Voltammetric measurements were obtained using a potentiostat galvanostat PGSTAT128 (Autolab Metrohm, Utrecht, Netherlands). The electrochemical cell was a three-electrode system using the corresponding PEDOT/PSS, PEDOT/PSS/EM or PEDOT/PSS/EM-Enz modified electrode as the working electrode; the reference electrode was $\mathrm{Ag} \mid \mathrm{AgCl} / \mathrm{KCl} 3 \mathrm{~mol} / \mathrm{L}$ and the counter electrode was a platinum sheet with a surface area of $1 \mathrm{~cm}^{2}$.

Cyclic voltammetry was carried out from $-0.6 \mathrm{~V}$ to $+1.2 \mathrm{~V}$ (vs $\mathrm{Ag} / \mathrm{AgCl}$ ) with a scan rate of $0.1 \mathrm{~V} \cdot \mathrm{s}^{-1}$, except when indicated otherwise. A phosphate buffer solution $\left(0.01 \mathrm{~mol} \cdot \mathrm{L}^{-1}\right.$; $\left.\mathrm{pH} 7.0\right)$ was employed as the electrolytic medium in electroanalysis experiments. The influence of the potential sweep rate was studied in $10^{-3} \mathrm{~mol} \cdot \mathrm{L}^{-1}$ catechol or $10^{-3} \mathrm{~mol} \cdot \mathrm{L}^{-1}$ hydroquinone in 0.01 phosphate buffer ( $\mathrm{pH} 7$ ), while varying the scan rates from 0.025 to $1.0 \mathrm{~V} \cdot \mathrm{s}^{-1}$

The limits of detection (LD) was calculated from peak current responses taken from voltammograms recorded at different concentrations from $4 \cdot 10^{-6}-1.5 \cdot 10^{-4} \mathrm{~mol} / \mathrm{L}$, following the " $3 \mathrm{sd} / \mathrm{m}$ " criterion, where " $\mathrm{m}$ " is the slope of the calibration graph and "sd" was estimated as the standard deviation $(n=5)$ of the voltammetric signal at the concentration level corresponding to the lowest concentration of the calibration plot.

\section{Acknowledgements}

Financial support by MINECO-FEDER (AGL2015-67482-R) and the Junta de Castilla y Leon FEDER (VA-011U16) is grate- 
fully acknowledged. Celia Garcia-Hernandez would also like to thank Junta de Castilla y León for a grant (BOCYL-D-41120159).

\section{References}

1. Makhotina, O.; Kilmartin, P. A. Anal. Chim. Acta 2010, 668, 155-165. doi:10.1016j.aca.2010.03.064

2. Yang, C.; Denno, M. E.; Pyakurel, P.; Venton, B. J. Anal. Chim. Acta 2015, 887, 17-37. doi:10.1016/.aca.2015.05.049

3. Lange, U.; Roznyatovskaya, N. V.; Mirsky, V. M. Anal. Chim. Acta 2008, 614, 1-26. doi:10.1016j.aca.2008.02.068

4. He, K.; Wang, X.; Meng, X.; Zheng, H.; Suye, S.-i. Sens. Actuators, $B$ 2014, 193, 212-219. doi:10.1016/.snb.2013.11.055

5. Huo, Z.; Zhou, Y.; Liu, Q.; He, X.; Liang, Y.; Xu, M. Microchim. Acta 2011, 173, 119-125. doi:10.1007/s00604-010-0530-y

6. Medina-Plaza, C.; Rodriguez-Mendez, M. L.; Sutter, P.; Tong, X: Sutter, E. J. Phys. Chem. C 2015, 119, 25100-25107. doi: $10.1021 /$ acs.jpcc.5b07960

7. Lin, X.; Ni, Y.; Kokot, S. Anal. Chim. Acta 2013, 765, 54-62. doi:10.1016/.aca.2012.12.036

8. Alessio, P.; Pavinatto, F. J.; Oliveira, O. N., Jr.; de Saja Saez, J. A. Constantino, C. J. L.; Rodriguez-Méndez, M. L. Analyst 2010, 135, 2591-2599. doi:10.1039/c0an00159g

9. Zagal, J. H.; Griveau, S.; Silva, J. F.; Nyokong, T.; Bedioui, F. Coord. Chem. Rev. 2010, 254, 2755-2791. doi:10.1016/.ccr.2010.05.001

10. Apetrei, C.; Casilli, S.; De Luca, M.; Valli, L.; Jiang, J. Rodríguez-Méndez, M. L.; De Saja, J. A. Colloids Surf, A 2006, 284-285, 574-582. doi:10.1016/.colsurfa.2005.10.069

11. Yoshioka, Y.; Jabbour, G. E. Synth. Met. 2006, 156, 779-783. doi:10.1016/.synthmet.2006.03.013

12. García-Hernández, C.; García-Cabezón, C.; Medina-Plaza, C.; Martín-Pedrosa, F.; Blanco, Y.; de Saja, J.A.;

Rodríguez-Méndez, M. L. Beilstein J. Nanotechnol. 2015, 6 , 2052-2061. doi:10.3762/ bjnano.6.209

13. Medina-Plaza, C.; Furini, L. N.; Constantino, J. C. L.; de Saja, J. A.; Rodriguez-Mendez, M. L. Anal. Chim. Acta 2014, 851, 95-102. doi:10.1016j.aca.2014.08.049

14. Mathiyarasu, J.; Senthilkumar, S.; Phani, K. L. N.; Yegnaraman, V. Mater Left 2008, 62, 571-573, doi:101016/j matlet 200706.004

15. Dutse, S. W.; Yusof, N. A.; Ahmad, H. Sens. Transducers J. 2015, 191 $46-51$

16. Pingarrón, J. M.; Yáñez-Sedeño, P.; González-Cortés, A. Electrochim. Acta 2008, 53, 5848-5866. doi:10.1016/j.electacta.2008.03.005

17. Reis, R. M.; Valim, R. B.; Rocha, R. S.; Lima, A. S.; Castro, P. S.; Bertotti, M.; Lanza, M. R. V. Electrochim. Acta 2014, 139, 1-6. doi:10.1016/j.electacta.2014.07.003

18. Rodriguez-Méndez, M. L.; Gay, M.; de Saja, J. A J. Porphyrins Phthalocyanines 2009, 13, 1159-1167. doi:10.1142/S1088424609001509

19. Cortina-Puig, M.; Noguer, T.; Marty, J.-L.; Calas-Blanchard, C. Electrochemical Biosensors as a Tool for the Determination of Phenolic Compounds and Antioxidant Capacity in Foods and Beverages. In Biosensors in Food Processing, Safety and Quality Control; Mutlu, M., Ed.; CRC Press: Boca Raton, FL, 2011. doi:10.1201/b10466-11

20. Karim, F.; Fakhruddin, A. N. M. Rev. Environ. Sci. Bio/Technol. 2012, 11, 261-274. doi:10.1007/s11157-012-9268-9
21. Lupu, S: Lete C. Balaure, P. C.: Caval, D. I. Mihailciuc, C. Lakard, B.; Hihn, J.-Y.; del Campo, F. J. Sensors 2013, 13, 6759-6774. doi:10.3390/s130506759

22.Manesha, K. M.; Santhosha, P.; Gopalana, A.; Le, K. P. Talanta 2008 75, 1307-1314. doi:10.1016/j.talanta.2008.01.038

23.Yin, H. S.; Zhou, Y. L.; Xu, J.; Ai, S. Y.; Cui, L.; Zhu, L. S. Anal. Chim. Acta 2010,659, 144-150. doi:10.1016/.aca.2009.11.051

24.Fernandes, E. G. R.; Brazaca, L. C.; Rodríguez-Mendez, M. L de Saja, J. A.; Zucolotto, V. Biosens. Bioelectron. 2011, 26 , 4715-4719. doi:10.1016/.bios.2011.05.032

25.Apetre, C.; Alessio, P.; Constantino, C. J. L.; de Saja, J. A. Rodriguez-Mendez, M. L.; Pavinatto, F. J.; Ramos Fernandes, E. G Zucolotto, V.; Oliveira, O. N., Jr. Biosens. Bioelectron. 2011, 26 2513-2519. doi:10.1016/.bios.2010.10.047

26.Apetrei, I. M.; Rodriguez-Mendez, M. L.; Apetrei, C.; de Saja, J. A. Sens. Actuators, B 2013, 177, 138-144. doi:10.1016/j.snb.2012.10.131

27. Roleira, F. M. F.; Tavares-da-Silva, E. J.; Varela, C. L.; Costa S. C

Silva, T.; Garrido, J.; Borge, F. Food Chem. 2015, 183, 235-258. doi: 10.1016/j.foodchem.2015.03.039

28. Pradeep, N. V.; Anupama, S.; Navya, K.; Shalini, H. N.; Idris, M.; Hampannavar, U. S. Appl. Water Sci. 2015, 5, 105-112. doi: 10.1007/s13201-014-0176-8

29. Ouyang, J.; Chu, C.-W.; Chen, F.-C.; Xu, Q.; Yang, Y. Adv. Funct. Mater. 2005, 15, 203-208. doi:10.1002/adfm.200400016

30.Nevrela, J.; Micjan, M.; Novota, M.; Kovacova, S.; Pavuk, M.; Juhasz, P.; Kocac, J., Jr.; Jakaboric, J.; Weis, M. J. Polym. Sci., Part B: Polym. Phys. 2015, 53, 1139-1146. doi: 10.1002/polb.23754

31.Crispin, X.; Marciniak, C.; Osikowicz, W.; Zotti, G.; Denier van der Gon, A. W.; Louwet, F.; Fahlman, M.; Groenedaal, L.; De Schryver, F.; Salanek, W. R. J. Polym. Sci, Part B: Polym. Phys. 2003, 41, 2561-2583. doi:10.1002/polb.10659

32. Bouvet, M.; Gaudillat, P.; Suisse, J.-M. J. Porphyrins Phthalocyanines 2013, 17, 913-919. doi:10.1142/S1088424613300073

33.Ma, L.; Wang, Z.; Li, Q. Analyst 2012, 137, 432-436. doi: 10.1039/C1AN15865A

34. Bard, A. J.; Faulkner, L. R. Electrochemical Methods: Fundamentals and Applications, 2nd ed.; John Wiley and Sons: New York, 2001.

35.Zhang, J.; Xu, J.; Wen, Y.; Wang, Z.; Zhang, H.; Ding, W. Electroanal. Chem. 2015, 751, 65-74 doi: 10.1016/.jelechem.2015.05.032

36. Alipour, E.: Majidi, M. R.: Saadatirad, A.; Golabi, S. M. Anal. Methods 2012, 4, 2288-2295. doi:10.1039/c2ay25463h

37.Sriprachuabwong, C.; Karuwan, C.; Wisitsorrat, A.; Phokharatkul, D. Lomas, T.; Sritongkham, P.; Tuantranont, A. J. Mater. Chem. 2012, 22 5478-5485. doi:10.1039//2jm14005e

38. Sethuraman, V.; Muthuraja, P.; Ananda Raj, J.; Manisankar, P. Biosens. Bioelectron. 2016, 84, 112-119. doi: 10.1016/.bios.2015.12.074

39. Wu, L.; Deng, D.; Jin, J.; Lu, X.; Chen, J. Biosens. Bioelectron. 2012 35, 193-199. doi:10.1016/j.bios.2012.02.045

40.Slot, J. W.; Geuze, H. J. J. Cell Biol. 1981, 90, 533-536. doi: $10.1083 / \mathrm{jcb} .90 .2 .533$

41. Linaje, M.; Quintanilla, M. C.; González, A.; del Valle, J. L.; Alcaide, G.; Rodríguez-Méndez, M. L. Analyst 2000, 125, 341-346. doi: $10.1039 / a 908398 \mathrm{~g}$ 


\section{Chapter 3: Results and discussion}

Paper VI: Layered composites of PEDOT:PSS/nanoparticles and PEDOT:PSS/phthalocyanines as electron mediators for sensors and biosensors.

Beilstein J. Nenotechnol. 2016, 7, 1948-1959.

\section{License and Terms}

This is an Open Access article under the terms of the Creative Commons Attribution License

(http://creativecommons.org/licenses/by/4.0), which permits unrestricted use, distribution, and reproduction in any medium, provided the original work is properly cited.

The license is subject to the Beilstein Journal of

Nanotechnology terms and conditions:

(http://www.beilstein-journals.org/bjnano)

The definitive version of this article is the electronic one which can be found at:

doi: $10.3762 /$ bjnano. 7.186 


\subsection{Paper VII}

Impedimetric electronic tongue based on nanocomposites for the analysis of red wines. Improving the variable selection method.

By Celia García-Hernández, Coral Salvo-Comino, Fernando Martín-

Pedrosa, María Luz Rodríguez-Méndez and Cristina García-Cabezón.

Sensors and Actuators B 277 (2018) 365-372.

(doi: 10.1016/j.snb.2018.09.023) 


\subsubsection{Motivation and objective}

Electrochemical sensors implemented in the technology of electronic tongues are usually potentiometric, voltammetric or ampererometric. However, research works focused on impedimetric sensors applied in ET are scarce compared to the other electrochemical sensors. Moreover, impedimetric electronic tongues implemented for food authenticity have not been widely applied and only Riul et al. have reported research work in which they used an impedimetric ET to analyze different brands of red wines. Furthermore, Riul and co-workers have actively implemented these devices, being the first to develop an array of sensors based on LB films containing conducting polymers to assess brands of mineral water, tea and coffee.

Impedimetric sensors measure the impedance using impedance spectroscopy at a constant frequency or a full frequency spectrum. The use of these sensors can provide some advantages with respect to the most commonly used electrochemical sensors. For instance, in terms of taste sensors for liquid media, potentiometric sensors have limited sensitivity in nonelectrolyte compounds, whereas voltammetric devices need redox species in the media and the responses are quite complex and require advanced statistical treatments. Moreover, impedimetric devices can be used in any kind of solution, even without electrical conductivity, and do not need a reference electrode to carry out the measurements, thus facilitating the data acquisition because a reliable reference electrode is a very important factor in many practical applications.

Basically, impedimetric electronic tongues have used to test different mineral waters, to study water contaminated with heavy metals, to detect trace levels of phenolic compounds in water, to analyze organophosphate pesticides and discriminate between different wines, to detect tetracycline residue in milk samples and discriminate the five classic tastes.

In a previous research work, we developed an array of sensors based on the conducting polymer PEDOT:PSS modified with different sensing materials that improved the electron transfer. PEDOT:PSS/LUPC2 and PEDOT:PSS/AuNP composite sensors were identified as the most reproducible and sensitive sensors. For this reason, in this work, we have selected these sensors along with the non-modified sensor of PEDOT:PSS to form an impedimetric electronic tongue. The multisensor system has been applied to discriminate red wines with similar characteristics (same region, vintage and ageing method) but different variety. The novelty of this work consists in developing an improved impedimetric e-tongue applied to the analysis of red 
wines. For this purpose, we have carried out different strategies to find the most suitable method that will offer a better discrimination capability, including the classical method of selecting resistance and capacitance data at a fixed frequency, selecting parameters of the Nyquist and Bode plots obtained in electrochemical impedance spectroscopy (EIS) analysis, and finally, by fitting the results obtained in EIS expriments to an equivalent electric circuit.

The objective of this work was to develop an improved impedimetric e-tongue for the analysis of red wines, for which two strategies were followed. On the one hand, to develop new impedimetric sensors based on combinations of well-known electrocatalytic materials, including PEDOT:PSS, gold nanoparticles and phthalocyanines. On the other hand, the impedance spectroscopy measurements used for impedimetric e-tongues are diverse, in some cases measurements of electrical resistance and/or capacitance have been used at a fixed frequency value. Here, different possibilities were tested, including the fitting by means of an equivalent circuit to determine which method offers the best discrimination capability.

\subsubsection{Summary of the research work}

The sensors PEDOT:PSS, PEDOT:PSS/LUPC 2 and PEDOT:PSS/AuNPS were prepared on an ITO surface, as already described, by using the spin-coating technique.

The red wines analyzed were elaborated by the Oenological Centre of Rueda (Valladolid, Castilla y León) and they also provided the chemical parameters of total polyphenolic index (TPI), acidity, $\mathrm{SO}_{2}$ content, reducing sugars, glucose and fructose content, alcoholic degree and $\mathrm{pH}$. In addition, the Folin-Ciocalteu method was carried out to evaluate the phenolic content by means of another methodology different from TPI. Wines were harvested in different cellars of the same Denomination of Origin (D.O.), vinified with the same method and aged for 6 months in oak barrel and 18 months in bottle. The red wine samples were: three samples from the variety of grape Tempranillo, one from the variety Tinta de Toro (a clone of Tempranillo), and the third from a mixture of Tempranillo (90\%) and Garnacha (10\%).

Firstly, and before analyzing the red wines, the sensors were characterized by testing the conductivity and capacitance and carrying out electrochemical impedance spectroscopy measurements (EIS). 
Conductivity results evidenced that it increased for the sensors based on modified PEDOT:PSS sensors and, therefore, the values were higher for PEDOT:PSS/LUPC2 followed by PEDOT:PSS/AuNPS. These results were related to the intimate contact between the conducting polymer chains with the charge carriers of the intrinsic semiconductor $\mathrm{LuPC}_{2}$ and due to the enlargement of the effective surface area caused by the AuNPs, along with the existence of mixed valence states on their surface. Capacitance vs. voltage plots showed a characteristic $p$ doped behavior with a slight decrease at low potentials and then a steep rise at intermediate potentials. Further, carrier concentrations $\left(\mathrm{N}_{\mathrm{D}}\right)$ and flat band potentials $\left(\mathrm{V}_{\mathrm{FB}}\right)$ of the samples were obtained by Mott-Schottky analysis and these confirmed the results obtained by measuring the conductivity and capacitance. The carrier concentrations were higher in modified PEDOT:PSS sensors, confirming the improvement of the conductivity when implementing a secondary electrocatalytic material. Moreover, the flat band potentials were lower for PEDOT:PSS/AuNPS and PEDOT:PSS/ LUPC 2 than for PEDOT:PSS, again demonstrating the important effect of AuNPs and $\mathrm{LuPC}_{2}$ as doping agents.

Nyquist and Bode plots from EIS experiments confirmed that modified PEDOT:PSS sensors showed lower charge-transfer resistance (Rct), a reduction in the impedance module and also more negative values in the phase angle. These results are in accordance with those mentioned before and evidence the efficient electrocatality properties when combining PEDOT:PSS with $\mathrm{LuPC}_{2}$ or AuNPs in layered films.

The responses of the impedimetric electronic tongue for the red wine samples under study showed a Nyquist plot (Zimaginary vs Zreal) where the three wines elaborated with Tempranillo grapes had the highest values of Rct, whereas the other two wines, Tinta de Toro and the Tempranillo/Garnacha mixture, had lower values of Rct. This result might be correlated with the wine composition. A higher content of redox species, such as antioxidants, sugars or $\mathrm{SO}_{2}$, as well as a higher acidity and alcoholic degree, will provide higher values of Rct.

Further, the discrimination of red wines was performed by PCA following different strategies of data extraction methods. Firstly, we used a classical method of selecting resistance and capacitance data at a fixed frequency. However, using this method, the red wines were not discriminated in PCA analysis. Therefore, the feature extraction method was improved following two other ways: in the first, we selected parameters of the Nyquist and Bode plots obtained in EIS analysis in wines at a constant frequency (Zimaginary, Zreal and maximum phase angle) and, in 
the second, we obtained parameters from an equivalent electric circuit resulting from simulating the EIS results with different equivalent circuits in order to understand the electrochemical behavior of an electrode coated with a semiconductor polymer material. In the last method, the number of variables was increased to seven.

In the first improved feature extraction method, the results of the PCA analysis showed well-defined and separate clusters for each variety of wine. A 2D PCA score plot showed the wine elaborated with two different varieties (Tempranillo and Garnacha) clearly separated from the rest of the samples. In the second analysis, PCA analysis was also capable of discriminating between the wine samples; however, observing the 3D PCA score plot, we can conclude that the degree of discrimination was higher than when using variables from the Nyquist and Bode plots: red wines elaborated from Tempranillo grape and with similar chemical parameters appeared on the left side of the diagram, whereas wines elaborated with other varieties appeared separated from them and in between.

Finally, PLS-1 analysis was performed to obtain regression models able to predict chemical parameters in the wines from the data acquired by means of EIS experiments and from the equivalent circuit parameters. Both methods established good correlations with the chemical parameters provided by the oenological center, with high correlation coefficients and a low number of latent variables (in the range from 1 to 3 ). The values of the root mean square errors (RMSE) of calibration and prediction analysis were also low. However, in general, regression models obtained using the equivalent circuit parameters provided the best correlations with the chemical parameters, confirming that this method improves the quality of the feature extraction. 

Paper VII: Impedimetric electronic tongue based on nanocomposites for the analysis of red wines. Improving the variable selection method.

Sensors \& Actuators: B. Chemical 277 (2018) 365-372

\section{Impedimetric electronic tongue based on nanocomposites for the analysis of red wines. Improving the variable selection method \\ C. Garcia-Hernandez ${ }^{\text {a }}$, C. Salvo Comino ${ }^{\text {a }}$, F. Martín-Pedrosa ${ }^{\mathrm{b}}$, M.L. Rodriguez-Mendez ${ }^{\mathrm{a}}$, C. Garcia-Cabezon ${ }^{\mathrm{b}}$,}

a Grout UVaASens, Department of Inorganic Chemistry, Engineers School, Universidad de Valladolid, 47011, Valladolid, Spain

bepartment of Materials Science, Engineers School, Universidad de Valladolid, 47011, Valladolid, Spain

A R T I C L E I N F O

\section{Keywords:}

Impedimetric senso

Electronic tongue

Wine

\begin{abstract}
A B S T R A C T
An impedimetric electronic tongue to discriminate red wines with similar characteristics (same region, vintage and ageing method) is developed. The excellent performance achieved is due to the remarkable sensing properties of the nanocomposites forming the array and to the methodology developed to extract the input variables used in the statistical analysis. The multisensor system is formed by one PEDOT:PSS sensor and two nanocomposites formed by layers of PEDOT:PSS and gold nanoparticles (PEDOT:PSS/AuNP) or layers of PEDOT:PSS and lutetium bisphthalocyanine (PEDOT:PSS/LuPc 2 ). The well-known electrocatalytic activity of the sensing materials is promoted in the layered composites and enhances the cross-selectivity of the sensors. Besides, feature extraction method that uses an equivalent electric circuit model to obtain the input variables used in the statistical analysis has been developed. By using the statistical procedure of Principal Component Analysis, the improved electronic tongue is able to discriminate three wines from the variety Tempranillo, one wine of the variety Tinta de Toro (a clon of Tempranillo) and one wine elaborated with a coupage of $90 \%$ Tempranillo and $10 \%$ Garnacha variety. The combination of layered nanomaterials and the new feature extraction method also enhances the capability of the impedimetric electronic tongue to predict chemical variables using Partial Leas Squares regression.
\end{abstract}

\section{Introduction}

Electronic tongues (e-tongue) are an excellent alternative to traditional methods of analysis for the control of products and processes in the agrifood sector. Through a system of multisensors combined with chemometric tools, these systems are able to classify samples and quantify their physicochemical parameters $[1,2]$. They have many advantages over traditional analysis techniques. The main one is that the measures suppose an important saving of time because they are no individual tests, do not require preprocessing of the sample or specialized technicians. They are therefore an excellent tool in the quality control of food, especially in situations where only qualitative or semiquantitative information is required $[3,4]$.

There are many types of e-tongues depending on the variety of sensitive materials and transduction methods [5]. Most of the works in the field make use of sensors based on electrochemical techniques in cluding potentiometric, voltammetric or amperometric sensors [6-9]. In contrast, e-tongues based on impedimetric sensors have been studied in a much lesser extent.
E-tongues using impedance spectroscopy (IS) technique were initially developed by Riul et al. [10]. They are based on interdigitated electrodes modified with different chemosensitive materials deposited by LB (Langmuir-Blodgett) or LBL (Layer by Layer) techniques [11]. These etongues are based on the application of a sinusoidal signal in a range of frequencies with a constant signal amplitude and the measurement of the impedance at a fixed frequency [12,13]. For instance, applying sinusoidal signals (from $20 \mathrm{~Hz}$ to $100 \mathrm{kHz}$ ) to interdigitated electrodes coated with different polymeric materials, it was possible to detect and discriminate sucrose, sodium chloride, potassium chloride and hydrochloric acid [11]. Quantification of ions (sodium, potassium and ammonium) in solution could also be achieved under static conditions [14] or under a continuous flow system [15]. Pioggia et al. successfully developed a polymer membrane electrode system to measure standard solutions that simulated the five basic flavors (sweetness, sourness, bitterness, saltiness and umami or savory taste) $[16,17]$. Masot developed an electrochemical impedimetric spectroscopy (EIS) e-tongue to determine physicochemical parameters such as salt and moisture content in meats [18], to detect the level of curing in cheeses [19], to study the freshness of fish [20], to

\footnotetext{
* Corresponding author.

E-mail addresses: mluz@eii.uva.es (M.L. Rodriguez-Mendez), anacrigar@gmail.com (C. Garcia-Cabezon).
}

https://doi.org/10.1016/j.snb.2018.09.023

Received 12 April 2018; Received in revised form 3 September 2018; Accepted 6 September 2018

Available online 07 September 2018

0925-4005/ (c) 2018 Elsevier B.V. All rights reserved. 
Paper VII: Impedimetric electronic tongue based on nanocomposites for the analysis of red wines. Improving the variable selection method.

determine the botanical origin of honey [21] or to detect contaminants of microbiological origin [22]. The impedimetric e-tongues have also been used for the recognition of beverages as mineral waters $[23,24]$ or black teas [25]. There are few works using impedimetric e-tongues applied to discrimination of wines $[13,26]$. In fact, most of the works in the oenological field use potentiometric [27,28] or voltammetric [29-32] sensors.

Many efforts have been made to improve the performance of etongues. In many cases such works were related to the use of new sensing materials that could improve the sensitivity and selectivity of the sensors forming the array. Phtalocyanines has been successfully used in LBL films for dopamine detection using EIS [33]. Moreover, it has been demonstrated that the combination of electrocatalytic materials can produce synergistic effects improving the performance of voltammetric sensors in the detection of phenols of interest in the food industry [34].An example of this in EIS sensors has been published on pesticide detection in real samples using nanocomposites modified with graphene and gold nanoparticles using the electrical resistance response in the medium frequency region [35].

The objective of this work was to develop an improved impedimetric e-tongue for the analysis of red wines. For this purpose, two strategies were followed. On one hand, to develop new impedimetric sensors based on combinations of well-known electrocatalytic materials including PEDOT:PSS [34,36,37], gold nanoparticles [38] and phthalocyanines [39]. On the other hand, the impedance spectroscopy measurements used for impedimetric e-tongues are diverse, in some cases measurements of electrical resistance and/or capacitance have been used at a fixed frequency value $[26,35,40]$. In this work, different possibilities were tested, including the fitting by means of an equivalent circuit, in order to determine which method will offer the best discrimination capability.

The performance of the improved system will be tested by evaluating its capability to discriminate red wines with similar characteristics but with different chemical composition and to establish correlations between the results obtained with the e-tongue and the traditional chemical analysis including Total Polyphenol Index (TPI), Folin Index, Total acidity, $\mathrm{SO}_{2}$ content, sugars content, alcoholic degree and $\mathrm{pH}$.

\section{Materials and methods}

\subsection{Reagents and solutions}

PEDOT:PSS aqueous solution (3.0-4.0\% in $\mathrm{H}_{2} \mathrm{O}$, high conductivity grade) was purchased from Sigma-Aldrich. Gold nanoparticles (AuNPs, $30-40 \mathrm{~nm}$ ) were synthesized according to a modification of the procedure proposed by Slot and Geuze [41,42]. Using this procedure, a red colloid with a UV absorbance maximum at $\lambda=540 \mathrm{~nm}$ was obtained. The lutetium(III) bisphthalocyaninate $\left(\mathrm{LuPc}_{2}, 0.05 \mathrm{~g} \mathrm{~L}^{-1}\right)$ was synthesized using a method developed by our group [43].

\subsection{Preparation of the electrochemical sensors and biosensors}

Sensors were prepared using a spin coater model 1H-D7 (Micasa Co., Tokyo, Japan). ITO glass substrates $\left(1 \mathrm{~cm}^{2}\right.$ of surface) were used as the substrate. Prior to the film deposition, substrates were washed in an ultrasonic bath with acetone and rinsed twice with deionized water (MilliQ).

PEDOT:PSS was diluted 1:10 in deionized water and stirred in an ultrasonic bath for $10 \mathrm{~min}$. Then, $100 \mu \mathrm{L}$ of the solution was dropped onto the ITO glass (Indium Tin Oxide glass) and spin-coated with a ramp of $120 \mathrm{~s}$ from low speed to $2000 \mathrm{rpm}$ and then, it was stayed during $120 \mathrm{~s}$. The PEDOT:PSS films thus obtained were annealed at $150^{\circ} \mathrm{C}$ for $15 \mathrm{~min}$.

Electrochemical sensors based on layered composites were formed by alternate layers of PEDOT:PSS and a secondary electrocatalytic material (EM) (PEDOT:PSS/EM). They were prepared on the surface of PEDOT:PSS films by spin-coating $200 \mu \mathrm{L}$ of AuNPs or $100 \mu \mathrm{L}$ of $\mathrm{LuPc}_{2}$, at
$2000 \mathrm{rpm}$ for $120 \mathrm{~s}$ (ramp of $120 \mathrm{~s}$ ) followed by annealing at $150^{\circ} \mathrm{C}$ for 15 min to obtain PEDOT:PSS/LuPc 2 and PEDOT:PSS/AuNPs sensors, respectively.

\subsection{Characterization of the sensors}

A SEM-FEI (QUANTA 200 F) (Hillsboro, USA) was used to record the images of the electrode surfaces. Square resistance was measured using a four-point tester (HAAMEG HM 8040-2).

Voltammetric measurements were obtained using a potentiostat/ galvanostat PGSTAT128 (Autolab Metrohm, Utrecht, The Netherlands). The electrochemical cell was a three- electrode system using the corresponding PEDOT:PSS or PEDOT:PSS/EM modified electrode as the working electrode, a platinum foil with a size of $2 \mathrm{~cm} \times 1 \mathrm{~cm}$ as the counter electrode and an $\mathrm{Ag} / \mathrm{AgCl} / \mathrm{KCl} 3 \mathrm{M}$ electrode as the reference electrode.

Mott-Shottky analysis and EIS experiments were performed using a SOLARTRON Impedance Analyzer, SI 1260, (Hampshire, England). The Mott-Shottky measurements were performed at a fixed frequency of $100 \mathrm{~Hz}$ in $\mathrm{KCl}\left(0.1 \mathrm{~mol} \mathrm{~L}^{-1}\right)$. The impedance spectroscopy was carried out by applying a signal amplitude of $10 \mathrm{mV}$, with frequencies that varied logarithmically from $0.1 \mathrm{~Hz}$ to $10 \mathrm{kHz}$ at different working potentials. Measurements were made in three electrolytes: $\mathrm{KCl}$ $\left(0.1 \mathrm{~mol} \mathrm{~L}^{-1}\right),\left[\mathrm{Fe}(\mathrm{CN})_{6}\right]^{4-3 \cdot 3}\left(5 \mathrm{mmol} \mathrm{L}{ }^{-1}\right)$ in $\mathrm{KCl}\left(0.1 \mathrm{~mol} \cdot \mathrm{L}^{-1}\right)$ and diluted wines $1: 1$ in $\left.\mathrm{KCl}\left(0.1 \mathrm{~mol} \mathrm{~L}^{-1}\right)\right)$. Impedance spectra were fitted with aid of the Zview2 software, Scribner Associates, Inc. Four replicas per sample were carried out.

\subsection{Wine samples}

Wines were elaborated purposely for this work in the "Estación Enológica de Rueda" located in Castilla y León area (Valladolid, Spain). They included five red wines elaborated from grapes harvested in five cellars of the same Denomination of Origin (DO). They were vinified using the same method and aged for 6 months in oak barrel and 18 months in bottle. From the five wines, three of them (W3, W4 and W5) were prepared form the same variety of grape (Tempranillo). W1 was elaborated using grapes of the variety Tinta de Toro which is a clon of the variety Tempranillo and W2 was elaborated using a mixture of Tempranillo (90\%) and Garnacha (10\%) grapes. Total Polyphenol Index (TPI), Folin Index, Total acidity, $\mathrm{SO}_{2}$ content, sugars content, alcoholic degree and $\mathrm{pH}$ were analyzed following international regulations [44]. Results of the chemical analysis are collected in Table 1.

\subsection{Chemometrics}

EIS measurements were normalized using Matlab v2014b (The Mathworks Inc., Natick, MA, USA). A non-supervised multivariate method, Principal Component Analysis (PCA) was used to evaluate the discrimination capability of the impedimetric e-tongue. The impedance curves used as data source for statistical analysis were first pre-processed. Then, they were adjusted to equivalent circuit using Zview2 software. Partial Least Square Regression-1 (PLS-1) was used to establish correlations between the results obtained from the impedimetric etongue and the chemical parameters.

\section{Results and discussion}

\subsection{Structural characterization}

The surface structure of PEDOT:PSS, PEDOT:PSS/LuPc ${ }_{2}$ and PEDOT:PSS/AuNPs sensors was examined using SEM. PEDOT:PSS films showed a homogeneous smooth surface (Fig. 1a). The structure was not altered upon modification with $\mathrm{LuPc}_{2}$ (Fig. 1b). The surface of the PEDOT:PSS/AuNPs showed the expected smooth surface of the PEDOT:PSS films where nanoparticles were well-distributed on the film 
Chapter 3: Results and discussion

Paper VII: Impedimetric electronic tongue based on nanocomposites for the analysis of red wines. Improving the variable selection method.

Table 1

Average chemical parameters measured in wines.

\begin{tabular}{|c|c|c|c|c|c|}
\hline WINE & W1 & W2 & w3 & W4 & w5 \\
\hline D.O. (Origin) & Toro & Cigales & Ribera Duero & Ribera Duero & Ribera Duero \\
\hline Variety & Tinta Toro & Tempranillo $(90 \%)$ Garnacha(10\%) & Tempranillo & Tempranillo & Tempranillo \\
\hline Year & 2014 & 2014 & 2014 & 2014 & 2014 \\
\hline Folin & 0.475 & 0.650 & 0.651 & 0.658 & 0.720 \\
\hline TPI & 0.684 & 0.727 & 0.618 & 0.715 & 0.720 \\
\hline Total Acidity $(\mathrm{g} / \mathrm{1})$ & 4.94 & 4.83 & 4.69 & 4.64 & 4.54 \\
\hline $\mathrm{SO}_{2}(\mathrm{mg} / \mathrm{l})$ & 77 & 49 & 92 & 89 & 97 \\
\hline Reducing Sugar $(g / 1)$ & 1.2 & 1.3 & 1.6 & 1.4 & 1.4 \\
\hline Glucose + Fructose $(g / 1)$ & 0.56 & 0.11 & 0.35 & 0.22 & 0.19 \\
\hline Alcoholic Degree & 14.35 & 13.97 & 14.78 & 14.41 & 14.50 \\
\hline $\mathrm{pH}$ & 3.78 & 3.79 & 3.79 & 3.74 & 3.78 \\
\hline
\end{tabular}

surface and only few nanoparticle clusters were observed, (Figure 1c and $1 \mathrm{~d})$.

\subsection{Conductivity and capacitance measurements}

The conductivity of the electrodes was measured using the fourpoint probe test. The conductivity of PEDOT:PSS $\left(3.6 \times 10^{4} \Omega^{-1} \mathrm{~m}^{-1}\right)$ increased when films of lutetium phthalocyanine (PEDOT:PSS $/ \mathrm{LuPc}_{2}$ : $6.4 \times 10^{4} \Omega^{-1} \mathrm{~m}^{-1}$ ) or AuNPs (PEDOT:PSS/AuNPs: $5.1 \times 10^{4} \Omega^{-1} \mathrm{~m}^{-1}$ ) were deposited on the top of the PEDOT:PSS film. This enhancement was similar to the increase observed when dopants were added to an aqueous solution of PEDOT:PSS [45]. The intensification of the conductivity in the PEDOT:PSS/LuPc ${ }_{2}$ film can be associated with the increase in charge carriers caused by the interaction between PEDOT chains and the charge carriers of the intrinsic semiconductor $\mathrm{LuPc}_{2}$
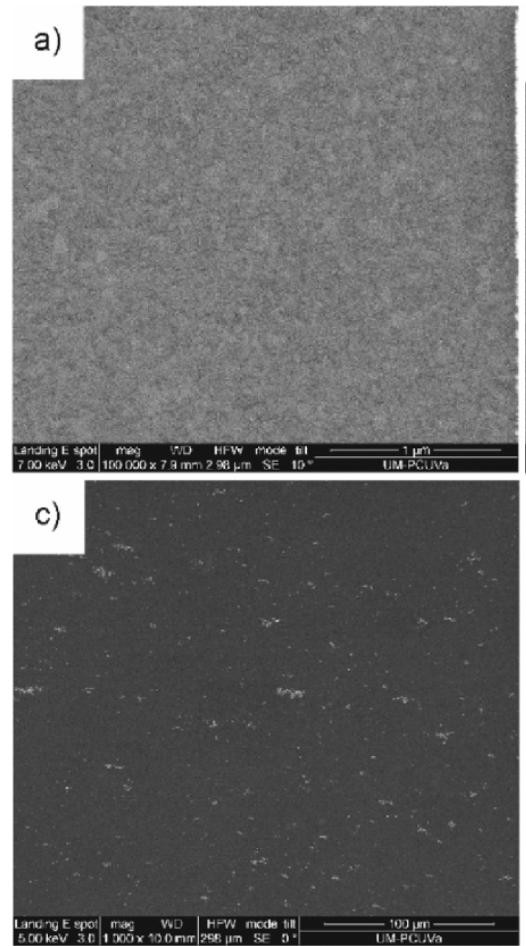

Fig. 1. SEM images of ITO electrodes modified with (a) PEDOT:PSS, (b) PEDOT:PSS/LuPc ${ }_{2}$, (c) PEDOT:PSS/AuNPs, and (d) PEDOT:PSS/AuNPs at a higher magnification.

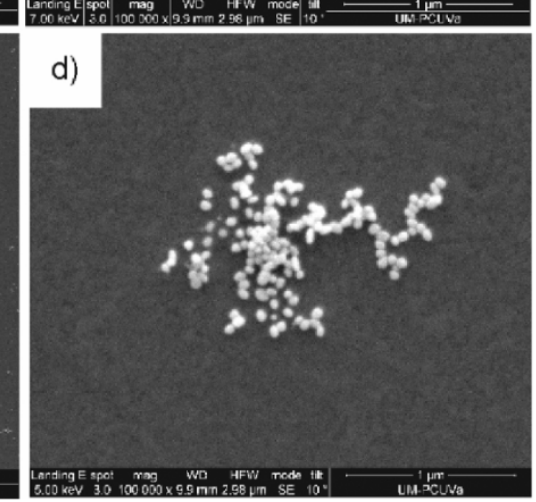

[46]. The increase in PEDOT:PSS/AuNPs can be correlated with the enlargement of the effective surface area caused by the AuNPs and to the existence of mixed valence states on their surface.

In order to further investigate the effect of the secondary electrocatalytic materials deposited on PEDOT:PSS films, capacitance versus voltage $(\mathrm{C}-\mathrm{V})$ measurements were performed from $-0.5 \mathrm{~V}$ to $0.5 \mathrm{~V}$. The capacitance $\mathrm{C}$ was calculated by using the relation $\mathrm{C}=(-\mathrm{Z} \text { '2 } 2 \pi \mathrm{w})^{-1}$ where $Z$ " is the imaginary part of the impedance and $w$ is de frequency. As shown in Fig. 2a, at low potentials C slightly decreased and suddenly raised at intermediate potentials. This behavior is similar to that observed in ITO/PEDOT:PSS photoelectrochemical cells and is characteristic for p-doped systems [47].

With the aim of establishing the number of charge carriers, the Mott-Schottky equation (Eq. (1)) was applied [48].

b)

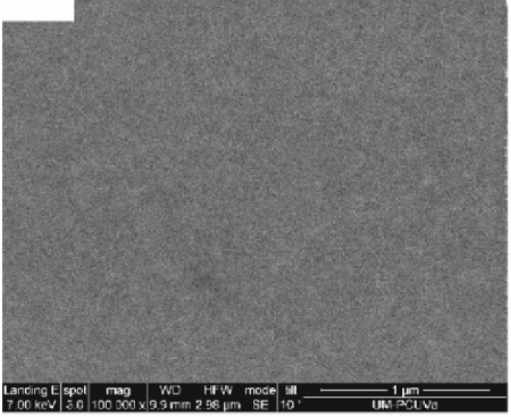

d) 
Paper VII: Impedimetric electronic tongue based on nanocomposites for the analysis of red wines. Improving the variable selection method.
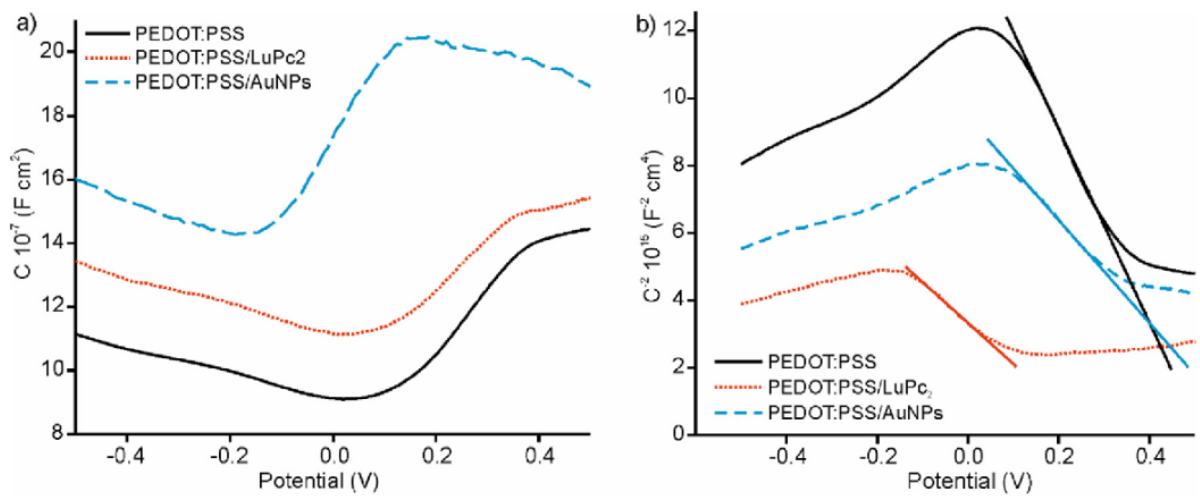

Fig. 2. (a) Capacitance-voltage response and (b) Mott-Schottky analysis.

$\frac{1}{C^{2}}=\frac{2}{\varepsilon \varepsilon_{0} e N_{D}}\left(V-V_{F B}-\frac{K T}{e}\right)$

(1)

Where, $C$ is capacitance, $N_{D}$ is the donor/acceptor density, $\varepsilon$ is the dielectric constant of the nanocomposite, $\varepsilon_{0}$ is the dielectric constant under vacuum, $\mathrm{e}$ is the electron charge, $\mathrm{V}_{\mathrm{FB}}$ is the flat band potential, $\mathrm{T}$ is the absolute temperature and $\mathrm{K}$ is the Boltzmann constant.

Mott-Schottky plots were obtained using a voltage sweep from -0.5 to $0.5 \mathrm{~V}$ and an input signal amplitude of $50 \mathrm{mV}$ peak to peak at $100 \mathrm{~Hz}$ in $0.1 \mathrm{mol. \textrm {L } ^ { - 1 }} \mathrm{KCl}$ (Fig. 2b). Two important parameters were read directly from $1 / \mathrm{C}^{2}-\mathrm{V}$ plots: $\mathrm{V}_{\mathrm{FB}}$ which is defined by the intercept of the straight line with the potential axis and $N_{D}$ that can be determined from the slope of the Mott-Schottky plot. As observed in the Fig. 2b, $\mathrm{V}_{\mathrm{FB}}$ was lower for PEDOT:PSS/AuNPs nanocomposite confirming the important effect of AuNPs as doping agents. The dopant density $\mathrm{N}_{\mathrm{D}}$ was clearly higher in layered nanocomposites $\left(0.9810^{15} \mathrm{~cm}^{-3}\right.$ for PEDOT:PSS, 2.9 $10^{15} \mathrm{~cm}^{-3}$ for PEDOT:PSS/LuPc 2 and $3.110^{15} \mathrm{~cm}^{-3}$ for PEDOT:PSS/ AuNPs) and this suggests an increase in the surface charge density which is in good correlation with the improvement of conductivity observed for PEDOT:PSS/AuNPs and PEDOT:PSS/ $\mathrm{LuPc}_{2}$. The higher conductivity and the higher surface charge density could induce an improvement on electrocatalytic properties. This will be analyzed in the next section.

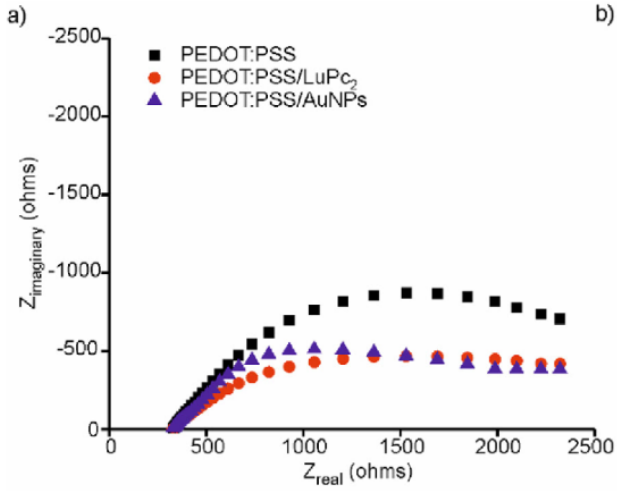

3.3. EIS characterization

The interfacial properties of the layered composites PEDOT:PSS/EM electrodes were investigated by EIS using $\left[\mathrm{Fe}(\mathrm{CN})_{6}\right]^{4-/ 3-}$ as a redox probe. The impedance measurements were performed at various potentials $(-1 \mathrm{~V},-0.5 \mathrm{~V}, 0 \mathrm{~V}, 0.5 \mathrm{~V}$ and $1 \mathrm{~V})$.

The Nyquist plots ( $\mathrm{Z}_{\mathrm{REAL}}$ vs. $\mathrm{Z}_{\mathrm{IMAGINARY}}$ ) registered at $-1 \mathrm{~V},-0.5 \mathrm{~V}$, $0 \mathrm{~V}, 0.5 \mathrm{~V}$ and $1 \mathrm{~V}$ showed a semicircular shape in the high-frequency region. This region corresponds to electron-transfer limited processes and the diameter of the semicircle is equivalent to the electron transfer resistance $\left(\mathbf{R}_{\mathrm{ct}}\right)$. In experiments performed at $-1 \mathrm{~V},-0.5 \mathrm{~V}, 0 \mathrm{~V}, 0.5 \mathrm{~V}$ and $1 \mathrm{~V}$, it was observed that the diameter changed with the applied potential (Fig. 3.a shows the responses at 0.5 V. Figure S1 illustrates the curves obtained at $0 \mathrm{~V}$. For all three sensors, the smallest $\mathbf{R}_{\mathrm{ct}}$ values were obtained at $0.5 \mathrm{~V}$, near to the oxidation peak of the

$\left[\mathrm{Fe}(\mathrm{CN})_{6}\right]^{4-/ 3-}$ couple. Layered composites showed lower $\mathbf{R}_{\mathrm{ct}}$ values than PEDOT:PSS indicating that charge transfer rates were higher due to the efficient electrocatalytic properties of the nanocomposites. Bode diagrams (Fig. 3b), showed that the impedance module was also considerably reduced and the phase angle reached less negative values for layered composites. This agrees well with the aforementioned conductivity measurements. The smaller $\mathbf{R}_{\mathrm{ct}}$ values revealed a low
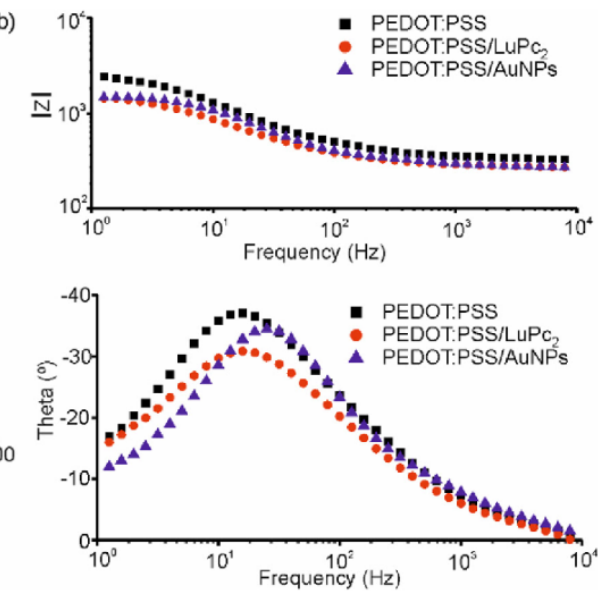

Fig. 3. (a) Nyquist plots and (b) Bode plots recorded in $0.1 \mathrm{~mol} \mathrm{~L}{ }^{1} \mathrm{KCl}$ containing $5 \mathrm{mmol} \mathrm{L}^{-1}\left[\mathrm{Fe}(\mathrm{CN})_{6}\right]^{4 / 3 \cdot}$ registered at $0.5 \mathrm{~V}$. 


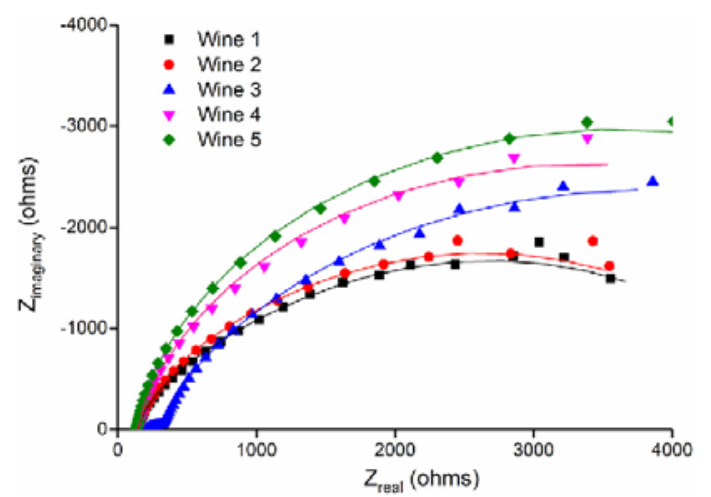

Fig. 4. EIS spectra of PEDOT:PSS/AuNPs electrode obtained for differen varieties of wines from $10^{4}$ to $0.1 \mathrm{~Hz}$ registered at $-0.5 \mathrm{~V}$. Equivalent circuit modeling are also included (solid lines).

electron-transfer resistance which might be caused by the enhanced electrocatalytic effect caused by the interaction between PEDOT:PSS and the AuNPs and $\mathrm{LuPc}_{2}$ deposited films. The improvement of the observed performance can be attributed to the enhanced electron transfer rate provided by the composites due to the interactions between two electrocatalytic components.

\subsection{Electronic tongue dedicated to wines}

\subsubsection{The response of the array of sensors to wines}

The sensors responses to red wines are illustrated in Fig. 4. Nyquist plots registered at different potentials $(1 \mathrm{~V},-0.5 \mathrm{~V}, 0 \mathrm{~V}, 0.5 \mathrm{~V}$ and $1 \mathrm{~V})$ were characterized by an incomplete semicircle. In the wine matrix, the lowest $R_{\mathrm{ct}}$ values were always obtained at $-0.5 \mathrm{~V}$ (Figure S2 shows the data of the three sensors at $0 \mathrm{~V}$ and at $-0.5 \mathrm{~V}$ for the wine W2). Fig. 4 shows the impedimetric responses (at $-0.5 \mathrm{~V}$ ) of the PEDOT:PSS/ AuNPs sensor immersed in red wines. It was observed that the three wines elaborated with Tempranillo grapes (W3, W4 and W5) showed the highest $R_{c t}$ values. Wines $W 1$ and $W 2$ elaborated with different grapes showed lowest $R_{\mathrm{ct}}$ values. This trend can be correlated to the higher content of W3, W4 and W5 in redox species (antioxidants measured by Folin, sugars and $\mathrm{SO}_{2}$ ) and their higher acidity and alcoholic degree.

Therefore a higher $R_{c t}$ is related with a higher content of redox species. To explain this two issues must be considered. First, wine is a complex mixture. Second, there is not an inverse correlation between concentration and $\mathrm{R}_{\mathrm{ct}}$ as it would be intuitively thought. In some studies, a higher $\mathbf{R}_{\mathrm{et}}$ was correlated with a lower content of glucose [49] or cathecol [50], while on others works the opposite tendency was observed, a higher $\mathbf{R}_{\mathrm{ct}}$ for higher concentration of redox species (chorpyrifos [51], cathecol [52], urea [53], thrombin [54], etc) were observed. Therefore, the correlation is a function of the interaction between the sensor and the analyte.

Another important observation was that the semicircle diameter increased along the sequence PEDOT:PSS/AuNPs < PEDOT:PSS/ $\mathrm{LuPC}_{2}<$ PEDOT:PSS. The small $\mathbf{R}_{\mathrm{ct}}$ values revealed a low electrontransfer resistance which might be caused by the synergetic enhanced effect between PEDOT:PSS and the secondary electrocatalytic materials. Moreover, this result indicates that sensors show different sensitivity and can be used as the sensing units of an impedimetric electronic tongue.

3.4.2. Impedimetric electronic tongue dedicated to wines. Improvement of the discrimination capability by developing new feature extraction methods

The outputs of the array of sensors were treated by chemometric techniques. In order to improve the quality of the feature extraction, impedance curves were pre-processed following different methods. First, we used the classical method that selects resistance/capacitance data at a fixed frequency $[26,35]$. Using this feature extraction method, the PCA analysis could not discriminate the similar wine samples (Supplementary Figure S3). Therefore, this method could be applied to interdigitated electrodes by itself or coated with several chemosentitive materials but it is not recommended when these materials are deposited on ITO. The feature extraction method was improved following two other ways: (1) Selection of other parameters of the impedance spectrum and (2) obtain parameters from an equivalent electric circuit.

In the method (1), the impedance parameters selected were: the maximum phase angle $\left(\theta_{\mathrm{MAX}}\right)$ obtained from the Bode diagram and the real ( $\mathrm{Z}_{\mathrm{REAL}}$ ) and the imaginary ( $\mathrm{Z}_{\text {MagGrary }}$ ) impedance data at $1.25 \mathrm{~Hz}$ taken from the Nyquist diagram. This frequency was selected because for frequencies lower than $1 \mathrm{~Hz}$, an oscillation of the impedance (real and imaginary) that could be related to the uptake-desorption of chemical species on the electrode was observed, (Supplementary Figure S4). The value of the impedance module was discarded since it was very similar for all wine samples at any frequency value.

In method (2), different strategy to improve the quality of the variable extraction, an equivalent electric circuit was established. After several attempts to simulate the EIS results with different equivalent circuits, the quality of the fittings and the possibility assigning a physical meaning to the circuit components led to simulate the electrochemical behavior of an electrode coated with a semiconductor polymer material [51] (Fig. 5).

In the proposed model, the high frequency region, $10 \mathrm{kHz}-100 \mathrm{~Hz}$, is dominated by the resistance of the electrolyte $\left(\mathbf{R}_{\mathrm{s}}\right)$. In the mid-frequency region, $100 \mathrm{~Hz}-0.1 \mathrm{~Hz}$, the process is related to the formation of an electrical double layer at the film/solution interface. The charge transfer resistance $\left(\mathbf{R}_{\mathrm{ct}}\right)$ is related with two effects: the energy potential associated with oxidation/reduction processes and with the energy barrier of the redox species reaching the electrode. The whole interface impedance has been modelled by a constant phase element (CPE) (which represents a non-ideal capacitive response of the interface material-solution), the depressedcircle is an indication of this non-pure capacitive behavior.

The impedance of a CPE element is defined as:

$$
Z_{C P E}=\frac{1}{C(j \omega)^{n}}
$$

where $\mathrm{C}$ is a parameter numerically equal to the admittance at $\omega=1 \mathrm{rad} \mathrm{s}^{-1}, j$ is the imaginary unit, $\omega$ is the frequency and $\mathrm{n}$ is a number that moves between 0 and 1 . Finally, at low frequency region, the Warburg impedance $(W)$ represents the delay arising from diffusion of the electroactive species to the electrode.

The Generalized Finite Warburg is defined by:

$Z_{\text {吸 }}=\frac{W_{R} \tanh \left[\left(j \omega W_{C}\right)^{p / h}\right]}{\left(j \omega W_{C}\right)^{W / n}}$

where $W_{R}, W_{N}$ and $W_{C}$ are the Warburg parameters. As an example, Table 2 shows the average values obtained with this fitting for the three sensors in wine $\mathrm{W} 2$.

The results of the PCA analysis performed with the parameters obtained from the impedance curves $\left(\mathrm{Z}_{\mathrm{REAL}}\right.$ and $\mathrm{Z}_{\mathrm{DMAGNARY}}$ at $1.25 \mathrm{~Hz}$ and $\left.\theta_{\text {MAX }}\right)$ are shown in Fig. 6. PCA scores plots showed well-defined and separated clusters for each variety of wine. The captured information was $97 \% \quad(\mathrm{PC} 1=63 \% ; \quad \mathrm{PC} 2=25 \% ; \quad \mathrm{PC} 3=9 \%)$. Clusters were

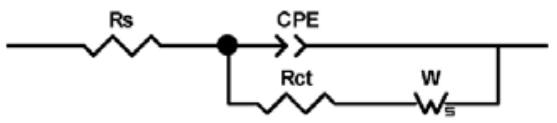

Fig. 5. Equivalent electric circuit to interpret EIS measurements. 
Paper VII: Impedimetric electronic tongue based on nanocomposites for the analysis of red wines. Improving the variable selection method.

Table 2

EIS equivalent circuit parameters measured in wine $\mathrm{W} 2$ at $-0.5 \mathrm{~V}$.

\begin{tabular}{|c|c|c|c|c|c|c|c|c|}
\hline Sensors & $\begin{array}{l}R_{S} \\
\left(\Omega \mathrm{cm}^{2}\right)\end{array}$ & $\begin{array}{l}\mathrm{CPE}_{\mathrm{C}} \\
\left(\mathrm{s}^{\mathrm{n}} \Omega^{-1} \mathrm{~cm}^{-2}\right)\end{array}$ & $\mathrm{CPE}_{\mathrm{n}}$ & $\begin{array}{l}\mathrm{R}_{\mathrm{ct}} \\
\left(\Omega \mathrm{cm}^{2}\right)\end{array}$ & $\begin{array}{l}W_{R} \\
\left(\Omega \mathrm{cm}^{2}\right)\end{array}$ & $w_{n}$ & $\begin{array}{l}W_{C} \\
\left(\mathrm{~s}^{\mathrm{n}} \Omega^{-1} \mathrm{~cm}^{-2}\right)\end{array}$ & $\begin{array}{l}\chi^{2} \\
\left(10^{-5}\right)\end{array}$ \\
\hline PEDOT-PSS & 159.3 & $2.7410^{-5}$ & 0.89 & 4951 & 4646 & 0.55 & 0.55 & 6.4 \\
\hline PEDOT-PSS $/ \mathrm{LuPc}_{2}$ & 159.7 & $2.7310^{-5}$ & 0.88 & 6301 & 570.5 & 0.73 & 0.53 & 9.9 \\
\hline PEDOT-PSS/AuNPs & 150.5 & $2.9110^{-5}$ & 0.84 & 2151 & 2691.6 & 0.46 & 0.56 & 5.9 \\
\hline
\end{tabular}

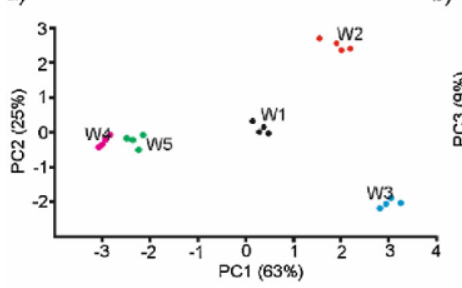

b)

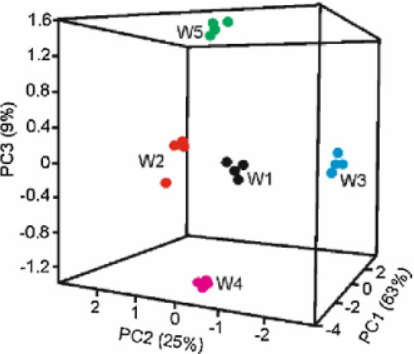

C) - PEDOT:PSS

- PEDOT:PSS/LuPc

^PEDOT:PSS/AUNPS

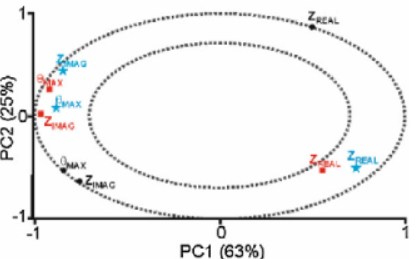

Fig. 6. PCA corresponding to the classification of the wines (a) 2D scores plots, (b) 3D scores plots, and (c) correlation loadings plot using $\mathrm{Z}_{\mathrm{REAL}}$ and $\mathrm{Z}_{\mathrm{IMAGINARY}}$ and $\theta_{\text {MAX }}$ obtained for PEDOT:PSS, PEDOT:PSS/LuPc ${ }_{2}$ and PEDOT:PSS/AuNP.

distributed according to the variety of grape and the chemical composition. The loadings provided by each sensor, Fig. $6 \mathrm{c}$, appeared in different regions of the diagram confirming that the secondary electrocatalytic materials played an important role in the selectivity of the sensors.

A different PCA analysis was carried out based on the parameters obtained from the fitting to an equivalent electric circuit showed in Fig. 5. Fig. 7 shows the final PCA analysis and the correlation loadings plots.

In the case of PCA using equivalent circuit parameters, a good separation of the clusters was also obtained. Due to the increase in the numbers of variables the three principal components bring an important amount of information. The PC1, PC2 and PC3 explain the $60 \%$ $25 \%$ and $6 \%$ of the information, respectively.

Multivariate inspection of impedimetric data using both methods revealed the high capability of discrimination of this impedimetric etongue. It could be applied to the recognition of wines showing excellent sensitivity similar to the obtained with our voltammetric etongues $[29,32]$ comprised of voltammetric electrodes chemically modified with different sensitive materials. Moreover, there is no requirement of active species in the measuring system and no need of a standard reference electrode. Although both feature extraction systems provide noticeable information about the samples, a better correlation with the composition was obtained using the equivalent circuit parameters. In the 3D PCA it could be observed that samples were located according to their variety and their composition. Wines W3, W4 and W5 elaborated with the variety Tempranillo and with similar Folin, sugar content and acidity appear on the left side of the diagram, whereas wines elaborated with other varieties, W1 and W2 with lower Folin, sugar content, sugar and acidity appeared on the right side.

\subsubsection{Correlations with chemical parameter:}

By means of regression techniques, the results obtained using the impedimetric e-tongue can be correlated with the chemical composition of the samples. The statistical parameters obtained from the Partial Least Squares (PLS) regression models, correlating the impedance spectrum parameters $\left(\theta_{\text {MAX }}, Z_{R E A L}, Z_{\text {IMAGYNARY }}\right.$ ) or the equivalent circuit parameters $\left(\mathrm{CPE}_{\mathrm{C}}, \mathrm{CPE}_{\mathrm{n}}, \mathrm{R}_{\mathrm{cv}}, \mathrm{W}_{\mathrm{R}}, \mathrm{W}_{\mathrm{C}}, \mathrm{W}_{\mathrm{n}}\right)$ with chemical parameters are shown in Tables 3 and 4 respectively. Using both methods, the squared correlation coefficients $\left(\mathbf{R}^{2}\right)$ obtained for calibration and prediction were high and the number of latent variables (LV) was low. The values of the root mean square error (RMSE) of calibration/prediction which are an estimate of the absolute error were also low.

The regression using the equivalent circuit parameters provided the best correlations and lower residual errors with a lower number of latent variables, confirming that this method improves the quality of the features extraction.

The prediction ability of the polyphenol index, alcoholic degree and

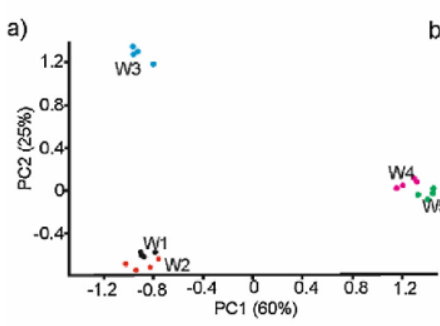

b)

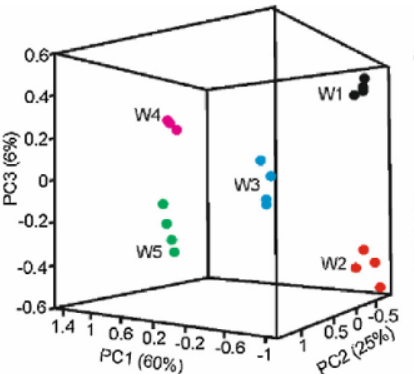

- PEDOT:PSS *PEDOT:PSS/AUNPS

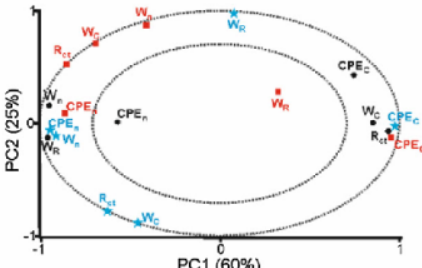

Fig. 7. PCA corresponding to the classification of the wines using equivalent circuit parameters. (a) 2D scores plot, (b) 3D scores plot, and (c) correlation loadings plot obtained for PEDOT:PSS, PEDOT:PSS/LuPc 2 and PEDOT:PSS/AuNP. 
Paper VII: Impedimetric electronic tongue based on nanocomposites for the analysis of red wines. Improving the variable selection method.

Table 3

Results of the PLS-1 analysis using impedance spectrum parameters.

\begin{tabular}{llllll}
\hline Parameters & $\mathrm{R}_{\mathrm{c}}^{21}$ & $\mathrm{RMSE}_{\mathrm{c}}{ }^{2}$ & $\mathrm{R}_{\mathrm{p}}^{23}$ & $\mathrm{RMSE}_{\mathrm{p}}{ }^{4}$ & $\mathrm{LV}$ \\
\hline FOLIN & 0.8288 & 0.0336 & 0.8219 & 0.0361 & 3 \\
TPI & 0.9554 & 0.0139 & 0.9381 & 0.0173 & 3 \\
Acidity & 0.9815 & 0.0231 & 0.9815 & 0.0263 & 3 \\
SO $_{2}$ & 0.9919 & 1.5478 & 0.9876 & 2.0110 & 2 \\
Reducig Sugars & 0.9420 & 0.0430 & 0.9666 & 0.0575 & 3 \\
Glucose + Fructose & 0.8902 & 0.0430 & 0.8308 & 0.0681 & 3 \\
PAlcoholic & 0.9815 & 0.0355 & 0.9763 & 0.0422 & 2 \\
pH & 0.8288 & 0.0336 & 0.8219 & 0.0361 & 3 \\
\hline
\end{tabular}

LV latent variables.

1 Squared correlation coefficient in calibration.

${ }^{2}$ Root mean square error of calibration.

${ }^{3}$ Squared correlation coefficient in prediction.

4 Root mean square error of prediction.

Table 4

Results of the PLS-1 analysis using equivalent circuit parameters.

\begin{tabular}{llllll}
\hline Parameters & $\mathrm{R}_{\mathrm{C}}^{21}$ & $\mathrm{RMSE}_{\mathrm{C}}{ }^{2}$ & $\mathrm{R}_{\mathrm{p}}^{23}$ & $\mathrm{RMSE}_{\mathrm{p}}{ }^{4}$ & $\mathrm{LV}$ \\
\hline FOLIN & 0.9913 & 0.0593 & 0.9886 & 0.0674 & 2 \\
TPI & 0.9974 & 0.0363 & 0.9965 & 0.0423 & 1 \\
Acidity & 0.9977 & 0.2247 & 0.9963 & 0.2857 & 2 \\
$\mathrm{SO}_{2}$ & 0.9894 & $\mathbf{8 . 4 4 8 0}$ & 0.9859 & 9.7800 & 2 \\
Reducig Sugar & 0.9894 & 0.1549 & 0.9858 & 0.1794 & 2 \\
Glucose + Fructose & 0.9837 & 0.0415 & 0.9706 & 0.0559 & 3 \\
'Alcoholic & 0.9982 & 0.6110 & 0.9970 & 0.7800 & 2 \\
pH & 0.9980 & 0.1653 & 0.9980 & 0.2129 & 2 \\
\hline
\end{tabular}

\section{LV latent variables.}

1 Squared correlation coefficient in calibration

${ }^{2}$ Root mean square error of calibration.

${ }^{3}$ Squared correlation coefficient in prediction.

4 Root mean square error of prediction.

acidity using circuit equivalent parameters were particularly remarkable. This array provides a correlating and predicting errors similar to the obtained with voltammetric e-tongue formed by modified carbon paste electrode [55] or by different families of sensitive materials [29] with which the authors have also worked.

\section{Conclusions}

The performance of an impedimetric e-tongue dedicated to the analysis of red wines has been improved using two different strategies. On one hand, sensors have been constructed using PEDOT-PSS layered nanocomposites with improved electrocatalytic properties thanks to the application of nanolayers of lutetium bisphthalocyanine $\left(\mathrm{LuPC}_{2}\right)$ and gold nanoparticles (AuNPs). On the other hand, the information extracted from the impedimetric curves has been upgraded by extracting EIS parameters from an equivalent circuit model. This improved $\mathrm{e}$ tongue is able to discriminate five red wines with very similar characteristics. In addition, using PLS-1, excellent correlations with chemical data such as Total Polyphenol Index (TPI), Folin Index, Total acidity, $\mathrm{SO}_{2}$ content, sugars, alcoholic degree and $\mathrm{pH}$ are obtained.

\section{Author contributions}

M.L. Rodriguez-Mendez and C. García Cabezon, conceived the experiments, search for funds and wrote the paper. F. Martin-Pedrosa, performed chemometrics. C. García-Hernandez and C. Salvo performed the experiments, analyzed the data with chemometrics and contributed to the manuscript writing.

\section{Conflicts of interest}

The authors declare no conflict of interest.

\section{Acknowledgments}

Financial support by MINECO and FEDER (AGL2015-67482-R) and the Junta de Castilla y León-FEDER (VA-011U16) is gratefully acknowledged. Celia Garcia-Hernandez would also like to thank the Junta de Castilla y León for a grant (BOCYL-D-24112015-9).

\section{Appendix A. Supplementary data}

Supplementary material related to this article can be found, in the online version, at doi:https://doi.org/10.1016/j.snb. 2018.09.023.

\section{References}

[1] E. O. Baldeón, M. Alcañiz, R. Masot, E.M. Fuentes, J.M. Barat, R. Grau, Voltammetry pulse array developed to determine the antioxidant activity of camu-camu (Myrciaria dubia (H.B.K.) McVaug) and tumbo (Passiffora mollisima (Kunth) L.H. Bailey) juices employing vol tammetric electronic tongues, Food Control 54 (2015)
181-187. $181-187$

[2] L. Lvova, C. Guanais Goncalves, K. Petropoulos, L. Michell, G. Volpe, D. Kirsanov, A. Legin, E. Viaggiu, R. Congestri, L Guzzella, F. Pozzoni, G. Palleschi, C. Di Natal, R. Paolesse, Electronic tongue fo:
Bioelectron. 80 (2016) 154-160.

[3] M.L. Rodriguez-Méndez, Electronic tongues for the organoleptic characterization of M.L. Rodnguez-Mendez, Electronic tongues for the organoleptlc characterization of
wines, Electronic Noses and Tongues in the Food Industry, Elsevier Academic Press, Wines, Electronic N
Amsterdam, 2016.

[4] M. Peris, L. Escuder-Gllabert, Electronic noses and tongues to assess food authenticity and adulteration, Trends Food Sci. Technol. 58 (2016) 40-54.

[5] L Escuder-Gilabert, M. Peris, Review: highlights in recent applications of electronic 5] Lescuder-Gilabert, M. Peris, Review: highlights in recent app.
tongues in food analysis, Anal. Chim. Acta 66 (2010) 15-25.

[6] C. Medina-Plaza, G. Revilla, R. Murioz, J.A. Femández-Escudero, E. Barajas, G. Medrano, J.A. De Saja, M.L. Rodriguez-Méndez, Electronic tongue formed by G. Medrano, J.A. De Saja, M.L. Rodriguez-Méndez, Electronic tongue formed by
sensors and biosensors containing phathalocyanines as electronic mediators. Application to the analysis of red grapes, J. Porphyrins Phthalacyanines 17 (2013) Applicition.

[7] M. Silwinska, C. García-Hernández, M. Koscinski, T. Dymerski, W. Wardencki. M. Sliwnska-Bartkowiak, S. Jurga, C. García-Cabezón, M.L. Rodriguez-Méndez, M. Sliwnska-Bartkowiak, S. Jurga, C. Garcia-Cabezón, M.L. Rodriguez-Mendez,
Discrimination of apple liqueurs (Nalewka) using a voltametric electronic tongue, Discrimination of apple liqueturs (Nalewka) using a voltametric electro
UV-Vis and Raman Spectroscopy, Sensors 16 (2016) 1654-1664.

[8] C. Medina-Plaza, J.A. de Saja, M.L. Rodriguez-Méndez, Bioelectronic tongue based on lipidic nanostruetured layers containing phenol oxidases and lutetium bisph-

[9] LA. Dlas, A.M. Peres, M. Vllas-Boos, M.A. Rocha, L Estevinho, A.S.C. Machado, An LA. Dias, A.M. Peres, M. Vlas-Boas, M.A. Rocha, L Estevinho, A.S.C. Machado, A ] electronic togue for honey classification, Microchim. Acta 163 (2008) 97-102. A. Riul, R.R. Malmegrim, F.J. Fonseca, L.H.C. Mattoso, An artificial taste seril
based on conducting polymers, Biosens. Bioelectron. 18 (2003) 1365-1369.

[11] A. Riul, A.M. Gallardo Soto, S.V. Mello, S. Bone, D.M.L. Taylor, H.C. Martoso, An electronic tongue using polypyrrole and polyaniline, Synth. Met. 132 (2003) 109-116.

[12] M. Ferreira, A. Riul, D. Wohnarath, F.J. Fonseca, O.N. Oliveria, L.H.C. Mattoso, $\mathrm{High}$-performance taste sensor made from Langmuir. Blodgett films of conducting polymers and a ruthenium complex, Anal. Chem. 75 (2003) 953-955.

[13] A. Riul, H.C. De Sousa, R.R. Malmegrim, D.S. Dos Santos, A.C.P.L.F. Carvalho, F.J. Fonseca, O.N. Oliverira, L.H.C. Mattoso, Wine classification by taste sensor made from ultra-thin films and using neural networks, Sens. Actuators B 98 (200-4) $77-82$.

[14] M. Cortina-Puig, X. Munoz-Berbel, M. del Valle, F.J. Munoz, M.A. Alonso-Lomillo, Characterization of an ion-selective polypyrrole coating and application to the joint determination of potassium, sodium and ammonium by electrochemical impedarice spectroscopy and partial least sçuares method, Anal. Chim. Acta 597 (2007)
231-237.

[15] M. Cortina-Puig, X.M. Munoz-Berbel, A. Alonso-Lomillo, F.J. Munoz- Pascual, M. del Valle, EIS multianalyte sensing with an automated SIA system-an electronic tongue employing the impedimetric signal, Talanta 72 (2007) 774-779,

[16] G. Ploggia, F. Dl Francesco, A. Marchetti, M. Ferro, A. Ahluwalia, A composite sensor amay impedentiometric electronic tongue part l, Charact. Biosens. Bioelectron. 22 (2007) 2618-2623.

[17] G. Pioggia, F. Di Francesco, A. Marchetti, M. Ferro, R. Leardi, A composite sensor array Impedentiometric electronic tongue Part II. Discrimination of basic tastes, Biosens. Bloelectron. 22 (2007) 2624-2628

[18] R. Masot, M. Alcaniz, A. Fuentes, F.C. Schmidt, J.M. Barat, L. Gil, D. Baigts, R. Martinez-Manez, J. Soto, Design of a low-cost nondestructive system for punc. tual measurements of salt levels in food products using impedance spectroscopy. Sens. Actuators A 158 (2010) 217-223.

[19] J.A. Serra, F.C. Schmidt, A. Fuentes, J.M. Barat, R. Grau, R. Masot, M. Alcafiz, Desarrollo de un método de medida rápido para el control del contenido en humedad y sal en queso, VI Congreso Iberoamericano de Ingeniería de Alimentos CIBIAVII (2009)

[20] H.J. Swatland, F. Darkin, S.J. Naylor, L. Caston, R.D. Moccia, Muscle colour de velopment in Artic charr, Salvellnus alpinus (L), monitored by fbre-optics and electrical impedance, Aquacult. Res. 29 (1998) 367-372. 
Paper VII: Impedimetric electronic tongue based on nanocomposites for the analysis of red wines. Improving the variable selection method.

[21] P.A. Ulloa, R. Guerra, A.M. Cavaco, A.M.R. da Costa, A.C. Figueria, A.F. Brigas, Determination of the botanical origin of honey by sensor fusion of Impedance e Determination of the botanical orligin of honey by sensor fusion of Impedance

[22] J. Yanez Herasa, D. Pallarola, F. Bartaglinl, Electronic tongue for simultaneous detection of endotoxins and other contaminants of microbiological origin, Biosens. Bioelectron. 25 (2010) 2470-2476.

[23] F.P.A. Cabral, B.B. Bergamo, C.A.R. Dantas, A. Riul, F.A. Glacomettl, Impedance e tongue instrument for rapid linuid assessment, Rev. Sei. Instrum. $80(2009) 026107$ $1-026107-3$.

[24] C.A. Olivati, A. Riul, D.T. Balogh, ON. Oliveim, M. Ferreim, Detection of phenolic compounds using Impedance spectroscopy measurements, Bloprocess Blosyst. Eng. 32 (2009) 41-46.

[25] A.P. Bhondekar, M. Dhiman, A. Sharma, A. Bhakta, A. Ganguli, S.S. Bari, R. Vig,

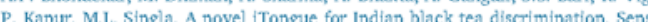
Actuators B $148(2010) 601-609$

[26] A. Riul, H.C. de Sousa, R.R. Malmegrim, D.S. dos Santos, A.C.P.L.F. Carvalho, F.J. Fonseca, O.N. Oliveira, L.H.C. Mattoso, Wine classification by taste sensors made fiom ultra thin films and using neural networks, Sens Actuators B 98 (2004) $77-82$.

[27] P. Giménez-Gómez, R. Fscudé-Pujol, F. Capdebila, A. Puig-Pujol, C. Jimenez. Jorquera, M. Gutiérrez-Capitan, Portable electronic tongue based on microsensors for the analysis of cava wines, Sensors 16 (2016) 1796-1802.

[28] G. Verrelli, L. Lvova, R. Paolesse, C. Di Natale, A. D'Amico, Metalloporphyria based electronic tongue an application for the analysis of italian white wines, Seneors 7

[29] V. Parra, A. Arrieta, J.A. Fernández-Escudero, M. Iñiguę, J.A. De Saja, M.L Rodriguez-Méndez, Monitoring of the ageing of red wines in oalk barrels by means of an hybrid electronic tongue, Anal. Chim. Acta 563 (2006) 229-237.

[30] J. Wu, J. Liu, M. Fu, J. Li, Z. Lou, Classification of chinese yellow wines by che mometric analysis of cyclic voltaummogram of copper electrodes, Sensors 12 (2005) $529-536$.

[31] C. Medina-Plaza, C. García-Hernandez, J.A. De Saja, J.A. Fernández-Escudero, F. Barajas, G. Medrano, C. García Cabezón, F. Martín-Pedrosa, M.I. RodriguezMendez. The advantages of disposable screen-printed biosensors in a bioelectronic tongue for the analysis of grapes, LWT Food Scl. Technol. 62 (2015) 940-947.

[32] I.M. Apetrei, M.I. Rodríguez.Méndez, C. Apetrei, I. Nevares, M. del Alamo, J.A. de Saja, Monitoring of evolution during red wine aging in oak barrels and altemative method by means of an electronic panel test, Food Res. Int. 45 (2012) 244-249.

[33] L.N. Furinh, E Feitosa, P. Alessio, M.H Shimabuikuro, A Riul, C.J. Constantino, Tuning the nanostructu/nickel tetrasulfonated phthalocyanine bilayer in LBL films, Mater. Sci. Eng. C 32 (2013) 2937-2946.

[34] C. Garcia-Hernandez, C. Garcia-Cabezon, F. Martin-Pedrosa, J.A. De Saja, M.L. Rodriguez-Mendez, Layered composites of PEDOT/PSS/nanoparticles and PEDOT/PSS/phthalocyanines as electron mediators for sensors and biosensors, Beilstein J. Nanotechnol. 7 (2016) 1948-1959.

[35] M.H.M. Facure, L.A. Mercante, L.H.C. Mattoso, D.S. Correa, Detection of trace levels of organophosphate pesticides using an electronic tongue based on graphene hybrid narocomposites, Talanta 167 (2017) 59-66.

[36] J. Zhang, J. Xu, W. Wen, Z. Wang, H. Zhang, W. Ding, Voltametric detennination of phytoinhibitor maleic hydrazine using PEDOT-PSS composite electrode, J. Electroanal. Chem. 751 (2015) 65-74.

[37] V. Sethuraman, P.J. Muthuraja, A. Raj, P. Manisankar, A highly sensitive electrochemical blosensor for catechol using conducting reduced graphene oxide-metal oxide enzyme modified electrode, Biosens. Bioelectron. 84 (2016) 112-119,

[38] S.W. Dutse, N.A. Yusof, H. Ahmad, Improving the sensitivity of Pedot:Pss modified gold electrode using gold and silver nanoparticles for Ganoderma boninense DNA detection, Sens. Transduct. 191 (2015) 46-51.

[39] R.M. Reis, R.B. Valim, R.S. Rocha, A.S. Lima, P.S. Castro, M. Bertotti, M.R.V. Lanza, The use of copper and cobalt phthalocyanines as electrocatalysts for the oxyger reduction reaction in acid medium, Electrochim. Acta 139 (2014) 1-6.

[40] P.A. Antunes, C.M. Santana, R.F. Aroca, O.N. Oliveira, C.J.L. Constantino, A. Riul, The use of Langmulr-Blodgett fllms of a perylene derlvative and polypyrrole in the detection of trace levels of Cu2 + ions, Synth. Met. 148 (2005) 21-24.

[41] C. Garcia-Hemandez, C. Garcia-Cabezon, C. Medina-Plaza, F. Martin-Pedrosa, Y. Blanco, J.A. de Saja, M.I. Rodriguez-Mendez, Electrochemical behavior of
polypyrrol/AuNP composites deposited by different electrochemical methods

sensing properties towards catechol, Bellsteln J. Nanotechnol. 6 (2015) 2052-2061. [42] J.W. Slot, H.J. Geuze, A new method of preparing gold probes for multiple-labelling

A. Gonglen Jib del Valle, G Alcaide, M.I. RodriguezMendez, Improvement of the synthesis of lutetium bisphthalocyanine using the Taguchl method, Analyst $125(2000)$ 341-346.

[44] OIV Organisation Internationale de la Vigne et du Vin, Compendium of International Methods of Analysis of Wines and Musts, Paris, (2013)

[45] J. Nevrela, M. Micjan, M. Novota, S. Kovacova, M. Pavuk, P. Juhasz, J. Kocac J. Jakaboric, M. Wels, Secondary doping in poly(3,4 ethylenedloxythlophene):poly (4 styremesulfonate) thin flms, Polym. Phys 53 (2015) $1139-1146$.

[46] M. Bouvet, P. Gaudillat, J.M.J. Suisse, Phthal ocyanine-based hybrid materials for chemosensing, J. Porphyrins Phthalocyanines 17 (2013) 913-919.

[47] A. Guerrero, M. Haro, S. Bellani, M.R. Antognazza, L. Meda, S. Gimenez, J. Bisquer, Organic photoelectrochemical cells with quantitative photocarrier conversion, Energy Enviros Sci. 7 (2014) 3666-3673.

[48] V.F. Lvovich, Impedance Spectroscopy: Applications to Electrochemical and Dielectric Phenomena, John Wiley \& Sons, New Jersey, 2012.

[49] A.L. Rinali, R. Carballo, Impedimetric non-enzymatic glucose sensor based on nickel hydroxide thin film onto gold electrode, Sens. Actuators B 228 (2016) 43-52.

[50] A. Maikap, K. Mukherjee, B. Mondal, Zinc oxide thin film based nonenzymatic electrochemical sensor for the detection of trace level catechol, RSC Adv. 69 (2016) $64611-64616$

[51] Z.O. Uygun, Y. Dilgin, A novel impedimetric sensor based on molecularly imprinted polypyrrole modified pencil graphite electrode for trace level determination of polypyrrole modified pencil graphite electrode for

[52] Xu H. Gorgy, K. Gondran, A. Le Goff, N. Spinelli, C. Lopez, Label-fiee impedimetric thrombin sensor based on poly(pyrrole-nitrilotriacetic acid)-aptamer film, Biosensors and Bioelectronic 41 (2013) 90-95.

[53] C. Chandra, H. Lang, D. Bahadur, Polyaniline-iron oxide nanohybrid film as multifunctional label-fiee electrochemical and blomagnetic sensor for catechol, Anal. Chim. Acta 795 (2013) 8-16.

[54] S. Mondal, M.V. Sangaranarayananet, A novel non-enzymatic sensor for urea using a polypyrrole-coated platinum electrode, Sens. Actuators B 177 (2013) 478-486.

[55] V. Parra, A. Arrieta, J.A. Fernández.Escudero, M.L. Rodríguez.Méndez, J.A. De Saja, Electronic tongue based on chemically modifed electrodes and voltammerty for the detection of adulterations in wines, Sens. Actuators B 118 (2006) 448-453.

Celia Garcia-Hernández obtalned the Ms in Nanoscience in 2015 (U. of Valladolid. Spain). She is currently working on her PhD Thesis which is dedicated to the development of electrochemical sensors for the analysis of antioxidants. She is author of 9 scientific papers.

Coral Salvo-Cominoz obtained the Ms in Analytical Chemistry in 2015 (U. Complutense Madrid. Spain). She is currently working on her PhD Thesis which is dedicated to the development of electrochemical sensors for the analysis of antioxidants. She is author of scientific papers.

Fernando Martin-Pedrosa is full professor at the University of Valladolid and Head of ts dies of different solld materlals. He is author of more than 80 papers.

Maria Luz Rodriguez-Mendez is Full professor of horganic Chemistry at the Engineer school of the University of Valladolid and Head of the group of sensors UVASens. Currently she is involved in several funded Projects devoted to the development of arrays of voltammetric nanostructured sensors and biosensors for the characterization of foods and to the assessment of their antioxidant potential. She is author or co-author of over 145 publications, seven books and three patents in the field

Cristina Garcia Cabezon, is assistant professor at the Engineers school of the University of Valladolid. She is an expert in electrochemistry and impedance spectroscopy. She is author or coauthor of more than 40 papers in the field 


\subsection{Paper VIII}

An electrochemical quartz crystal microbalance multisensor system based on phthalocyanine nanostructured films: Discrimination of musts.

By Celia García-Hernández, Cristina Medina-Plaza, Cristina GarcíaCabezón, Fernando Martín-Pedrosa, Isabel del Valle, José Antonio de Saja and María Luz Rodríguez-Méndez.

Sensors 15 (2015) 29233-29249.

(doi: 10.3390/s151129233) 


\subsubsection{Motivation and objective}

Quartz crystal microbalance (QCM) is a simple, high-resolution gravimetric technique with no specific selectivity. Combining electrochemistry and QCM is denoted as electrochemical quartz crystal microbalance (EQCM). In EQCM assays one can simultaneously register the electrochemical response and mass changes that accompany the oxidation/reduction of electroactive species. Therefore, electrochemical quartz crystal microbalance (EQCM) sensors can be an alternative or complement to the traditional transducers implemented in electronic tongues (mainly potentiometric, voltammetric and amprerometric).

Most applications for QCM sensors are in electronic noses for the analysis of volatile organic compounds (VOCs). The sensor group of Prof. Di Natale and Prof. Paolesse is one of the most active in the field of QCM and they have designed a commercial electronic nose based on QCM sensors coated with porphyrins for the analysis of organic gases and vapors. QCM sensors have also been applied in several applications, such as piezoelectric immunosensors, for the recognition of biomolecular interactions. In addition, EQCM has been used as a gravimetric sensor in many types of electrochemical studies, including: metal deposition; corrosion studies; oxide formation; dissolution studies (e.g., phenol oxidation); surfactant adsorption/desorption; enzyme adsorption/desorption; changes in the conductivity of polymer films during redox processes; electropolymerization studies; ion insertion studies; or load transfer studies during redox processes (e.g., phthalocyanines). However, there are no works reporting the use of an EQCM multisensor system implemented as an electronic tongue in the analysis of food.

When no sensing element is added, QCM works just like a transducer. Therefore, in several works, QCM electrode surfaces have been modified with various chemicals and biomaterials to obtain selectivity and specificity. A careful choice of the sensing material is essential for achieving high performance sensor arrays, such as those used in nanostructured electronic tongues. As already mentioned, phtaholcyanines are good candidates due to their excellent electrocatalytic properties. Moreover, the use of nanostructured films has played an important role in increasing sensitivity as of the number of active sites and the intimate contact with the analyte are greatly improved by the use of thin films.

Herein, we have developed an electronic tongue based on EQCM sensors, modified with metallophthalocyanines, using the LbL technique. The multisensor system as-prepared was able 
to discriminate musts with different phenolic and sugar contents. The response of the array of sensors for must samples was studied by simultaneously recording cyclic voltammetry and mass changes. The capability of the system to discriminate between grapes of different varieties was evaluated by means of parallel factor analysis (PARAFAC). The relationship between the voltammetric or mass signals with the results obtained by chemical analysis was studied by means of multi-way partial least squares (N-PLS).

\subsubsection{Summary of the research work}

Sensors were prepared by depositing 20 thin film bilayers onto a quartz crystal coated with platinum by means of the LbL technique. For this purpose, we used metalphthalocyanines as the anionic layers and polyallylamine hydrochloride (PAH) as the polycation. Iron(III) phthalocyanine tetrasulfonic acid monosodium salt (FePcSO3-), copper(II) phthalocyanine tetrasulfonic acid tetrasodium salt (CuPc ${ }^{\mathrm{SO}}{ }^{-}$) and nickel(II) phthalocyanine tetrasulfonic acid tetrasodium salt ( $\mathrm{NiPc}^{\mathrm{SO}}{ }^{-}$) were used to achieve an array of partially selective sensors with overlapping specificities. The quality of the LbL films was monitored by UV-Vis following the characteristic Q band of the metallophthalocyanie at 620-640 nm, which confirmed a linear relationship between the number of layers and the absorbance of the $\mathrm{Q}$ band and, therefore, $\mathrm{a}$ uniform growth of the LbL films and good quality in the deposition.

The red grape samples analyzed, harvested in September 2013, were provided by the the vineyards Bodega Cooperativa de Cigales and the Instituto Tecnológico Agrario de Castilla y León (ITACYL). The chemical parameters of the corresponding red grapes, analyzed according to international regulations, including sugar content, ${ }^{\circ}$ Brix, total polyphenolic content (TPI) and degree 16.8, were provided by the Oenological Centre of Castilla y León. In addition, the FolinCiocalteu method was carried out to evaluate the phenolic content by means of another methodology different from TPI.

The electrochemical experiments were carried out with a quartz crystal microbalance and working electrodes were quartz crystals coated with platinum (5 MHz, AT-cut planoconvex) with the $L b L$ films onto the surface as sensitive and electrocatalytic material. Before performing the experiments, the quartz crystal microbalance was calibrated according to the corresponding procedure. For this purpose, a galvanostatic deposition of copper onto the surface of the crystals 
was applied in order to determine the Sauerbrey constant of the quartz crystals coated with platinum. Then, cyclic voltammetry experiments were carried out and mass variations were simultaneously registered. In order to know the electrochemical responses and register mass variations for phenols and sugars, the EQCM sensors were tested in catechol, which is an antioxidant usually found in grapes and wines, and glucose, one of the major sugars.

Voltammograms registered in catechol showed two redox processes corresponding to catechol and the decomposition of water. Depending on the metallophthalocyanine used, the peak position and intensities were different for each one, although the shapes of the voltammograms were similar. The intensities of the as-prepared EQCM sensors were higher than those observed in the bare platinum quartz crystal, confirming the electrocatalytic effect of the phthalocyanines. It was observed that the intensities of the oxidation peaks were higher when the concentration of catechol increased; whereas the intensities in the reduction peaks decreased. The position of the peaks demonstrated that the electrocatalytic effect of the sensors decreased when advancing in the transition metal series ( $\left.\mathrm{FePc}^{\mathrm{SO}}<\mathrm{NiPc} \mathrm{NO}<\mathrm{CuPc}^{\mathrm{SO} 3}\right)$. Mass variation signals showed that mass increased during the oxidation of catechol and decreased during the reduction. Voltammograms registered in glucose were poor and did not show any redox peak, except for the peaks associated to protons at negative potentials, whereas the massograms evidenced a mass increase that was attributed to the adhesion of the sugar onto the surface. The mass deposited onto the EQCM sensors increased when the concentration of glucose increased.

Due to the grapes having different phenolic and sugar compositions, depending on the variety of grape, the as-prepared EQCM sensors were good candidates to analyze different grape musts, so they were immersed in six different Spanish grape musts (Juan García, Prieto Picudo, Mencía Regadío, Cabernet Sauvignon, Garnacha and Tempranillo). The voltammograms and massograms registered in must samples were different from one grape to another, but in general, the voltammograms showed a broad anodic peak that could be associated to the polyphenolic content in grapes and wines and the massograms showed a progressive increase in mass which was associated with the adhesion of sugars onto the crystal surface. The richness and crossselectivity of the responses were successfully used to discriminate between the grape samples by means of PARAFAC (parallel factor analysis), a non-supervised multivariate technique. The PARAFAC score plot obtained from the voltammogram analysis showed that samples can be discriminated according to the Total Polyphenolic Index (TPI). When analyzing the mass outputs 
for grape musts, the PARAFAC score plot showed that samples were located according to the Brix degree (a sugar content parameter).

With the purpose of establishing correlations between the chemical parameters provided by the Oenological Centre of Castilla y León and the data obtained with the EQCM array in must samples, N-PLS (multi-way partial least squares) were carried out. The prediction models obtained confirmed that the voltammetric data presented a better correlation with the parameters related to the phenolic content (TPI and Folin-Ciocalteau index), whereas the mass data had good correlations with the sugar parameters (sugar content and ${ }^{\circ}$ Brix), so the results obtained with the PARAFAC analysis were confirmed. 



\title{
An Electrochemical Quartz Crystal Microbalance Multisensor System Based on Phthalocyanine Nanostructured Films: Discrimination of Musts
}

\author{
Celia Garcia-Hernandez ${ }^{1}$, Cristina Medina-Plaza ${ }^{1}$, Cristina Garcia-Cabezon ${ }^{2}$, \\ Fernando Martin-Pedrosa ${ }^{2}$, Isabel del Valle ${ }^{3}$, Jose Antonio de Saja ${ }^{4}$ and \\ Maria Luz Rodríguez-Méndez ${ }^{1, *}$
}

1 Department of Inorganic Chemistry, Engineers School, University of Valladolid, Valladolid 47011, Spain; E-Mails: Celiagarciahernandez@gmail.com (C.G.-H.); Crismedina@eii.uva.es (C.M.-P.)

2 Department of Materials Science, Engineers School, University of Valladolid, Valladolid 47011, Spain; E-Mails: crigar@eii.uva.es (C.G.-C.); fmp@eii.uva.es (F.M.-P.)

3 Department of Electronic Technology, Engineers School, University of Valladolid, Valladolid 47011, Spain; E-Mail: isaval@eii.uva.es

4 Department of Condensed Matter Physics, Faculty of Sciences, University of Valladolid, Valladolid 47011, Spain; E-Mail: sajasaez@fmc.uva.es

* Author to whom correspondence should be addressed; E-Mail: mluz@eii.uva.es; Tel.: +34-983-423-540; Fax: +34-983-423-310.

Academic Editor: W. Rudolf Seitz

Received: 10 September 2015 / Accepted: 13 November 2015 / Published: 19 November 2015

\begin{abstract}
An array of electrochemical quartz crystal electrodes (EQCM) modified with nanostructured films based on phthalocyanines was developed and used to discriminate musts prepared from different varieties of grapes. Nanostructured films of iron, nickel and copper phthalocyanines were deposited on Pt/quartz crystals through the Layer by Layer technique by alternating layers of the corresponding phthalocyanine and poly-allylamine hydrochloride. Simultaneous electrochemical and mass measurements were used to study the mass changes accompanying the oxidation of electroactive species present in must samples obtained from six Spanish varieties of grapes (Juan García, Prieto Picudo, Mencía Regadio, Cabernet Sauvignon, Garnacha and Tempranillo). The mass and voltammetric outputs were processed using three-way models. Parallel Factor Analysis (PARAFAC) was successfully used to discriminate the must samples according to their
\end{abstract}


variety. Multi-way partial least squares (N-PLS) evidenced the correlations existing between the voltammetric data and the polyphenolic content measured by chemical methods. Similarly, N-PLS showed a correlation between mass outputs and parameters related to the sugar content. These results demonstrated that electronic tongues based on arrays of EQCM sensors can offer advantages over arrays of mass or voltammetric sensors used separately.

Keywords: LbL; EQCM; phthalocyanine; must; grapes; electronic tongue

\section{Introduction}

The combination of an array of partially selective sensors with overlapping specificities with advanced mathematical signal-processing methods has yielded a new sensing technology for chemical analysis in liquid media, the so called electronic tongue (ET) [1,2]. ETs are holistic systems that provide global and qualitative information about samples instead of quantitative data about specific compounds. However, if the data matrix obtained by such multisensor systems is analyzed with adequate chemometric processing tools, descriptive or predictive information of particular parameters can be extracted $[3,4]$.

Arrays of sensors dedicated to the analysis of liquids are usually based on electrochemical sensors including potentiometric [5], amperometric [6], voltammetric [7-9] or impedimetric ones [10]. Many efforts have been dedicated to the discrimination of wines and musts using electronic tongues $[2,3,8,9,11-14]$. Voltammetric electrodes chemically modified with electrocatalytic materials are particularly suitable for the analysis of wines because voltammograms contain information related to the redox and ionic species present in wines [15]. For this reason, e-tongues based on voltammetry have been able to discriminate red wines with different antioxidant capability [16], wines elaborated with different varieties of grapes [17], to detect adulterations [8] or to follow the ageing of red wines using different methods [18].

The intrinsic complexity, richness and cross-selectivity of the signals generated by voltammetric sensor arrays are an advantage because the datasets contain meaningful information about the samples. Using partial least squares regression analysis (PLS), correlations could be established between the voltammetric curves (not only specific peaks) and the polyphenol content, or the acidity of red wines $[12,16,19]$. Finally, multi-transduction systems are more and more popular, since they combine different classes of sensors that provide complementary information [13,20-24].

Quartz Crystal Microbalances (QCM) are a different approach where the sensing element is a coated resonator. The interaction with the sample results in a change in the mass of the crystal which affects the frequency at which the crystal oscillates. QCM sensors modified with a variety of materials (porphyrins, CNTs, calixarenes and biomolecules, among others) have been widely used in electronic noses to analyze vapors with sub-nanogram sensitivity [25-28]. QCMs can also work in an aqueous environment and they have been able to detect a variety of substances in solution $[29,30]$. However, only few examples of electronic tongues based on QCM sensors for the analysis of liquids have been reported [31-33]. 
There are numerous methods to functionalize QCM surfaces. They include hydrophobic bonds, ionic bonds, hydrogen bonds or electrostatic interactions, among others [34]. Nanostructured films show enhanced surface to volume ratios that can increase the sensitivity of the sensors [35]. In fact, QCM sensors have been prepared using Langmuir-Blodgett (LB) [36], Layer by Layer (LbL) [37], Self-Assembling Monolayer (SAM) [38], Electrodeposition (EDP) [39,40], Plasma-Polymerized Films (PPF) [41] or Molecular Imprint (MIP) [42] techniques.

Apart from their sub-nanogram sensitivity, another possible advantage of QCM sensors for the analysis of liquids, is that the same device can be used to obtain simultaneously mass changes and electrochemical signals. For this reason, the Electrochemical Quartz Crystal Microbalance (EQCM) has become a well-established technique for the investigation of mass changes associated with electrochemical surface processes such as adsorption of enzymes [43], electrodeposition [44], electropolymerization [45], ion insertion [46] or redox processes [33]. In this way, EQCM can provide higher amounts of information about a sample than electrochemistry or mass measurements performed separately $[33,47,48]$.

EQCM sensors covered with phthalocyanines have been successfully used to analyze phenols present in wines [33]. The reason for their success is that phthalocyanines show electrocatalytic properties towards a variety of analytes including phenols [33,49,50]. In addition, nanostructured electrodes based on phthalocyanines can be prepared by electrodeposition [40], the Langmuir-Blodgett (LB) $[35,51]$ or the Layer by Layer (LbL) technique [52-54] on different substrates such as indium tin oxide (ITO) or platinum.

The objective of this work was to combine EQCM sensors to form an array able to discriminate musts with different phenolic content, obtained from different varieties of Spanish grapes. For this purpose, QCM substrates were modified with three different metallophthalocyanines using the LbL technique. The response of the array of sensors towards the must samples was studied by recording cyclic voltammetry and mass changes synchronously. The capability of the system to discriminate grapes of different varieties was evaluated by means of parallel factor analysis (PARAFAC). The relationship between the voltammetric or the mass signals with the results obtained by chemical analysis was studied by means of multi-way partial least squares (N-PLS). The complementarity of the information provided by the electrochemical and mass outputs was discussed.

\section{Experimental Section}

\subsection{Chemicals and Grape Samples}

All chemicals and solvents were purchased from Sigma-Aldrich (St. Louis, MO, USA) and used without further purification. The solutions were obtained by dissolving substances in deionized water (resistivity of $18.2 \mathrm{M} \Omega \cdot \mathrm{cm}^{-1}$ ) acquired from a Milli-Q system (Millipore, Billerica, MA, USA).

Three water soluble tetrasulfonate acid metallophthalocyanines ( $\mathrm{MPc}^{\mathrm{SO}}$ ) were used as the anions in the LbL films. They included iron(III) phthalocyanine tetrasulfonic acid monosodium salt, copper(II) phthalocyanine tetrasulfonic acid tetrasodium salt, and nickel(II) phthalocyanine tetrasulfonic acid tetrasodium salt; (denoted as $\mathrm{FePc}^{\mathrm{SO}}, \mathrm{NiPc}^{\mathrm{SO} 3}$ and $\mathrm{CuPc}^{\mathrm{SO} 3}$ respectively). Polyallylamine hydrochloride $(\mathrm{PAH})$, was used as the polycation in LbL films. 
Six varieties of red grapes were included in the study (Juan García, Tempranillo, Prieto Picudo, Mencía Regadío, Cabernet, Garnacha). They were harvested in September 2013 from the vineyards "Bodega Cooperativa de Cigales" and "Instituto Tecnológico Agrario de Castilla y León (ITACYL)", both located in the Valladolid area of Castilla y León in Spain. To obtain the musts, 200 berries were introduced in a plastic bag and crushed for one minute. The Oenological Centre of Castilla y León carried out the chemical analysis following international regulations [55]. Parameters analyzed included the typical indicators of the glucose content: sugar content $(\mathrm{g} / \mathrm{L})$, degree 16.8 and Brix degree. The classical indicator of the polyphenolic content, the Total Polyphenol Index (TPI) was also analyzed. The polyphenolic content was also evaluated using the Folin-Ciocalteau method. The results are collected in Table 1.

Table 1. Results of the chemical analysis carried out by traditional chemical methods.

\begin{tabular}{cccccc}
\hline Grape Variety & $\begin{array}{c}\text { Sugar Content } \\
\text { (g/L) }\end{array}$ & Brix Degree & $\begin{array}{c}\text { Total Polyphenol } \\
\text { Index. TPI }\end{array}$ & Degree 16.8 & $\begin{array}{c}\text { Polyphenol Content. Folin-Ciocalteau Method } \\
\text { (g/L) }\end{array}$ \\
\hline Prieto Picudo & 224.1 & 22.89 & 19 & 13.31 & 0.46 \\
Garnacha & 187.4 & 19.68 & 15 & 11.13 & 0.38 \\
Cabcrnet-Sauvignon & 246.4 & 24.75 & 28 & 14.64 & 0.62 \\
Tempranillo & 209.1 & 21.53 & 28 & 12.42 & 0.52 \\
Juan García & 216.0 & 22.18 & 29 & 12.83 & 0.69 \\
Mencía Regadio & 203.3 & 21.05 & 23 & 12.08 & 0.54 \\
\hline
\end{tabular}

\subsection{Electrochemical Measurements}

Electrochemical quartz crystal microbalance experiments were carried out with a quartz crystal microbalance (QCM200 + QCM25 Crystal Oscillator, purchased from Standford Research Systems, Sunnyvale, CA, USA) connected to a Parstat 2273 potentiostat/galvanostat (EG\&G, Oak Ridge, TN, USA). The mass-sensitive oscillators were $2.54 \mathrm{~cm}$ diameter, $5 \mathrm{MHz}$ AT-cut planoconvex quartz crystals coated with platinum.

The oscillators were used simultaneously to register mass changes and as the working electrode of the electrochemical measurements. PC software displays the relative frequency changes in synchronicity with the electrochemical data.

Cyclic voltammetry was carried out using a conventional three-electrode cell. The reference electrode was $\mathrm{Ag} \mid \mathrm{AgCl} / \mathrm{KCl} 3 \mathrm{~mol} \cdot \mathrm{L}^{-1}$ and the counter electrode was a platinum sheet with a surface of $1 \mathrm{~cm}^{2}$. Cyclic voltammograms were registered at a sweep rate of $0.1 \mathrm{~V} \cdot \mathrm{s}^{-1}$. The variations of mass taking place simultaneously to the cyclic voltammetry experiment were registered. All the samples were measured seven times with each sensor to check the robustness of the experiments.

\subsection{Calibration of the Quartz Crystal}

A calibration of the quartz crystal was carried out to determine the sensitivity factor (also called Sauerbrey constant) by galvanostatic deposition of copper on the platinum surface. Sauerbrey constants have been used to calculate mass shifts during the experiment (Equation (1)):

$$
\Delta f=-C_{f} \cdot \Delta m
$$


where $\Delta f$ is the observed frequency change $(\mathrm{Hz}), C_{f}$ is the Sauerbrey constant $\left(56.6 \mathrm{~Hz} \cdot \mathrm{cm}^{2} \cdot \mu \mathrm{g}^{-1}\right.$ for a $5 \mathrm{MHz}$ At-cut quartz crystal at room temperature) and $\Delta m$ is the change in mass per unit area $\left(\mu \mathrm{g} \cdot \mathrm{cm}^{-2}\right)$.

A $0.5 \mathrm{~mol} \cdot \mathrm{L}^{-1}$ solution of $\mathrm{CuSO}_{4}$ in $0.1 \mathrm{~mol} \cdot \mathrm{L}^{-1} \mathrm{H}_{2} \mathrm{SO}_{4}(\mathrm{pH}=1.37)$ was used to deposit copper on the platinum electrode using chronopotentiometry $(-2 \mathrm{~mA}, 70 \mathrm{~s})$. The plot of the frequency shift $v s$. charge showed excellent linearity $\left(y=7 \times 10^{-5} x-0.0632 ; R^{2}=0.9996\right)$. According to the linear plot obtained in the calibration process, the Sauerbrey constant of the quartz crystal obtained was $59.4 \mathrm{~Hz} \cdot \mathrm{cm}^{2} \cdot \mu \mathrm{g}^{-1}$, which is consistent with the theoretical value of $56.6 \mathrm{~Hz} \cdot \mathrm{cm}^{2} \cdot \mu^{-1}$.

\subsection{Sensor Preparation}

The platinum-coated quartz crystal substrates were cleaned using a mixture of $\mathrm{H}_{2} \mathrm{SO}_{4}$ and $\mathrm{H}_{2} \mathrm{O}_{2}$ (piranha solution; 3:1 mixture of sulfuric acid and 30\% hydrogen peroxide) and thoroughly rinsed in deionized water (resistivity of $18.2 \mathrm{M} \Omega \cdot \mathrm{cm}^{-1}$ ) before use.

The LbL films were grown using PAH as the positive layer and iron, nickel or copper tetrasulfonate phthalocyanines $\left(\mathrm{FePc}^{\mathrm{SO} 3}, \mathrm{NiPc}^{\mathrm{SO} 3}\right.$ or $\mathrm{CuPc}^{\mathrm{SO} 3}$ ) as the negative layer using a previously published procedure [56]. Water solutions of PAH $\left(0.5 \mathrm{~g} \cdot \mathrm{L}^{-1}\right)$ and of the corresponding phthalocyanine $\left(0.05 \mathrm{~g} \cdot \mathrm{L}^{-1}\right)$ were used to build the films. LbL films were fabricated by successive immersions of the QCM substrate in the PAH and phthalocyanine solutions. The following sequence of immersions was followed: (1) PAH solution (5 min); (2) deionized water gently stirred to remove excess of non-adsorbed $\mathrm{PAH}$ ( $1 \mathrm{~min}$ ); (3) $\mathrm{MPc}^{\mathrm{SO} 3}$ solution (5 min); (4) deionized water gently stirred to remove excess and non-adsorbed $\mathrm{MPc}^{\mathrm{SO} 3}(1 \mathrm{~min})$. After these four steps a bilayer was formed and more layers are grown by repeating the sequence. After each electrochemical measurement, QCM substrates were cleaned by immersing the $\mathrm{Pt} /$ quartz device in $\mathrm{HNO}_{3} 60 \%$ (sonication during $1 \mathrm{~min}$ ) to remove $\mathrm{LbL}$ films. QCM devices were then rinsed in deionized water and dried with nitrogen gas.

\subsection{UV-Visible Characterization}

The growth of LbL films was monitored with UV-Vis absorption spectroscopy (UV-2600 model, purchased from Shimadzu, Kyoto, Japan) by testing the linearity between the number of layers deposited and the absorbance.

\subsection{Multisensor System Statistical Analysis}

A multisensor system was constructed using the signals obtained from four sensors: the already described $\mathrm{FePc}^{\mathrm{SO}} / \mathrm{PAH}, \mathrm{NiPc}^{\mathrm{SO} 3} / \mathrm{PAH}$ and $\mathrm{CuPc}^{\mathrm{SO}} / \mathrm{PAH} \mathrm{LbL}$ films and a bare Pt quartz crystal microbalance. The multivariate data analysis was performed by using Matlab v2014b (The Mathworks Inc., Natick, MA, USA) and The Unscrambler (CAMO Software AS, Oslo, Norway). Voltammograms and massograms were pre-processed by using an adaptation of a data reduction technique based on predefined response "bell-shaped-windowing" curves called "kernels" [15,57]. Using this method, 10 variables were obtained from each voltammogram and from each massogram. These data were used as the input variable in statistical analysis. A non-supervised multivariate method, the Parallel Factor Analysis (PARAFAC) was used to analyze the curves and to evaluate the capability of discrimination 
of the array of sensors. Multi-way partial least squares (N-PLS) was used to evidence the correlations between the voltammetric and mass outputs and the chemical indicators.

\section{Results and Discussion}

\subsection{UV-Visible characterization}

$\mathrm{LbL}$ films were prepared using the four-step sequence described above. The procedure was repeated 20 times to obtain 20 bilayer-thick films. The growth of bilayers was monitored every four bilayers by UV-vis absorption spectroscopy. The characteristic B band at shorter wavelengths and Q band at longer wavelengths, which are attributed to $\mathrm{HOMO} \rightarrow \mathrm{LUMO}$ electronic transitions of the $\pi$ electrons of the Pc ring, could be clearly seen. The features observed in the UV-Vis absorption spectra are assigned to the $\mathrm{MPc}^{\mathrm{SO} 3}$ since $\mathrm{PAH}$ does not absorb within this wavelength range.

The absorbance of the $\mathrm{Q}$ band increased linearly with the number of bilayers. This is illustrated in Figure 1 for the $\mathrm{CuPc}^{\mathrm{SO}} / \mathrm{PAH}$ film where the absorbance at $620 \mathrm{~nm}$ vs. the number of deposited bilayers is represented, confirming the good quality of the deposition. This shows that a similar amount of material is transferred onto the substrate per deposited layer confirming a uniform growth of the LbL films. The quality of the layers (and hence the linearity regression coefficient) decreased when more than 20 bilayers were deposited. For this reason, further studies were carried out with 20 bilayers.

Similar results were obtained with the three phthalocyanines tested and the only difference was the value of the $\mathrm{Q}$ band position $\left(640 \mathrm{~nm}\right.$ for $\mathrm{FePc}^{\mathrm{SO} 3} / \mathrm{PAH}, 620 \mathrm{~nm}$ for $\mathrm{NiPc}^{\mathrm{SO} 3} / \mathrm{PAH}$ and 620 for $\left.\mathrm{CuPc}^{\mathrm{SO}} / \mathrm{PAH}\right)$, which are in good agreement with previous results $[53,54]$.

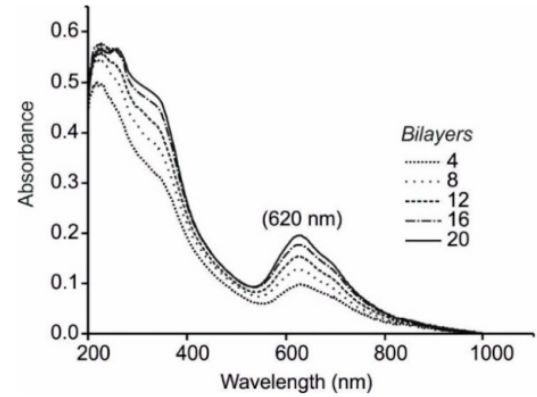

(a)

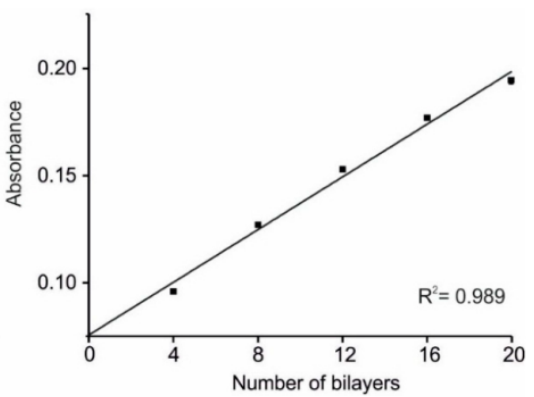

(b)

Figure 1. UV-Vis characterization of $4-20 \mathrm{CuPc}^{\mathrm{SO}} / \mathrm{PAH} \mathrm{LbL}$ bilayers. (a) UV-Vis absorption spectra; (b) Linear correlation between absorbance $v s$. number of bilayers.

In addition, the absorbance values registered increased when advancing in the transition metal series ( $\mathrm{FePc}^{\mathrm{SO}} / \mathrm{PAH}<\mathrm{NiPc}^{\mathrm{SO}} / \mathrm{PAH}<\mathrm{CuPc}^{\mathrm{SO} 3} / \mathrm{PAH}$ films) (for instance, the values of $\mathrm{Q}$ band absorbance registered for 20 bilayers were $\mathrm{FePc}^{\mathrm{SO} 3} / \mathrm{PAH}$ : 0.031, $\mathrm{NiPc}^{\mathrm{SO} 3} / \mathrm{PAH}$ : 0.158 and $\mathrm{CuPc}^{\mathrm{SO} 3} / \mathrm{PAH}: 0.189$ ). Taking into account that the molar extinction coefficients of the three phthalocyanines are of the same order of magnitude, it could be concluded that the $\mathrm{CuPc}^{\mathrm{SO} 3} / \mathrm{PAH}$ films were more closely packed than $\mathrm{NiPc}^{\mathrm{SO}} / \mathrm{PAH}$ films or $\mathrm{FePc}^{\mathrm{SO} 3} / \mathrm{PAH}$ films. The preparation method was highly reproducible and coefficients of variation calculated from the maximum absorbance of 20 bilayer films, were lower than $2 \%$. 


\subsection{EQCMMeasurements in Glucose and Catechol}

In a first approach and in order to test the sensing performance of the EQCM LbL films, they were immersed in catechol (an antioxidant usually found in grape juices) and glucose, one of the major sugars. Cyclic voltammograms (potential range from -1.0 to $+1.0 \mathrm{~V} v s$. $\mathrm{Ag} \mid \mathrm{AgCl}$ ) and massograms were recorded simultaneously.

The responses towards catechol are illustrated in Figure 2 for $\mathrm{NiPc}^{\mathrm{SO}} / \mathrm{PAH}$ films. It is important to remark that in all cases, the first scan was always different from the subsequent cycles. After the second cycle, scans were highly reproducible with a coefficient of variation $(\% \mathrm{CV})$ of the highest peak were lower than $2 \%$.

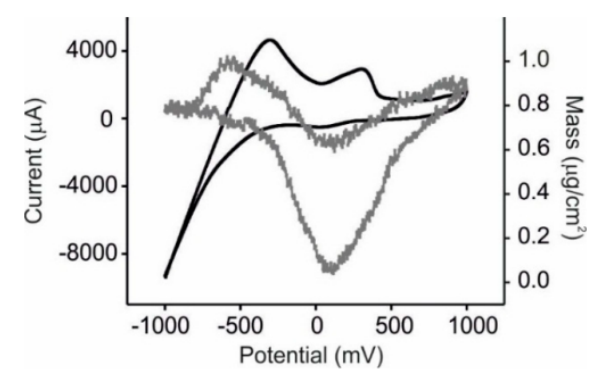

Figure 2. Response of the array of sensors towards catechol $10^{-3} \mathrm{~mol} \cdot \mathrm{L}^{-1}$ in $\mathrm{KCl}$ $0.1 \mathrm{~mol} \cdot \mathrm{L}^{-1}$. Voltammetric output (black line) and mass output (grey line) for the $\mathrm{NiPc}^{\mathrm{SO}} / \mathrm{PAH}$ sensor.

The voltammetric responses were characterized by two redox process, one corresponding to the oxidation/reduction of catechol (at $+0.30 \mathrm{~V}$ and $+0.05 \mathrm{~V}$ for the anodic and cathodic waves respectively). The decomposition of water occurring at negative potentials was accompanied by the oxidation of hydrogen that was observed as an anodic wave at $-0.45 \mathrm{~V}$. The four electrodes forming the array showed similar trends but the peak positions and their intensities differ from one electrode to another. For instance, the oxidation of catechol occurs at $+0.25 \mathrm{~V}$ for $\mathrm{FePc}^{\mathrm{SO}} / \mathrm{PAH},+0.30 \mathrm{~V}$ for $\mathrm{NiPc}^{\mathrm{SO}} / \mathrm{PAH}$ and $+0.42 \mathrm{~V}$ for $\mathrm{CuPc}^{\mathrm{SO} 3} / \mathrm{PAH}$ sensor, indicating that the electrocatalytic effect of the phthalocyanine decreased when advancing in the transition metal series. The intensity of the peaks increased with respect to the values observed in the bare $\mathrm{Pt}$ electrode $(2780 \mu \mathrm{A}), \mathrm{FePc}{ }^{\mathrm{SO}} / \mathrm{PAH}$ $(2800 \mu \mathrm{A}), \mathrm{NiPc}^{\mathrm{SO} 3} / \mathrm{PAH}(2930 \mu \mathrm{A})$ and $\mathrm{CuPc}^{\mathrm{SO} 3} / \mathrm{PAH}(3260 \mu \mathrm{A})$.

Hydrogen was formed during the water decomposition that occurred at ca. $-0.45 \mathrm{~V}$ according to the next reaction:

$$
2 \mathrm{H}_{2} \mathrm{O}+2 e^{-} \longrightarrow \mathrm{H}_{2}+2 \mathrm{OH}^{-}
$$

Also in this case, the peak position shifted to more negative values when advancing in the transition metal series $\left(-0.25 \mathrm{~V}\right.$ for $\mathrm{FePc}^{\mathrm{SO} 3} / \mathrm{PAH},-0.35 \mathrm{~V}$ for $\mathrm{NiPc}^{\mathrm{SO} 3} / \mathrm{PAH}$ and -0.42 for $\mathrm{CuPc}^{\mathrm{SO} 3} / \mathrm{PAH}$ sensor and -0.45 for the Pt bare electrode), confirming the electrocatalytic effect of the nanostructured films.

Massograms showed a mass increase during the oxidation of catechol and a large decrease in mass during the reduction. Massograms were highly stable, however, a net increase in the mass at the end of 
the experiment was observed, indicating that catechol was adhering to the surface of the electrode. This result is in good accordance with previously published results that demonstrated the passivation of the electrodes during cycling due to polymerization of phenols [58]. In fact, when sensors used to analyze catechol were immersed in a $\mathrm{KCl} 0.1 \mathrm{~mol} \cdot \mathrm{L}^{-1}$ solution, the presence of catechol was still observed. According to this, sensors were considered as single use devices and a brand new sensor must be prepared to measure each sample.

As expected, the glucose solution did not produce redox peaks in the studied range (except peaks associated to protons at negative potentials). A progressive increase in mass was observed under consecutive cycling that could be attributed to the adhesion of sugars to the sensing surface. The effect of the concentration in the responses of the sensors was analyzed by exposing the EQCM devices to different catechol and glucose concentrations. The results are illustrated in Figure 3 for $\mathrm{CuPc}^{\mathrm{SO}} / \mathrm{PAH}$. When increasing the concentration of catechol, the voltammetric peak at $-0.4 \mathrm{~V}$ decreased in intensity, while peak at ca. +0.4 increased in intensity and simultaneously shifts to lower potentials. Similarly, the shape of massograms was different depending on the concentration. This behavior impedes the construction of calibration curves and justifies the need of an array of electrodes, where the whole curve provides information about the sample.
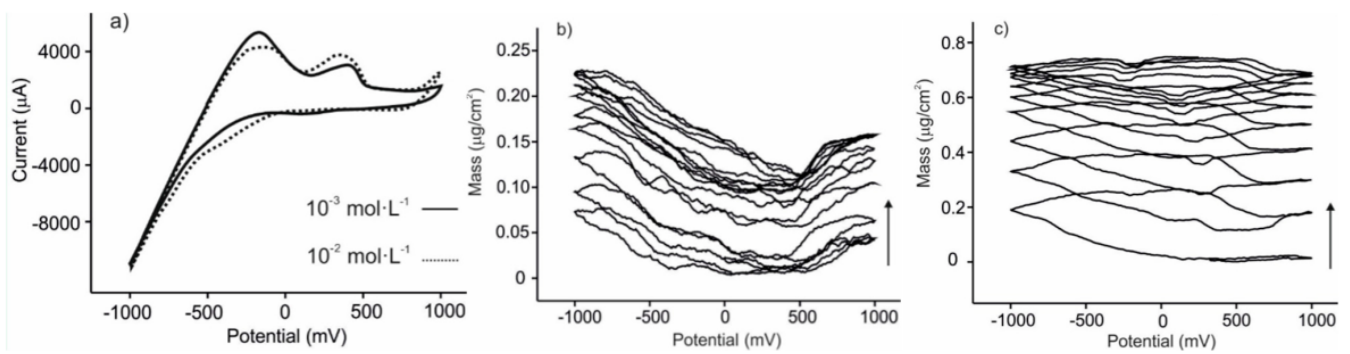

Figure 3. (a) Voltammetric response towards catechol in $\mathrm{KCl} 0.1 \mathrm{~mol} \cdot \mathrm{L}^{-1}$ for the $\mathrm{CuPc}^{\mathrm{SO} 3} / \mathrm{PAH}$ sensor; (b) Mass response of the $\mathrm{CuPc}^{\mathrm{SO} 3} / \mathrm{PAH}$ sensor towards glucose $10^{-3} \mathrm{~mol} \cdot \mathrm{L}^{-1}$ in $0.01 \mathrm{~mol} \cdot \mathrm{L}^{-1}$ phosphate buffer ( $\mathrm{pH} 7.0$ ); (c) Mass response of the $\mathrm{CuPc}^{\mathrm{SO}} / \mathrm{PAH}$ sensor towards glucose $10^{-2} \mathrm{~mol} \cdot \mathrm{L}^{-1}$ in $0.01 \mathrm{~mol} \cdot \mathrm{L}^{-1}$ phosphate buffer $(\mathrm{pH} 7.0)$.

\subsection{Analysis of Grape Juices}

The array of sensors was immersed in must samples prepared from different varieties of grape and EQCM analysis was performed. It has to be pointed out that preliminary experiments were carried out in undiluted must and the signals demonstrated a poor reproducibility. This can be attributed to the large amount of suspended materials (i.e. proteins) present in must samples that can adhere to the sensor surface. The reproducibility was clearly improved when musts were diluted $1: 2$ in $\mathrm{KCl}$ $0.05 \mathrm{~mol} \cdot \mathrm{L}^{-1}$. For this reason, further experiments were carried out in diluted musts. Cyclic voltammetry was carried out at a potential range from -0.6 to $+1.0 \mathrm{~V}$ vs. $\mathrm{Ag} \mid \mathrm{AgCl}$ and voltammograms/massograms were simultaneously registered. As an example of the responses obtained Figure 4 shows the voltammetric and mass outputs of $\mathrm{NiPc}^{\mathrm{SO}} / \mathrm{PAH}$ sensor towards a must obtained from the Juan García grape variety. 


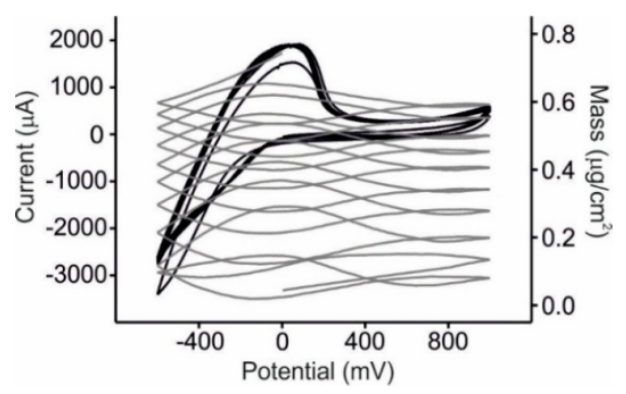

Figure 4. Voltammetric (black line) and mass (grey line) response of the $\mathrm{NiPc}^{\mathrm{SO} 3} / \mathrm{PAH}$ sensor towards a must obtained from Juan García grapes.

As usual, the first cycle was different from the rest, but subsequent responses were stable and highly reproducible with coefficients of variation $(\% \mathrm{CV})$ within the range of $0.4 \%-2.8 \%$. Voltammograms were dominated by a broad anodic peak that could be associated to the polyphenolic content of wines $[8,59]$. On the other hand, the massograms showed a progressive increase in mass. In a complex mixture such as must, it is difficult to establish the process by which the mass increases. Taken into account the results obtained in the presence of catechol and glucose, it can be assumed that polymerization of phenols can play a key role in the observed mass changes. In addition, the adhesion of other main components of musts without redox activity in the studied range such as sugars cannot be neglected.

The results obtained were similar for all the phthalocyanines tested, but the peak positions and their intensities depend on the phthalocyanine used to form the LbL film. Figure 5 illustrates the voltammetric response of the array of sensors towards musts. The responses obtained towards the Mencía Regadío and the Juan García varieties have been chosen as examples. In good accordance with the results obtained in catechol, the peak positions shifted to higher values when advancing in the transition metal series, confirming that these anodic peaks are associated to the polyphenolic content of grapes.

Because the polyphenolic content varied from one must to another (see Table 1), the positions, broadness and intensities of the peaks were different.

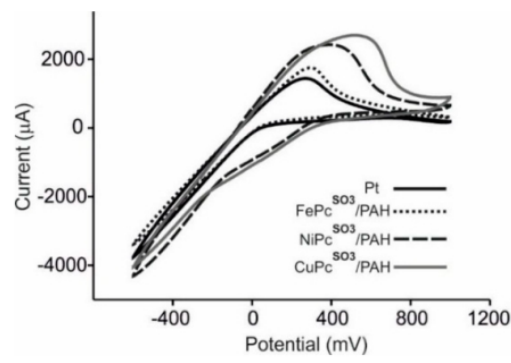

(a)

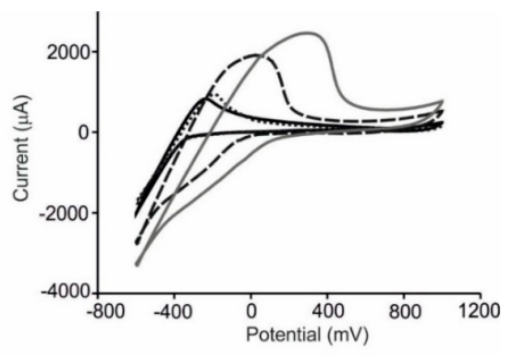

(b)

Figure 5. Voltammetric outputs of the EQCM sensors immersed in (a) Mencía Regadío; (b) Juan García grapes. 
The massograms registered simultaneously to the cyclic voltammetry are illustrated in Figure 6 for different sensors/musts (due to the complexity of the signals it is not possible to superimpose signals for comparison purposes). As expected, all massograms showed a progressive increase in mass, but also in this case, each must produce a different signature. The change in mass could be attributed to the adhesion of sugars and/or to other complex processes and polymerizations. It has to be noticed that in some cases, the adhesion of suspended material caused spikes or a sudden increase in mass. In those cases (15\% of the measures), the results were discarded. In the absence of such processes, the experiments were highly reproducible.

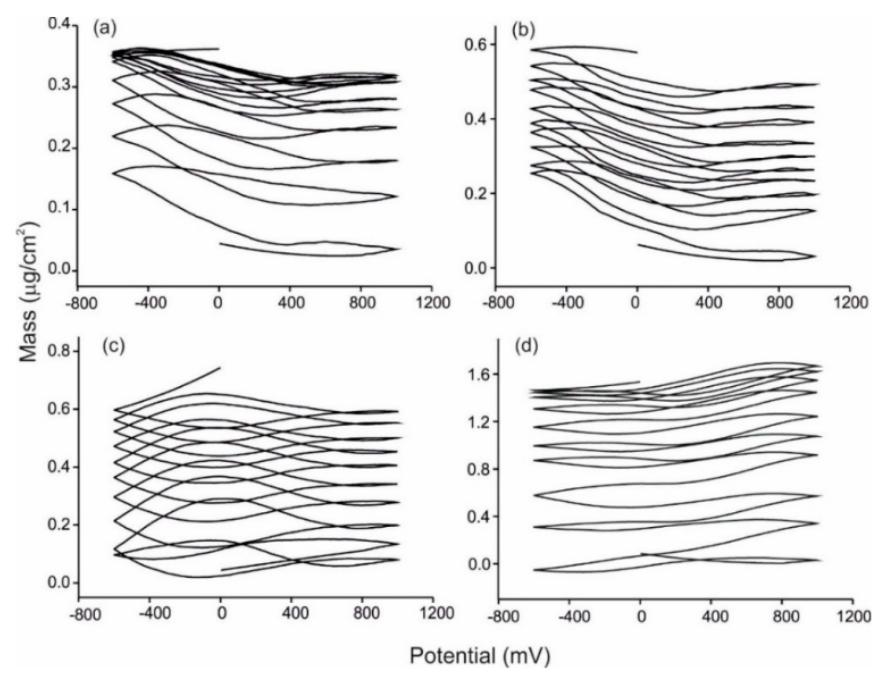

Figure 6. Mass outputs of (a) Pt bare sensor immersed in Prieto Picudo; (b) $\mathrm{FePc}^{\mathrm{SO} 3} / \mathrm{PAH}$ sensor immersed in Tempranillo; (c) $\mathrm{NiPc}^{\mathrm{SO} 3} / \mathrm{PAH}$ sensor immersed in Juan García; (d) $\mathrm{CuPc}^{\mathrm{SO} 3} / \mathrm{PAH}$ sensor immersed in Cabernet.

\subsection{Statistical Analysis}

The intrinsic complexity, richness and cross-selectivity of the signals generated by voltammetric sensor arrays are an advantage because the dataset contains meaningful information about the sample. As the array of EQCM sensors showed a characteristic voltammetric and mass response depending on the must analyzed, these data could be used to discriminate musts according to their chemical nature In order to evaluate the capability of discrimination of the system, a non-supervised multivariate technique, the multi-way decomposition PARAFAC method was used.

Prior to statistical analysis, voltammograms and massograms were preprocessed by means of a data reduction technique using "kernels" to obtain 10 variables from each voltammogram and 10 variables from each massogram. The input array for computing the PARAFAC model was a three-way data matrix ("6 must samples with 3 replicas" $\times$ " 10 kernels" $\times$ " 4 sensors"), therefore the size was $(18 \times 10 \times 4)$.

Figure 7 represents the tridimensional PARAFAC scores plot for the multisensor system using voltammetric outputs. As observed in the figure, the clusters corresponding to the six studied musts 
(three replicas per must) were clearly separated and were located according to the Total Polyphenol Index (TPI): musts with high TPI (Mencía Regadío, Cabernet Sauvignon, Tempranillo and Juan García variety) appeared in the region corresponding to a positive C3, while musts with low TPI (Prieto Picudo and Garnacha variety) appeared in the negative C3 values region. This good discrimination can be explained taking into account that voltammograms reflect the redox activity of the phenols. The error in terms of root-mean-square error (RMSE) was 0.4551.

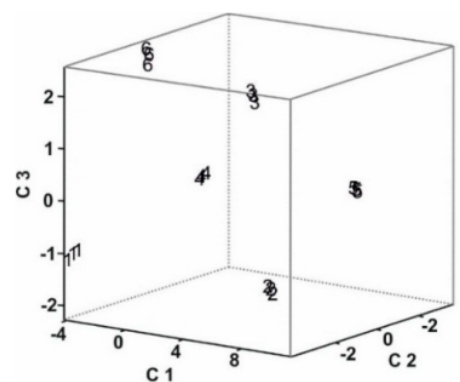

(a)

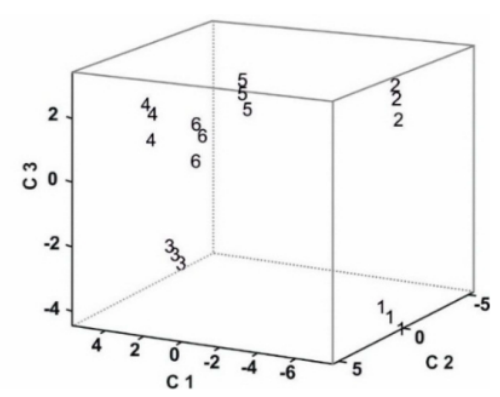

(b)

Figure 7. PARAFAC scores plot of the array obtained from (a) The voltammetric responses; (b) The mass responses. Must samples are 1: Prieto Picudo; 2: Garnacha; 3: Cabernet Saugvinon; 4: Tempranillo; 5: Juan García; and 6: Mencía Regadío.

Figure 7 also shows the tridimensional PARAFAC scores plot when analyzing the mass output. In this case RMSE was 0.6472 . Massograms also allowed a good-quality discrimination. However, the positions of the clusters were not related to the Total Polyphenol Index. Instead, their positions were located according to the sugar content.

Musts with the highest Brix degree (Cabernet Sauvignon and Prieto Picudo) were located at negative values of $\mathrm{C} 3$, whereas those with lower sugar content at positive values of C3 (Tempranillo, Mencía Regadío, Juan García and Garnacha). This result confirms that changes in mass observed from one massogram to the subsequent one are due to the adhesion of sugars.

Prediction models were established by N-PLS. The classification models were subjected to full cross-validation by means of the "leave-one-out" method. N-PLS was carried out to establish correlations between the EQCM signals obtained from the array and chemical parameters. N-PLS regression builds a calibration model incorporating a relationship between the sets of predictors and responses based on the multiway structure of the arrays. Calibration fits the model to the available data, while validation checks the model for new data. Results are shown in Tables 2 and 3.

Correlations were obtained with the voltammetric data, which showed higher coefficients of correlations and lower errors. Results indicate that the voltammetric signals are better correlated with the TPI with only three components (Table 2).

The mass outputs present a better correlation with those parameters related to the sugar content (sugar content, degree 16.8 and Brix degrees) (Table 3) than with those related to the polyphenolic content. The correlations calculated between mass data and chemical parameters showed lower correlation coefficients and higher errors than the correlations obtained with voltammetric data. 
Table 2. Statistical parameters obtained for the N-PLS regression model established between the chemical parameters and the voltammetric responses of the sensors towards musts.

\begin{tabular}{|c|c|c|c|c|c|}
\hline \multicolumn{6}{|c|}{ Voltammetric Outputs } \\
\hline Parameters & $\mathrm{R}^{2}{ }_{\mathrm{C}}^{\text {(a) }}$ & RMSEC $^{(b)}$ & $\mathrm{R}_{\mathrm{P}}^{2}(\mathrm{c})$ & RMSEP (d) & Number of Components \\
\hline Sugar content & 0.997 & 0.99187 & 0.945 & 4.24917 & 4 \\
\hline Brix degree & 0.996 & 0.09242 & 0.935 & 0.40019 & 4 \\
\hline Degree 16.8 & 0.997 & 0.05894 & 0.946 & 0.25147 & 4 \\
\hline TPI & 0.992 & 0.46538 & 0.983 & 0.68089 & 3 \\
\hline $\begin{array}{l}\text { olyphenolic content } \\
\text { in-Ciocalteau method }\end{array}$ & 0.998 & 0.33442 & 0.989 & 1.11841 & 3 \\
\hline
\end{tabular}

(a) Squared correlation coefficient in calibration; (b) Root Mean Square Error of Calibration; (c) Squared correlation coefficient in prediction; (d) Root Mean Square Error of Prediction.

Table 3. Statistical parameters obtained for the N-PLS regression model established between the chemical parameters and the mass responses of the sensors towards musts.

\begin{tabular}{|c|c|c|c|c|c|}
\hline \multicolumn{6}{|c|}{ Mass Outputs } \\
\hline Parameters & $\mathrm{R}^{2}{ }_{\mathrm{C}}^{\text {(a) }}$ & RMSEC $^{(b)}$ & $R_{P}^{2}(c)$ & RMSEP (d) & Number of Components \\
\hline Sugar content & 0.941 & 4.45005 & 0.839 & 7.31293 & 4 \\
\hline Brix degree & 0.972 & 0.00176 & 0.865 & 0.00291 & 4 \\
\hline Degree 16.8 & 0.941 & 0.26438 & 0.840 & 0.43420 & 4 \\
\hline TPI & 0.961 & 1.02511 & 0.845 & 2.04940 & 5 \\
\hline $\begin{array}{l}\text { Polyphenolic content } \\
\text { Folin-Ciocalteau method }\end{array}$ & 0.965 & 1.91428 & 0.921 & 3.0353 & 5 \\
\hline
\end{tabular}

As voltammetric and mass data are registered simultaneously, EQCM can improve the discrimination of must samples while providing good correlations with both sugar and polyhenolic content.

\section{Conclusions}

A multisensor system formed by EQCM sensors modified with LbL films containing phthalocyanines have been successfully fabricated and used to discriminate musts obtained from different varieties of grapes. The capability of the sensor array to discriminate grapes according to their chemical parameters has been evidenced using the multivariate decomposition method PARAFAC. Voltammograms showed anodic peaks that were related to the phenolic content in musts and massograms showed an increase of mass that was related to the sugar content of the musts. Prediction models performed by multi-way partial least squares (N-PLS) have showed correlations between the voltammetric and mass outputs with the chemical parameters. EQCM has demonstrated to be advantageous because voltammetric and mass data can be registered simultaneously, providing good correlations with the sugar and the polyhenolic content, which are the most important indicators of quality and degree of ripeness in grapes. 


\section{Acknowledgments}

Financial support by MINECO and FEDER (grant CICYT-AGL2012-33535) and the Junta de Castilla y León (VA-032U13) is gratefully acknowledged. Cristina Medina-Plaza would also like to thank the University of Valladolid for a grant (FPI-UVA).

\section{Author Contributions}

The manuscript was written by Maria Luz Rodríguez-Méndez and Celia Garcia-Hernandez. In addition, the concept of the experimental setup was also proposed by Maria Luz Rodriguez-Méndez and implemented by Celia Garcia-Hernandez. For the experimental works, the sensing tests and material analyses were done by Celia Garcia-Hernandez, Cristina Medina-Plaza, Cristina Garcia-Cabezon, Isabel del Valle and Jose Antonio de Saja. Finally, data analyses were accomplished by Celia Garcia-Hernandez and Fernando Martin-Pedrosa. The final manuscript has been read and approved by all authors.

\section{Conflicts of Interest}

The authors declare no conflict of interest.

\section{References}

1. Tahara, Y.; Toko, K. Electronic tongues-A Review. IEEE Sens. J. 2013, 13, 3001-3011.

2. Śliwińska, M.; Wiśniewska, P.; Dymerski, T.; Namieśnik, J.; Wardencki, W. Food analysis using artificial senses. J. Agric. Food Chem. 2014, 62, 1423-1448.

3. Kirsanov, D.; Mednova, O.; Vietoris, V.; Kilmartin, P.A.; Legin, A. Towards reliable estimation of an "electronic tongue" predictive ability from PLS regression models in wine analysis. Talanta 2012, $90,109-116$.

4. Oliveri, P.; Casolino, C.; Forina, M. Chemometric Brains for Artificial Tongues. In Advances in Food and Nutrition Research; Elsevier Inc.: Amsterdam, The Netherlands, 2010; pp. 57-117.

5. Ciosek, P.; Wroblewski, W. Potentiometric Electronic Tongues for Foodstuff and Biosample Recognition-An Overview. Sensors 2011, 11, 4688-4701.

6. Scampicchio, M.; Ballabio, D.; Arecchi, A.; Cosio, S.M.; Mannino, S. Amperometric electronic tongue for food analysis. Microchim. Acta 2008, 163, 11-21.

7. Winquist, F.; Olsson, J.; Eriksson, M. Multicomponent analysis of drinking water by a voltammetric electronic tongue. Anal. Chim. Acta 2011, 683, 192-197.

8. Parra, V.; Arrieta, A.; Fernández-Escudero, J.A.; Rodríguez-Méndez, M.L.; de Saja, J.A. Electronic tongue based on chemically modified electrodes and voltammetry for the detection of adulterations in wines. Sens. Actuators B 2006, 118, 448-453

9. Ceto, X.; Apetrei, C.; del Valle, M.; Rodriguez-Mendez, M.L. Evaluation of red wines antioxidant capacity by means of a voltammetric e-tongue with an optimized sensor array. Electrochim. Acta 2014, 120, 180-186.

10. Riul, A.; Dantas, C.A.R.; Miyazaki, C.M.; Oliveira, O.N. Recent advances in electronic tongues. Analyst 2010, 135, 2481-2495. 
11. Gil-Sanchez, L.; Soto, J.; Martinez-Mañez, R.; Garcia-Breijo, E.; Ibañez, J.; Llobet, E. A novel humid electronic nose combined with an electronic tongue for assessing deterioration of wine. Sens. Actuators A 2011, 171, 152-158.

12. Buratti, S.; Ballabio, D.; Benedetti, S.; Cosio, M.S. Prediction of Italian red wine sensorial descriptors from electronic nose, electronic tongue and spectrophotometric measurements by means of genetic algorithm regression models. Food Chem. 2007, 100, 211-218.

13. Di Natale, C.; Paolesse, R.; Macagnano, A.; Mantini, A.; D’Amico, A.; Ubigli, M.; Legin, A.; Lvova, L.; Rudnitskaya, A.; Vlasov, Y. Application of a combined artificial olfaction and taste system to the quantification of relevant compounds in red wine. Sens. Actuators B 2000, 69, 342-347.

14. Rudnitskaya, A.; Rocha, S.M.; Legin, A.; Pereira, V.; Marques, J.C. Evaluation of the feasibility of the electronic tongue as a rapid analytical tool for wine age prediction and quantification of the organic acids and phenolic compounds. The case-study of Madeira wine. Anal. Chim. Acta 2010, $662,82-89$.

15. Parra, V.; Hernando, T.; Rodriguez-Mendez, M.L.; de Saja, J.A. Electrochemical sensor array made from bisphthalocyanine modified carbon paste electrodes for discrimination of red wines. Electrochim. Acta 2004, 49, 5177-5185.

16. Prieto, N.; Gay, M.; Vidal, S.; Aagaard, O.; de Saja, J.A.; Rodriguez-Mendez, M.L. Analysis of the influence of the type of closure in the organoleptic characteristics of a red wine by using an electronic panel. Food Chem. 2011, 129, 589-594.

17. Parra, V.; Arrieta, A.; Fernandez-Escudero, J.A.; Garcia, H.; Apetrei, C.; Rodriguez-Mendez, M.L.; de Saja, J.A. E-tongue based on a hybrid array of voltammetric sensors based on phthalocyanines, perylene derivatives and conducting polymers: Discrimination capability towards red wines elaborated with different variety of grapes. Sens. Actuators B 2006, 115, 54-61.

18. Apetrei, C.; Apetrei, I.; Nevares, I.; del Alamo, M.; Parra, V.; Rodríguez-Méndez, M.L.; de Saja, J.A. Using an e-tongue based on voltammetric electrodes to discriminate among red wines aged in oak barrels or aged using alternative methods. Correlation between electrochemical signals and analytical parameters. Electrochim. Acta 2007, 52, 2588-2594.

19. Legin, A.; Rudnitskaya, A.; Lvova, L.; Vlasov, Y.; di Natale, C.; D’Amico, A. Evaluation of Italian wine by the electronic tongue:recognition, quantitative analysis and correlation with human sensory perception. Anal. Chim. Acta 2003, 484, 33-44.

20. Baldacci, S.; Matsuno, T.; Toko, K.; Stella, R.; de Rossi, D. Discrimination of wine using taste and smell sensors. Sens. Mater. 1998, 10, 185-200.

21. Rodriguez-Mendez, M.L.; Arrieta, A.A.; Parra, V.; Bernal, A.; Vegas, A.; Villanueva, S.; Gutierrez-Osuna, R.; de Saja, J.A. Fusion of three sensory modalities for the multimodal characterization of red wines. IEEE Sens. J. 2004, 4, 348-354.

22. Lvova, L.; Pudi, R.; Galloni, P.; Lippolis, V.; di Natale, C.; Lundstrom, I.; Paolesse, R. Multi-transduction sensing films for Electronic Tongue applications. Sens. Actuators B 2015, 207 , 1076-1086.

23. Gutierrez, M.; Llobera, A.; Vila-Planas, J.; Capdevila, F.; Demming, S.; Büttgenbach, S.; Mínguez, S.; Jiménez-Jorquera, C. Hybrid electronic tongue based on optical and electrochemical microsensors for quality control of wine. Analyst 2010, 135, 1718-1725. 
24. Apetrei, I.M.; Rodriguez-Mendez, M.L.; Apetrei, C.; Nevares, I.; del Alamo, M.; de Saja, J.A. Monitoring of evolution during red wine aging in oak barrels and alternative method by means of an electronic panel test. Food Res. Int. 2012, 45, 244-249.

25. Zampetti, E.; Pantalei, S.; Macagnano, A.; Proeitti, E.; di Natale, C.; D'Amico, A. Use of multiplexed oscillator in a miniaturized electronic nose based on a multichannel quartz crystal microbalance. Sens. Actuators B 2008, 131, 159-166.

26. Compagnone, D.; Faieta, M.; Pizzoni, D.; di Natale, C.; Paolesse, R.; van Caelenberg, T.; Beheydt, B.; Pittia, P. Quartz crystal microbalance gas sensor arrays for the quality control of chocolate. Sens. Actuators B 2015, 207, 1114-1120.

27. Wilson, A.D.; Baietto, M. Advances in electronic-nose technologies developed for biomedical applications. Sensors 2011, 11, 1105-1176.

28. Panigrahi, S.; Sankaran, S.; Mallik, S.; Gaddam, B.; Hanson, A.A. Olfactory receptor-based polypeptide sensor for acetic acid VOC detection. Mater. Sci. Eng. C 2012, 32, 1307-1313.

29. Guo, X.; Lin, C.S.; Chen, S.H.; Ye, R.; Wu, V.C.H. A piezoelectric immunosensor for specific capture and enrichment of viable pathogens by quartz crystal microbalance sensor, followed by detection with antibody-functionalized gold nanoparticles. Biosens. Bioelectron. 2012, 38 , 177-183.

30. Ermolaeva, T.N.; Kalmykova, E.N.; Shashkanova, O.Y. Piezoquartz biosensors for the analysis of environmental objects, foodstuff and for clinical diagnostic. Russ. J. Gen. Chem. 2008, 78, 2430-2444.

31. Sun, H.; Mo, Z.H.; Choy, J.T.S.; Zhu, D.R., Fung, Y.S. Piezoelectric quartz crystal sensor for sensing taste-causing compounds in food. Sens. Actuators B 2008, 131, 148-158.

32. Hauptmann, P.; Borngraeber, R.; Schroeder, J.; Auge, J. Application of novel sensor electronics for quartz resonators in artificial tongue. In Proceedings of the 2000 IEEE/EIA International Frequency Control Symposium \& Exhibition, Kansas City, MO, USA, 7-9 June 2000; pp. 100-105.

33. Gay-Martin, M.; Diez-Arevalo, E.; Rodriguez-Mendez, M.L.; Saja, J.A.D. Electrochemical quartz crystal microbalance analysis of the oxidation reaction of phenols found in wines at lutetium bisphthalocyanine electrodes. Sens. Actuators B 2013, 185, 24-31.

34. Cheng, C.I.; Chang, Y.P.; Chu, Y.H. Biomolecular interactions and tools for their recognition: Focus on the quartz crystal microbalance and its diverse surface chemistries and applications. Chem. Soc. Rev. 2012, 41, 1947-1971.

35. Medina-Plaza, C.; De Saja J.A.; Rodriguez-Mendez, M.L. Bioelectronic tongue based on lipidic nanostructured layers containing phenol oxidases and lutetium bisphthalocyanine for the analysis of grapes. Biosens. Bioelectron. 2014, 57, 276-283.

36. Ozmen, M.; Ozbek, Z.; Bayrakci, M.; Ertul, S.; Ersoz, M.; Capan, R. Preparation and gas sensing properties of Langmuir-Blodgett thin films of calix[n]arenes: Investigation of cavity effect. Sens. Actuators B 2014, 195, 156-164.

37. Deligöz, H.; Tieke, B. QCM-D study of layer by layer assembly of polyelectrolyte blend films and their drug loading-release behavior. Colloids Surf. A 2014, 441, 725-736. 
38. Erbahar, D.D.; Harbeck, M.; Gumus, G.; Gurol, I.; Ahsen, V. Self-assembly of phthalocyanines on quartz crystal microbalances for QCM liquid sensing applications. Sens. Actuators B 2014, $190,651-656$.

39. Ningsih, P.; Holdsworth, C.Z.; Donne, S.W. Mn-modified polypyrrole thin films for supercapacitor electrodes. Synth. Meth. 2014, 196, 8-19.

40. Martin, M.G.; de Saja, J.A.; Munoz, R.; Rodriguez-Mendez, M.L. Multisensor system based on bisphthalocyanine nanowires for the detection of antioxidants. Electrochim. Acta 2012, 68, 88-94.

41. Wang, M.H.; Liu, S.L.; Zhang, Y.C.; Yang, Y.Q.; Shi, Y.; He, L.H.; Fang, S.M.; Zhang, Z.H. Graphene nanostructures with plasma polymerized allylamine biosensor for selective detection of mercury ions. Sens. Actuators B 2014, 203, 497-503.

42. Jha, S.K.; Liu, C.J.; Hayashi, K. Molecular imprinted polyacrylic acids based QCM sensor array for recognition of organic acids in body odor. Sens. Actuators B 2014, 204, 74-87.

43. Su, Y.H.; Xie, Q.J.; Chen, C.; Zhang, Q.F.; Ma, M.; Yao, S.Z. Electrochemical quartz crystal microbalance studies on enzymatic specific activity and direct electrochemistry of immobilized glucose oxidase in the presence of sodium dodecyl benzene sulfonate and multiwalled carbon nanotubes. Biotechnol. Prog. 2008, 24, 262-272.

44. Yu, R.M.; Ma, M.; Wang, L.L.; Xie, Q.J.; Cao, Z.J.; Jiang, X.Q.; Yao, S.Z. Highly sensitive and surface-renewable electrochemical quartz crystal microbalance assays of heparin and chondroitin sulfate based on their effects on the electrodeposition of neutral red. Biosens. Bioelectron. 2009, 24, 1771-1776.

45. Subbaiyan, N.K.; Obraztsov, L.; Wijesinghe, C.A.; Tran, K.; Kutner, W.; D'Souza, F. Supramolecular Donor-Acceptor Hybrid of Electropolymerized Zinc Porphyrin with Axially Coordinated Fullerene: Formation, Characterization, and Photoelectrochemical Properties. J. Phys. Chem. C 2009, 113, 8982-8989.

46. Sigalov, S.; Levi, M.D.; Salitra, G.; Aurbach, D. EQCM as a unique tool for determination of ionic fluxes in microporous carbons as a function of surface charge distribution. $J$. Electrochem. Commun. 2010, 12, 1718-1721.

47. Malitesta, C.; Picca, R.A.; Mazzotta, E.; Guascito, M.R. Tools for the development of electrochemical sensors: An EQCM flow cell with flow focusing. Electroanal 2012, 24, 790-797.

48. Pinto, E.M.; Gouveia-Caridade, C.; Soares, D.M.; Brett, C.M.A. Electrochemical and surface characterisation of carbon-film-coated piezoelectric quartz crystals. Appl. Surf. Sci. 2009, 255, 8084-8090

49. Brown, R.J.C.; Brett, D.J.L.; Kucernak, A.R.J. An electrochemical quartz crystal microbalance study of platinum phthalocyanine thin films. J. Electroanal. Chem. 2009, 633, 339-346.

50. Harbeck, M.; Erbahar, D.D.; Gurol, I.; Musluoglu, E.; Ahsen, V.; Ozturk, Z.Z. Phthalocyanines as sensitive coatings for QCM sensors operating in liquids for the detection of organic compounds. Sens. Actuators B 2010, 150, 346-354.

51. Rodriguez-Mendez, M.L.; Gay, M.; de Saja, J.A. New insights into sensors based on radical bisphthalocyanines. J. Porphy. Phthalocyan. 2009, 13, 1159-1167.

52. Alessio, P.; Pavinatto, F.J.; Oliveira, O.N.; Saez, J.A.D.; Constantino, C.J.L.; Rodriguez-Mendez, M.L. Detection of catechol using mixed Langmuir-Blodgett films of a phospholipid and phthalocyanines as voltammetric sensors. Analyst 2010, 135, 2591-2599. 
53. Volpati, D.; Alessio, P.; Zanfolim, A.A.; Storti, F.C.; Job, A.E.; Ferreira, M.; Riul, A.; Oliveira, O.N.; Constantino, C.J.L. Exploiting distinct molecular architectures of ultrathin films made with iron phthalocyanine for sensing. J. Phys. Chem. B 2008, 112, 15275-15282.

54. Zucolotto, V.; Ferreira, M.; Cordeiro, M.R.; Constantino, C.J.L.; Balogh, D.T.; Zanatta, A.R.; Moreira, W.C.; Oliveira, O.N. Unusual interactions binding iron tetrasulfonated phthalocyanine and poly(allylamine hydrochloride) in layer-by-layer films. J. Phys. Chem. B 2003, 107, 3733-3737.

55. OIV. Compendium of International Methods of Analysis of Wines and Musts; Organisation Internationale de la Vigneet du Vin: Paris, France, 2013.

56. Aoki, P.H.B.; Volpati, D.; Riul, A.; Caetano, W.; Constantino, C.J.L. Layer-by-Layer technique as a new approach to produce nanostructured films containing phospholipids as transducers in sensing applications. Langmuir 2009, 25, 2331-2338.

57. Gutiérrez-Osuna, R.; Nagle, H.T. A method for evaluating data-preprocessing techniques for odor classification with an array of gas sensors. IEEE Trans. Syst. Man Cybern. B 1999, 29, 626-632.

58. Ferreira, M.; Varela, H.; Torresi, R.M.; Tremiliosi, G. Electrode passivation caused by polymerization of different phenolic compounds. Electrochim. Acta 2006, 52, 434-442.

59. Rodriguez-Mendez, M.L.; Apetrei, C.; Gay, M.; Medina-Plaza, C.; de Saja, J.A.; Vidal, S.; Aagaard, O.; Ugliano, M.; Wirth, J.; Cheynier, V. Evaluation of oxygen exposure levels and polyphenolic content of red wines using an electronic panel formed by an electronic nose and an electronic tongue. Food Chem. 2014, 155, 91-97.

(C) 2015 by the authors; licensee MDPI, Basel, Switzerland. This article is an open access article distributed under the terms and conditions of the Creative Commons Attribution license (http://creativecommons.org/licenses/by/4.0/). 



\subsection{Paper IX}

In situ synthesis, stabilization and activity of protein-modified gold nanoparticles for biological applications.

By Celia García-Hernández, Allison Freese, María L. Rodríguez-Méndez and Adam K. Wanekaya.

Biomaterials Science 7 (2019) 2511-2519.

(doi: 10.1039/c9bm00129h) 


\subsubsection{Motivation and objective}

This work was carried out in the Missouri State University, in the Chemistry Department, under the supervision of Prof. Wanekaya during an international research stay. It has a different topic, not related to electronic tongue devices, but the LbL technique has also been used in this work to encapsulate the protein lysozyme for drug delivery purposes. It has, therefore, been included in this compilation of papers as it can be of interest for the developed bioelectronic tongues, where enzymes have improved the specificity of the electrochemical sensors. In previous works, the enzyme material was deposited onto the electrode surfaces by means of the drop-casting technique and, later, cross-linked with glutaraldehyde. Hence, the present work envisions a new methodology in which an enzyme can be bonded to AuNPs using stronger interactions, concretely, covalent bonds.

Nanomaterials applied to the field of delivering proteins and drugs into cells provide important advances for biomedical purposes. Most proposed methods for delivering proteins are based on mechanical methods, covalent protein modification and supramolecular delivery systems. All these approaches present different strengths and challenges. The Layer by Layer (LbL) technique has gained great interest in protein delivery and biosensor applications. LbL designs to immobilize or encapsulate enzymes provide a huge versatility and potential in the pharmaceutical and biopharmaceutical fields. Besides the advantages provided by LbL, such as ultrathin films, easy preparation, possibility to perform on supports of any shape and dimension, high versatility to surface modification with different polyelectrolytes, etc., in the field of protein immobilization, the use of mild conditions (e.g., aqueous solutions) is particularly advantageous in order to avoid the protein denaturation and protect the activity in comparison with the use of organic solvents used in other encapsulation systems (microparticles, microcapsules, microemulsions and liposomes). The most suitable polymers used for LbL designs are the positive charged polymers poly(ethyleneimine) (PEI) and poly(allylamine hydrochloride) (PAH) and the negative charged polymers poly(sodium 4-styrenesulfonate) (PSS) and poly[2(dimethylamino)ethyl methacrylate] (PDMAEMA). However, LbL multilayers have been successfully grown by means of polymers with a low charge density, so it has been demonstrated that the buildup of LbL films is not restricted to materials with a minimum charge density, since other driving interactions play an important role during the assembly. Moreover, when using purely electrostatic interactions, variations in the $\mathrm{pH}$, ionic strength, electrical field, light, 
temperature, mechanical stress, addition of biological compounds (e.g., proteins), or ionic surfactants might affect the properties of the adsorbed layers or even induce their desorption.

Gold nanoparticles (AuNPs) have attracted considerable interest because of their unique optical, electronic and catalytic properties. They have been widely used in chemical and biological applications due to their biocompatibility, dimension, easy characterization and the versatility offered by its surface chemistry. Gold nanoparticles can be synthesized using naturally occurring biomacromolecules instead of traditional methods that use reducing agents like borohydride and/or sodium citrate. However, some of these alternative methods use additional activators to initiate the reducing properties of the biomacromolecules. Amino acids, such as cysteine, are fine reducing agents and the biomaterials synthesized by using proteins are far more chemically pure and easy to characterize than by using different reducing and stabilizing agents in the presence of proteins.

In this work, we have developed a carrier system based on lysozyme and gold nanoparticles using the LbL technology. Lysozyme (Lys) is a natural enzyme abundantly found in secretions, such as tears, saliva, and mucus of the mammalian. The major function of Lys in vivo is against infection due to its antibacterial properties. Lysozyme has also been reported as an effective enzyme against many food spoilage and pathogenic microorganisms. However, the practical application of free lysozyme is limited because it is unstable and easily inactivated. Its relatively small size, low molecular weight $(14,370 \mathrm{kDa})$ and high isoelectric point (pl 11.35) allows an easier characterization and manipulation. In addition, lysozyme is stable over wide temperature and $\mathrm{pH}$ ranges, making it suitable for a variety of synthetic approaches. These properties also make this protein attractive to use as a model protein to develop specific carriers for small drug delivery.

\subsubsection{Summary of the research work}

The carrier systems based on Lys and AuNPs were prepared by following two different approaches. First, we envisioned a new strategy based on the use of the lysozyme as reducing agent to make AuNPs covalently bond with lysozyme, followed by the deposition of the PSS and PEI layers by means of LbL (PEI-PSS-Lys-AuNPS). PEI was used as the cationic layer and it was also deposited as the outer layer, since it plays an important role as the transfection agent and 
avoids the degradation of the protein. Secondly, colloidal citrate-stabilized AuNPs were functionalized with MUA (11-mercapto-undecanoic acid) and LbL was subsequently performed to deposit the PEI and PSS layers. In this case, the negatively charged polymer PSS was used to electrostatically immobilize the positive lysozyme in between two PSS layers. The resulting bioconjugate as-prepared was PEI-PSS-Lys-PSS-PEI-MUA-AuNPS.

The deposition of each layer was characterized following three important parameters: particle size, zeta potential, UV-Vis absorbance. In addition, the enzymatic activity was tested using the bacteria Micrococcus lysodeikticus as substrate.

In the case of the bioconjugate PEI-PSS-Lys-AuNPs, the lysozyme was mixed with a solution of $\mathrm{HAuCl}_{4}$ for 6 hours at $45^{\circ} \mathrm{C}$. Then, Lys acted as the reducing and capping agent of the AuNPs. During the reaction, the UV-Vis spectra showed a progressive increase in the Au Plasmon resonance peak after 4 hours of reaction. This peak reached its maximum at approximately 12 hours. The hydrodynamic diameters by DLS were found to be about $60 \mathrm{~nm}$ for Lys-AuNPs and PSS-Lys-AuNPs. The diameter increased to $90 \mathrm{~nm}$ after PEI deposition. The Zeta potential results evidenced the successful deposition of layers on the particle surface. Initially, before the coating, the zeta potential value of Lys-AuNPs was $+24 \mathrm{mV}$. The charge reversed to a negative value of $-51 \mathrm{mV}$ after the deposition of the negatively charged PSS polyelectrolyte and back to a positive charge of $+18 \mathrm{mV}$ after the deposition of the positively charged PEI polyelectrolyte. The deposition of the layers was also monitored by UV-Vis in the wavelength range $200-800 \mathrm{~nm}$. The Plasmon resonance peak positions ranged from 535 to 545 and were in accordance with the results obtained by DLS measurements. The typical lysozyme absorbance due to the amino acids was also observed at $280 \mathrm{~nm}$. Amide bands of PEI-PSS-LysAuNPs were analyzed by FTIR spectroscopy to evaluate the possibility of thermal denaturation of the lysozyme during the reaction. Amide bands can provide useful information about the secondary structure of proteins such as $\alpha$-helix, $\beta$-sheets, turns or unordered structures. The second derivative spectra of the native lysozyme and Lys-AuNP samples showed both amide I (1700-1600 $\left.\mathrm{cm}^{-1}\right)$ and amide II (1580-1480 $\left.\mathrm{cm}^{-1}\right)$ bands. After FTIR analysis, it was observed that the activity of the protein was not compromised during the reaction processes. In addition, TEM images confirmed the formation of gold nanoparticles using lysozyme as a reducing agent with an average size of $35 \pm 10 \mathrm{~nm}$ ranging between $10-45 \mathrm{~nm}$. 
In the case of the synthesis of AuNPs bioconjugate via MUA-AuNPs, the hydrodynamic diameter of the gold nanoparticles increased during the sequential coating procedures from about 30-40 nm to just below $140 \mathrm{~nm}$ for PEI-PSS-Lys-PSS-PEI-MUA-AuNPs. The deposition of each layer was evaluated by zeta potential values. A reversal of the zeta-potential after each deposition was observed, confirming the negative and positive charge of each compound. The UV-Vis spectra confirmed an increase in the position of the Plasmon resonance peak associated with AuNPs when increasing the size of the nanoparticles in accordance with the results obtained by DLS measurements. Lysozyme absorbance was also observed at $280 \mathrm{~nm}$.

Finally, enzymatic assays were carried out to verify the activity of the enzyme in addition to its observable presence, confirmed by UV-Vis. The residual activities of free lysozyme, encapsulated lysozyme via MUA-AuNPs and bioconjugates based on Lys-AuNPs were monitored for 24 minutes. After 6 minutes the enzymatic activity of the free lysozyme decreased significantly to reach zero activity after 12 minutes due to the degradation of the bacteria walls, which occurred immediately in the presence of native lysozyme. The PEI-PSS-Lys-PSS-PEI-MUAAuNP and PEI-PSS-Lys-AuNP bioconjugates showed an increase during the first 6 minutes; then the PEI-PSS-Lys-PSS-PEI-MUA-AuNPS digested the bacteria walls, decreasing the activity linearly to become zero after 24 minutes; whereas the activity of PEI-PSS-Lys-AuNPs continued to increase after 6 minutes until it reached a plateau. This result shows that the interaction between lysozyme and AuNPs affects the activity of the enzyme retaining it for longer in bioconjugates made via in situ synthesis than those made via citrate reduction prior to stabilization by MUA. In fact, the means of interaction between lysozyme and AuNPs in the asprepared bioconjugates is different: For PEI-PSS-Lys-PSS-PEI-MUA-AuNPS, the interaction of different layers is entirely electrostatic in nature; whereas for Lys-AuNPs, the simultaneous reduction of gold ions into nucleating centers provides a greater probability of synergistic interactions between Lys and NPs. The Au nucleating centers produced upon the reduction of $\mathrm{AuCl}_{4}$ - ions are expected to have a greater potential to break the disulfide groups and form covalent bonds. 



\section{Biomaterials Science}

\section{PAPER}

A) Check for updates

Cite this: Biomater. Sci., 2019, 7, 2511

\section{In situ synthesis, stabilization and activity of protein-modified gold nanoparticles for biological applications}

\author{
Celia Garcia-Hernandez, (10 ${ }^{a, b}$ Allison K. Freese, ${ }^{a}$ Maria L. Rodriguez-Mendez ${ }^{\mathrm{b}}$ and \\ Adam K. Wanekaya*a
}

\begin{abstract}
Herein, we demonstrate the use of lysozyme (Lys) as a model to fabricate a protein carrier system based on gold nanoparticles (AuNPs) via the Layer-by-Layer (LbL) technology. Poly(ethyleneimine) (PEI) and poly (sodium 4-styrenesulfonate) (PSS) were used as cationic and anionic polymers respectively to grow oppositely charged layers. Mild aqueous conditions were utilized to avoid protein denaturation and activity instead of organic solvents that have been used in other encapsulation systems. Two different strategies were used: (A) lysozyme acting as a reducing and stabilizing agent in the formation of AuNPs at a temperature of $45 \pm 2{ }^{\circ} \mathrm{C}$ followed by only two subsequent polymeric layers deposited by $\mathrm{LbL}$, and (B) citrate acting as a reducing agent prior to stabilization of the AuNPs by mercaptoundecanoic acid. Dynamic light scattering. UV-vis spectroscopy. IR spectroscopy and transmission electron microscopy were used to characterize the nanoconjugates. Furthermore, the enzymatic activity of the resulting protein/nanoparticle conjugates was evaluated using the bacteria Micrococcus lysodeikticus as a substrate.
\end{abstract}

Received 25th January 2019 Accepted 27th March 2019 DOI: $10.1039 / \mathrm{c} 9 \mathrm{bm} 00129$ rsc.li/biomaterials-science thesized by using proteins are far more chemically pure and easy to characterize than by using different reducing and stabilizing agents in the presence of proteins. ${ }^{20}$

Herein, we report the in situ synthesis and stabilization of protein-modified gold nanoparticles at a temperature of $45^{\circ} \mathrm{C}$ without the use of activators under mild, near ambient con ditions. The protein acted not only as a reducing agent of the gold salt precursor but also as a stabilizing agent of the resulting gold nanoparticles. The protein/nanoparticle conjugates were coated with poly(ethyleneimine) (PEI), a well-known cationic polymer used for transfection purposes. ${ }^{21}$ We evaluated the enzymatic activity of the resulting $\mathrm{PEI}$-coated protein/nanoparticle conjugates. We also compared the activity of the nanoparticle conjugates with those prepared from the well-known method of synthesizing gold nanoparticles via the reduction of the gold salt precursor by citrate. The citrate-reduced gold nanoparticles were stabilized with mercaptoundecanoic acid (MUA) prior to coating with protein and PEI to form PEI-protein/MUA nanoparticle conjugates.

Proteins have been used to synthesize bioconjugate nanomaterials in the aqueous phase within a temperature range of 40-80 ${ }^{\circ} \mathrm{C}$. Lead sulphide ( $\mathrm{PbS}$ ) nanocrystals have been synthesized using bovine serum albumen (BSA), an important blood protein, as the capping/stabilizing agent in the range of $40-80^{\circ} \mathrm{C} .{ }^{22}$ The same protein was utilized to develop BSA-conjugated AuNPs to explore their applications as drug delivery vehicles. The BSA-conjugated AuNPs were fabricated by react- 
Paper IX: In situ synthesis, stabilization and activity of protein-modified gold nanoparticles for biological applications.

View Artide Online

Paper

Biomaterials Science

ing $\mathrm{BSA}$ with $\mathrm{HAuCl}_{4}$ at a temperature of $70{ }^{\circ} \mathrm{C}$ for $6 \mathrm{~h}$ under static conditions. ${ }^{23}$ Cytochrome $\mathrm{c}$ protein has also been used to synthesize gold nanoparticles under mild conditions in a temperature range of $40-80^{\circ} \mathrm{C} .^{20}$

Lysozyme was selected as the model protein for various reasons. Lysozyme is a well-characterized protein with a high isoelectric point ( $\mathrm{pI}$ 11.35). ${ }^{24}$ In addition, lysozyme is stable over wide temperature and $\mathrm{pH}$ ranges, making it suitable for a variety of synthetic approaches. These properties also make this protein attractive for use as a model protein for various applications. Therefore, a large number of attempts have emerged to investigate different ways for lysozyme immobiliz ation, or encapsulation, many of which include Layer-by-Laye (LbL) steps during the process. ${ }^{25-27}$ Therefore, LbL structures have been constructed to immobilize proteins such as catalase, $\alpha$-chymotrypsin, cholesterol oxidase, glucose isomerase, $\beta$-glucosidase, urease, peroxidase and glucose oxidase. ${ }^{28-35}$

\section{Experimental}

\subsection{Materials}

All chemicals and solvents were of reagent grade and used without further purification. Chloroauric acid $\left(\mathrm{HAuCl}_{4} \cdot 3 \mathrm{H}_{2} \mathrm{O}\right)$ poly(ethyleneimine) (branched, $25 \mathrm{kDa}$ ) (PEI), poly(sodium 4-styrenesulfonate) (70 kDa) (PSS), 11-mercapto-undecanoic acid (MUA), lysozyme (Lys) from chicken egg white, the bac teria Micrococcus lysodeikticus and Corning syringe filte membrane (with diameter $15 \mathrm{~mm}$ and pore size $0.2 \mu \mathrm{m}$ ) were purchased from Sigma-Aldrich (St Louis, MO, USA). Sodium citrate dihydrate and sodium phosphate monobasic were purchased from Spectrum Chemical Manufacturing Group (Gardena, CA, USA). Sodium phosphate dibasic was purchased from Mallinckrodt Chemical Works (St Louis, MO, USA) Deionized water with a resistivity of $18.2 \mathrm{M} \Omega \mathrm{cm}$ was used to prepare all the solutions.

\subsection{Instrumentation and characterization}

Purification of colloidal gold nanoparticle suspensions was performed using a micro-centrifuge D3024 from Scilogex (Rocky Hill, CT, USA). Dynamic light scattering (DLS) and zetapotential measurements were carried out using a NanoBrook Omni from Brookhaven Instruments (Holtsville, NY, USA). A Cary $60 \mathrm{UV}$-vis spectrophotometer from Agilent Technologies (Santa Clara, CA, USA) was used for recording UV-vis spectra and for the enzymatic activity assays. Absorbance spectra were recorded from 200 to $800 \mathrm{~nm}$. For particle size measurements using DLS, the samples were re-suspended in $1 \mathrm{mM} \mathrm{NaCl}$ and filtered with $0.2 \mu \mathrm{m}$ filters. Infrared spectra were recorded on a Jasco Model FT/IR-6600 Spectrometer (Jasco, Tokyo, Japan) with a diamond ATR crystal accessory. The software used for FTIR data collection was Spectra Manager II (Jasco, Tokyo, Japan). Before the analysis the instrument was purged with nitrogen for $10 \mathrm{~min}$. As a reference, the background spectrum of air (100 BKG) was recorded before the acquisition of the sample spectrum. Spectra were recorded at $25^{\circ} \mathrm{C}$ with a resolu- tion of $2 \mathrm{~cm}^{-1}$ and 300 scans were averaged for each spectrum (scan 1800-1300 $\mathrm{cm}^{-1}$ ). Transmission electron microscopy (TEM) analysis of Lys-AuNPs was carried out using a TEM JEOL JEM-1011 HR (JEOL Ltd, Tokyo, Japan) operating at $100 \mathrm{kV}$. ImageJ software was used to calculate particle size distribution from transmission electron micrographs.

\subsection{Synthesis of lysozyme-gold nanoparticle conjugates} (Scheme 1Aa)

Gold nanoparticles were synthesized using lysozyme as a reducing agent. The synthesis of Lys-AuNPs was done via modification of a previous protocol. ${ }^{20}$ Briefly, $25 \mathrm{~mL}$ of $0.25 \mathrm{mM}$ $\mathrm{HAuCl}_{4}$ and $250 \mu \mathrm{L}$ of $700 \mu \mathrm{M}$ lysozyme were mixed in an Erlenmeyer flask at room temperature (final concentration ratio $\mathrm{HAuCl}_{4} / \mathrm{Lys}=36$ ). After the components were mixed, the reaction mixture was kept in a water bath at $45 \pm 2{ }^{\circ} \mathrm{C}$ for 6 hours under static conditions. The reaction mixture was then cooled down to room temperature and kept overnight under ambient conditions. The Lys-AuNPs were purified by centrifugation at $2380 \mathrm{~g}$ for 5 minutes followed by washing in deionized water. The Lys-AuNPs were re-suspended in $1 \mathrm{mM} \mathrm{NaCl}$ for subsequent deposition with layers of poly(sodium 4-styrenesulfonate) (PSS) and poly(ethyleneimine) (PEI).

\subsection{Coating of Lys-AuNPs (Scheme 1Ab)}

The Layer-by-Layer technique was used for further deposition following the coating protocols previously described in the literature $^{36,37}$ with some modifications. A colloidal solution of Lys-AuNPs in $1 \mathrm{mM} \mathrm{NaCl}$ was added dropwise to a stirred solution of PSS under ambient conditions. The concentration of PSS in the final reaction solution was $5 \mathrm{mg} \mathrm{mL}^{-1}$. Stirring was continued over $1 \mathrm{~h}$. The resulting PSS-Lys-AuNPs were purified by centrifugation as before $(2380 \mathrm{~g}$ for $5 \mathrm{~min}$ at room temperature followed by washing with deionized water), re-suspended in $1 \mathrm{mM} \mathrm{NaCl}$ and added dropwise to a stirred solution of PEI under ambient conditions. The concentration of PEI in the final reaction solution was $5 \mathrm{mg} \mathrm{mL}^{-1}$. Stirring was continued over $1 \mathrm{~h}$. The resulting PEI-PSS-Lys-AuNPs were purified by centrifugation and re-suspended in $1 \mathrm{mM} \mathrm{NaCl}$ as before.

\subsection{Synthesis of gold nanoparticles (Scheme $1 \mathrm{Bb}$ )}

A colloidal solution of citrate-stabilized AuNPs was synthesized according to the method introduced by Frens ${ }^{11}$ based on the Turkevich method ${ }^{10}$ using sodium citrate as a reducing and stabilizing agent. Briefly, $500 \mathrm{~mL}$ of $0.01 \% \mathrm{w} / \mathrm{v} \mathrm{HAuCl}_{4}$ solution was heated to $100{ }^{\circ} \mathrm{C}$ and then $3.6 \mathrm{~mL}$ of $1 \% \mathrm{w} / \mathrm{v}$ sodium citrate solution was added dropwise with stirring. The reaction mixture was stirred and heated for 20 minutes. The color of the solution turned from light yellow to dark red. AuNP size can be adjusted by varying the $\mathrm{HAuCl}_{4} /$ citrate ratio. The AuNPS obtained from this synthesis resulted in a size of about 30-40 $\mathrm{nm}$ and were stabilized by a cover of citrate ions preventing the aggregation of the particles (Scheme 1Ba). The AuNPs were further stabilized using 11-mercapto-undecanoic acid (MUA) as a dispersant. Briefly, the $\mathrm{pH}$ of the AuNP suspension 
$\begin{array}{lr}\text { View Article Online } \\ \text { Biomaterials Science } & \text { Paper }\end{array}$

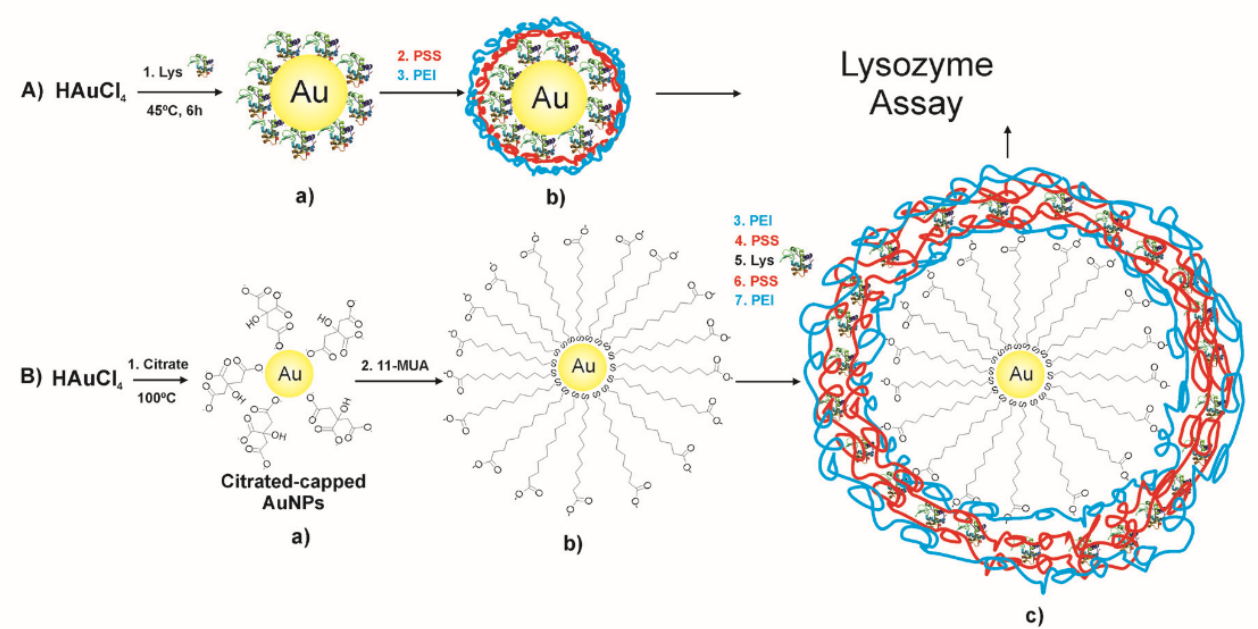

Scheme 1 Overview of the experimental schematic showing the synthesis of Lys-AuNPs (Aa), PEI-PSS-Lys-AuNPs (Ab), citrate-AuNPs (Ba), MUA-AuNPs (Bb) and PEI-PSS-Lys-PSS-PEI-MUA-AuNPs (Bc). (Not to scale).

was adjusted to 11.00 with $\mathrm{NaOH}$. After this, MUA (20 $\mathrm{mg} \mathrm{mL} \mathrm{m}^{-1}$ in $10 \mathrm{mM} \mathrm{NaOH}$ ) was added dropwise to the AuNP suspension under stirring to give a final concentration of $0.1 \mathrm{mg} \mathrm{mL} \mathrm{m}^{-1}$ of MUA. Stirring was continued under dark conditions over 3 days to ensure a stable surface modification. After 3 days, MUA-AuNPs were purified by centrifugation to remove the excess MUA. Centrifugation conditions depend strongly on the particle size. Purification was done by centrifuging the MUA-AuNPs at $2380 \mathrm{~g}$ for $5 \mathrm{~min}$ at room temperature followed by washing with deionized water. The nanoparticles were re-suspended in $1 \mathrm{mM} \mathrm{NaCl}$. Unpurified MUA-AuNP nanoparticles can be stored over weeks. They were tested every single week to verify the size.

\subsection{Coating of MUA-AuNPs (Scheme 1Bc)}

The coating of MUA-AuNPs was done using the LbL technique as was previously described by sequential layering with poly (ethyleneimine) (PEI) and poly(sodium 4-styrenesulfonate) (PSS) respectively. MUA-AuNPs were resuspended in $1 \mathrm{~mm}$ $\mathrm{NaCl}$ and were added dropwise to a solution of PEI under stirring at room temperature. The final concentration of the PEI solution was $5 \mathrm{mg} \mathrm{mL}{ }^{-1}$. Stirring was continued for $1 \mathrm{~h}$. Afterwards, PEI-MUA-AuNPs were purified as before $(2380 \mathrm{~g}$ for $5 \mathrm{~min}$ at room temperature followed by washing with deionized water). After purification the nanoparticles were resuspended in $1 \mathrm{mM} \mathrm{NaCl}$ and the PSS layer was deposited by LbL. PEI-MUA-AuNPs were added dropwise to a PSS solution under stirring at room temperature up to a final PSS concentration of $5 \mathrm{mg} \mathrm{mL}^{-1}$. After $1 \mathrm{~h}$ the PSS-PEI-MUA-AuNP suspension was purified as before and resuspended in $1 \mathrm{mM} \mathrm{NaCl}$. The resulting PSS-PEI-MUA-AUNPs were coated with lysozyme as follows: $10 \mathrm{mg} \mathrm{mL}{ }^{-1}$ lysozyme in phosphate buffer solution ( $\mathrm{pH} 7.00)$ was added dropwise to a stirred colloidal solution of
PSS-PEI-MUA-AuNPS under ambient conditions. The final concentration of lysozyme in the reaction mixture was $120 \mu \mathrm{M}$. Stirring was continued over $3 \mathrm{~h}$. The resulting Lys-PSS-PEIMUA-AuNPs were purified as described before. They were then sequentially coated with layers of PSS and PEI as described previously forming PEI-PSS-Lys-PSS-PEI-MUA-AUNPs (Scheme 1Bc).

\subsection{Lysozyme activity assay}

The bacteria Micrococcus lysodeikticus was used as a substrate for the enzymatic action of lysozyme. In order to compare the protein gold nanoparticles with free lysozyme, an estimated concentration of encapsulated lysozyme was determined spectrophotometrically using an extinction coefficient of 2.63 $\mathrm{mL} \mathrm{mg} \mathrm{m}^{-1} \mathrm{~cm}^{-1}$ when measuring the absorbance at $280 \mathrm{~nm}$ after lysozyme deposition. ${ }^{38,39}$ Micrococcus lysodeikticus solution was prepared by dissolving $50 \mathrm{mg}$ of bacteria in $100 \mathrm{~mL}$ of $50 \mathrm{mM}$ buffer phosphate ( $\mathrm{pH}$ 6.25). The AuNP samples and free lysozyme were dissolved in $50 \mathrm{mM}$ buffer phosphate (pH 6.25). $600 \mu \mathrm{L}$ of the sample was added to $3 \mathrm{~mL}$ of bacteria solution at room temperature and mixed. The decrease in the absorbance was monitored at $600 \mathrm{~nm}$ for 30 minutes at room temperature. The enzymatic activities were determined from the slope of the fitted line when representing the absorbance decay plots and using eqn (1).

$$
\frac{\text { Units }}{\mathrm{mL}_{\text {enzyme sample }}}=\frac{\left(\Delta \mathrm{Abs}_{600} / \mathrm{min} \text { test }-\Delta \mathrm{Abs}_{600} / \mathrm{min} \text { blank }\right)}{0.001 \cdot(0.6)}
$$

where 0.001 is the change in the absorbance per minute for lysozyme and 0.6 is the added volume of the sample to the bac 
teria solution during the tests in $\mathrm{mL}$. The blank used for the assays was $50 \mathrm{mM}$ phosphate buffer.

\section{Results and discussion}

\subsection{Synthesis and coating of Lys-AuNPs}

Fig. 1 shows the visible absorption spectra of the synthesis of Lys-AuNPs from the beginning to the end of the reaction process. Lysozyme acted as the reducing and capping agent of the AuNPs. There was a noticeable and progressive increase in the Au plasmon resonance peak after $4 \mathrm{~h}$. This peak reached its maximum at approximately $12 \mathrm{~h}$. The Lys-AuNPs were purified by sequential centrifugation and rinsing.

Fig. $2 \mathrm{~A}$ and Table 1 shows DLS spectroscopy results for the hydrodynamic diameter and polydispersity indexes of Lys-AuNPs before and after PSS and PEI deposition to form PSS-Lys-AuNPs and PEI-PSS-Lys-AuNPs, respectively. The hydrodynamic diameters of Lys-AuNPs and PSS-Lys-AuNPs were about $60 \mathrm{~nm}$. The diameter increased to $90 \mathrm{~nm}$ after PEI deposition. In most cases, the polydispersity indexes were approximately $30 \%$ or lower.

In addition to the hydrodynamic diameter, the deposition of each layer was monitored by measuring the zeta potential values of the nanoconjugates (Fig. 2B and Table 1). Zeta potentials not only provide quantitative values of the overall surface charges of the nanoparticles, but also predict colloidal stability. A large value of the zeta potential, regardless of the charge, is a prediction of colloidal stability due to extra electrostatic repulsion between similarly charged particles. Therefore, zeta potentials also confirm the successful deposition of polyelectrolytes on the particle surface.

Initially before the coating the zeta potential value of LysAuNPs was $+24 \mathrm{mV}$. This is expected due to the overall positive charge of lysozyme. The charge reversed to a negative value of

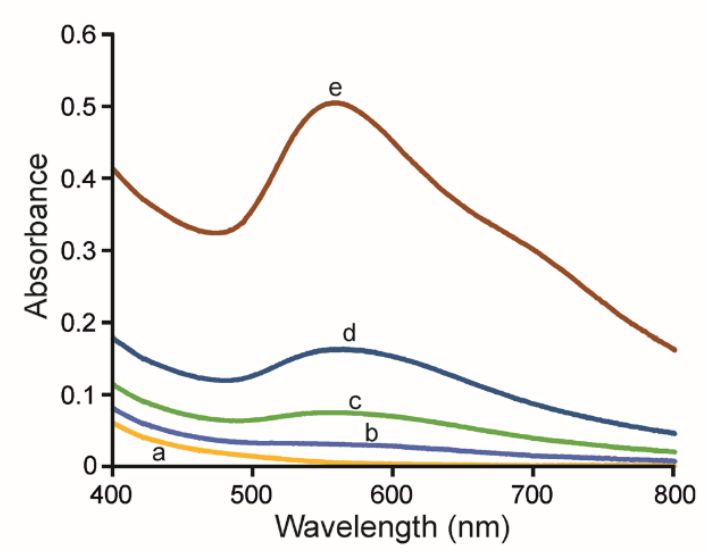

Fig. 1 Time-dependent absorbance spectra of AuNPs by reducing $\mathrm{HAuCl}_{4}$ with lysozyme at $45 \pm 2{ }^{\circ} \mathrm{C}$. (a) Immediately after the start of the reaction and after (b) $4 \mathrm{~h}$, (c) $5 \mathrm{~h}$, (d) $6 \mathrm{~h}$ and (e) $12 \mathrm{~h}$.
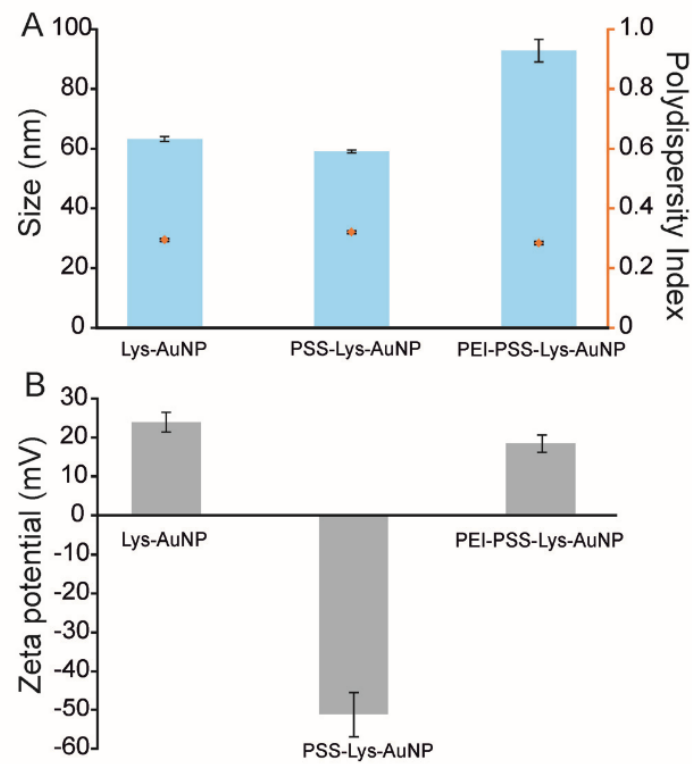

Fig. 2 (A) Hydrodynamic diameter and polydispersity index of PEI PSS-Lys-AuNPs. (B) Zeta-potential values before and after sequential coating steps of the Lys-AuNPs.

Table 1 Hydrodynamic diameter, polydispersity index and zeta poten tial values of Lys-AuNPs and Lys-AuNPs coated with PSS and PE

\begin{tabular}{llll}
\hline & \multicolumn{2}{l}{ DLS measurements } & \\
\cline { 2 - 3 } & Size $(\mathrm{nm})$ & Polydispersity & ZP $(\mathrm{mV})$ \\
\hline Lys-AuNPs & $63.24 \pm 0.86$ & $0.294 \pm 0.004$ & $23.96 \pm 2.56$ \\
PSS-LyS-AuNPs & $59.07 \pm 0.46$ & $0.320 \pm 0.004$ & $-51.16 \pm 5.77$ \\
PEI-PSS-Lys-AuNPs & $92.79 \pm 3.81$ & $0.284 \pm 0.005$ & $18.47 \pm 2.25$
\end{tabular}

$-51 \mathrm{mV}$ after deposition of the negatively charged PSS polyelectrolyte and back to a positive charge of $+18 \mathrm{mV}$ after deposition of the positively charged PEI polyelectrolyte.

The deposition of the layers was monitored by UV-vis in the wavelength range of 200 to $800 \mathrm{~nm}$ (Fig. 3). The Au plasmon resonance peaks were in the 535 to $545 \mathrm{~nm}$ range. The shifts observed in the plasmon resonance peak position showed a similar trend to that of the results obtained by DLS measurements. The typical lysozyme absorbance due to the amino acids was also observed at $280 \mathrm{~nm}$. The characteristic absorbance peak of PSS at $260 \mathrm{~nm}$ was present after the deposition of the polymer.

In order to evaluate the possibility of thermal denaturation of lysozyme during the reaction with $\mathrm{HAuCl}_{4}$ at $45 \pm 2{ }^{\circ} \mathrm{C}$, FTIR spectroscopy was used to monitor the secondary structural nature of lysozyme. Lysozyme has been one of the most common protein models used in thermal denaturation assays. $^{40-42}$ Amide bands can provide useful information 


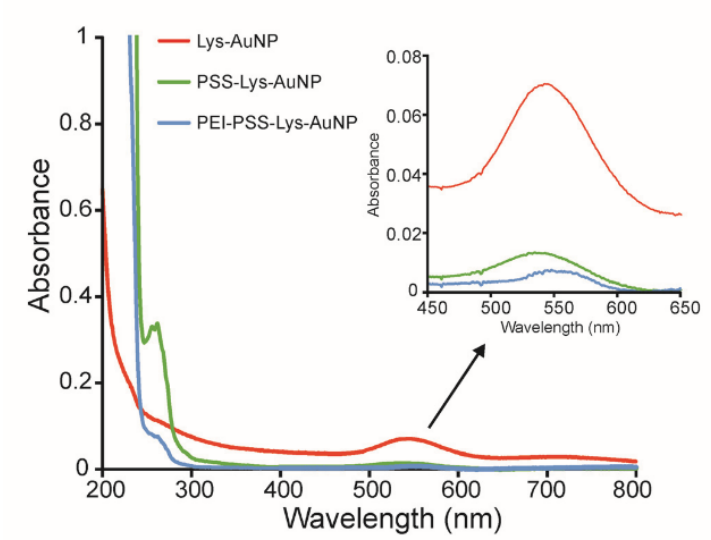

Fig. 3 Absorbance spectra of PEI-PSS-Lys-AuNPs at different layers. Inset: Plasmon peak position shift.

about the secondary structure of proteins such as $\alpha$-helix, $\beta$-sheets, turns or unordered structures. Amides I and II are the major bands in the protein IR spectrum. Amide I $\left(1700-1600 \mathrm{~cm}^{-1}\right)$ is the most intense absorption band in proteins and is due to $\mathrm{C}=\mathrm{O}$ stretching vibrations of the peptide bonds. The exact band position is determined by the backbone conformation and the hydrogen bonding pattern. Amide II (1580-1480 $\mathrm{cm}^{-1}$ ) derives mainly from in-plane $\mathrm{N}-\mathrm{H}$ bending and the rest of the potential energy arises from the $\mathrm{C}-\mathrm{N}$ and $\mathrm{C}-\mathrm{C}$ stretching vibrations. Amide $\mathrm{III}$ and $\mathrm{V}$ are very complex bands dependent on the details of the force field, the nature of side chains and hydrogen bonding. Due to the technical and theoretical limitations, only amide I and amide III bands are used for investigating the secondary protein structure. ${ }^{43-46}$ Amide I modes, however, are the most extensively used in such studies. ${ }^{43-45}$

The relationship between the amide I band position and the type of secondary structure is best recognized by analyzing the IR spectra of simple homopolypeptides that fold into welldefined structures (purely alpha-helical or beta-sheet). The amide I band consists of many overlapping component bands that represent different structural elements (e.g. alpha helices, beta sheets, turns, and non-ordered or irregular structures). Extraction of single subcomponent bands can be achieved using curve-fitting approaches to assign each subcomponent to a particular protein secondary structure (e.g. Gaussian curve-fitting). ${ }^{43}$

The second derivative spectra of native lysozyme and LysAuNP samples showed both amide I $\left(1700-1600 \mathrm{~cm}^{-1}\right)$ and amide II (1580-1480 $\mathrm{cm}^{-1}$ ) bands (Fig. 4). Moreover, Gaussian curve-fitting of the amide I band showed typical components already reported for lysozyme (Fig. 5). ${ }^{40,41,45,47}$ For Lys-AuNPs, the assignment of bands were $\beta$-sheet secondary structure at ca. $1628 \mathrm{~cm}^{-1}$ and $1681 \mathrm{~cm}^{-1}$; $\alpha$-helical structure at $1652 \mathrm{~cm}^{-1}$; $\beta$-turns at $c a .1667 \mathrm{~cm}^{-1}$; and random coil structures at $c a$. $1638 \mathrm{~cm}^{-1}$ (Table 2). It has been reported that thermal dena-

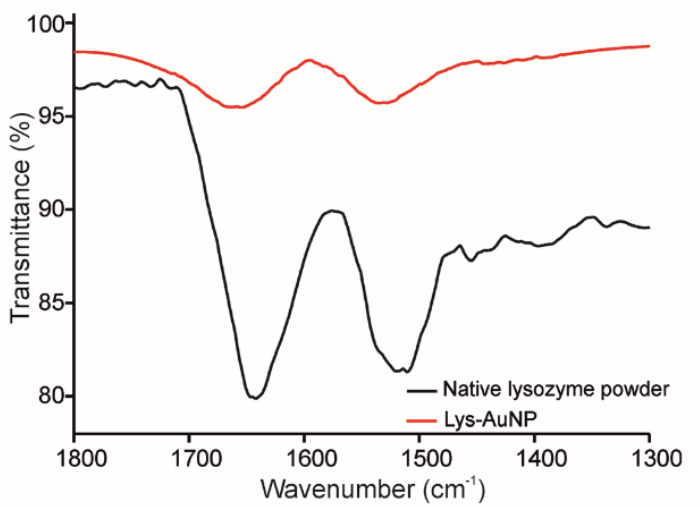

Fig. 4 ATR-FTIR spectra of lysozyme stabilized AuNPs and native lysozyme.

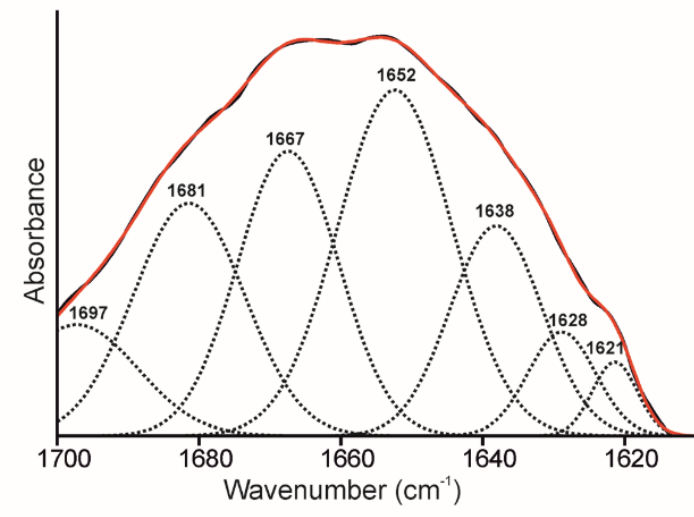

Fig. 5 Gaussian curve-fitting of amide I FTIR spectra of lysozyme stabilized AuNPs (black curve represents the original spectrum and red curve represents the overall fit).

Table 2 Secondary structure assignment of lysozyme in the amide I region

\begin{tabular}{ll}
\hline Frequency $\left(\mathrm{cm}^{-1}\right)$ & Assignment \\
\hline 1628,1681 & $\beta$-Sheet \\
1638 & Random coil \\
1652 & $\alpha$-Helix \\
1667 & Turns
\end{tabular}

turation causes an increase in $\beta$ content in amide I accompanied by a decrease in the $\alpha$-helix content. ${ }^{40}$ Such "helix to sheet" transitions have been observed in the irreversible thermal denaturation of many proteins. ${ }^{40}$ Therefore, it reasonable to conclude that the integrity of the protein was not compromised during the reaction processes. 
Paper

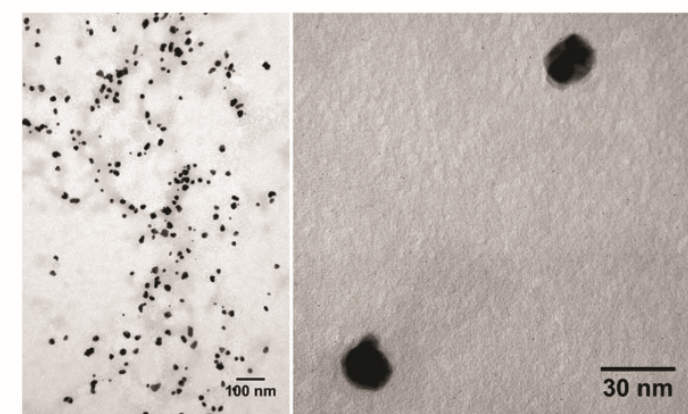

Fig. 6 TEM images of the synthesized Lys-AuNPs.

Moreover, TEM images also confirmed the formation of gold nanoparticles using lysozyme as a reducing agent. Lysozyme-gold nanoparticles were generally uniformly dispersed and mainly spherical in morphology (Fig. 6). The average size of Lys-AuNPs was $35 \pm 10 \mathrm{~nm}$ ranging between 10-45 nm. Particle size results from TEM images were smaller than the ones obtained from DLS analysis $(\sim 60 \mathrm{~nm})$. The differences in particle size from DLS analysis and TEM imaging is not unusual. In general, particle sizes from DLS spectra are larger than those obtained from TEM images. DLS measures the hydrodynamic diameter of particles in liquids while TEM results are from the direct imaging of dry particles.

\subsection{Synthesis and coating of MUA-AuNPs}

Fig. 7A and Table 3 show the hydrodynamic diameter of the gold nanoparticles, including the polydispersity index as measured by DLS spectroscopy for PEI-PSS-Lys-PSS-PEIMUA-AuNPs. The size of AuNPs increased during the sequential coating procedures from about $30-40 \mathrm{~nm}$ to just below $140 \mathrm{~nm}$ for PEI-PSS-Lys-PSS-PEI-MUA-AuNPs. We have found that different molecular weights of PEI and PSS did not appear to influence the final particle size. However, the molecular weight and the degree of branching of PEI has been reported to influence the efficacy of PEI based vectors in delivering plasmid DNA and siRNAs. ${ }^{48}$ The most suitable PEI molecular weight ranges between 5 and $25 \mathrm{kDa}$. Higher molecular weights are associated with an increase in the cytotoxicity due to aggregation of huge clusters of the cationic polymer on the outer cell membrane and therefore inducing necrosis. ${ }^{48}$

The deposition of each layer was confirmed by zeta potential values (Fig. 7B and Table 3). A reversal of the zeta-potential after each deposition was observed.

Unpurified MUA-AuNPs were stored at room temperature for weeks. The hydrodynamic diameter was periodically tested to confirm that no aggregation had occurred (Fig. 8). The size of MUA-AuNPs ranged between 39-45 $\mathrm{nm}$ over weeks.

The deposition of the layers was also monitored by UV-vis in the wavelength range of 200 to $800 \mathrm{~nm}$ (Fig. 9). There was a gradual shift of the Au plasmon resonance peak from $525 \mathrm{~nm}$ to $565 \mathrm{~nm}$ as the layers were deposited on the particles.
View Article Online

Biomaterials Science
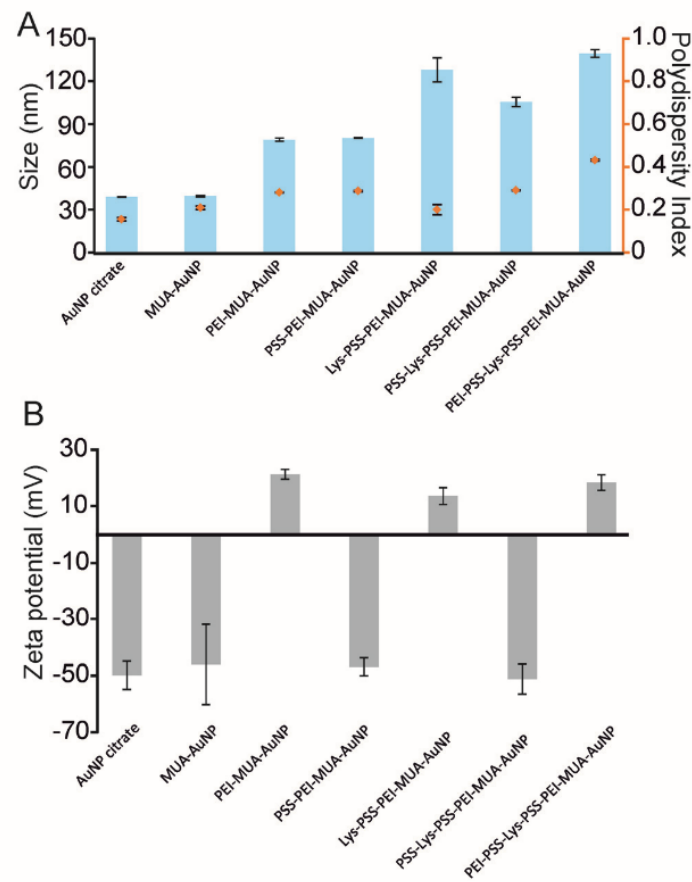

Fig. 7 (A) Hydrodynamic diameter and polydispersity index of PEIPSS-Lys-PSS-PEI-MUA-AuNPs. (B) The effect of the zeta-potential of the subsequent coating steps.

Lysozyme exhibited the typical protein absorbance at $280 \mathrm{~nm}$. The characteristic absorbance peak of PSS at $260 \mathrm{~nm}$ was also observed after the deposition of the polymer.

\subsection{Lysozyme activity assay}

Finally, enzymatic assays were carried out to verify the activity of the enzyme in addition to its observable presence confirmed by UV-vis. Using an extinction coefficient of $2.63 \mathrm{~mL}$ $\mathrm{mg}^{-1} \mathrm{~cm}^{-1}$, the lysozyme concentration was estimated to be $3 \mu \mathrm{M}$ for PEI-PSS-Lys-PSS-PEI-MUA-AuNPs and $2.7 \mu \mathrm{M}$ for PEI-PSS-Lys-AuNPs. The free lysozyme concentration used for the enzymatic assay was $3 \mu \mathrm{M}$ in order to compare enzymatic activities. The enzymatic activity of free lysozyme, encapsulated lysozyme via MUA-AuNPs and bioconjugates based on LysAuNPs was monitored for 24 minutes. Fig. 10 shows the enzymatic activities of free lysozyme and the lysozyme-gold nanoparticles. Within 6 minutes the enzymatic activity of free lysozyme decreased significantly and it became almost zero in 12 minutes. Bacteria wall degradation occurred immediately in the presence of pure native lysozyme such that within 12 minutes the lysozyme had digested all the bacteria used in the assay.

On the other hand, PEI-PSS-Lys-PSS-PEI-MUA-AuNPs and PEI-PSS-Lys-AuNP bioconjugates exhibited a different behaviour. The enzymatic activity increased in the first 6 minutes 
Table 3 Hydrodynamic diameter, polydispersity index and zeta potential values of PEI-PSS-Lys-PSS-PEI-MUA-AuNPs at different layers

\begin{tabular}{|c|c|c|c|}
\hline & \multicolumn{2}{|c|}{ DLS measurements } & \multirow{2}{*}{$\begin{array}{l}\text { ELS measurements } \\
\text { ZP (mV) }\end{array}$} \\
\hline & Size $(\mathrm{nm})$ & Polydispersity & \\
\hline AuNP citrate & $38.82 \pm 0.19$ & $0.154 \pm 0.008$ & $-49.90 \pm 4.96$ \\
\hline MUA-AuNP & $39.44 \pm 0.48$ & $0.208 \pm 0.008$ & $-46.10 \pm 14.16$ \\
\hline PEI-MUA-AUNP & $79.18 \pm 1.12$ & $0.280 \pm 0.002$ & $21.25 \pm 1.79$ \\
\hline PSS-PEI-MUA-AuNP & $80.38 \pm 0.28$ & $0.287 \pm 0.003$ & $-46.90 \pm 3.16$ \\
\hline Lys-PSS-PEI-MUA-AuNP & $127.95 \pm 8.50$ & $0.199 \pm 0.023$ & $13.57 \pm 2.93$ \\
\hline PSS-LyS-PSS-PEI-MUA-AuNP & $105.72 \pm 3.33$ & $0.290 \pm 0.003$ & $-51.24 \pm 5.39$ \\
\hline PEI-PSS-LyS-PSS-PEI-MUA-AuNP & $139.51 \pm 2.70$ & $0.431 \pm 0.004$ & $18.36 \pm 2.77$ \\
\hline
\end{tabular}

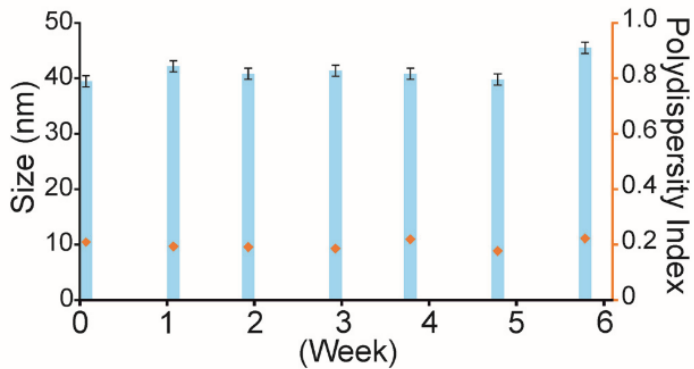

Fig. 8 Hydrodynamic diameter of MUA-AuNPs up to 6 weeks.

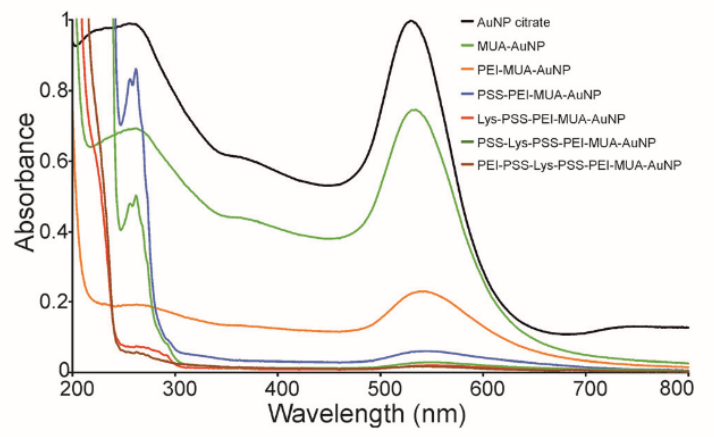

Fig. 9 Absorbance spectra of PEI-PSS-Lys-PSS-PEI-MUA-AuNPs at different layers.

due to protection from the polymeric layers. After that time PEI-PSS-Lys-PSS-PEI-MUA-AuNPs exhibited a decrease in activity to zero in 24 minutes. Surprisingly, the activity of PEIPSS-Lys-AuNPs continued increasing after 6 minutes to reach a plateau. This implies that the interaction between lysozyme and AuNPs occurs in a different manner that modifies the activity of the enzyme.

It is therefore evident that the reaction conditions (high temperature for a long time) in the formation of Lys-AuNPs did not appear to affect the activity of the protein.

Moreover, the in situ synthesis and stabilization of LysAuNPs by lysozyme has considerable advantages compared to

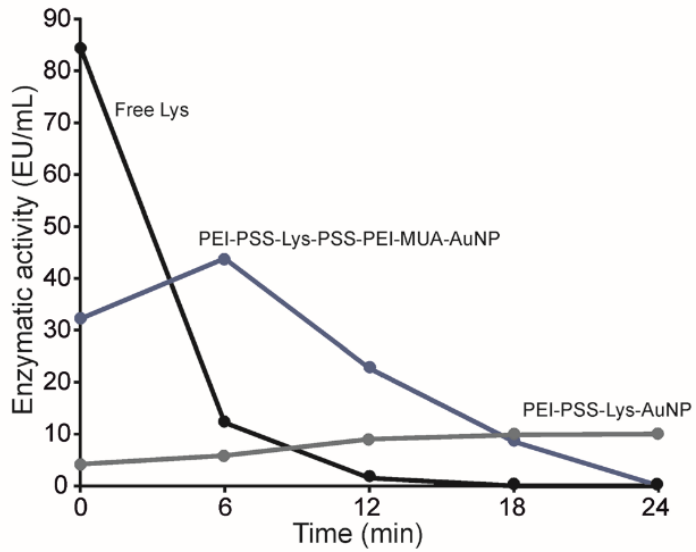

Fig. 10 Enzymatic activities of free lysozyme and the lysozyme-gold nanoparticles expressed as EU per $\mathrm{mL}_{\text {enzyme sample. }}$

the AuNPs fabricated via citrate reduction prior to stabilization by MUA. The former procedure is much shorter and involves less deposition. Moreover, the simultaneous reduction of gold ions into nucleating centers provides synergistic interactions between Lys and the nanoparticles. The Au nucleating centers produced upon the reduction of $\mathrm{AuCl}_{4}{ }^{-}$ions are expected to have greater potential to break the disulfide groups and form covalent bonds. ${ }^{20}$ On the other hand, the interaction of different layers in PEI-PSS-Lys-PSS-PEI-MUA-AuNPS is entirely electrostatic in nature. Electrostatic interactions are much weaker, thus exposing the enzyme to conditions that may impact its activity.

\section{Conclusions}

In summary, we have demonstrated the in situ synthesis and stabilization of protein modified gold nanoparticles without the use of activators under mild and near ambient conditions. The protein/nanoparticle conjugates were coated with poly (ethyleneimine). The enzymatic activity of the resulting protein/nanoparticle conjugates was evaluated. The enzymatic activity was compared with those prepared by the well-known 
Paper IX: In situ synthesis, stabilization and activity of protein-modified gold nanoparticles for biological applications.

View Artide Online

Paper

Biomaterials Science

citrate reduction of chloroauric acid followed by the layer-by layer coating procedure. The activity of the protein was much longer for the protein/nanoparticle conjugates than for the native protein. Also, the protein/nanoparticle conjugates synthesized from protein reduction of chloroauric acid seem to retain the activity for a much longer time than those synthesized from citrate reduction. Further standardized experiments will be conducted to confirm this. While the utility of the technique was demonstrated for stabilization and activity of lysozyme, it could easily be adapted and optimized for other proteins. However, as the isoelectric point (pI) of most proteins is in the pH range of 4 to 7 it is very important to consider the $\mathrm{pH}$ of the solution in which the LbL-based layers will be performed as well as the charge of the polymeric materials used to encapsulate the protein. Other proteins, with a pI greater than 7 , to be used as positive layer following the protocol described in this work include histone, cytochrome c, ribonuclease A, hemoglobin and myoglobin among others. It, therefore, has great potential in in vivo cytosolic protein delivery.

\section{Conflicts of interest}

There are no conflicts to declare.

\section{Acknowledgements}

Celia Garcia-Hernandez wishes to acknowledge A. K. Wanekaya and Missouri State University, grant BOCYL-D-24112015-9 from the Junta de Castilla $\mathrm{y}$ León and funding from the University of Valladolid (EB-17).

\section{References}

1 S. Barber, M. Abdelhakiem, K. Ghosh, L. Mitchell, R. Spidle, B. Jacobs, L. Washington, J. Li, A. Wanekaya G. Glaspell and R. K. Delong, J. Nanosci. Nanotechnol., 2011, 11, 10309-10319, DOI: 10.1166/jnn.2011.5013.

2 H. Gann, G. Glaspell, R. Garrad, A. Wanekaya, K. Ghosh, L. Cillessen, A. Scholz, B. Parker, M. Warner and R. K. Delong, J. Biomed. Nanotechnol., 2010, 6, 37-42, DOI: 10.1166/jbn.2010.1100.

3 D. A. Shah, S.-J. Kwon, S. S. Bale, A. Banerjee, J. S. Dordick and R. S. Kane, Biomaterials, 2011, 32, 3210-3219, DOI: 10.1016/j.biomaterials.2010.11.077.

4 J. M. Hobbs, N. N. Patel, D. W. Kim, J. K. Rugutt and A. K. Wanekaya, J. Chem. Educ., 2013, 90, 1222-1226, DOI: 10.1021/ed300429a.

5 M. B. Wayu, R. T. Spidle, T. Devkota, A. K. Deb, R. K. Delong, K. C. Ghosh, A. K. Wanekaya and C. C. Chusuei, Electrochim. Acta, 2013, 97, 99-104, DOI: 10.1016/j.electacta.2013.02.028.

6 S. Foster, C. L. Duvall, E. F. Crownover, A. S. Hoffman and P. S. Stayton, Bioconjugate Chem., 2010, 21, 2205-2212, DOI: $10.1021 / \mathrm{bc} 100204 \mathrm{~m}$.
7 J. B. Xie, Y. Li, Y. Cao, C. Xu, M. Xia, M. Qin, J. W. Wei and W. Wang, Biomater. Sci., 2013, 1, 1216-1222, DOI: 10.1039/ c3bm60174a.

8 L. Lamboni, M. Gauthier, G. Yang and Q. Wang, Biotechnol. Adv. $\quad 2015, \quad 33, \quad 1855-1867$, DOI: $10.1016 / \mathrm{j}$. biotechadv.2015.10.014.

9 J. Y. Zong, S. L. Cobb and N. R. Cameron, Biomater. Sci, 2017, 5, 872-886, DOI: $10.1039 / \mathrm{c} 7$ bm00006e.

10 J. Turkevich, P. C. Stevenson and J. Hillier, Discuss. Faraday Soc., 1951, 11, 55-75, DOI: 10.1039/DF9511100055.

11 G. Frens, Nat. Phys. Sci. 1973, 241, 20, DOI: 10.1038/ physci241020a0.

12 H. Huang and X. Yang, Biomacromolecules, 2004, 5, 23402346, DOI: $10.1021 / \mathrm{bm} 0497116$.

13 A. Singh, S. Hede and M. Sastry, Small, 2007, 3, 466-473, DOI: $10.1002 / \mathrm{smll} .200600413$.

14 H. Zhu, M. Du, M. Zou, C. Xu, N. Li and Y. Fu, J. Mater. Chem., 2012, 22, 9301-9307, DOI: 10.1039/C2JM16569D.

15 J. Xie, Y. Zheng and J. Y. Ying, J. Am. Chem. Soc., 2009, 131, 888-889, DOI: $10.1021 /$ ja806804u.

16 H. Kawasaki, K. Hamaguchi, I. Osaka and R. Arakawa, $A d v$. Funct. Mater., 2011, 21, 3508-3515, DOI: 10.1002/ adfm.201100886.

17 T.-H. Chen and W.-L. Tseng, Small, 2012, 8, 1912-1919, DOI: $10.1002 / \mathrm{smll} .201102741$.

18 Y. Leng, L. Fu, L. Ye, B. Li, X. Xu, X. Xing, J. He, Y. Song, C. Leng, Y. Guo, X. Ji and Z. Lu, Sci. Rep., 2016, 6, 28900, DOI: $10.1038 /$ srep28900.

19 A. Housni, M. Ahmed, S. Liu and R. Narain, J. Phys. Chem $C, 2008,112,12282-12290$, DOI: 10.1021/jp803890a.

20 M. S. Bakshi, H. Kaur, T. S. Banipal, N. Singh and G. Kaur, Langmuir, 2010, 26, 13535-13544, DOI: 10.1021/la101701f.

21 L. D. Kong, J. R. Qiu, W. J. Sun, J. Yang, M. W. Shen, L. Wang and X. Y. Shi, Biomater. Sci., 2017, 5, 258-266, DOI: $10.1039 / \mathrm{c} 6 \mathrm{bm} 00708 \mathrm{~b}$.

22 M. S. Bakshi, P. Thakur, G. Kaur, H. Kaur, T. S. Banipal, F. Possmayer and N. O. Petersen, Adv. Funct. Mater., 2009, 19, 1451-1458, DOI: 10.1002/adfm.200801212.

23 P. Khullar, V. Singh, A. Mahal, P. N. Dave, S. Thakur, G. Kaur, J. Singh, S. S. Kamboj and M. S. Bakshi, J. Phys. Chem. C, 2012, 116, 8834-8843, DOI: 10.1021/jp300585d.

24 Y. Ding, L. Shi and H. Wei, J. Mater. Chem. B, 2014, 2, 8268-8291, DOI: $10.1039 /$ C4TB01235F.

25 X. Wang, W. Cao, Q. Xiang, F. Jin, X. Peng, Q. Li, M. Jiang, B. Hu and X. Xing, Mater. Sci. Eng., C, 2017, 76, 886-896, DOI: 10.1016/j.msec.2017.03.192.

26 T. Zhang, P. Zhou, Y. Zhan, X. Shi, J. Lin, Y. Du, X. Li and H. Deng, Carbohydr. Polym. 2015, 117, 687-693, DOI: 10.1016/j.carbpol.2014.10.064.

27 B. Zhou, Y. Li, H. Deng, Y. Hu and B. Li, Colloids Surf., B, 2014, 116, 432-438, DOI: 10.1016/j.colsurfb.2014.01.016.

28 N. G. Balabushevitch, E. P. Zimina and N. I. Larionova, Biochemistry, 2004, 69, 763-769, DOI: $10.1023 / \mathrm{B}$ : BIRY.0000040201.63380.90.

29 N. G. Balabushevitch, G. B. Sukhorukov, N. A. Moroz, D. V. Volodkin, N. I. Larionova, E. Donath and 
H. Mohwald, Biotechnol. Bioeng., 2001, 76, 207-213, DOI: 10.1002/bit.1184.

30 M. K. Ram, P. Bertoncello, H. Ding, S. Paddeu and C. Nicolini, Biosens. Bioelectron., 2001, 16, 849-856, DOI: 10.1016/S0956-5663(01)00208-1.

31 W. Kong, X. Zhang, M. L. Gao, H. Zhou, W. Li and J. C. Shen, Macromol. Rapid Commun., 1994, 15, 405-409, DOI: $10.1002 /$ marc.1994.030150503.

32 F. Caruso, H. Fiedler and K. Haage, Colloids Surf., A, 2000, 169, 287-293, DOI: 10.1016/S0927-7757(00)00443-X.

33 Y. Lvov, A. A. Antipov, A. Mamedov, H. Möhwald and G. B. Sukhorukov, Nano Lett., 2001, 1, 125-128, DOI: 10.1021/nl0100015.

34 F. Caruso and C. Schüler, Langmuir, 2000, 16, 9595-9603, DOI: $10.1021 / \mathrm{la} 000942 \mathrm{~h}$.

35 M. Onda, K. Ariga and T. Kunitake, J. Biosci. Bioeng, 1999, 87, 69-75, DOI: 10.1016/S1389-1723(99)80010-3.

36 E.-C. Wurster, A. Elbakry, A. Göpferich and M. Breunig, Methods Mol. Biol., 2013, 948, 171-182, DOI: 10.1007/978-162703-140-0_12.

37 A. Elbakry, A. Zaky, R. Liebl, R. Rachel, A. Goepferich and M. Breunig, Nano Lett., 2009, 9, 2059-2064, DOI: 10.1021/ nl9003865.

38 S. Ganguli, K. Yoshimoto, S. Tomita, H. Sakuma, T. Matsuoka, K. Shiraki and Y. Nagasaki, J. Am. Chem. Soc., 2009, 131, 6549-6553, DOI: 10.1021/ja900786z.
39 T. Matsuoka, S. Tomita, H. Hamada and K. Shiraki, J. Biosci. Bioeng., 2007, 103, 440-443, DOI: 10.1263/jbb.103.440.

40 C. Pérez and K. Griebenow, Biotechnol. Lett., 2000, 22, 1899-1905, DOI: 10.1023/A:1005645810247.

41 S. Venkataramani, J. Truntzer and D. R. Coleman, J. Pharm. Bioallied Sci., 2013, 5, 148, DOI: 10.4103/0975-7406.111821.

42 D. M. Eby, N. M. Schaeublin, K. E. Farrington, S. M. Hussain and G. R. Johnson, ACS Nano, 2009, 3, 984994, DOI: $10.1021 / \mathrm{nn} 900079 \mathrm{e}$.

$43 \mathrm{P}$. Garidel and H. Schott, Fourier-Transform Midinfrared Spectroscopy for Analysis and Screening of Liquid Protein Formulations. Part 2: Detailed Analysis and Applications, BioProcess International, 2006.

44 F. Dousseau and M. Pezolet, Biochemistry, 1990, 29, 87718779, DOI: $10.1021 /$ bi00489a038.

45 J. L. R. Arrondo, A. Muga, J. Castresana and F. M. Goñi, Prog. Biophys. Mol. Biol., 1993, 59, 23-56, DOI: 10.1016/ 0079-6107(93)90006-6.

46 P. Eaton, P. Quaresma, C. Soares, C. Neves, M. P. de Almeida, E. Pereira and P. West, Ultramicroscopy, 2017, 182, 179-190, DOI: 10.1016/j.ultramic.2017.07.001.

47 S. Ashraf, M. A. Chatha, W. Ejaz, H. A. Janjua and I. Hussain, Nanoscale Res. Lett., 2014, 9, 565, DOI: 10.1186/ 1556-276X-9-565.

48 S. Di Gioia and M. Conese, Drug Des., Dev. Ther, 2009, 2, 163-188, DOI: 10.2147/DDDT.S2708. 

Doctoral Thesis: Electronic tongue technology applied to the analysis of grapes and wines

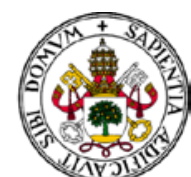

\section{CHAPTER 4}

CONCLUSIONS 

This chapter provides the conclusions extracted from the experimental results that support the achievement of the research objectives proposed for this dissertation relative to the electronic tongue technology applied to the analysis of grapes and wines.

1. Multisensor systems based on voltammetric sensors modified with phthalocyanines are a good alternative to analyze grapes and wines. Carbon paste electrodes (CPES) based on phthalocyanines can be considered ideal for industrial applications because they are cheap and can be easily recovered by cleaning the surface with a filter paper. Sensors prepared by means of nanostructured films of LB or LbL are more sophisticated, but imply a high cost; however, they do improve the sensitivity of the sensors. Finally, biosensors can introduce a degree of specificity very useful for analyzing specific compounds of interest. In the case of the wine sector, this can be very interesting for monitoring the quality of grapes and wines.

2. An electronic tongue based on CPEs modified with metal oxide nanoparticles has been successfully used to monitor the phenolic maturity of grapes. Although oenologists tend to evaluate the sugar content and acidity to estimate the optimal ripening date, the phenolic content is a very important parameter that must be considered at the same level because it can strongly affect the final organoleptic properties of wines. The electrochemical sensors showed an interesting cross-selectivity and improved the limits of detection due to the different electrocatalytic properties provided by metal oxide nanoparticles. The electronic tongue has been shown to be very useful for establishing a better accuracy of the optimal harvesting date and it can therefore be applied as a supplementary tool to classical analytical techniques.

3. A sensor system based on CPEs modified with the skin of grapes has been successfully used to evaluate the phenolic maturity of grapes. The use of grape skin as the sensitive layer provides an easy and direct method to assess their phenolic maturity. However, the correlations between the chemical parameters obtained by traditional methods and the data obtained with the skin sensors were not so good as expected. In this sense, such factors as the skin properties of each variety, as well as environmental factors, can affect the degree of correlation.

This study is considered an original approach for monitoring the phenolic maturity, although some issues must be highlighted: 1) The phenolic maturation process of the vintage and the structural and mechanical skin grape properties are essential factors in finding good 
correlations between the responses provided by skin sensors and the chemical data measured by classical methods; and 2) the preparation of the sensors is a task that must be carried out carefully when peeling the skin off the grapes.

4. Sensors based on Ppy and Ppy/AuNP films evidenced that many factors can influence their conductivity and electrochemical responses, such as the electrodeposition technique used to prepare Ppy films, the way of incorporating AuNPs into the polymeric matrix, the importance of the substrate, as well as the electrolyte solution. The incorporation of AuNP films increased the conductivity of the films and provided electrochemical responses with higher intensities that are more reversible. The method used to introduce the AuNPs did not have a strong influence. However, the concentration of AuNPs was slightly higher when using the cogeneration method, as the SEM images showed. The electrocatalytic activity of the films was improved when using platinum substrate and chronopoentiometry. However, the limits of detection obtained with stainless steel substrate were similar to those obtained with films deposited onto Pt. Therefore, SS is a good substrate in the absence of $\mathrm{KCl}$ or similar, for implementation as a low priced electrochemical sensor. Based on this research, a voltammetric bioET, based on Ppy and Ppy/AuNP films, that is able to predict wine quality values of interest from the data obtained in musts has successfully been developed. The electrochemical sensors are stable during data acquisition, but they are of a single use, as must and wines are complex solutions with components (sugars, proteins and others) that adhere to the sensor surface. Additionally, the bioET was able to discriminate musts and wines in accordance with their chemical parameters: the Brix degree and TPI for musts and the alcoholic degree and TPI for wines.

Phenolic compounds are important components of wines as they can strongly influence their final organoleptic properties. As time is a critical factor in the food industry, the development of this kind of system is clearly necessary to develop new analytical tools that can determine quality features in wines from the beginning of the vinification process, through analyses of the selected grapes for the elaboration of wine.

5. Sensors and biosensors based on PEDOT:PSS films have been shown to have good qualities for being used in the technology of electronic tongues. Moreover, the combination of the conducting polymer with a secondary electrocatalytic component improved the electrochemical responses and limits of detection for di-phenols and, furthermore, the enzymatic sensors provided higher intensities in the responses, lower limits of detection and 
a better charge transfer between the analyte and the electrode surface than non-biosensors. In addition, the spin-coating technique is an easy and simple way to manufacture sensors based on conducting polymers in a short time.

An impedimetric electronic tongue based on the conducting polymer PEDOT:PSS has shown different responses in the EIS experiments carried out on red wines, providing a crossselectivity. They were successfully used as sensing units in an impedimetric electronic tongue. The new feature extraction strategies of selecting parameters from the Nyquist and Bode plots, as well as using the parameters from the equivalent electric circuit, have improved the discrimination of red wines, which could not be achieved with the classical methodology of selecting the resistance and capacitance data at a fixed frequency. The excellent capability of discrimination was similar to those obtained with voltammetric and/or potentiometric tongues. Moreover, the data obtained with the impedimetric electronic tongue can be used to create regression models able to predict the quality parameters of interest in wines.

6. EQCM sensor arrays based on LbL films of metal phthalocyanines have successfully been applied to the discrimination of grape samples of different varieties. The EQCM sensors have provided different information related to the phenolic and sugar content, mainly redox processes, which can be observed in voltammetric measurements; whilst massograms provided information concerning the sugar content. Therefore, this kind of devices can be of interest in the analysis of grape juices and wines, since, simultaneously and in one single experiment, interesting information can be obtained about compounds with different natures that affect the quality of grapes and wines. Moreover, results based on the N-PLS analysis evidenced that it might be possible to establish prediction models able to determine the phenolic content and sugar parameters from the data obtained with the EQCM sensor array in grape samples, which is also very important for monitoring the quality of the grapes.

7. Finally, a protocol has been established to obtain the in situ synthesis and stabilization of lysozyme modified gold nanoparticles where interaction between them is a covalent bond. This protocol does not need the use of activators and could be extended and optimized to create strong interactions between other enzymes and the metal nanoparticles for electrochemical sensing purposes. Therefore, this work is of interest for improving the immobilization of the enzymes tyrosinase, laccase and glucose oxidase, which are usually implemented in our sensors to improve their specificity for phenols and sugars. 
Although several research works have achieved the encapsulation of lysozyme by different strategies, not all of them verify that the lysozyme is still active after its assembly by means of an enzymatic and/or antimicrobial assay. Some of these works only report the presence of the enzyme by means of spectroscopy techniques. Here, we have demonstrated that the enzymatic activity of lysozyme remained intact after the encapsulation of the protein.

As final conclusions, the research work developed during this dissertation demonstrates that:

a) Electronic and bioelectronics tongues developed here are valid and reliable alternatives to analyze grapes and wines for quality purposes, allowing us to analyze such parameters of interest as the phenolic content, sugar or alcoholic degree, among others, which are very important for the organoleptic characteristics of wines.

b) Many electrocatalitic materials have been used and combined, such as AuNPs, metallophthalocyanines, conducting polymers and metal oxide nanoparticles, to modify electrochemical sensors, improving their performance with high reproducibitity, low limits of detection and high specificity, etc.

c) Biosensors based on different enzymes of interest have been developed to study the phenolic and sugar content in grapes and wines and we have implemented these biosensors in bioelectronics tongues. Moreover, biosensors that include specific enzymes have been shown to significantly improve the electrochemical responses of the non-enzymatic sensors.

c) A great variety of methodologies for electrochemical sensor preparation have been implemented, including simple (carbon paste, spin-coating, electrodeposition) and sophisticated (Layer by Layer) techniques.

d) Electronic tongue technology has allowed us to discriminate between grapes of different varieties and wines with different characteristics, as well as wines with different Denomination of Origin, etc.

e) The phenolic ripening of grapes can be monitored using multisensor systems to predict the optimal harvesting time, which is of great importance for wine growers. In the near future, these electronic devices could be applied in situ instead of carrying out lengthy and more expensive experiments in the laboratory. 
f) The electrochemical responses obtained when analyzing grapes can be used to predict the quality parameters of future wines using appropriate chemometric tools for data processing.

g) A new strategy to covalently bond enzymes to gold nanoparticles has been explored that was easy to obtain from the lysozyme protein and that can be applied to other enzymes of interest for sensing applications in the food industry. 

Doctoral Thesis: Electronic tongue technology applied to the analysis of grapes and wines

ANNEXES 

Doctoral Thesis: Electronic tongue technology applied to the analysis of grapes and wines

Annex A: Resumen en castellano 



\section{1.- Introducción}

La Tesis Doctoral titulada "Tecnología de la lengua electrónica aplicada al análisis de uvas y vinos" ha sido desarrollada en el grupo de sensores UVaSens bajo la dirección de las Profesoras María Luz Rodríguez Méndez, del Departamento de Química Inorgánica y Química Física de la Escuela de Ingenieros Industriales, y Ana Cristina García Cabezón, del Departamento de Ciencia de Materiales e Ingeniería Metalúrgica de la Escuela de Ingenieros Industriales, ambas de la Universidad de Valladolid (España). Además, la autora de esta tesis ha realizado una estancia de investigación de 4 meses en la Universidad Estatal de Misuri (Missouri State University, Springfield, EE.UU.) bajo la supervisión del Profesor Adam Wanekaya para optar a la Mención Internacional de doctorado.

Esta disertación contiene trabajos experimentales que se han publicado en revistas internacionales donde se ha estudiado el uso de sensores y biosensores implementados como redes de electrodos en lenguas electrónicas y bioelectrónicas para analizar parámetros relacionados con la calidad de vinos y uvas. Para la construcción de estas redes de sensores se han utilizado y/o combinado diferentes y novedosos materiales electrocatalíticos. Las muestras de vino y uva utilizadas en este estudio han sido proporcionadas por las bodegas "Cooperativa de Cigales" e "Instituto Tecnológico Agrario de Castilla y León (ITACyL)", ambas situadas en la región de Castilla y León (Valladolid, España), y por el "Centro Enológico de Haro" situado en La Rioja (Haro, España). Los parámetros químicos de las muestras fueron analizados en el laboratorio del grupo UVaSens y en el ITACyL siguiendo la normativa internacional (OIV, The International Organisation of Vine and Wine).

El desarrollo de nuevos métodos de análisis para caracterizar los alimentos es de vital importancia para mejorar los actuales sistemas de control de calidad de los productos alimenticios. Dentro de este campo, el concepto de lengua electrónica (ETs o e-tongues) ha crecido rápidamente en los últimos años debido a su gran potencial. Estos dispositivos se basan en sensores electroquímicos combinados con análisis de datos multivariantes. De acuerdo con la IUPAC (Unión Internacional de Química Pura y Aplicada), una lengua electrónica es un sistema multisensor, que consiste en un número de sensores de baja selectividad y utiliza procedimientos matemáticos avanzados para el procesamiento de señales basados en el reconocimiento de patrones (PARC) y/o análisis multivariante [redes neuronales artificiales (RNA), análisis de componentes principales (PCA), etc.]. Por lo tanto, las ETs son sistemas holísticos que proporcionan información global y cualitativa acerca de la muestra en lugar de datos cuantitativos 
acerca de compuestos específicos. Sin embargo, si la matriz de datos obtenida por estos sistemas se analiza con herramientas de procesamiento quimiométrico adecuadas, se podría extraer información descriptiva o predictiva de parámetros específicos. Existe un término más reciente en el campo de las lenguas electrónicas, ampliamente denominado lengua bioelectrónica (bioET), que incluye el uso de uno o varios biosensores implementados en las ETs.

Durante esta investigación se han aplicado ETs y bioETs para estudiar las uvas tintas y los vinos con el fin de predecir mejor el momento óptimo de la vendimia de uvas, así como los parámetros de calidad de interés en los vinos.

El vino es la bebida alcohólica obtenida de la fermentación de la uva y es uno de los alimentos más antiguo conocido desde hace más de 8000 años. El origen de la viticultura se remonta a los años 6000-5000 a.C. en Georgia e Irán. Desde entonces, la cultura vitivinícola sigue siendo prácticamente la misma: el vino moderno es muy similar al que elaboraban nuestros antepasados, y las prácticas modernas de viticultura y enología todavía se refieren a las practicadas por los antiguos griegos, que fueron conservadas y luego evolucionadas durante el Imperio Romano.

La matriz del vino presenta una de las composiciones químicas más complejas con más de 800-1000 compuestos volátiles que van desde alcoholes, ésteres, ácidos, cetonas, éteres, aldehídos, terpenos, lactonas, compuestos fenólicos, etc., procedentes de la uva y/o que se forman durante el proceso de fermentación, con concentraciones muy variadas. La abundancia de estos compuestos depende de muchos factores: variedad y origen de la uva, prácticas vitivinícolas (mezcla, envejecimiento, levadura utilizada durante la fermentación), tratamiento de la producción, almacenamiento y exposición al oxígeno, etc.

Los análisis rutinarios que se llevan a cabo en los laboratorios enológicos, desde la vendimia de la uva hasta el producto final embotellado, incluyen una serie de cromatografías, así como técnicas más tradicionales (espectroscopía, fotometría, ensayos enzimáticos, etc.). Estas técnicas analíticas se combinan para obtener un estudio profundo de la composición de los vinos, lo que da lugar a análisis muy costosos, que consumen mucho tiempo y exigen personal altamente cualificado. Por lo tanto, es necesario desarrollar nuevas tecnologías que nos permitan obtener una información global de las muestras en lugar de información de componentes específicos. Desde el punto de vista analítico, el análisis del vino es una tarea difícil debido a la complejidad de la mezcla y, además, pequeñas diferencias en la concentración de ciertos 
compuestos dan lugar a vinos con características organolépticas completamente diferentes. Además, la sinergia entre grupos de compuestos tiene a menudo una mayor influencia en las características organolépticas que los compuestos individuales.

Tradicionalmente, los viticultores han estimado la fecha óptima de vendimia evaluando el contenido de azúcar y la acidez de las uvas, pero otros compuestos como los fenoles son tan importantes como los azúcares y ácidos presentes en las uvas debido a que la composición fenólica afecta directamente a las características organolépticas (sabor, color, textura, olor) de los vinos.

Los sistemas ETs pueden convertirse en una alternativa o complemento a los métodos tradicionales de análisis de vino y uva. Además, estos sistemas pueden ser utilizados no sólo en los laboratorios analíticos sino también in situ por los agricultores y viticultores en los viñedos como una herramienta de control de calidad.

Los sensores utilizados en lenguas electrónicas son principalmente ópticos, electroquímicos y gravimétricos. En aplicaciones para el análisis de alimentos, los más utilizados son los sensores electroquímicos (potenciométricos, amperométricos, voltamperométricos e impedimétricos) debido a su sencilla instrumentación, alta sensibilidad, bajo tiempo de respuesta y facilidad de operación.

Dentro de la investigación que aplica lenguas electrónicas, existe un especial interés en implementar materiales electrocatalíticos en los sensores electroquímicos que mejore las prestaciones de estos dispositivos, especialmente su sensibilidad y especificidad. En este sentido, los materiales utilizados para los sensores electroquímicos se pueden clasificar como: 1) materiales para el electrodo y el sustrato de soporte, 2) materiales para mejorar las prestaciones electroanalíticas, 3) materiales para la inmovilización de elementos de reconocimiento biológico y 4) elementos biológicos, siendo estos dos últimos aplicables a los biosensores electroquímicos. La elección de estos materiales sensibles para su aplicación en lenguas electrónicas o bioelectrónicas es crucial para obtener un alto rendimiento. En función de sus propiedades, estos materiales pueden actuar de forma diferente en un sistema electroquímico aunque las funciones básicas de los mismos pueden clasificarse principalmente como: 1) mejora de la transferencia de electrones, 2) catálisis de reacciones electroquímicas, 3) inmovilización de biomoléculas, 4) etiquetado de biomoléculas y 5) actuar como reactante. En los trabajos de investigación desarrollados durante la presente Tesis Doctoral se han utilizado diferentes materiales sensibles como son ftalocianinas, nanopartículas metálicas, materiales a base de carbono y polímeros 
conductores para mejorar la sensibilidad de los sensores y, también, se ha trabajado con enzimas de interés para la detección de fenoles y azúcares con el fin de mejorar la especificidad de los sensores.

Por otro lado, los sensores se pueden modificar químicamente con materiales electrocatalíticos mediante un gran variedad de técnicas, abarcando la preparación de sensores clásicos, preparados mediante técnicas sencillas (electrodos de pasta de carbono, casting, electrodeposición, etc.), hasta la implementación de técnicas más sofisticadas y costosas para fabricar sensores nanoestructurados mediante técnicas como SAM (Self-assembled Monolayer, SAM), Layer by Layer (LbL) o Langmuir-Blodgett (LB).

\section{2.- Objetivos}

El objetivo principal de esta investigación ha sido el desarrollo de nuevos sensores electroquímicos utilizando materiales electrocatalíticos que mejoren las prestaciones en cuanto a la sensibilidad de los sensores. Estos sensores fueron después implementados en lenguas electrónicas y bioelectrónicas para el estudio, principalmente, de antioxidantes y polifenoles presentes en la uva y el vino.

Los sensores que se han desarrollado en este trabajo de investigación han sido tanto de tipo no-nanoestructurados como nanoestructurados, para lo cual la nanotecnología ha jugado un papel muy importante en su fabricación mediante el uso de diferentes técnicas de modificación de superficies. Además, también se han combinado algunos de los materiales electrocatalíticos utilizados para así estudiar el efecto sinérgico entre ellos y la mejora en la respuesta de los sensores. Los dispositivos de ETs y/o bioETs se han utilizado para analizar muestras reales de vino y uva. Mediante los análisis estadísticos apropiados, aplicados los datos multivariantes obtenidos de los análisis, se ha discriminado entre las muestras analizadas y se han establecido correlaciones entre los datos obtenidos con los dispositivos ETs y bioETs y los parámetros químicos obtenidos por el análisis tradicional.

Los objetivos específicos del trabajo de investigación han sido:

1. Diseñar y construir lenguas electrónicas y bioelectrónicas específicamente dedicadas al análisis de calidad de vinos y uvas mediante sensores electroquímicos modificados químicamente. 
2. Mejorar el rendimiento de los sensores mediante: (a) nuevos materiales electrocatalíticos, (b) sensores basados en películas nanoestructuradas, (c) combinación de diferentes materiales electrocatalíticos para aumentar la capacidad electroquímica de los sensores y (c) la introducción de enzimas para mejorar la especificidad de los sensores para detectar compuestos fenólicos y/o azúcares.

3. Implementar diferentes técnicas de modificación de superficie como, por ejemplo, electrodeposición, spin-coating y/o Layer by Layer con el fin de mejorar el rendimiento de los sensores, tales como su sensibilidad, reproducibilidad y/o vida útil.

4. Aplicar diferentes técnicas electroquímicas de análisis (voltametría cíclica, espectroscopía de impedancias, microbalanza electroquímica de cristal de cuarzo, etc.) para evaluar su versatilidad.

5. Estudiar nuevos métodos para unir enzimas con nanomateriales (ej. nanopartículas metálicas) mediante interacciones fuertes de tipo covalente.

6. Validar la lengua electrónica como herramienta de control durante la maduración fenólica de la uva, desde el momento de la maduración hasta la fecha de óptima de maduración.

7. Evaluar la capacidad de las lenguas electrónicas en la autenticación de muestras de uva y vino.

8. Establecer modelos de correlación válidos entre los resultados obtenidos con la lengua electrónica y/o bioelectrónica en muestras reales de vino y uva con los parámetros químicos medidos con métodos analíticos tradicionales.

9. Evaluar la capacidad del sistema para predecir parámetros de calidad en vinos a partir de los datos obtenidos del análisis de uvas utilizadas para elaborar los correspondientes vinos.

\section{3.- Metodología y resultados}

Para llevar a cabo los objetivos propuestos se han realizado diferentes trabajos de investigación en el grupo UVaSens donde se han utilizado diferentes materiales electrocatalíticos, técnicas de preparación de sensores y técnicas electroquímicas de análisis. Además, se han llevado a cabo diferentes análisis en muestras de uva y vino para validar los sistemas de ETs y bioETs. 
UVaSens es un grupo multidisciplinar experto en el desarrollo de sensores electroquímicos, principalmente voltamperométricos, para su aplicación en lenguas electrónicas. El trabajo de investigación del grupo incluye la fabricación y caracterización de sensores clásicos basados en CPE, spin-coating o electrodeposición, pero también la preparación de películas delgadas nanoestructuradas utilizando técnicas como Layer by Layer o Langmuir-Blodgett. Además, para la construcción de los sensores, el grupo ha utilizado ampliamente materiales electrocatalíticos como ftalocianinas y bisftalocianinas, tanto en sensores nanoestructurados como no nanoestructurados. El grupo también ha implementado polímeros conductores en los sensores cuando comenzaron a ser aplicados en sensores electroquímicos. Por otro lado, el grupo UVaSens fue uno de los primeros en desarrollar lenguas electrónicas voltamperométricas modificadas basadas en CPE y ftalocianinas para mejorar la respuesta electroquímica. Así, el artículo I que se presenta en esta Tesis, "Multisensor systems based on phthalocyanines for monitoring the quality of grapes", es un review que ofrece una visión general de las lenguas electrónicas basadas en ftalocianinas para el análisis de uvas en el que se discute el uso de sensores y biosensores no nanoestructurados y nanoestructurados.

Los sistemas multisensor basados en sensores voltamperométricos modificados con ftalocianinas son una buena alternativa para analizar uvas y vinos. Los electrodos de pasta de carbono (CPEs) basados en ftalocianinas pueden considerarse ideales para aplicaciones industriales porque son baratos y pueden recuperarse fácilmente limpiando la superficie con un papel de filtro. Los sensores preparados mediante películas nanoestructuradas LB o LbL son más sofisticados implicando un alto coste, sin embargo, mejoran la sensibilidad de los sensores ya que ofrecen una mayor relación superficie-volumen. Finalmente, los biosensores basados en sensores modificados con enzimas pueden introducir un grado de especificidad muy útil para analizar compuestos específicos de interés, como pueden ser los azúcares y polifenoles en el caso del vino y la uva. Por lo tanto, los biosensores pueden ser muy interesantes para el control de la calidad de las uvas y los vinos.

Los artículos II a VII se centran en sensores electroquímicos preparados mediante técnicas clásicas como son CPEs, electrodeposición y spin-coating. En el artículo II, "Monitoring the phenolic ripening of red grapes using a multisensor system based on metal-oxide nanoparticles", el grafito se mezcló con diferentes nanopartículas de óxidos metálicos para construir una red de sensores químicamente modificados. Esta red de sensores se ha utilizado por primera vez como lengua electrónica voltamperométrica para monitorizar la madurez de las uvas en función de su madurez fenólica. Además, las respuestas voltamétricas obtenidas con 
esta lengua electrónica mostraron una buena correlación con el contenido fenólico de las uvas. Con estos sensores se consiguió una interesante selectividad cruzada en las respuestas y límites de detección en compuestos fenólicos del mismo orden que los obtenidos con otros sensores más sofisticados y nanoestructurados, todo ello gracias a las propiedades electrocatalíticas que proporcionan las nanopartículas de óxidos metálicos. Estos sensores tienen la ventaja de que pueden recuperarse fácilmente limpiando la superficie con un papel de filtro, lo que los hace muy apropiados para aplicaciones industriales.

En el artículo III, "A different approach for the analysis of grapes: Using the skin as sensing element", se ha desarrollado un trabajo original, nunca antes propuesto, en el que se ha sustituido el uso de un material electrocatalítico para modificar el electrodo CPE por la propia piel de la uva. Para ello, la piel de la uva se depositó sobre la superficie de un electrodo CPE con un contacto íntimo entre el grafito y la cara interna de la piel. Teniendo en cuenta que los hollejos de la uva contienen fenoles que serán liberados a la pulpa durante el proceso de maduración e influirán en el color, sabor y calidad de las uvas y los vinos, se pensó en estudiar la madurez de la uva directamente mediante el análisis de la piel. Las respuestas voltamétricas de los sensores durante la maduración de las uvas mostraron cambios en las intensidades y los picos asociados a los compuestos polifenólicos y se ha podido monitorizar la madurez fenólica de las uvas. Sin embargo, algunos aspectos deben ser mejorados y tomados en cuenta para obtener mejores correlaciones entre las respuestas de los sensores y los parámetros químicos obtenidos por los métodos tradicionales. En este sentido, factores como las propiedades de la piel de cada variedad y los factores ambientales pueden afectar el grado de correlación. Además, en este caso, estos sensores son de un solo uso, ya que la limpieza de la superficie con un papel de filtro destruiría la piel despositada sobre la superficie del electrodo.

En los artículos IV a VII se han utilizado polímeros conductores en la preparación de los sensores y/o biosensores. Así, en el artículo IV, "Electrochemical behavior of polypyrrol/AuNP composites deposited by different electrochemical methods: sensing properties towards catechol", se ha electrodepositado una película de polipirrol sobre sustratos de platino y acero inoxidable y, también, se han incorporado AuNPs en las películas para mejorar el efecto electrocatalítico de los sensores. Los sensores preparados se caracterizaron utilizando SEM, EIS y voltamperometría cíclica. Posteriormente, se aplicaron para analizar el polifenol catecol, que es un grupo fenólico de interés en el proceso de oxidación del vino. Los resultados obtenidos con los sensores basados en películas de Ppy y Ppy/AuNPs evidenciaron que muchos factores pueden influir en su conductividad y respuesta electroquímica, 
tales como la técnica de electrodeposición utilizada para preparar las películas de Ppy (cronopotenciometría o cronoamperometría), el método de incorporación de AuNPs en la matriz polimérica, la importancia del sustrato, así como el medio electrolito utilizado para realizar las medidas electroquímicas. La incorporación AuNPs en la matriz polimérica aumenta la conductividad de las películas y proporciona respuestas electroquímicas con intensidades más altas y más reversibles. La influencia del método utilizado para introducir las AuNPs, ya sea previamente sintetizadas y mezcladas con el precursor del polímero antes de realizar la electrodeposición (atrapamiento) o mediante la síntesis in situ durante la electrodeposición a partir de la sal precursora (cogeneración), no influye significativamente en la respuesta electroquímica. Sin embargo, la concentración de AuNPs es ligeramente superior cuando se utiliza el método de cogeneración, como han demostrado las imágenes SEM. La actividad electrocatalítica de las películas mejora cuando se utiliza un sustrato de platino y la técnica de cronopoentiometría. Los límites de detección obtenidos con sustrato de acero inoxidable son similares a los obtenidos con películas depositadas sobre platino y, por tanto, supone una alternativa de bajo coste a los sustratos basados en metales nobles utilizados en sensores electroquímicos.

Los sensores desarrollados en el artículo IV se han utilizado en el trabajo de investigación que se expone el artículo V, "Analysis of musts and wines by means of a bioelectronic tongue based on tyrosinase and glucose oxidase using polypyrrole/gold nanoparticles as the electron mediator". En este trabajo se ha implementado, por primera vez, una lengua bioelectrónica voltamétrica para el análisis de mostos y los vinos elaborados a partir de ellos. La lengua bioelectrónica consistió en sensores de Ppy/AuNPs donde se ha depositado una enzima sobre el sustrato mediante la téncia de drop-casting y entrecruzamiento con glutaraldehído. De este modo, se desarrollaron biosensores a partir de los sensores de Ppy/AuNPs que mejor respuesta electroquímica ofrecieron en el trabajo de investigación previo. Las enzimas que se han utilizado en este estudio fueron tirosinasa y glucosa oxidasa, para mejorar la especificidad de los sensores a los fenoles y azúcares, respectivamente. La novedad de este trabajo radica en el hecho de que, nunca antes, se habían utilizado los datos voltamétricos obtenidos en uvas para predecir parámetros de calidad de los vinos elaborados con dichas uvas. Los sensores electroquímicos ofrecieron respuestas estables en mostos y vinos, pero son de un solo uso debido a que las muestras son mezclas muy complejas con componentes que se adhieren a la superficie del sensor (azúcares, proteínas y otros). En este trabajo se han utilizado las técnicas multivariantes de PARAFAC y PCA para discriminar entre 
las muestras analizadas a partir de los datos obtenidos con la lengua bioelectrónica. Se han discriminado los mostos de diferente variedad de uva de acuerdo con su contenido en azúcares y contenido polifenólcio y, también, se discriminaron vinos con distinto grado alcohólico y contenido polifenólico. Además, el análisis con PLS-1 permitió establecer modelos de correlación aceptables con los parámetros químicos de azúcar, grado alcohólico y contenido polifenólico y, finalmente, se ha podido predecir el contenido polifenólico y el grado alcohólico de muestras de vino a partir de los datos voltamétricos obtenidos en los mostos de uva que se utilizaron para elaborar dichos vinos. Este resultado es muy interesante, ya que los viticultores podrían conocer cuál será la calidad de sus vinos antes de su elaboración, simplemente a partir de los datos obtenidos en las uvas de vendimia.

Los artículos VI y VII se basan en el polímero conductor PEDOT:PSS. Así, en el artículo VI, "Layered composites of PEDOT/PSS/nanoparticles and PEDOT/PSS/phthalocyanines as electron mediators for sensors and biosensors", se prepararon sensores modificados con PEDOT:PSS mediante la técnica de spin-coating sobre sustrato de ITO. En una segunda etapa, se han modificado los sensores de PEDOT:PSS incorporado otros materiales electrocatalíticos como AuNPs, ftalocianina de cobre y bisftalocianina de lutecio, con el fin de mejorar el rendimiento electroquímico de los sensores. Además, las enzimas tirosinasa y lacasa se depositaron en la superficie de los electrodos utilizando también la técnica de spin-coating. Estos sensores y biosensores han mostrado excelentes límites de detección y reproducibilidad hacia el polifenol catecol y su isómero hidroquinona. Los sensores enzimáticos proporcionaron mayores intensidades en las respuestas, mejores límites de detección y una mejor transferencia de carga entre el analito y la superficie del electrodo que los no-enzimáticos.

Tras los resultados obtenidos con los sensores basados en PEDOT:PSS, en el artículo VII, "Impedimetric electronic tongue based on nanocomposites for the analysis of red wines. Improving the variable selection method", se han aplicado estos sensores en lengua electrónica impedimétrica para la autentificación de vinos elaborados con diferentes variedades de uva y de diferentes Denominaciones de Origen. Esta aplicación es novedosa ya que sólo existe un trabajo de investigación que reporte el uso de una lengua impedimétrica para el análisis de vinos. Además, el uso de lenguas electrónicas impedimétricas aplicadas al análisis de alimentos es bastante escaso. Los sensores basados en PEDOT:PSS han mostrado diferentes respuestas en los experimentos EIS hacia los vinos tintos proporcionando una selectividad cruzada y, por lo tanto, se ha obtenido una buena discriminación entre los diferentes vinos analizados. 
En este trabajo, además, se ha llevado a cabo una nueva estrategia de extracción de variables a partir de parámetros obtenidos de los gráficos de Nyquist y Bode, así como el uso de parámetros del circuito eléctrico equivalente, que han mejorado notablemente la discriminación de los vinos tintos, resultado que no se ha obtenido mediante la metodología clásica que utiliza los datos de resistencia y capacitancia a una frecuencia fija. La excelente capacidad de discriminación fue similar a la obtenida con lenguas voltamperométricas y/o potenciométricas. Además, los datos obtenidos con la lengua electrónica impedimétrica se han utilizado para crear modelos de regresión capaces de predecir los parámetros de calidad de interés en los vinos.

En los artículos VIII y IX se ha aplicado la nanociencia y la nanotecnología, concretamente, la tecnología de Layer by Layer (LbL), aunque con dos propósitos diferentes. En el artículo VIII, "An electrochemical quartz crystal microbalance multisensor system based on phthalocyanine nanostructured films: Discrimination of musts", se depositaron películas LbL nanoestructuradas de ftalocianinas metálicas en sensores de microbalanza de cristal de cuarzo (QCM) para su uso como lengua electrónica voltamétrica y másica. Estos cristales están recubiertos por un electrodo soporte que consiste en una lámina de platino. En concreto, se utilizaron diferentes ftalocianinas sulfónicas para obtener una mayor selectividad cruzada en las respuestas voltamétricas. Esta lengua electrónica se ha utilizado por primera vez en el análisis de alimentos para registrar de manera simultáneamente datos voltamperométricos y cambios de masa que ocurren durante los procesos de oxidación y reducción en mostos de uva. Se analizaron y discriminaron mostos de uvas de diferentes variedades en función de su contenido en azúcares y fenoles. Los procesos redox observados en las medidas voltamétricas aportan información sobre el contenido de polifenoles, mientras que los masogramas proporcionan información sobre el contenido de azúcar. La principal ventaja de los sensores basados en QCM sobre los sensores electroquímicos tradicionales es que proporcionan más información (electroquímica y másica) en un solo experimento. Además, en comparación con los trabajos mencionados anteriormente, los sensores basados en nanoestructuras LbL son más sofisticados y proporcionan una mayor relación superficie/volumen, pero siguen siendo fáciles de preparar y económicos. Por lo tanto, este tipo de dispositivos puede ser de gran interés para utilizarse en el análisis de alimentos ya que se puede obtener, simultáneamente y en un solo experimento, información interesante sobre compuestos de diferente naturaleza. Además, mediante el análisis con N-PLS se establecieron modelos de predicción capaces de determinar el contenido fenólico y los parámetros de azúcar a partir de los datos obtenidos con la lengua electrónica basada en sensores QCM, lo que puede ser una alternativa para controlar la calidad de las uvas. 


\section{En el último trabajo, "In situ synthesis, stabilization and activity of protein-modified} gold nanoparticles for biological applications", también se ha utilizado la técnica LbL, si bien en este trabajo se ha utilizado para preparar una nanoestructura que contiene AuNPs y la enzima lisozima. Este trabajo ha sido desarrollado en la Universidad Estatal de Misuri (Springfield, USA) durante una estancia de investigación de 4 meses. Aunque este trabajo no se basa en el desarrollo de sensores para su aplicación en lenguas electrónicas, o incluso en la industria alimentaria, puede extenderse y optimizarse a este campo ya que el ensamblaje que se ha desarrolado se puede aplicar al caso de otras enzimas que sean de interés para la industria de la alimentación como la tirosinasa, lacasa o glucosa oxidasa, que ya han sido utilizados en los trabajos mencionados anteriormente. Aunque en otros trabajos de investigación se ha logrado la encapsulación de la lisozima mediante diferentes estrategias, no todos ellos verifican que la lisozima tenga actividad enzimática después de su ensamblaje mediante un ensayo enzimático y/o antimicrobiano. Algunos de estos trabajos sólo informan de la presencia de la enzima mediante técnicas de espectroscopía. En este trabajo se ha demostrado que la actividad enzimática de la lisozima permanece intacta después de su encapsulación.

\section{4.- Conclusiones}

A continuación, se presentan unas conclusiones generales que apoyan la consecución de los objetivos de investigación propuestos en esta disertación sobre la tecnología de la lengua electrónica aplicada al análisis de uvas y vinos.

a) Las lenguas electrónicas y bioelectrónicas que se han desarrollado son alternativas válidas y fiables para analizar la calidad de uvas y vinos que permite evaluar parámetros de interés tales como el contenido fenólico, azúcar o grado alcohólico, entre otros, muy importantes para las características organolépticas de los vinos.

b) Se han utilizado y combinado muchos materiales electrocatalíticos como AuNPs, ftalocianinas metálicas, polímeros conductores y nanopartículas de óxido metálicos para modificar los sensores electroquímicos y mejorar el rendimiento de los sensores en términos de alta reproducibilidad, bajos límites de detección, alta especificidad, etc.

c) Se han desarrollado biosensores basados en diferentes enzimas de interés para el estudio del contenido fenólico y en azúcares en uvas y vinos y se han implementado estos biosensores en lenguas bioelectrónicas. Además, los biosensores que incluyen enzimas 
específicas han demostrado que mejoran significativamente las respuestas electroquímicas con respecto a los sensores no-enzimáticos.

c) Se han aplicado una gran variedad de metodologías para la preparación de los sensores electroquímicos que incluyen técnicas simples (pasta de carbono, spin-coating, electrodeposición) y otras más sofisticadas (Layer by Layer).

d) Los sistemas de lengua electrónica han permitido discriminar entre uvas y vinos de diferentes variedades y características, vinos con diferentes Denominaciones de Origen, etc.

e) La maduración fenólica de las uvas puede ser monitorizada mediante sistemas multisensor con el fin de predecir el momento óptimo de la vendimia, lo que es de gran importancia para los viticultores que, en un futuro próximo, podrían aplicar estos dispositivos electrónicos in situ en lugar de llevar a cabo experimentos en los laboratorios que requieren de mucho tiempo, personal cualificado y son más costosos.

f) Las respuestas electroquímicas obtenidas en el análisis de las uvas pueden ser utilizadas para predecir los parámetros de calidad de los futuros vinos mediante el uso de un análisis quimiométrico de datos adecuado.

g) Se ha explorado una nueva estrategia para unir covalentemente la enzima lisozima a nanopartículas de oro, fácil de obtener y que puede aplicarse a otras enzimas de interés específicas para evaluar el contenido fenólico y en azúcares.

Dado que el tiempo es un factor crítico en la industria del vino, el desarrollo de este tipo de sistemas basados en lenguas electrónicas puede ser novedoso y de gran interés para desarrollar nuevas herramientas analíticas que permitan determinar la calidad de los vinos desde el inicio del proceso de vinificación, analizando las uvas seleccionadas para la elaboración del vino. De esta forma, los enólogos podrán conocer las características organolépticas y la calidad de los vinos desde la etapa más temprana. 
Doctoral Thesis: Electronic tongue technology applied to the analysis of grapes and wines

\section{Annex B: List of publications}



The co-authored list of papers published during the Doctoral studies is attached below:

C. García-Hernández, A. Freese, M.L. Rodríguez-Méndez, A.K. Wanekaya. In situ synthesis, stabilization and activity of protein modified gold nanoparticles for biological applications. Biomaterials Science 7 (2019) 2511-2519.

doi: 10.1039/c9bm00129h

Impact Factor (2018 JCR): 5.251; Quartil (2018 JCR): Q1, Category: Materials Science, Biomaterials.

C. García-Cabezón, C. García-Hernández, M.L. Rodríguez-Méndez, F. Martín-Pedrosa. A new strategy for corrosion protection of porous stainless steel using polypyrrole films. Journal of Materials Science and Technology (2019) [In Press, Journal Pre-proof]. doi: 10.1016/j.jmst.2019.05.071 Impact Factor (2018 JCR): 5.040; Quartil (2018 JCR): Q1, Category: Metallurgy and Metallurgical Engineering; Materials Science, Multidisciplinary.

C. García-Hernández, C. García-Cabezón, F. Martín-Pedrosa, M.L. Rodríguez-Méndez. Analysis of musts and wines by means of a bio-electronic tongue based on tyrosinase and glucose oxidase using polypyrrole/gold nanoparticles as the electron mediator. Food Chemistry 289 (2019) 751-756.

doi: 10.1016/j.foodchem.2019.03.107

Impact Factor (2018 JCR): 5.399; Quartil (2018 JCR): Q1, Categories: Food Science and Technology; Chemistry, Applied; Nutrition and Dietetics.

C. García-Hernández, C. Salvo Comino, F. Martín-Pedrosa, M.L. Rodríguez-Méndez, C. García-Cabezón. Impedimetric electronic tongue based on nanocomposites for the analysis of red wines. Improving the variable selection method. Sensors and Actuators $B$ 277 (2018) 365-372.

doi: 10.1016/j.snb.2018.09.023

Impact Factor (2018 JCR): 6.393; Quartil (2018 JCR): Q1, Categories: Electrochemistry; Chemistry, Analytical; Instruments and Instrumentation. 
R. Muñoz, C. García-Hernández, C. Medina-Plaza, C. García-Cabezón, J.A. FernándezEscudero, E. Barajas, G. Medrano, M.L. Rodríguez-Méndez. A different approach for the analysis of grapes: Using the skin as sensing element. Food Research International 107 (2018) 544-550.

doi: 10.1016/j.foodres.2018.02.060

Impact Factor (2018 JCR): 3.579; Quartil (2018 JCR): Q1, Category: Food Science and Technology.

C. Salvo-Comino, C. García-Hernández, C. García-Cabezón, M.L. Rodríguez-Méndez. Discrimination of milks with a multisensor system based on layer-by-layer films. Sensors 18 (2018) 2716.

doi: $10.3390 / \mathrm{s} 18082716$.

Impact Factor (2018 JCR): 3.031; Quartil (2018 JCR): Q1, Categories: Instruments and Instrumentation.

C. García-Hernández, C. Medina-Plaza, C. García-Cabezón, Y. Blanco, J.A. FernándezEscudero, E. Barajas-Tola, M.A. Rodríguez-Pérez, F. Martín-Pedrosa, M.L. RodríguezMéndez. Monitoring the phenolic ripening of red grapes using a multisensor system based on metal-oxide nanoparticles. Frontiers in Chemistry 6 (2018) 131.

doi: $10.3389 / f c h e m .2018 .00131$

Impact Factor (2018 JCR): 3.782; Quartil (2018 JCR): Q2, Categories: Chemistry, Multidisciplinary.

R. González-Antón, M.M. Osipova, C. García-Hernández, T.V. Dubinina, L.G. Tomilova, C. García-Cabezón, M.L. Rodríguez-Méndez. Subphthalocyanines as electron mediators in biosensors based on phenol oxidases: Application to the analysis of red wines. Electrochimica Acta 255 (2017) 239-247.

doi: 10.1016/j.electacta.2017.09.168

Impact Factor (2017 JCR): 5.116; Quartil (2017 JCR): Q1, Category: Electrochemistry. 
M.L. Rodríguez-Méndez, J.A. de Saja, R. González-Antón, C. García-Hernández, C. Medina-Plaza, C. García-Cabezón, F. Martín-Pedrosa. Electronic noses and tongues in wine industry. Frontiers in Bioengineering and Biotechnology 4 (2016) 1-12.

doi: 10.3389/fbioe.2016.00081

Impact Factor (2018 JCR): 5.122; Quartil (2018 JCR): Q1, Category: Multidisciplinary Sciences.

M.L. Rodríguez-Méndez, C. García- Hernández, C. Medina-Plaza, C. García-Cabezón, D. Paniagua-Albillos, S. Rodríguez-Sánchez, J.A. de Saja. Electrochemical sensors for the detection of antioxidants. In Frontiers in Bioactive Compounds (Vol. I). Natural sources, physicochemical characterization and applications, Ed. C. Apetrei. Benthan Science Publishers. Sharjah, UAB, 2016, pp. 127-146.

ISBN: 978-1-68108-342-1

M.L. Rodríguez-Méndez, J.A. de Saja, C. Medina-Plaza, C. García- Hernández. Electronic tongues for the organoleptic characterization of wines. In Electronic Noses and Tongues in Food Science, Ed. M.L. Rodríguez-Méndez. Elsevier: Amsterdam, The Netherlands, 2016, pp. 265-273.

ISBN: $978-0-12-800243-8$

M. Sliwinska, C. García-Hernández, M. Koscinski, T. Dymerski, W. Wardencki, J. Namiesnik, M. Sliwinska-Bartkowiak, S. Jurga, C. García-Cabezón, M.L. RodríguezMéndez. Discrimination of apple liqueurs (Nalewka) using a voltammetric electronic tongue, UV-Vis and Raman spectroscopy. Sensors 16 (2016) 1654.

doi: $10.3390 / s 16101654$

Impact Factor (2016 JCR): 2.677; Quartil (2016 JCR): Q1, Category: Instruments and Instrumentation. 
C. García-Hernández, C. García-Cabezón, F. Martín-Pedrosa, J.A. de Saja, M.L. Rodríguez-Méndez. Layered composites of PEDOT/PSS/nanoparticles and PEDOT/PSS/phthalocyanines as electron mediators for sensors and biosensors. Beilstein Journal of Nanotechnology 7 (2016) 1948-1959.

doi: 10.3762/bjnano.7.186

Impact Factor (2016 JCR): 3.127; Quartil (2016 JCR): Q1, Category: Materials Science, Multidisciplinary; Physics, Applied.

M.L. Rodríguez-Méndez, C. García-Hernandez, C. Medina-Plaza, C. García-Cabezón, J.A. de Saja. Multisensor systems based on phthalocyanines for monitoring the quality of grapes. Journal of Porphyrins and Phthalocyanines 20 (2016) 889-894.

doi: $10.1142 / S 1088424616500796$

Impact Factor (2016 JCR): 1.043; Quartil (2016 JCR): Q3, Category: Chemistry, Multidiscipliary.

M.L. Rodríguez-Méndez, C. Medina-Plaza, C. García-Hernández, S. Rodríguez, C. García-Cabezón, D. Paniagua, M.A. Rodríguez-Pérez, J.A. de Saja. Improvement of electrocatalytic effect in voltammetric sensors based on phthalocyanines. Journal of Porphyrins and Phthalocyanines 20 (2016) 413-420.

doi: $10.1142 / S 1088424616500218$

Impact Factor (2016 JCR): 1.043; Quartil (2016 JCR): Q3, Category: Chemistry, Multidiscipliary.

C. García-Hernández, C. Medina-Plaza, C. García-Cabezón, F. Martín-Pedrosa, I. del Valle, J.A. de Saja, M.L. Rodríguez-Méndez. An electrochemical quartz crystal microbalance multisensor system based on phthalocyanine nanostructured films: Discrimination of musts. Sensors 15 (2015) 29233-29249.

doi: $10.3390 / s 151129233$

Impact Factor (2015 JCR): 2.033; Quartil (2015 JCR): Q1, Category: Instruments and Instrumentation. 
C. García-Hernández, C. García-Cabezón, C. Medina-Plaza, F. Martín-Pedrosa, Y. Blanco, J.A. de Saja, M.L. Rodríguez-Méndez. Electrochemical behavior of polypyrrol/AuNP composites deposited by different electrochemical methods: sensing properties towards catechol. Beilstein Journal of Nanotechnology 6 (2015) 2052-2061. doi: 10.3762/bjnano.6.209 Impact Factor (2015 JCR): 2.778; Quartil (2015 JCR): Q1, Category: Materials Science, Multidisciplinary; Physics, Applied.

C. Medina-Plaza, C. García-Hernández, J.A. de Saja, J.A. Fernández-Escudero, E. Barajas, G. Medrano, C. García-Cabezón, F. Martín-Pedrosa, M.L. Rodríguez-Méndez. The advantages of disposable screen-printed biosensors in a bioelectronic tongue for the analysis of grapes. LWT - Food Science and Technology 62 (2015) 940-947. doi: 10.1016/j.Iwt.2015.02.027 Impact Factor (2015 JCR): 2.711; Quartil (2015 JCR): Q1, Category: Food Science and Technology.

C. Medina-Plaza, C. García-Cabezón, C. García-Hernández, C. Bramorski, Y. BlancoVal, F. Martín-Pedrosa, T. Kawai, J.A. de Saja, M.L. Rodríguez-Méndez. Analysis of organic acids and phenols of interest in the wine industry using Langmuir-Blodgett films based on functionalized nanoparticles. Analytica Chimica Acta 853 (2015) 572-578. doi: 10.1016/j.aca.2014.10.046

Impact Factor (2015 JCR): 4.712; Quartil (2015 JCR): Q1, Category: Chemistry, Analytical.

M.L. Rodríguez-Méndez, C. Medina-Plaza, C. García-Hernández, J.A. de Saja, J.A. Fernández- Escudero, E. Barajas-Tola, G. Medrano. Analysis of grapes and wines using a voltammetric bioelectronic tongue. Correlation with the phenolic and sugar content. IEEE Sensors Journal IEEE Sensors 2014 Proceedings (2014) 2139-2142.

doi: 10.1109/ICSENS.2014.6985461

Impact Factor (2014 JCR): 1.762; Quartil (2014 JCR): Q2, Category: Engineering, Electrical and Electronic; Instruments and Instrumentation; Physics, Applied. 

Doctoral Thesis: Electronic tongue technology applied to the analysis of grapes and wines

Annex C: International research stay attestation letter 

The attestation letter from Prof. Wanekaya that certifies an international research stay is attached below:

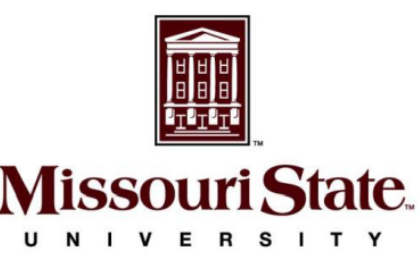

February 06, 2018

To whom it may concern

\section{RE: Celia Garcia Hernandez}

The purpose of this letter is to confirm that Celia Hernandez performed research in my lab at Missouri State University in Springfield, Missouri from August 2017 to December 2017. During that time she worked on a project entitled "In situ Formation, Stabilization and Activity of Protein Modified Gold Nanoparticles for Biological Applicatons" Lysozyme was used as a model protein to demonstate the in-situ reduction and stabilization of the gold nanoparticles prior to their modification by the layer-by-layer technique. The nanoconjugates were characterized by Dynamic Light Scattering Spectroscopy (particle sizing), zeta potential (surface charge analysis) and Ultraviolet-Visible Spectroscopy. Dynamic Light Scattering Spectroscopy confirmed that the sizes of the resulting nanoconjugates were below $100 \mathrm{~nm}$ after the layer-by-layer deposition procedures. Zeta potentials measurements also confirmed the altermation depostion of the oppositely charged layers. The enzymatic activity of the lysozyme was evaluated and confirmed using the bacteria Micrococcus lysodeikticus as a substrate. The success of this project has potential implications in the biomedical science field especially in protein delivery, drug delivery, nucleic acid delivery and biosensing applicatons.

Celia did all these work in less than 4 months including the completion of a draft manuscript. I am still reviewing the manuscript before I submit it for publication in a couple of weeks. In summary, Celia is a very brilliant and dedicated researcher. Her ability to design, execute, trouble shoot and optimize experiments is truly commendable. Her impressive experimental skills give me all reasons to believe that she will continue her remarkable efforts in the scientific field in future. If you have any questions regarding this letter, please do not hesitate to contact me.

Sincerely,

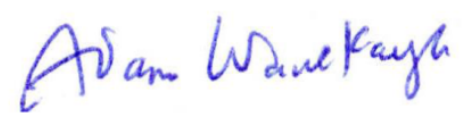

Adam K. Wanekaya, Ph. D

Professor of Chemistry

wanekaya@missouristate.edu

DEPARTMENT OF CHEMISTRY

901 South National Avenue, Springfield, MO 65897

Phone: (417) 836-5506 • Fax: (417) 836-5507

http://chemistry.missouristate.edu • chemistry@missouristate.edu 

Doctoral Thesis: Electronic tongue technology applied to the analysis of grapes and wines

\section{Annex D: Assessment reports}





\section{CSDUVa \\ Escuela de Doctorado Universidad de Valladolid}

\section{Universidad deValladolid}

Form 2T

ASSESSMENT REPORT OF A PhD THESIS PRIOR TO ITS DEFENSE

(As required by Section 2.1c. of the Regulation concerning doctoral thesis defense at UVa)

Full name: Carlos José Leopoldo Constantino

Department: Physics

University or Research Institution: University of São Paulo State (UNESP) - campus of Presidente Prudente

Regarding the thesis entitled: Electronic Tongue Technology Applied to the Analysis of Grapes and Wines.

Written by Mr./Mrs.: Celia García Hernández

Please, report your arguments and critical opinion on the following issues concerning the PhD thesis, writing as much as necessary:

1. Is the topic relevant? Are the research objectives well defined?

Yes, the topic is relevant. It is within the context of developing analytical methods to improve the food quality control.

Yes, the objectives are well defined. The main objective of this $\mathrm{PhD}$ thesis was to develop new electrochemical sensors with electrocatalytic materials to improve their performance (sensors) in terms of sensitivity. These sensors were implemented in electronic and bioelectronic tongues to study, mainly, antioxidants and polyphenols present in grapes and wines.

2. Is the selected methodology sound and suitable for the topic and the objectives pursued in the thesis?

Yes, the methodology is suitable. Different phthalocyanines, metal nanoparticles, carbon-based materials, and conducting polymers were applied in this $\mathrm{PhD}$ thesis to improve the sensitivity of the sensors, and enzymes were applied to improve the selectivity (specificity) of the sensors regarding the detection of phenols.

The developed sensors are based either on non-nanostructured or nanostructured materials, for which the nanotechnology played an important role in its preparation by using different surface modification techniques. In addition, some of the electrocatalytic materials were applied in a combined way to study the possible synergistic effect between them, targeting to improve the sensor responses.

The electronic and bioelectronic tongues were applied to analyze real wine and grape samples. Statistical analyses (multivariate data) were carried out to discriminate these samples and to establish correlations between the data obtained with the electrochemical sensors and the chemical parameters obtained by traditional analysis.

3. Is the body of reviewed literature up to date and complete? Have all relevant sources been considered and cited?

Yes, the review is appropriate and relevant sources were considered/quoted. For instance, the article I presented in this PhD thesis, entitled "Multisensor systems based on phthalocyanines for monitoring the quality of grapes", is a review that provides an overview of phthalocyanine-based electronic tongues for grape analysis, in which the use of non-nanostructured and nanostructured sensors and biosensors are considered. Besides, the chapter $\|$ of this $\mathrm{PhD}$ thesis is totally dedicated to literature review. This chapter II quoted 195 references. 


\section{CSDUVa \\ Escuela de Doctorado Universidad de Valladolid}

Universidad deValladolid

Form 2T

4. Does the thesis make original contributions that expand the current knowledge on the subject? Are these contributions relevant?

Yes, this $\mathrm{PhD}$ thesis brings original and relevant contributions to the field of sensor and biosensors. For instance, this $\mathrm{PhD}$ thesis is composed by 9 articles accepted for publication in international and important journals.

5. Is the thesis structure adequate to explain the research carried out and the results achieved? Is language used properly? Are formal elements, like figures or tables, well laid out and helpful to understand the research and results?

The answer is Yes for all the questions above. Considering the PhD thesis is a compilation of articles, the evaluation process of these articles by several referees helped with aspects such as explanation, language and formal elements.

6. Only if this is a compilation thesis (written in the format of a collection of articles): Is there a clear and coherent connection among the topics and methodology of the different articles that comprise the thesis? Do the introduction and conclusions of the thesis provide a unifying picture of the whole research?

Yes for all the questions above. The PhD thesis brings a resume with the main aspects related to each article, which makes easier to the reader the role comprehension of the PhD thesis.

7. Please mention three strengths and three weaknesses of this thesis.

Please, this $\mathrm{PhD}$ thesis is a compilation on 9 articles, published in international and important journals, as previous mentioned, between 2015 and 2019. Besides, the candidate Celia Garcia Hernandez is the $1^{\text {st }}$ author in 7 of these 9 articles, and the $2^{\text {nd }}$ author in the other 2 articles (one of them is the review, in which the advisor Maria Luz Rodriguez Mendez is the $1^{\text {st }}$ author). Therefore, I do not see weaknesses that deserve to be mentioned here.

8. If you think the thesis should NOT be defended in its current form, please mention the changes that you consider MUST be done before it can proceed to defense.

In my opinion, the PhD thesis can be defended in its present form.

9. Please mention other changes that MAY be done in order to improve the thesis quality, but that you do not consider strictly necessary to authorize its defense.

Nothing to declare.

10. Any other comments:

I would like to congratulate the candidate and the advisor for the quality of this $\mathrm{PhD}$ thesis, besides such productive period.

11. Please provide your recommendation to the Academic Board of the PhD Program:

$X \quad$ This thesis should be ADMITTED for defense, either in its current form or after taking into account the suggestions made in point 9 of this report. 


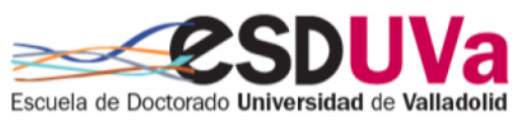

Universidad deValladolid

Form 2T

$\square \quad$ This thesis should be MODIFIED before its admission for defense in order to make the changes requested in point 8 of this report.

$\square$ This thesis should be REIECTED for defense, due to the arguments given in this report.

Place and date: Presidente Prudente, Brazil, September 30, 2019.

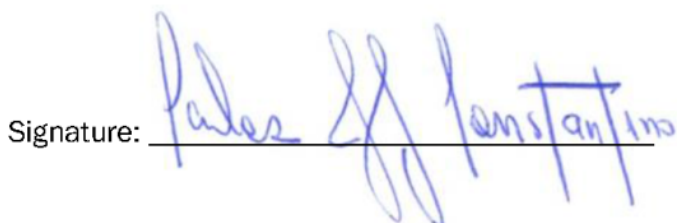

Notes: The length of this report is not restricted. Please remember to sign it (digital signatures are accepted). 



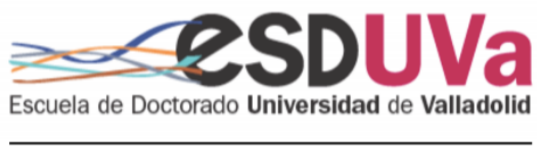

Universidad deValladolid

Form 2T

ASSESSMENT REPORT OF A PhD THESIS PRIOR TO ITS DEFENSE

(As required by Section 2.1c. of the Regulation concerning doctoral thesis defense at UVa)

Full name: LUÍS AVELINO GUIMARÃES DIAS

Department: Environment and Natural Resources

University or Research Institution: Polytechnic Institute of Bragança, Portugal

Regarding the thesis entitled: Electronic tongue technology applied to the analysis of grapes and wines

Written by Mrs.: Celia García Hernández

Please, report your arguments and critical opinion on the following issues concerning the PhD thesis, writing as much as necessary:

1. Is the topic relevant? Are the research objectives well defined?

The subject of the thesis is current and relevant, focusing on the construction of multi-sensor systems that allow the development of new analytical technical applications relevant to the research area of viticulture and oenology, with possible future applications in the wine industry.

The objectives of the research work are well defined, showing that the research work involved the construction of several electronic tongues, testing the response performance of working electrodes using various electrochemical materials, applying various electrochemical techniques and evaluating the responsiveness of the analytical systems built in the analysis of real samples, grapes, musts and wines.

2. Is the selected methodology sound and suitable for the topic and the objectives pursued in the thesis?

The doctoral work involved the application of electronic tongues in the analysis of grapes, musts and wines, being an added value building multi-sensor systems specific to these matrices, which would not happen if commercial systems were used. Therefore, the study of the application of different materials and construction techniques for electrode preparation in the electronic tongue's development made research work complex and demanding, as expected from a doctoral work, but very suitable to the topic since the work has the three steps referred in the work objectives: construction, performance evaluation and application.

3. Is the body of reviewed literature up to date and complete? Have all relevant sources been considered and cited?

Regarding the literature review (chapter 2), the organization of the text made by the author allows any reader to have a clear understanding of the importance of electronic tongues, referred to as a new sensory technology developed to evaluate the taste of food and beverages. It begins by referring the importance of the sensory panel in the food industry and its disadvantages, for which an electronic tongue can be an analytical solution. It makes a comparison with the gustatory system of the human tongue showing that the electronic tongue is an analytical tool that mimics it. Provides a background on early work with the electronic tongues, developments and applications. Later, presents the most applied principles of detection, the materials used in the construction of electrochemical sensors and techniques used in its construction, as well as the multivariate statistical methodologies for the data analysis. Finally, the paper presents examples of application in various scientific areas and commercial systems of electronic languages, focusing later on applications in the wine industry.

In the reviewed literature, the references are current and from relevant sources. There was no need to include any more particular references, so I think there is enough information for any reader to understand the contents explained. 


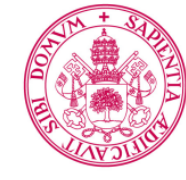

Universidad deValladolid

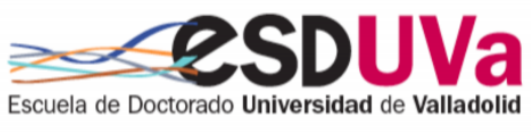

Form $2 \mathrm{~T}$

4. Does the thesis make original contributions that expand the current knowledge on the subject? Are these contributions relevant?

Yes, the relevance of the work is expressed in the 9 articles selected for this PhD thesis that were published, which are from journals with good scientific impact: 1) Journal of Porphyrins and Phthalocyanines; 2) Frontiers in Chemistry; 3) Food Research International; 4) Beilstein Journal of Nanotechnology; 5) Food Chemistry; 6) Beilstein Journal of Nanotechnology; 7) Sensors and Actuators; 8) Sensors; 9) Biomaterials Science. The journals are on food, sensors and materials scientific areas, showing that the doctoral research work allowed to expand the knowledge, in general, through the use of materials and recent techniques of electrodes construction, with applications in the analysis of grape, must and wine.

5. Is the thesis structure adequate to explain the research carried out and the results achieved? Is language used properly? Are formal elements, like figures or tables, well laid out and helpful to understand the research and results?

The doctoral dissertation has a suitable structure. It introduces the subject of electronic language allowing the reader to have a comprehensive understanding of how it works and the possible applications. In chapter 3, the initial introduction of the 9 selected articles allows an overview of the work done and the presentation of the motivations and objectives, as well as the summary of each research work allows to understand why the study was carried out under the PhD thesis.

The language present in the text is adequate, presenting the contents and results of the research work objectively, precisely and clearly.

The figures and tables presented are adequate and allowed to highlight explained concepts and schematize information. Globally, they allowed to summarize information that would be complicated to explain in the thesis text.

6. Only if this is a compilation thesis (written in the format of a collection of articles): Is there a clear and coherent connection among the topics and methodology of the different articles that comprise the thesis? Do the introduction and conclusions of the thesis provide a unifying picture of the whole research?

Yes, as I mentioned in the previous point to indicate that the structure of the thesis is adequate to explain the research work done. Globally, the different articles are based on electrodes construction using different materials and techniques and in their performance evaluation study as in electrode arrays, which can be applied in analysis of grape, must or wine samples. The electrode arrays were applied as an electronic tongue and, therefore, there is a connection between the topics and the methodology of the articles presented in the thesis.

The introduction, objectives and conclusions of the thesis are coherent with each other, showing that the thesis has a careful structure in order to allow the unification of the research work presented.

7. Please mention three strengths and three weaknesses of this thesis.

\section{Strengths:}

The work focused on building and testing several electronic tongues architectures that showed effective analytical potentialities in the analysis of vineyard products; The works show the potential application of voltammetric electronic languages in food analysis with advantages inherent in the methodology, such as the speed and simplicity of analysis; The electrodes built and tested in this work have great analytical application potential in other matrices, which should be considered as a major scientific contribution.

\section{Weaknesses:}

The weaknesses do not invalidate the research line followed in the thesis of developing, studying and applying sensor lines in the analysis of vineyard products. In fact, these weaknesses can be considered as future work objectives to develop based on the work presented in the thesis. Thus, it would be important to develop a single electronic tongue solution that enables applications across the wine production line by doing: a comparison of the analytical performance of the different electrodes in order to select them; developing methodologies and procedures that allow any 


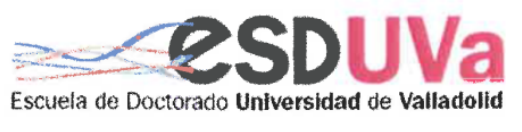

\section{Untvarsidad deValladold}

Form 2T

technician to use them, especially in in-situ analysis environments in the vineyard sector should be considered; including the development of a control platform to simplify the use of the analytical system and data treatment by any technician.

8. If you think the thesis should NOT be defended in its current form, please mention the changes that you consider MUST be done before it can proceed to defense.

9. Please mention other changes that MAY be done in order to improve the thesis quality, but that you do not consider strictly necessary to authorize its defense.

There are no corrections that MAY be done. The thesis presented has quality to be evaluated.

10. Any other comments:

No.

11. Please provide your recommendation to the Academic Board of the PhD Program:

Х This thesis should be ADMITTED for defense, either in its current form or after taking into account the suggestions made in point 9 of this report.

$\square \quad$ This thesis should be MODIFIED before its admission for defense in order to make the changes requested in point 8 of this report.

$\square$ This thesis should be REJECTED for defense, due to the arguments given in this report.

Portugal, October 4, 2019

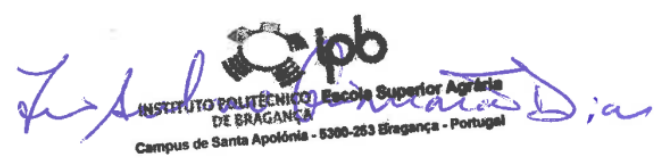


